\title{
Adrenocortical carcinoma
}

Citation for published version (APA):

Ettaieb, M. H. T. (2020). Adrenocortical carcinoma: an orphan disease with many faces. [Doctoral Thesis, Maastricht University]. Ridderprint. https://doi.org/10.26481/dis.20201218me

Document status and date:

Published: 01/01/2020

DOI:

10.26481/dis.20201218me

Document Version:

Publisher's PDF, also known as Version of record

\section{Please check the document version of this publication:}

- A submitted manuscript is the version of the article upon submission and before peer-review. There can be important differences between the submitted version and the official published version of record.

People interested in the research are advised to contact the author for the final version of the publication, or visit the DOI to the publisher's website.

- The final author version and the galley proof are versions of the publication after peer review.

- The final published version features the final layout of the paper including the volume, issue and page numbers.

Link to publication

\footnotetext{
General rights rights.

- You may freely distribute the URL identifying the publication in the public portal. please follow below link for the End User Agreement:

www.umlib.nl/taverne-license

Take down policy

If you believe that this document breaches copyright please contact us at:

repository@maastrichtuniversity.nl

providing details and we will investigate your claim.
}

Copyright and moral rights for the publications made accessible in the public portal are retained by the authors and/or other copyright owners and it is a condition of accessing publications that users recognise and abide by the legal requirements associated with these

- Users may download and print one copy of any publication from the public portal for the purpose of private study or research.

- You may not further distribute the material or use it for any profit-making activity or commercial gain

If the publication is distributed under the terms of Article $25 \mathrm{fa}$ of the Dutch Copyright Act, indicated by the "Taverne" license above, 

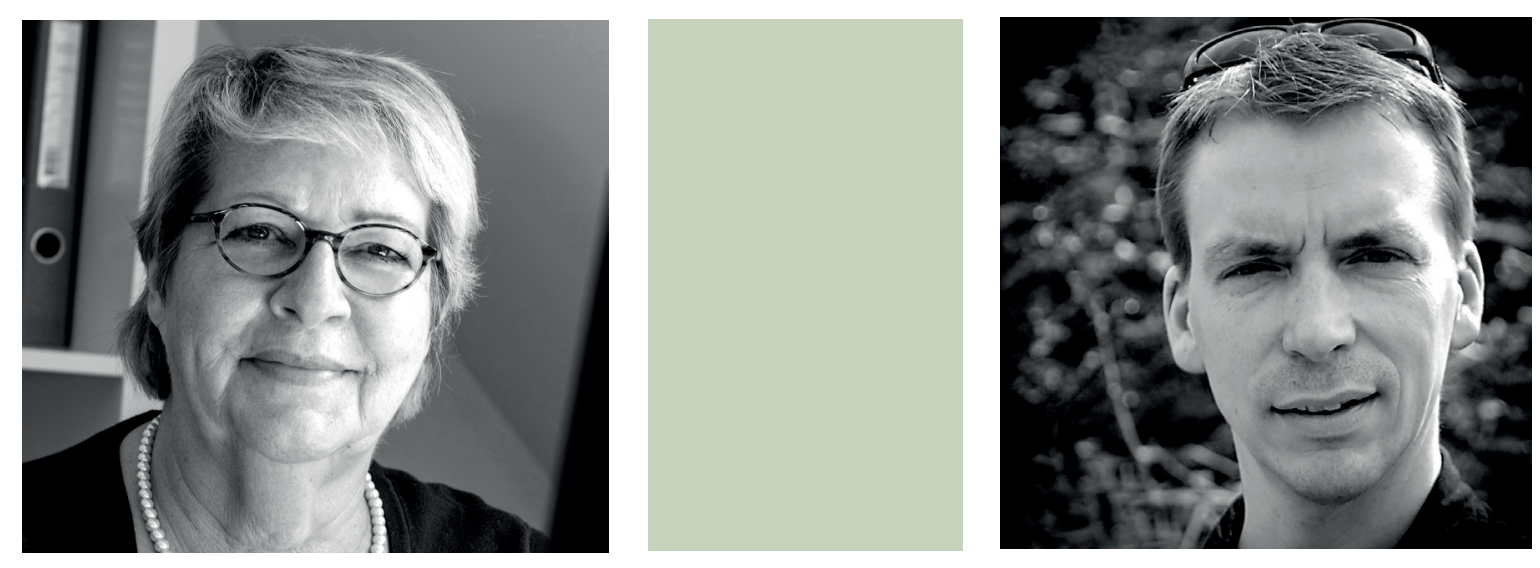

\section{Adrenocortical Carcinoma: an orphan disease with many faces}
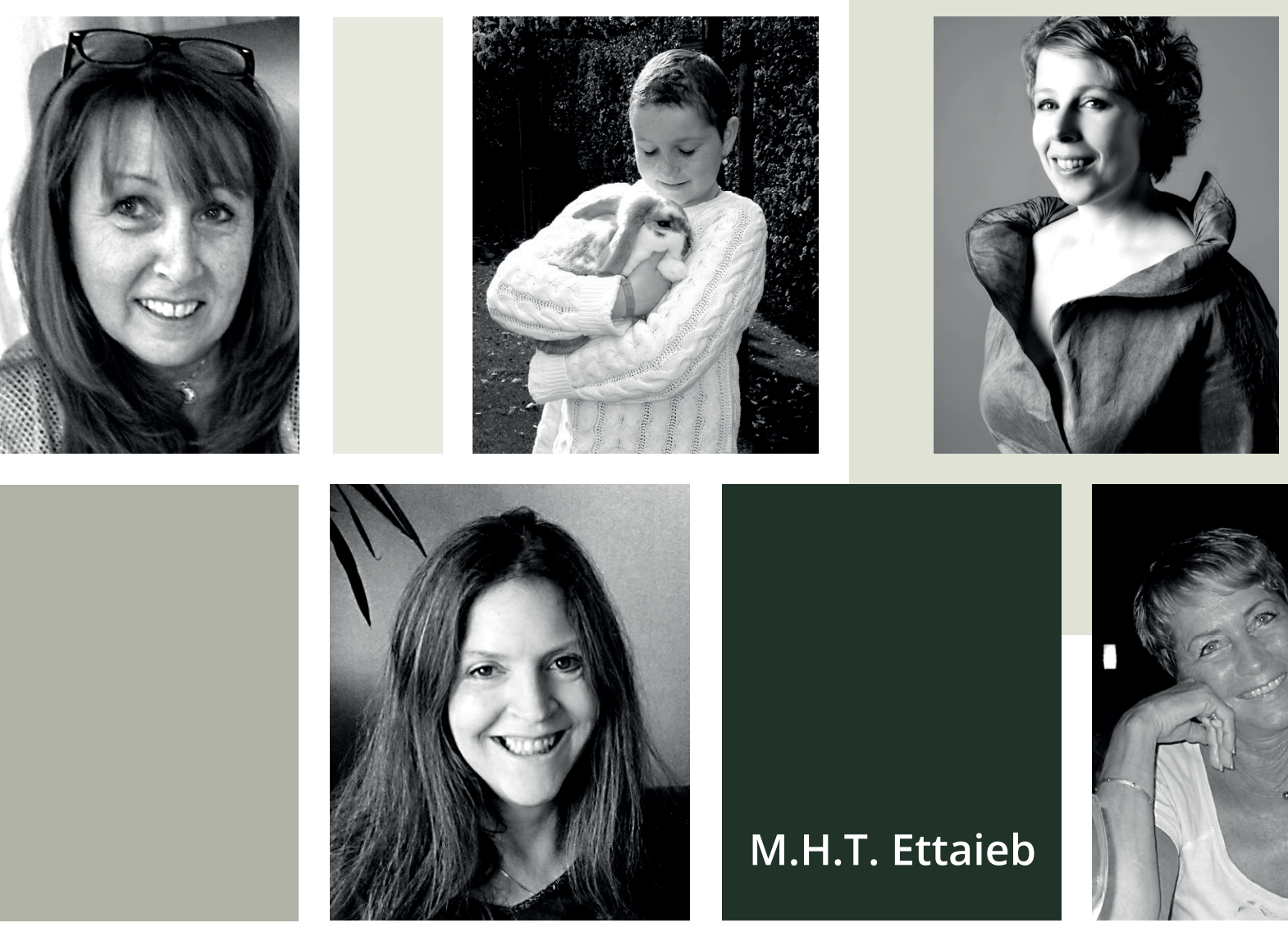

M.H.T. Ettaieb

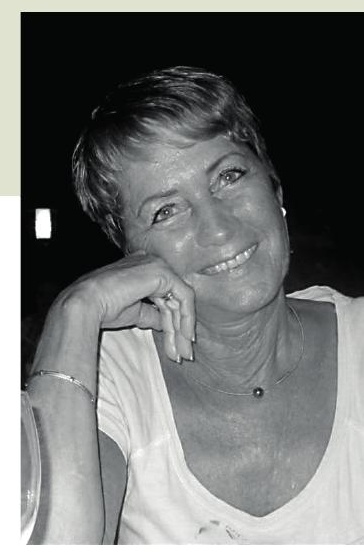





\section{Adrenocortical Carcinoma: an orphan disease with many faces}




\section{Colophon}

Copyright (c) 2020 MHT Ettaieb.

Printing: Ridderprint

Vormgeving: Publiss

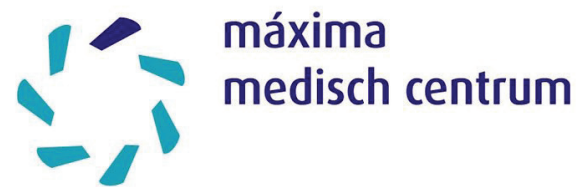




\title{
Adrenocortical Carcinoma: an orphan disease with many faces
}

\section{Bijnierschorscarcinoom:}

een zeldzame ziekte met vele gezichten

\author{
Proefschrift
}

Ter verkrijging van de graad van doctor aan de Universiteit Maastricht

Op gezag van de rector magnificus

Prof. Dr. Rianne M. Letschert

En volgens besluit van het College van Decanen

In het openbaar te verdedigen

Op Vrijdag 18 December 2020 om 12:00 uur

Door

Madeleine Hester Tonny Ettaieb

geboren te Naarden 
Promotor

Copromotor

Beoordelingscommissie Prof. Dr. V.C.G. Tjan-Heijnen (voorzitter)

Prof. Dr. N.D. Bouvy

Prof. Dr A.P. de Bruïne

Dr. M.R. Dahele

(Amsterdam Universitair Medische Centra, VUMC)

Prof. Dr. W.W. de Herder

(Erasmus Medisch Centrum Rotterdam) 
Aan mijn ouders,

Tonny Moos \& Ben Nejib Ettaieb 


\section{Table of Contents}

Chapter 1 General introduction 9

Part I Clinical prediction 38

Chapter 2 Synchronousversusmetachronousmetastasesinadrenocortical 41 carcinoma: an analysis of the Dutch adrenal network

Chapter 3 Development and internalvalidation of a multivariable prediction 63 model for adrenocortical-carcinoma-specific mortality

Part II Treatment strategies 84

Chapter 4 Population pharmacokinetic and pharmacogenetic analysis 87 of mitotane in adrenocortical carcinoma patients: towards individualized dosing

Chapter 5 Developing treatment for adrenocortical carcinoma 149

Chapter 6 Past, present and future of epigenetics in adrenocortical 177 carcinoma

$\begin{array}{ll}\text { Part III Elaboration } & 210\end{array}$

Chapter 7 General discussion and future perspectives 213

Chapter 8 Summary / Samenvatting (summary in Dutch) 233

Chapter 9 Impact Paragraph 243

$\begin{array}{ll}\text { Appendix } & 251\end{array}$

Curriculum vitae $\quad 252$

List of publications 253

Acknowledgments (in Dutch) / Dankwoord 255 



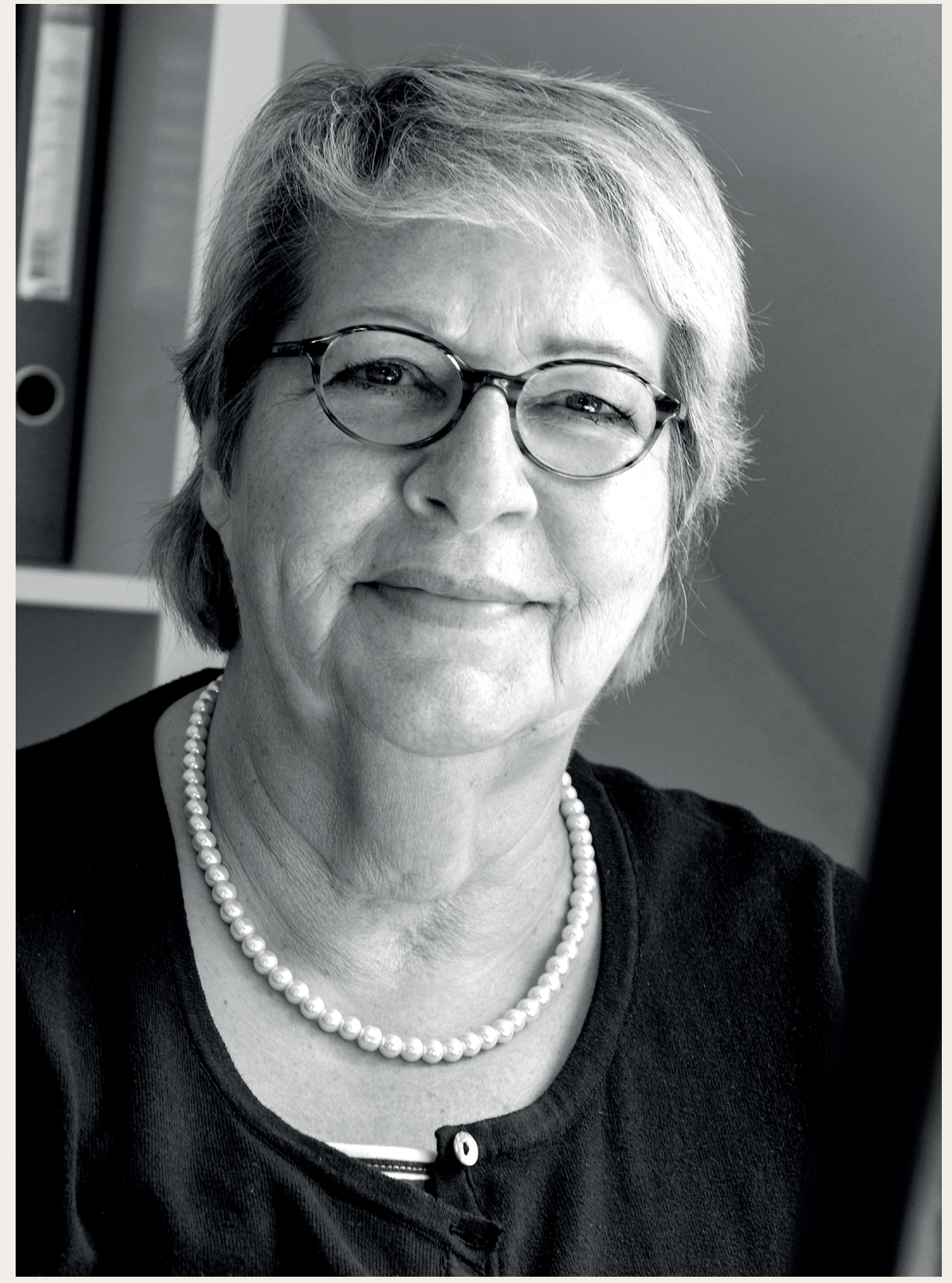


Chapter 01

General introduction 


\section{General introduction}

\section{Anatomy}

The adrenal glands are endocrine glands that produce a variety of hormones. The human body has two adrenal glands, there is one localized on the superior medial aspect of the upper pole of each kidney in the retroperitoneum.

The adrenal sizes vary in normal subjects, measuring about $4 \mathrm{~cm}$ long and $3 \mathrm{~cm}$ thick, and are surrounded by adipose tissue (1). A normal adrenal gland in an adult weighs approximately 4 to 6 grams. The left adrenal is larger and flatter than the right adrenal gland. The right adrenal gland is pyramid-shaped ("witch's hat") and lies above the upper pole of the right kidney, between the liver and the diaphragm. The left adrenal gland is semilunar-shaped and is found between the kidney and aorta, near the tail of the pancreas and the splenic artery.

Each adrenal gland holds two functionally distinct endocrine units that are developmentally, structurally and functionally different. The outer part is called the adrenal cortex, composing 80 to 90 percent of the volume of a normal gland, and the inner part the adrenal medulla, constituting 10 to 20 percent of the volume of a normal gland. The cortex develops from mesoderm whereas the medulla develops from the neural crest.

The adrenal cortex is divided into three distinct functional zones producing steroids:

- An outer zone, 15\% of the cortex, called zona glomerulosa secreting mineralocorticoids (aldosterone), which regulate sodium and potassium homeostasis.

- A middle zone, 75\% of the cortex, called zona fasciculata secreting glucocorticoids (most importantly, cortisol)

- An inner zone, $10 \%$ of the cortex, called zona reticularis secreting sex steroids (primarily androgens).

The adrenal medulla consists of so called chromaffin cells, because they stain brown with chromium salts, secreting catecholamines: epinephrine and norepinephrine, which regulate many cardiovascular and metabolic processes (2). 


\section{Adrenal gland tumors}

Adrenal tumors (tumor is Latin for swelling) or masses of the adrenal gland are common. Adrenal tumors include benign and malignant adrenocortical tumors and benign and malignant tumors of the adrenal medulla named pheochromocytomas (PCC).

Adrenal masses are often referred to as adrenal incidentaloma since they are serendipitously discovered by radiologic examination. Incidentalomas are defined as adrenal masses of one centimeter or more in diameter (3). They are found in up to $10 \%$ of the imaging done for other reasons, being more frequent in older patients. In autopsy studies, the prevalence of incidentalomas is two percent, ranging from one to nine percent. The prevalence is higher in obese, diabetic, and hypertensive patients. $(4,5)$

\section{Adrenocortical carcinoma}

Adrenocortical carcinoma (ACC) is a rare aggressive neoplasm with poor prognosis. ACC has an annual incidence of 0.5 -2.0 patient per million people per year (6-9). ACC accounts for $0.02 \%$ to $0.2 \%$ of adult cancers and about $0.2 \%$ of all cancer deaths in the United State (7,10-12). ACC has a bimodal age distribution with a peak $<5$ years of age and a second peak during the fourth to fifth decades of adult life, but the tumor can appear at any age. One point three percent of all childhood cancers are ACCs, suggesting a higher relative incidence early in life. Interestingly, the incidence of pediatric ACC is 10-15 times higher in children in southern Brazil, which is related to an inherited germline p53 mutation(13,14).

Adrenocortical carcinoma is slightly more common in women than men with a ratio of 1.5:1 (15). Studies show that the left adrenal is more frequently affected. 


\section{Pathogenesis and genetics}

ACC pathogenesis is heterogeneous and still incompletely understood. Most of the progress in understanding its pathogenesis and identifying genes involved in ACC comes from the study of (rare) familial diseases. Although most cases of ACC are sporadic with no identifiable risk factor it can be a part of hereditary tumor syndromes such as:

- Multiple endocrine neoplasia type 1 (MEN1): mutations in the MENIN gene on chromosome 11q13. Approximately 10\% of MEN1 patients have distinct adrenal tumors, and of these, up to $14 \%$ are malignant $(12,16)$.

- Li-Fraumeni syndrome (LFS): Mutation of tumor suppressor protein TP53, located on 17p13.Three to 10\% of LFS associated cancers are ACCs. (17)

- Lynch syndrome: mutations in genes involved in DNA mismatch repair genes MSH2, MSH6, PMS2, MLH1, and TACSD1/EPCAM. The prevalence of Lynch syndrome in patients with ACC has been described approximately 3 (18).

- Beckwith-Wiedeman syndrome (BWS): alterations of DNA methylation of the 11 p15 locus, which harbors the coding regions for Insulin-growth-factor 2(IGF2), the cell cycle regulator CDKN1C, and the non-translated RNA, H19 (19). Up to $15 \%$ of all malignancies in BWS are ACC $(12,20)$.

- Familial adenomatous polyposis coli (FAP): inactivating germline mutation of the tumor suppressor gene adenomatous polyposis coli (APC) gene, which encodes for a downstream regulator of the Wnt/ $\beta$-catenin pathway (21). So far, six cases of ACC have been described in FAP patients (22).

- Werner syndrome: a premature aging disease caused by the mutation in the WRN gene (23).

- Neurofibromatosis type 1 (NF1): mutation in the NF1 gene that encodes neurofibromin. ACC has been reported in four patients with neurofibromatosis type 1 (NF1) (22).

The familial cancer susceptibility syndromes described above established that there are three major dysfunctional molecular pathways in ACC: the IGF pathway, the Wnt pathway and TP53 (24).

\section{IGF pathway}

The IGF pathway is involved in the development and in the maintenance of adrenocortical functions. The pathway consists of ligands (IGF-1 and IGF-2), receptors, 
binding proteins and binding protein proteases. The main role of IGF-2 lies in fetal development and growth, whereas IGF-1 mainly acts postnatal.

Chromosome locus 11p15, contains the genes cyclin-dependent kinase inhibitor (CDKN1C), IGF2 and H19, structurally organized in a cluster. Normally, these genes are expressed monoallelically, in a parent-of-origin-specific manner. IGF2 is maternally imprinted, therefore only the paternal allele is expressed. The paternal alleles of CDKN1C and H19 are silenced by imprinting, thus only the maternal alleles are expressed $(24,25)$. CDKN1 acts as a negative regulator of cell proliferation, H19 however acts as a transcriptional repressor of IGF2.

IGF2 is highly overexpressed in majority of ACCS, whereas the expression H19 and/ or CDKN1C is decreased. Overexpression of IGF2 is a result of loss of the maternal allele - loss of heterozygosity $(\mathrm{LOH})$ - and duplication of the paternal allele, called paternal isodisomy. Also, by loss of imprinting (LOI) because of demethylation of the maternal allele $(26,27)$. IGF2 regulates the growth and apoptosis of cells, and interact with insulin-like growth factor 1 receptor (IGF1R). The latter is also found to be overexpressed in ACC. Activation of IGF-1R results in stimulation of downstream signaling pathways including the mitogen-activated protein kinase (MAPK) and phosphoinositol3-kinase (PI3-AKT) pathway, leading to increased cell proliferation and survival $(28,29)$.

IGF2 overexpression by LOH is associated with poorer outcome (30).

\section{Wht pathway}

The Wnt/B-catenin pathway is active during normal embryonic development and has been shown to play an important role in organ development. Dysregulation of Wnt signaling has been found in a variety of cancers, but it was first identified and intensively researched in FAP patients. Research has shown that is also plays and important role in adrenocortical tumorigenesis. It are the activating mutations of the Catenin Beta 1 gene (CTNNB1), and deletions in the Zinc And Ring Finger 3 (ZNFR3) gene that leads to activation of the Wnt/ $\beta$-catenin pathway in ACC. Consequently this activation of the Wnt pathway induces cell proliferation and resistance to apoptosis.

Furthermore, the somatic mutation of the CTNNB1 is an independent predictor of less favorable survival in ACC. (31)

TP53

Germline inactivating TP53 mutations are very frequent in pediatric ACCs, but rare in adult patients with ACC, explaining the relative increase in incidence in childhood. 
The prevalence of germline TP53 mutations in children diagnosed with ACC ranges from 50-97\%. Somatic TP53 mutations contrary are common in adult patients, being described in $25-70 \%$ of samples (32).

TP53, known as the 'guardian of the genome', is located on chromosome 17p13 and its main functions are halting the cell cycle and/or inducing apoptosis in response to DNA damage. Unable to do so when mutated propagation of a corrupted genome can result in malignancy (33).

\section{Epigenetics}

Genetic studies aimed at adrenal tumors have identified multiple molecular alterations as factors in ACC carcinogenesis. Increasing evidence suggests that DNA methylation, the addition of methyl group to the cytosine pyrimidine ring or adenine purine ring of the DNA molecule without changing the DNA sequence, in addition to genetic modification causes altered patterns of gene expression resulting in tumorigenesis. In normal cells, DNA methylation is responsible for selective regulation of gene expression. The process of DNA methylation may occur throughout the genome in $C$ phosphate-G (CpG) dinucleotides. The genome overall has a low CpG content, except for areas in gene promoter regions. These $\mathrm{CpG}$ dinucleotides clusters in gene promoter regions are called CpG islands. Methylation of CpG islands in a gene promoter region mechanically blocks transcription by creating a barrier. An increased prevalence of CpG island methylation has been proposed to be involved in carcinogenesis of several tumors and has also been identified in adrenocortical adenoma and carcinoma (24).

\section{Genetic screening}

Currently there is no genetic screening for ACC where most ACCS are sporadic but it is important to consider the occurrence of ACC as part of a syndrome. Actually, Chompret criteria advocated for TP53 genetic testing for all patients diagnosed with ACC regardless of age at diagnosis or family history (34).

Aside from genetic predisposition, no risk factors have been confirmed. Smoking in men and contraceptive use in women, have been suggested as risk factors for ACC (35). 


\section{Clinical presentation}

Early diagnosis of ACC is very challenging because of the rarity of the disease and the lack of distinct and specific alarm symptoms. Most physicians are unlikely to encounter the disease and as a result are unfamiliar with diagnosing and treating ACC.

Often patients present with nonspecific complaints that are related to the tumor mass effect or endocrine disturbances.

Adrenocortical carcinoma can grow up to more than 20 centimeters. The increase in mass can cause abdominal or flank pain or other abdominal discomfort e.g. nausea, vomiting or fullness $(6,36,37)$. Thirty percent of patients present with abdominal mass effects.

Most patients, $40 \%$ to $60 \%$, present with symptoms of steroid hormone excess (glucocorticoids, mineralocorticoids, androgens).

When there is an excess of hormone secretion, ACC is referred to as functional. Depending on which hormones are over-secreted, different clinical consequences manifest: Cushing syndrome, virilization syndrome, feminization syndrome or a mixed Cushing-virilization syndrome. ACC is nonfunctional when the tumor does not secrete excessive hormones or in insufficient quantities to have clinical consequences.

In these 'functioning' ACCs, hypercortisolism, or Cushing syndrome, is the most common presentation of hormone excess (50\% to $80 \%$ ) with patients showing profound muscle atrophy, severe hypertension and diabetes mellitus (12). Women can present with signs and symptoms of androgen secreting ACC - acne, hirsutism and virilization (deepening of the voice, male pattern baldness and oligoamenorrhea) while men can present with estrogen-secreting ACC leading to feminization gynecomastia, loss of libido and testicular atrophy. It should be noted that androgensecreting tumors in men and estrogen secreting tumors in women, may not result in clinically significant syndromes (10).

Aldosterone-producing ACC is rare (Conn syndrome), symptoms are hypertension with hypokalemia. However, severe hypokalemia is also caused by elevated cortisol secretion.

Nonspecific symptoms of malignancy, such as fever, weight loss or general malaise affect only a small minority of ACC patients.

\section{Diagnosis}

The ACC working group of the European Network for the Study of Adrenal Tumors (ENSAT) has proposed standards for the diagnostic procedures (37). It is of most 
importance that each step of the management strategy should be decided in the setting of a multidisciplinary team including different expertise (endocrinology, radiology, pathology, surgery, oncology), in expert centers.

Adrenal biopsy is discommended, because of increased risk of (needle-track) metastases, in the diagnostic work-up of patients with suspected ACC. Unless, there is evidence of metastatic disease that interferes with surgery and histopathologic diagnosis is required to optimize oncological management.

\section{Imaging}

There are currently three main imaging techniques available for the differentiation of malignant and benign adrenal tumors: Computed tomography (CT), magnetic resonance imaging (MRI) and positron emission tomography (PET) with 18F-2-deoxyd-glucose (FDG) (mostly combined with CT; FDG-PET/CT). Endoscopic ultrasonography (EUS) has no major role in the diagnostic procedures of ACC (38). These imaging methods cannot determine the exact identity of the adrenal mass but (imaging) criteria have been defined to differentiate between benign and malignant. Adrenocortical carcinomas are mostly inhomogeneous and irregular. Tumor size is considered to be an important marker for malignancy. Masses of more than $6 \mathrm{~cm}$ in diameter are considered malignant until proven otherwise (15). Local invasion or tumor extending in to the inferior vena cava, as well as lymph node or other metastases are signs of malignancy and often found in advanced ACC.

Hounsfield units ( $\mathrm{HU}$ ) measured with non-contrast $\mathrm{CT}$ provides additional information in the diagnosis of adrenal tumors. Hounsfield unit is a relative quantitative measurement of radio density used by radiologists in the interpretation of CT images. Most benign adenomas are lipid-rich and have low HU whereas ACC are characterized by low fat content and hence higher HU density. A threshold value of $10 \mathrm{HU}$ is used to distinguish adrenal masses, where a low density/ attenuation value $\leq 10 \mathrm{HU}$ is considered as benign. In fact, the ACC working group of ENSAT states that only non-contrast CT is sufficiently reliable to rule out ACC when the adrenal lesion is homogenous and has low CT density $\leq 10 \mathrm{HU}$ (37). Additional delayed contrast-enhanced CT is used, analyzing washout of contrast medium, especially to discriminate between lipid-poor adenoma and ACC. A wash out $<50 \%$ and a delayed attenuation of $>35 \mathrm{HU}$ (on 10-15 minutes delayed contrast CT) are suspicious for malignancy $(6,39-41)$.

Magnetic resonance imaging lesions with the dynamic gadolinium enhancedand chemical shift technique is similar effective in characterizing adrenal lesions. Adrenocortical carcinomas are isointense to the liver on T1-weighted images but are inhomogeneous and show an increase intensity in T2-weighted sequences. When 
using gadolinium, adenomas show mild enhancement with quick washout, whereas malignant tumors show strong enhancement and slower washout. MRI is considered to be superior to CT in assessing the extent of vascular invasion, especially into the inferior vena cava.

With FDG-PET, a high uptake of FDG demonstrates increased glucose metabolism and indicates malignancy, but the FDG-PET cannot differentiate reliably ACC from a metastasis from other tumor or even a pheochromocytoma (42). PET imaging has been proposed as possibly the best second line test to assess indeterminate masses by unenhanced CT (37).

Imaging from chest to pelvic is done to evaluate for metastases. It is mandatory to systematically and rapidly evaluate for metastases, before initiation of any anti-tumor treatment. Thoraco-abdomino-pelvic imaging will cover the vast majority of metastatic locations, which most often are lung and liver (37).

\section{Hormonal work up}

Hormonal analyses is crucial because of various reasons: 1) to assess if there is hormone excess 2) to establish the adrenocortical origin of a tumor (pheochromocytoma has to be excluded prior to an invasive procedure where it requires a specific perioperative management) 3) to suggest malignancy (androgen or estrogen production) 4) to establish suitable tumor markers for follow-up and surveillance and 5) to optimize the perioperative management (e.g. glucocorticoid replacement therapy). A comprehensive work-up as advised by ENSAT is presented in the table below $(15,37,43)$. 
Glucocorticoid excess (Minimum three out of four tests)

Dexamethasone suppression test: $1 \mathrm{mg}$

Excretion of free urinary cortisol (24h urine)

Late-night salivary cortisol (23:00)

Basal adrenocorticotropic hormone (ACTH)

Sexual steroids and steroid precursors

Dehydroepiandrosterone sulphate (DHEA-S)

17-OH-progesterone (serum)

Androstenedione (serum)

Testosterone (serum, only in woman)

17-beta-estradiol (serum, only in men and postmenopausal women)

11-Deoxycortisol

Mineralocorticoid excess

Potassium (serum)

Aldosterone/renin ratio (only in patients with arterial hypertension and/or hypokalemia)

Exclusion of pheochromocytoma

Meta- and Normetanephrines (plasma and urine)

\section{Urinary steroid profile}

Urine steroid metabolomics (in $24 \mathrm{~h}$ urine samples) are proofing to be a significant alternative diagnostic tool for discriminating between benign and malignant adrenal lesions. Tetrahydro-11-deoxycortisol (THS) levels have been shown to be able discriminate between ACC and benign adrenal mass with high specificity (44-48).

\section{Pathology}

Adrenocortical carcinoma diagnosis should be confirmed by histopathology, preferably by and experienced pathologist. Different diagnostic scores have been introduced for the diagnosis of carcinoma versus adenoma. The Van Slooten Index (VSI) is a weighted system based on seven histopathological parameters(regressive changes, loss of normal structure, nuclear atypia, nuclear hyperchromasia, abnormal nucleoli, mitotic activity, capsular and/or vascular invasion), each combined with a numerical value. A score of eight or higher corresponds to a high probability of malignancy(49). The Weiss score system is recommended and most used (50). This classification is based on a combination of nine histologic criteria. The nuclear grade 
uses the criteria of Fuhrman et al(51): grade 1) round nuclei, homogenous, small size, no nucleoli; grade 2) nuclei slightly irregular, more voluminous, conspicuous nucleoli at $\times 400$; grade 3 ) irregular nuclei, voluminous nucleoli at x100; grade 4 idem grade 3 with monstrous cells with very irregular nuclei.

The presence of three or more Weiss criteria highly correlates with malignancy, whereas scores between zero and two defines the adrenal adenoma (52). A score of two or three may be considered borderline malignant (tumors with a score of three and an benign clinical course have been noted) and should be treated cautiously where they have uncertain malignant potential. The guideline advices to use additional classification systems and/or the addition of reticulin stain assessment $((37,53)$.

\begin{tabular}{lll}
\hline The Weiss system & \multicolumn{2}{l}{ Weight of criteria } \\
\hline Histological criteria & 0 & 1 \\
& 1 and 2 & 3 and 4 \\
Nuclear grade & $\leq 5$ for 50 fields & $\geq 6$ for 50 fields $\times 400$ \\
Mitotic rate & $\times 400$ & \\
& No & Yes \\
Atypical mitotic figures & $>25 \%$ & $\leq 25 \%$ \\
Eosinophilic tumor cell cytoplasm/Clear cells & $\leq 33 \%$ surface & $>33 \%$ surface \\
Diffuse architecture & No & Yes \\
Necrosis & No & Yes \\
Invasion of venous structures & No & Yes \\
Invasion of sinusoidal structures & No & Yes \\
Capsular invasion & & \\
\hline
\end{tabular}

\section{Immunohistochemistry}

There are two important immunohistochemistry criteria that can help differentiate carcinomas from adenomas: The steroidogenic factor 1 (SF-1), a transcription factor expressed primarily in the hypothalamus, pituitary, and steroidogenic organs like adrenal glands, testes, and ovaries. Besides discriminating between adenoma and carcinoma it also has been suggested as prognostic factor for ACC (54).

The cellular proliferation marker, Ki-67, is a protein involved in different phases of the cell cycle. It is a key marker associated with proliferating cancer cells and a poor prognosis $(55,56)$. 


\section{Upcoming}

Of upcoming interest is a so called liquid biopsy, which is a minimal invasive test using a sample of blood. This can be used to look for cancer cells from a tumor that are circulating in the blood or for pieces of DNA from tumor cells that are in the blood. Circulating tumor cells (CTC) have been found in the blood of ACC patients. It was shown to support differential diagnosis between benign and malignant adrenocortical tumors but it needs to be confirmed in a larger study cohort (57).

Circulating cell-free DNA (cfDNA) describes DNA that is freely circulating in the bloodstream, but is not necessarily of tumor origin. It was found that in cancer patients increased amounts of cfDNA circulates which is caused by additional circulating tumor DNA (ctDNA). In a pilot study cfDNA was found in ACC patients but there were difficulties identifying ctDNA (58). Another study was able to find ctDNA only in a subset of ACC patients (59).

\section{Staging}

In 2004, the International Union Against Cancer (UICC) and the World Health Organization published the first staging classification based on the Tumor, Node, Metastasis (TNM) criteria for ACC. In 2008, an analysis based on the German ACC Registry revealed several shortcomings with this classification. Therefore, the European Network for the Study of Adrenal Tumors (ENSAT) developed a revised staging system(60). This staging system defines stage I (T1 NOMO) and stage II (T2NOMO) as strictly localized tumors with a size of $\leq 5 \mathrm{~cm}$ or $>5 \mathrm{~cm}$, respectively. Stage III tumors are characterized by infiltration of surrounding tissue, positive regional lymph nodes or a tumor thrombus in the renal vein and/or vena cava (T1-2N1M0 or T3-4NO-1M0). Stage IV is defined by the presence of distant metastasis (T1-4NO-1M1).

In 2015 Libe et al. suggested a modified ENSAT (mENSAT) staging system. It defined stage III as T3-4NOMO and stage IVa, IVb, IVc according to the number of tumorinvolved organs (including the primary tumor and the ' $\mathrm{N}$ ' as 'organ'): 2, 3 or $>3$, respectively (61). It has not been implanted in European Society of Endocrinology Clinical Practice Guidelines yet.

\section{Treatment}

Despite a lot of effort to improve care for patients with ACC, treatment still has limited opportunities.

The only potentially curative treatment for localized ACC is a radical surgical resection (R0). Open adrenalectomy by an experienced surgeon is recommended. There is 
growing support that for tumors without any evidence of local invasion laparoscopic adrenalectomy could be considered if the surgeon has sufficient experience in these types of surgery and respects the principles of oncologic surgery $(37,62,63)$.

Even after complete resection, recurrence rates are high with reported percentages of up to $91 \%(64,65)$. Recurrence free survival is worse after a surgery with microscopically positive margins (R1) or macroscopically positive margins (R2) (66), stressing the importance of a carefully planned and executed initial surgical treatment. Therefore the guidelines suggest that if the first surgery was suboptimal and R2, to discuss repeat surgery in a multidisciplinary expert team (37).

Surgery is also important in case of recurrence $(67,68)$. For selected patients a metastasectomy has shown beneficial (69-71). In the setting of metastatic disease at primary presentation, the role of aggressive surgery is considered an individualized decision.

\section{Mitotane}

Currently, the only FDA approved adjuvant treatment of ACC is the adrenocorticolitic agent mitotane. Mitotane or o,p'-DDD (1-(o-chlorophenyl)-1-(p-chlorophenyl)-2,2dichloroethane), is an isomer of Dichlorodiphenyldichloroethane (DDD), an analogue of the insecticide dichlorodiphenyltrichloroethane (DDT).

In 1948 Mitotane* was shown to produce adrenal atrophy in dogs by inhibiting $11 \beta$-hydroxylation and cholesterol chain cleavage in the mitochondria of steroidogenic cells therefore blocking cortisol synthesis $(72,73)$. These observations led to the discovery of the potential value of mitotane in the treatment of adrenal cortical carcinoma and in fact it has been used for treating advanced ACC since the sixties(74). Although the antitumor activity of mitotane is well-recognized, the exact mechanism for its cytotoxic activity is poorly understood. In addition to disruption of mitochondria (75,76), endoplasmatic reticulum stress was identified as a key molecular pathway activated by mitotane with sterol-O-acyl-transferase 1(SOAT1) as key molecular target. Mitotane inhibits SOAT1 which triggers lipid-mediated endoplasmic reticulum stress and apoptosis in adrenocortical carcinoma cells (77).

Mitotane is difficult to manage and treatment is unsatisfactory at multiple levels. Mitotane is commercially available only as tablets of $500 \mathrm{mg}$ under the brand name Lysodren $\circledast$. Intestinal absorption of mitotane varies significantly and is best when taken with fatty substances (e.g. chocolate, milk) (78). When absorbed mitotane is distributed in the body and metabolized in the liver, $25 \%$ is recovered in the urine. Because mitotane is lipophilic, the drug reaches highest concentrations in adipose tissue but can also be detected in the brain, adrenals, liver, bile and serum (79). 
A close correlation of mitotane plasma levels with both efficacy and adverse effects are known, therefore plasma concentrations between 14 and $20 \mathrm{mg} / \mathrm{L}$ are aimed at $(80,81)$. Reaching and maintaining these therapeutic levels is complicated. Mitotane has a low bio-availability, about 35\%-40\% of the drug is absorbed from the gastrointestinal tract and stored in adipose tissue (82). The half-life of mitotane is extensive, elimination ranges from 18 to 159 days with a median of 53 days $(78,83)$. The large distribution volume and long half-life of mitotane impede the ability to predict mitotane plasma. Furthermore, there is a high inter-individual variability in the pharmacokinetics (PK) of mitotane (84). Currently, the dosage tapering is highly expert-based. It may take up to several months to reach target plasma levels, months in which the cancer is unrestrained.

Two dosage regimens have been suggested to reach therapeutic levels. A low dose regimen which builds up in 14 days to 3 grams a day and a high dose regimen which builds up to 6 grams a day (85). The daily dosage is often divided into two or three doses, because i.e. 6 grams of mitotane is 12 tablets Lysodren ${ }^{\circledR}$. The dose is adjusted until it reaches an 'optimal' dose that gives the best results, mitotane levels predict treatment response, without causing unacceptable side effects, frequently monitoring plasma trough levels (at least 12 hours after the last dosage) accordingly $(80,86)$.

Side effects are dose-dependent: plasma levels above 20mg/l may be associated with severe side effects and bring no further therapeutic advantage. However, if a patient tolerates high mitotane levels it can be considered to continue treatment. Gastrointestinal disturbances occur in about 80\% of the patients and consist of anorexia, nausea or vomiting, and in some cases diarrhea. It has been reported that gastrointestinal symptoms are particularly evident at the beginning of treatment with a threshold of $5 \mathrm{mg} / \mathrm{l}$, and relapse at any increment in mitotane dose $(87,88)$.

The central nervous system is affected in $40 \%$ of the patients with a reported threshold of $15 \mathrm{mg} / \mathrm{l}$. These effects consist primarily of depression as manifested by lethargy and somnolence (25\%) and dizziness or vertigo (15\%). At high doses and after prolonged utilization, brain function impairment can occur which is reversible after treatment is terminated. Skin toxicity has been observed in about 15\% of patients. In women ovarian cysts may develop and gynecomastia in men. Moreover, mitotane exerts a complex effect on the endocrine system that may require multiple hormone replacement therapy in which glucocorticoid replacement is obligatory (87-89).

Mitotane is given in adjuvant setting in those patients without macroscopic residual tumor after surgery with a perceived high risk of recurrence. Adjuvant treatment is given for at least 2 years $(37,65,90)$. Mitotane is also given for recurrent disease and advanced disease possibly in combination with intravenous chemotherapy.

* In 1948 these first observations in eleven dogs were done with technical grade DDD. Nichols and Hennigar [195] (92) and Cueto and Brown [1958] (93) worked with technical DDD and its fractions and identified o,p'DDD (mitotane) as the active fraction (88). 
Overall, mitotane leads to stable disease or partial remissions in 30\% of cases $(12,91)$.

\section{Chemotherapy}

Several cytotoxic agents have been studied in ACC. The FIRM-ACT trial was the first ever conducted randomized controlled trial in ACC that confirmed the efficacy of chemotherapy and the superiority of etoposide, doxorubicin, cisplatin, mitotane (EDP-M) over streptozotocin, mitotane. Patients in the EDP-M group had a significantly higher response rate than those in the streptozotocin-mitotane group and longer median progression-free survival (5.0 months vs. 2.1 months) (94). EDP-M is the first therapy of choice in metastasized disease, mitotane mono therapy can be considered with less aggressive slow growing tumors.

Figure 1 shows different drugs that are currently used or previously studied in ACC with their corresponding cellular targets and pathways involved. Figure 2 shows response rates from studies with systemic therapies in ACC. Unfortunately, the advanced molecular understanding of ACC has not yielded a major therapeutic breakthrough yet and hope remains to identify better treatments compared to the currently used options.

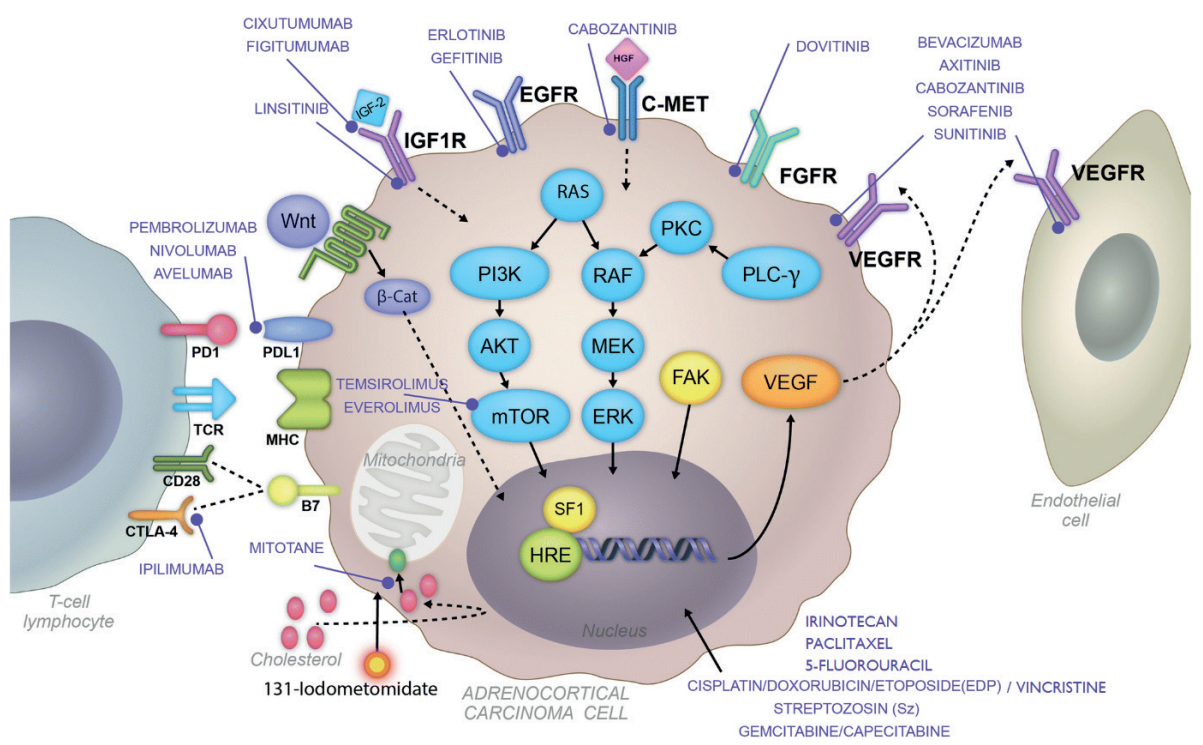

Figure 1 | Variable pathways and therapeutic targets in ACC and drugs studied or currently used. Adapted with permission from Springer Nature (c) Jasim and Habra, Current Oncology Reports 2019 (95) 


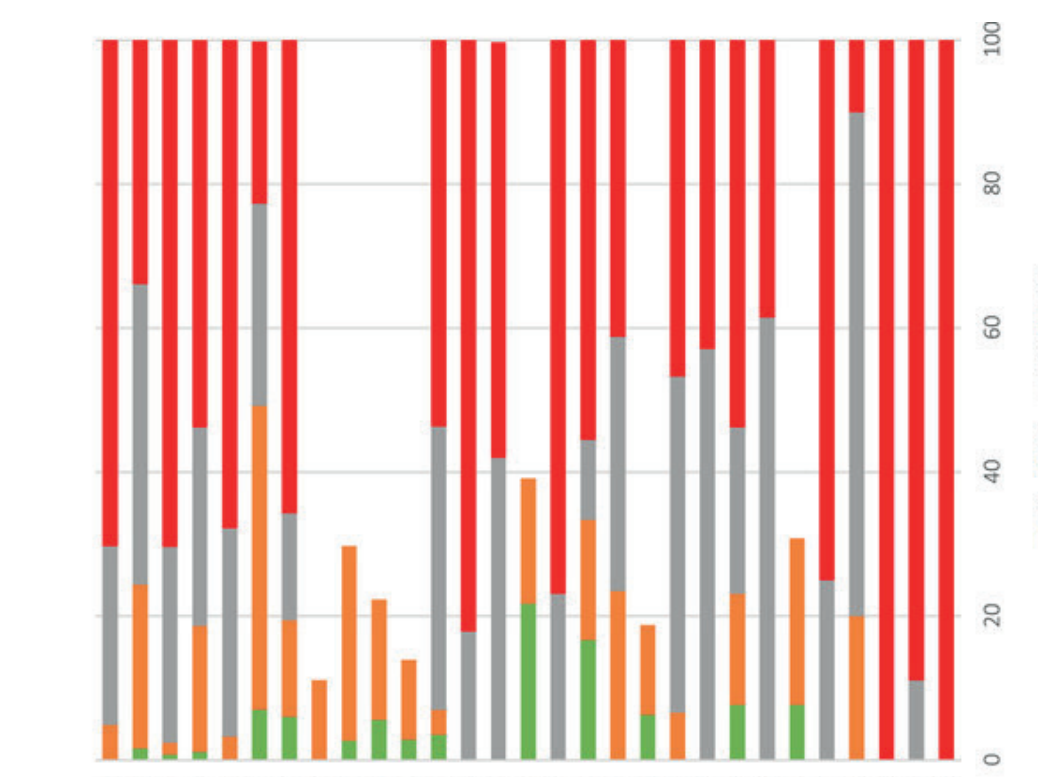

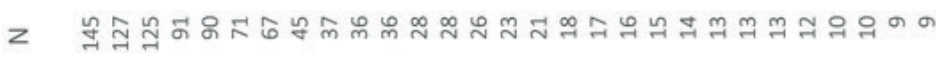
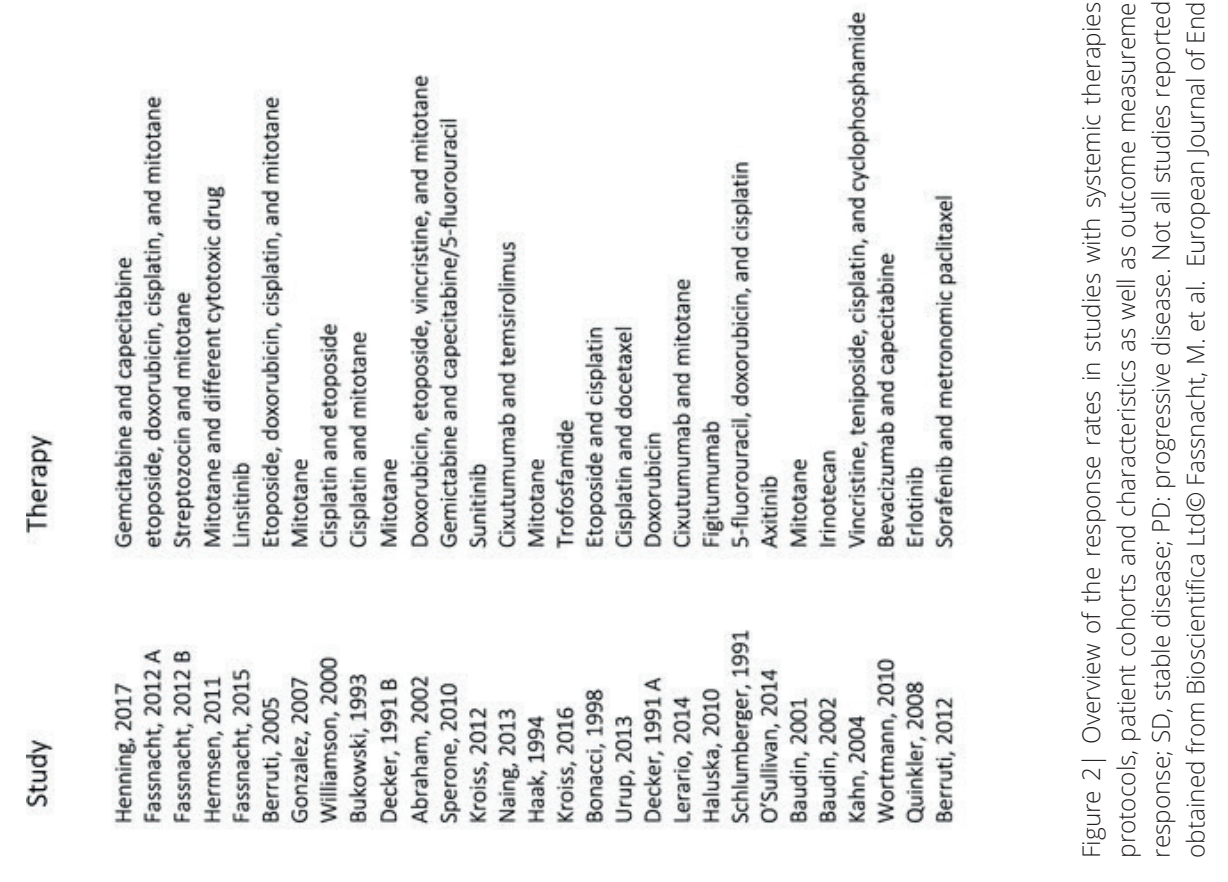


\section{Immunotherapy}

Immunotherapy is aimed at programmed cell death-1 (PD-1), an immune-checkpoint receptor expressed by $T$ cells, and programmed cell death ligand-1 and -2 (PD-L1 and PD-L2), expressed in the tumor microenvironment of various cancers. Recent research has shown that an estimated 11\% of ACCS express PD-L1 on tumor cell membranes, and $70 \%$ of tumor-infiltrating monocytes are PD-L1-positive (120). Immunotherapy has positively changed the prognosis significantly for patients with melanoma, renal cell carcinoma and lung cancer. Logically, multiple clinical trials are currently exploring the role of immunotherapy in ACC. So far results that have been published show mixed response, and there is a hypothesis that concomitant mitotane may be beneficial (121-124).

\section{Other}

Radiotherapy is used as a palliative therapy especially in symptomatic bone and brain metastases (125) and has been opted as adjuvant therapy of the tumor bed to reduce risk of local recurrence (126).

Data on data on loco-regional therapeutic options as hyperthermic intraperitoneal chemotherapy (HIPEC) (127) and transcatheter arterial chemoembolization (TACE) (128) is limited but show potential in selected patients with recurrent ACC confined to the peritoneal cavity or liver metastases respectively.

Radiofrequency ablation (RFA) was described in 8 ACC patients in which 15 lesions were ablated: 8 out of the 15 ablated tumors showed decreased tumor size (129) and is suggested suitable in cases with limited number of liver lesions (e. g. $\leq 3)$ with a diameter of less than $3 \mathrm{~cm}$.

Metformin, the anti-diabetic drug, has been associated with a decreased cancer prevalence and mortality in some solid tumors. It was shown to inhibit cell growth in an ACC experimental cell line (130). Peixoto et al described a case of female patient who achieved stable disease with mitotane, cisplatin, doxorubicin, and etoposide as first-line therapy, but then had an objective response to oral metformin that lasted 9 months(131) validating further research.

\section{Prognosis}

Median overall survival of ACC patients is about 3-4 years, the five-years overall survival being below $40 \%$. However, the prognosis is very heterogeneous and difficult to predict in clinical practice.

Great variability in clinical course is observed, ranging from tumors with an indolent 
behavior and patients with extreme long survival (132) to aggressive tumors with prompt fatal outcome. This heterogeneity in survival makes it complicated to tailor treatment strategies for an individual patient. Therefore, a lot of effort has been made to identify prognostic markers for survival

The extent of the disease at the time of diagnosis is considered a major determinant of survival. This can be assayed by the ENSAT staging score with a five-year stagedependent survival of 66-82\% for stage I, 58-64\% for stage II, 24-50\% for stage III, and $0-17 \%$ for stage IV(133). Approximately 30\% of patients are diagnosed with metastases at primary presentation (15). Within the group of patients with locally advanced disease survival mainly seem influenced by positive lymph nodes (N1) or vena cava invasion and could have a similar prognosis to those with stage IV disease $(61,134)$. The number of involved organs in patients with stage IV ACC has been reported to influence prognosis, a high number of involved organs means worse prognosis $(61,135)$.

Several studies showed that patients with cortisol-secreting ACC have a decreased overall survival. It has been hypothesized that excess cortisol poses multiple clinical challenges related to other comorbidities, such as hypertension, hyperglycemia, hypokalemia, bone loss, hypercoagulability and immune suppression (136-139).

Furthermore, several studies also claim that age, the Weiss score (a Weiss-score greater than six has been found to correspond with poor prognosis) index and the resection (R) status may impact prognosis of ACC patients $(61,140,141)$.

The Ki67 has consistently been proven to be associated with prognosis. A malignant tumor with a Ki67 index under 10\% is associated with a relatively good prognosis; an index over $20 \%$ is associated with a detrimental course of disease (142).

Molecular markers have been of increasing interest for prognostication of ACC. Pangenomic studies have been performed in ACC, showing distinct molecular subtypes with clinically relevant differences in outcome (143-145).

In particular two subgroups of ACCs, strongly correlated with survival, were identified by clustering of mRNA expression (145). The aggressive, high mutation rate, cluster C1A is associated with a poor outcome, which has been characterized by a specific transcriptomic signature, a methylome signature of hypermethylation on CpG islands called CIMP (146), a high number of chromosome alterations called noisy, and an accumulation of mutations among a limited number of recurrent driver genes $(143,147)$. The C1A ACCs can be classified into three subgroups according to methylation profile: CIMP-high, CIMP-low and non-CIMP $(143,146)$.

Conversely, another patient subgroup has been associated with a better outcome, the indolent C1B transcriptomic, generally non-CIMP, cluster $(143,144)$. 


\section{Aims and outline of this thesis}

To date, the European Society of Endocrinology Clinical Practice Guidelines Clinical recommends taking into account the factors tumor stage, resection status, Ki67 index, autonomous cortisol

secretion and the patient's general condition when assessing prognosis for patients with ACC (37). The acronym 'GRAS' has been put forward: Grading (G), the R status $(\mathrm{R})$, Age (A) and Symptoms (S), defined as tumor- or hormone-related symptoms at diagnosis to correspond to these parameters $(61,148)$. Still, using these parameters survival varies among patients complicating therapeutic decision-making. In order to gain insight into factors affecting prognosis, we analyzed the influence of different time patterns of metastases are associated with the outcome of patients with ACC in chapter 2. In addition, we aimed to study the effect of the number of metastases and affected organs on survival.

A prediction model combining the available prognostic parameters that have been individually associated with survival could be of significant additional value, and may be more useful in clinical practice. Therefore in chapter $\mathbf{3}$ a clinical prediction model for ACC-specific mortality is presented.

Mitotane is the only FDA approved drug treatment for ACC. At present, no available pharmacological options have been proven better than mitotane. Despite 60 years of use, many of its pharmacological properties, such as exact pharmacodynamics and activation in humans, remain not fully understood.

There is a large inter-patient variability in reaching and maintaining therapeutic concentrations, without a clear relationship between the mitotane dose and the serum concentration. The variability in mitotane requirement that cannot be explained by the clinical factors, in other words the residual variability, may very well be explained by pharmacogenetic differences between patients. Identifying these differences and designing a pharmacokinetic model in which such pharmacogenetic differences are taken into account could allow a more individualized elegant treatment of ACC with mitotane.

Recent study has shown considerably weak correlations between weight (both total weight and Lean Body Mass) and the volume of distribution in central compartment (V1) of mitotane. Other covariates (age, gender, height, BodySurfaceArea) displayed correlations in the same order of magnitude (84). These findings have led to the hypothesis that differences in genetic constitution, are of significant importance. Indeed, recent research states that patients with a certain genotype of the CYP2B6 gene show higher mitotane plasma concentrations at three months of treatment, which supports this hypothesis (149). Mitotane concentrations were not influenced by the polymorphisms of the ABCB1 gene. Other genetic polymorphisms need further exploration. In chapter $\mathbf{4}$ pharmacogenetic factors or single nucleotide 
polymorphisms are investigated using the Drug Metabolizing Enzymes and Transporters (DMET) Platform. Furthermore a population pharmacokinetic (PopPK) model is described, allowing optimal treatment schedules for individual patients.

ACC management remains challenging because of the heterogeneous and often unpredictable nature of this disease. Advances in molecular analysis have enhanced the molecular understanding of ACC. Unfortunately, this has not yielded a major therapeutic breakthrough and EDP-M remains the main systemic therapy option for many patients. However, emerging genomic techniques may be able to identify cancer dependencies and select priority drug candidates in the near future. In chapter 5 a view is presented on the future of ACC treatment that is expected to be brighter than it has been so far. Furthermore, ACC diagnostics and prognostics is discussed since significant changes in these fields are expected contributing to new treatment strategies.

Epigenetic regulatory mechanisms have emerged as clinically relevant diagnostic and prognostic factors in ACC. DNA methylation levels and CIMP-phenotypes have shown to be able to stratify ACC prognostic subgroups. The therapeutic potential of epigenetic alterations in ACC is unclear and should be further investigated. In chapter 6 an overview of new insights in the evolving field of epigenetic studies on adrenal tumors is provided. 


\section{References}

1. Singh V. General Anatomy-E-book. : Elsevier Health Sciences; 2015.

2. Melmed S, Polonsky KS, Larsen PR, Kronenberg HM. Williams textbook of endocrinology. : Elsevier Health Sciences; 2015.

3. Young WF,Jr. Clinical practice. The incidentally discovered adrenal mass. N Engl J Med 2007 02/08;356(1533-4406; 6):601-610

4. Barzon L, Sonino N, Fallo F, Palu G, Boscaro M. Prevalence and natural history of adrenal incidentalomas. Eur J Endocrinol 2003 Oct;149(4):273-285.

5. Terzolo M, Stigliano A, Chiodini I, Loli P, Furlani L, Arnaldi G, et al. AME position statement on adrenal incidentaloma. Eur J Endocrinol 2011 Jun;164(6):851-870.

6. Fassnacht M, Allolio B. Clinical management of adrenocortical carcinoma. Best Pract Res Clin Endocrinol Metab 2009 04;23(1532-1908; 2):273-289.

7. Kebebew E, Reiff E, Duh QY, Clark OH, McMillan A. Extent of disease at presentation and outcome for adrenocortical carcinoma: have we made progress? World J Surg 2006 May;30(5):872-878.

8. Third national cancer survey: incidence data. Natl Cancer Inst Monogr 1975 Mar;(41)(41):i-X, $1-454$.

9. Kerkhofs TM, Verhoeven RH, Van der Zwan JM, Dieleman J, Kerstens MN, Links TP, et al. Adrenocortical carcinoma: a population-based study on incidence and survival in the Netherlands since 1993. Eur J Cancer 2013 Jul;49(11):2579-2586.

10. Wajchenberg BL, Albergaria Pereira MA, Medonca BB, Latronico AC, Campos CP, Alves VA, et al. Adrenocortical carcinoma: clinical and laboratory observations. Cancer 2000 02/15;88(0008-543; 4):711-736.

11. Wooten MD, King DK. Adrenal cortical carcinoma. Epidemiology and treatment with mitotane and a review of the literature. Cancer 1993 12/01;72(0008-543; 11):3145-3155.

12. Else T, Kim AC, Sabolch A, Raymond VM, Kandathil A, Caoili EM, et al. Adrenocortical carcinoma. Endocr Rev 2013;35(2):282-326.

13. Sandrini R, Ribeiro RC, DeLacerda L. Childhood adrenocortical tumors. J Clin Endocrinol Metab 1997 Jul;82(7):2027-2031.

14. Ribeiro RC, Sandrini F, Figueiredo B, Zambetti GP, Michalkiewicz E, Lafferty AR, et al. An inherited p53 mutation that contributes in a tissue-specific manner to pediatric adrenal cortical carcinoma. Proc Natl Acad Sci U S A 2001 Jul 31;98(16):9330-9335.

15. Koschker AC, Fassnacht M, Hahner S, Weismann D, Allolio B. Adrenocortical carcinoma -- improving patient care by establishing new structures. Exp Clin Endocrinol Diabetes 2006 02;114(09477349; 0947-7349; 2):45-51.

16. Gatta-Cherifi B, Chabre O, Murat A, Niccoli P, Cardot-Bauters C, Rohmer V, et al. Adrenal involvement in MEN1. Analysis of 715 cases from the Groupe d'etude des Tumeurs Endocrines database. European journal of endocrinology 2012;166(2):269-279.

17. Li FP, Fraumeni JF, Mulvihill J], Blattner WA, Dreyfus MG, Tucker MA, et al. A cancer family syndrome in twenty-four kindreds. Cancer Res 1988;48(18):5358-5362.

18. Karamurzin Y, Zeng Z, Stadler ZK, Zhang L, Ouansafi I, Al-Ahmadie HA, et al. Unusual DNA mismatch repair-deficient tumors in Lynch syndrome: a report of new cases and review of the literature. Hum Pathol 2012;43(10):1677-1687.

19. Beckwith-wiedemann syndrome. American Journal of Medical Genetics Part C: Seminars in Medical Genetics: Wiley Online Library; 2005. 
20. Wiedemann $\mathrm{H}$. Tumours and hemihypertrophy associated with Wiedemann-Beckwith syndrome. Eur J Pediatr 1983;141(2):129-129.

21. Gaujoux S, Pinson S, Gimenez-Roqueplo AP, Amar L, Ragazzon B, Launay P, et al. Inactivation of the APC gene is constant in adrenocortical tumors from patients with familial adenomatous polyposis but not frequent in sporadic adrenocortical cancers. Clin Cancer Res 2010 Nov 1;16(21):5133-5141.

22. Else T. Association of adrenocortical carcinoma with familial cancer susceptibility syndromes. Mol Cell Endocrinol 2012;351(1):66-70.

23. Takazawa R, Ajima J, Yamauchi A, Goto M. Unusual double primary neoplasia: adrenocortical and ureteral carcinomas in werner syndrome. Urol Int 2004;72(2):168-170.

24. Lerario AM, Moraitis A, Hammer GD. Genetics and epigenetics of adrenocortical tumors. Mol Cell Endocrinol 2014 Apr 5;386(1-2):67-84.

25. Weksberg R, Shuman C, Beckwith JB. Beckwith-Wiedemann syndrome. European journal of human genetics 2010;18(1):8.

26. Fassnacht M, Libe R, Kroiss M, Allolio B. Adrenocortical carcinoma: a clinician's update. Nat Rev Endocrinol 2011 Jun; 7(6):323-335.

27. Bonnet-Serrano F, Bertherat J. Genetics of tumors of the adrenal cortex. Endocr Relat Cancer 2018;25(3):R131-R152.

28. Varghese J, Habra MA. Update on adrenocortical carcinoma management and future directions. Curr Opin Endocrinol Diabetes Obes 2017 Jun;24(3):208-214.

29. Giordano TJ, Thomas DG, Kuick R, Lizyness M, Misek DE, Smith AL, et al. Distinct transcriptional profiles of adrenocortical tumors uncovered by DNA microarray analysis. Am J Pathol 2003 02;162(0002-9440; 2):521-531.

30. Gicquel C, Bertagna X, Gaston V, Coste J, Louvel A, Baudin E, et al. Molecular markers and longterm recurrences in a large cohort of patients with sporadic adrenocortical tumors. Cancer Res 2001 09/15;61(0008-5472; 18):6762-6767.

31. Gaujoux S, Grabar S, Fassnacht M, Ragazzon B, Launay P, Libe R, et al. Beta-Catenin Activation is Associated with Specific Clinical and Pathologic Characteristics and a Poor Outcome in Adrenocortical Carcinoma. Clin Cancer Res 2011 Jan 15;17(2):328-336.

32. Raymond VM, Else T, Everett JN, Long JM, Gruber SB, Hammer GD. Prevalence of germline TP53 mutations in a prospective series of unselected patients with adrenocortical carcinoma. The Journal of Clinical Endocrinology \& Metabolism 2013;98(1):E119-E125.

33. Wasserman JD, Zambetti GP, Malkin D. Towards an understanding of the role of p53 in adrenocortical carcinogenesis. Mol Cell Endocrinol 2012 Mar 31;351(1):101-110.

34. Chompret A, Abel A, Stoppa-Lyonnet D, Brugieres L, Pages S, Feunteun J, et al. Sensitivity and predictive value of criteria for p53 germline mutation screening. J Med Genet 2001 Jan;38(1):43-47.

35. Hsing AW, Nam J, Co Chien HT, McLaughlin JK, Fraumeni Jr JF. Risk factors for adrenal cancer: an exploratory study. International journal of cancer 1996;65(4):432-436.

36. Allolio B, Fassnacht M. Clinical review: Adrenocortical carcinoma: clinical update. J Clin Endocrinol Metab 2006 06;91(0021-972; 6):2027-2037.

37. Fassnacht M, Dekkers O, Else T, Baudin E, Berruti A, De Krijger RR, et al. European Society of Endocrinology Clinical Practice Guidelines on the management of adrenocortical carcinoma in adults, in collaboration with the European Network for the Study of Adrenal Tumors. European journal of endocrinology 2018;1(aop).

38. Dinnes J, Bancos I, Di Ruffano LF, Chortis V, Davenport C, Bayliss S, et al. Management of endocrine disease: imaging for the diagnosis of malignancy in incidentally discovered adrenal masses: a 
systematic review and meta-analysis. European journal of endocrinology 2016;175(2):R51-R64.

39. Caoili EM, Korobkin M, Francis IR, Cohan RH, Platt JF, Dunnick NR, et al. Adrenal masses: characterization with combined unenhanced and delayed enhanced CT. Radiology 2002 Mar;222(3):629-633.

40. Pena CS, Boland GW, Hahn PF, Lee MJ, Mueller PR. Characterization of indeterminate (lipidpoor) adrenal masses: use of washout characteristics at contrast-enhanced CT. Radiology 2000 Dec;217(3):798-802.

41. Szolar DH, Korobkin M, Reittner P, Berghold A, Bauernhofer T, Trummer H, et al. Adrenocortical carcinomas and adrenal pheochromocytomas: mass and enhancement loss evaluation at delayed contrast-enhanced CT. Radiology 2005 Feb;234(2):479-485.

42. Caoili EM, Korobkin M, Brown RK, Mackie G, Shulkin BL. Differentiating adrenal adenomas from nonadenomas using 18F-FDG PET/CT: quantitative and qualitative evaluation. Acad Radio 2007;14(4):468-475.

43. Zini L, Porpiglia F, Fassnacht M. Contemporary management of adrenocortical carcinoma. Eur Urol 2011;60(5):1055-1065.

44. Kerkhofs TM, Kerstens MN, Kema IP, Willems TP, Haak HR. Diagnostic Value of Urinary Steroid Profiling in the Evaluation of Adrenal Tumors. Horm Cancer 2015 Aug;6(4):168-175.

45. Arlt W, Biehl M, Taylor AE, Hahner S, Libe R, Hughes BA, et al. Urine steroid metabolomics as a biomarker tool for detecting malignancy in adrenal tumors. J Clin Endocrinol Metab 2011 Dec;96(12):3775-3784.

46. Minowada S, Kinoshita K, Hara M, Isurugi K, Uchikawa T, Niijima T. Measurement of urinary steroid profile in patients with adrenal tumor as a screening method for carcinoma. Endocrinol Jpn 1985 Feb;32(1):29-37.

47. Gröndal S, Eriksson B, Hagenas L, Werner S, Curstedt T. Steroid profile in urine: a useful tool in the diagnosis and follow up of adrenocortical carcinoma. Acta Endocrinol (Copenh) 1990 May; 122(5):656-663.

48. Kikuchi E, Yanaihara H, Nakashima J, Homma K, Ohigashi T, Asakura H, et al. Urinary steroid profile in adrenocortical tumors. Biomed Pharmacother 2000 Jun;54 Suppl 1:194s-197s.

49. van Slooten H, Schaberg A, Smeenk D, Moolenaar AJ. Morphologic characteristics of benign and malignant adrenocortical tumors. Cancer 1985 Feb 15;55(4):766-773.

50. Weiss LM. Comparative histologic study of 43 metastasizing and nonmetastasizing adrenocortical tumors. Am J Surg Pathol 1984 03;8(0147-5185; 3):163-169.

51. Fuhrman SA, Lasky LC, Limas C. Prognostic significance of morphologic parameters in renal cell carcinoma. Am J Surg Pathol 1982;6(7):655-663.

52. Weiss LM, Medeiros LJ, Vickery AL,Jr. Pathologic features of prognostic significance in adrenocortical carcinoma. Am J Surg Pathol 1989 03;13(0147-5185; 3):202-206.

53. Wang C, Sun Y, Wu H, Zhao D, Chen J. Distinguishing adrenal cortical carcinomas and adenomas: a study of clinicopathological features and biomarkers. Histopathology 2014;64(4):567-576.

54. Sbiera S, Schmull S, Assie G, Voelker H, Kraus L, Beyer M, et al. High diagnostic and prognostic value of steroidogenic factor-1 expression in adrenal tumors. The Journal of Clinical Endocrinology \& Metabolism 2010;95(10):E161-E171.

55. Nakazumi H, Sasano H, lino K, Ohashi Y, Orikasa S. Expression of cell cycle inhibitor p27 and Ki-67 in human adrenocortical neoplasms. Modern pathology: an official journal of the United States and Canadian Academy of Pathology, Inc 1998;11(12):1165-1170.

56. Terzolo M, Boccuzzi A, Bovio S, Cappia S, De Giuli P, Ali A, et al. Immunohistochemical assessment 
of Ki-67 in the differential diagnosis of adrenocortical tumors. Urology 2001 Jan;57(1):176-182.

57. Pinzani P, Scatena C, Salvianti F, Corsini E, Canu L, Poli G, et al. Detection of circulating tumor cells in patients with adrenocortical carcinoma: a monocentric preliminary study. J Clin Endocrinol Metab 2013 Sep;98(9):3731-3738.

58. Creemers SG, Korpershoek E, Atmodimedjo PN, Dinjens WNM, van Koetsveld PM, Feelders RA, et al. Identification of Mutations in Cell-Free Circulating Tumor DNA in Adrenocortical Carcinoma: A Case Series. J Clin Endocrinol Metab 2017 Oct 1;102(10):3611-3615.

59. Garinet $S$, Nectoux J, Neou M, Pasmant E, Jouinot A, Sibony M, et al. Detection and monitoring of circulating tumor DNA in adrenocortical carcinoma. Endocr Relat Cancer 2018;25(3):L13-L17.

60. Fassnacht M, Johanssen S, Quinkler M, Bucsky P, Willenberg HS, Beuschlein F, et al. Limited prognostic value of the 2004 International Union Against Cancer staging classification for adrenocortical carcinoma: proposal for a Revised TNM Classification. Cancer 2009 01/15;115(0008-543; 0008-543; 2):243-250.

61. Libe R, Borget I, Ronchi CL, Zaggia B, Kroiss M, Kerkhofs T, et al. Prognostic factors in stage III-IV adrenocortical carcinomas (ACC): an European Network for the Study of Adrenal Tumor (ENSAT) study. Ann Oncol 2015 Oct;26(10):2119-2125.

62. Donatini G, Caiazzo R, Do Cao C, Aubert S, Zerrweck C, El-Kathib Z, et al. Long-term survival after adrenalectomy for stage I/II adrenocortical carcinoma (ACC): a retrospective comparative cohort study of laparoscopic versus open approach. Ann Surg Oncol 2014 Jan;21(1):284-291.

63. Mir MC, Klink JC, Guillotreau J, Long JA, Miocinovic R, Kaouk JH, et al. Comparative outcomes of laparoscopic and open adrenalectomy for adrenocortical carcinoma: single, high-volume center experience. Ann Surg Oncol 2013 May;20(5):1456-1461.

64. Grubbs EG, Callender GG, Xing Y, Perrier ND, Evans DB, Phan AT, et al. Recurrence of adrenal cortical carcinoma following resection: surgery alone can achieve results equal to surgery plus mitotane. Ann Surg Oncol 2010 01;17(1534-4681; 1068-9265; 1):263-270.

65. Terzolo M, Angeli A, Fassnacht M, Daffara F, Tauchmanova L, Conton PA, et al. Adjuvant mitotane treatment for adrenocortical carcinoma. N EngI J Med 2007 06/07;356(1533-4406; 23):2372-2380.

66. Margonis GA, Kim Y, Prescott JD, Tran TB, Postlewait LM, Maithel SK, et al. Adrenocortical carcinoma: impact of surgical margin status on long-term outcomes. Annals of surgical oncology 2016;23(1):134-141.

67. Dy BM, Wise KB, Richards ML, Young WF,Jr, Grant CS, Bible KC, et al. Operative intervention for recurrent adrenocortical cancer. Surgery 2013 Dec;154(6):1292-9; discussion 1299.

68. Erdogan I, Deutschbein T, Jurowich C, Kroiss M, Ronchi C, Quinkler M, et al. The role of surgery in the management of recurrent adrenocortical carcinoma. J Clin Endocrinol Metab 2013 Jan;98(1):181-191.

69. Livhits M, Li N, Yeh MW, Harari A. Surgery is associated with improved survival for adrenocortical cancer, even in metastatic disease. Surgery 2014 Dec;156(6):1531-40; discussion 1540-1.

70. Prendergast KM, Smith PM, Tran TB, Postlewait LM, Maithel SK, Prescott JD, et al. Features of synchronous versus metachronous metastasectomy in adrenal cortical carcinoma: Analysis from the US adrenocortical carcinoma database. Surgery 2019.

71. Kemp CD, Ripley RT, Mathur A, Steinberg SM, Nguyen DM, Fojo T, et al. Pulmonary resection for metastatic adrenocortical carcinoma: the National Cancer Institute experience. Ann Thorac Surg 2011 Oct;92(4):1195-1200.

72. NELSON AA, WOODARD G. Adrenal cortical atrophy and liver damage produced in dogs by feeding 2,2-bis-(parachloro-phenyl)-1,1-dichloroethane. Fed Proc 1948 03;7(0014-9446; 1):277. 
73. Touitou Y, Bogdan A, Luton JP. Changes in corticosteroid synthesis of the human adrenal cortex in vitro, induced by treatment with o, $p^{\prime}-\mathrm{DDD}$ for Cushing's syndrome: evidence for the sites of action of the drug. J Steroid Biochem 1978 Dec;9(12):1217-1224.

74. Bergenstal D, Hertz R, Lipsett M, Moy R. Chemotherapy of adrenocortical cancer with o,p'-DDD. 1960(53):672.

75. Leboulleux S, Chadarevian R, Trabado S, Amazit L. Mitotane alters mitochondrial respiratory chain activity by inducing cytochrome c oxidase defect in human adrenocortical cells. Endocr Relat Cancer 2013;20:371-381.

76. Poli G, Guasti D, Rapizzi E, Fucci R, Canu L, Bandinelli A, et al. Morphofunctional effects of mitotane on mitochondria in human adrenocortical cancer cells. Endocr Relat Cancer 2013;20(4):537-550.

77. Sbiera S, Leich E, Liebisch G, Sbiera I, Schirbel A, Wiemer L, et al. Mitotane inhibits sterol-Oacyl transferase 1 triggering lipid-mediated endoplasmic reticulum stress and apoptosis in adrenocortical carcinoma cells. Endocrinology 2015;156(11):3895-3908.

78. Moolenaar AJ, van SH, van Seters AP, Smeenk D. Blood levels of o,p'-DDD following administration in various vehicles after a single dose and during long-term treatment. Cancer Chemother Pharmacol 1981;7(0344-5704; 1):51-54.

79. von Slooten H, van Seters AP, Smeenk D, Moolenaar AJ. O,p'-DDD (mitotane) levels in plasma and tissues during chemotherapy and at autopsy. Cancer Chemother Pharmacol 1982;9(2):85-88.

80. Hermsen IG, Fassnacht M, Terz. Plasma concentrations of o,p'DDD, o,p'DDA, and o,p'DDE as predictors of tumor response to mitotane in adrenocortical carcinoma: results of a retrospective ENS@T multicenter study. 2011 Jun;96(6):1844-1851.

81. Haak HR, Hermans J, van de Velde CJ, Lentjes EG, Goslings BM, Fleuren GJ, et al. Optimal treatment of adrenocortical carcinoma with mitotane: results in a consecutive series of 96 patients. $\mathrm{Br}\rfloor$ Cancer 1994 05;69(0007-0920; 5):947-951.

82. Moy RH. Studies of the pharmacology of o,p'DDD in man. J Lab Clin Med 1961 08;58(0022-2143; 0022-2143):296-304.

83. Arshad U, Taubert M, Kurlbaum M, Frechen S, Herterich S, Megerle F, et al. Enzyme autoinduction by mitotane supported by population pharmacokinetic modeling in a large cohort of adrenocortical carcinoma patients. European journal of endocrinology 2018;179(5):287-297.

84. Kerkhofs TM, Derijks LJ, Ettaieb H, den Hartigh J, Neef K, Gelderblom H, et al. Development of a pharmacokinetic model of mitotane: toward personalized dosing in adrenocortical carcinoma. Ther Drug Monit 2015 Feb;37(1):58-65.

85. Kerkhofs TM, Baudin E, Terzolo M, Allolio B, Chadarevian R, Mueller HH, et al. Comparison of two mitotane starting dose regimens in patients with advanced adrenocortical carcinoma. J Clin Endocrinol Metab 2013 Dec;98(12):4759-4767.

86. Kerkhofs TM, Derijks LJ, Ettaieb MH, Eekhoff EM, Neef C, Gelderblom H, et al. Short-term variation in plasma mitotane levels confirms the importance of trough level monitoring. Eur J Endocrinol 2014 Dec;171(6):677-683.

87. Daffara F, De Francia S, Reimondo G, Zaggia B, Aroasio E, Porpiglia F, et al. Prospective evaluation of mitotane toxicity in adrenocortical cancer patients treated adjuvantly. Endocr Relat Cancer 2008 Dec;15(4):1043-1053.

88. European Medicines Agency. Lysodren: EPAR - scientific discussion. 2006; Available at: https:// www.ema.europa.eu. Accessed 01/14, 2020.

89. (89) Salenave S, Bernard V, Do Cao C, Guignat L, Bachelot A, Leboulleux S, et al. Ovarian macrocysts and gonadotrope-ovarian axis disruption in premenopausal women receiving mitotane for adrenocortical carcinoma or Cushing's disease. Eur J Endocrinol 2015 Feb;172(2):141-149. 
90. Calabrese A, Basile V, Puglisi S, Perotti P, Pia A, Saba L, et al. Adjuvant mitotane therapy is beneficial in non-metastatic adrenocortical carcinoma at high risk of recurrence. European journal of endocrinology 2019;180(6):387-396.

91. Hahner S, Fassnacht M. Mitotane for adrenocortical carcinoma treatment. Curr Opin Investig Drugs 2005 04;6(1472-4472; 4):386-394.

92. NICHOLS J, HENNIGAR G. Studies on DDD, 2,2-bis (parachlorophenyl)-1,1-dichloroethane. Exp Med Surg 1957;15(4):310-316.

93. CUETO C, BROWN JH, RICHARDSON AP,Jr. Biological studies on an adrenocorticolytic agent and the isolation of the active components. Endocrinology 1958 Mar;62(3):334-339.

94. Fassnacht M, Terzolo M, Allolio B, Baudin E, Haak H, Berruti A, et al. Combination Chemotherapy in Advanced Adrenocortical Carcinoma. N Engl J Med 2012 05/02; 2012/05.

95. Jasim S, Habra MA. Management of adrenocortical carcinoma. Curr Oncol Rep 2019;21(3):20.

96. Henning JE, Deutschbein T, Altieri B, Steinhauer S, Kircher S, Sbiera S, et al. Gemcitabine-based chemotherapy in adrenocortical carcinoma: a multicenter study of efficacy and predictive factors. The Journal of Clinical Endocrinology \& Metabolism 2017;102(11):4323-4332.

97. Fassnacht M, Berruti A, Baudin E, Demeure MJ, Gilbert J, Haak H, et al. Linsitinib (OSI-906) versus placebo for patients with locally advanced or metastatic adrenocortical carcinoma: a doubleblind, randomised, phase 3 study. Lancet Oncol 2015 Apr;16(4):426-435.

98. Berruti A, Terzolo M, Sperone P, Pia A, Casa SD, Gross DJ, et al. Etoposide, doxorubicin and cisplatin plus mitotane in the treatment of advanced adrenocortical carcinoma: a large prospective phase II trial. Endocr Relat Cancer 2005 09;12(1351-0088; 3):657-666.

99. Gonzalez RJ, Tamm EP, Ng C, Phan AT, Vassilopoulou-Sellin R, Perrier ND, et al. Response to mitotane predicts outcome in patients with recurrent adrenal cortical carcinoma. Surgery 2007 $12 ; 142(1532-7361 ; 6): 867-875$

100. Williamson SK, Lew D, Miller GJ, Balcerzak SP, Baker LH, Crawford ED. Phase II evaluation of cisplatin and etoposide followed by mitotane at disease progression in patients with locally advanced or metastatic adrenocortical carcinoma: a Southwest Oncology Group Study. Cancer 2000 03/01;88(0008-543; 5):1159-1165.

101. Bukowski RM, Wolfe M, Levine HS, Crawford DE, Stephens RL, Gaynor E, et al. Phase II trial of mitotane and cisplatin in patients with adrenal carcinoma: a Southwest Oncology Group study. J Clin Oncol 1993 01;11(0732-183; 1):161-165.

102. Decker RA, Elson P, Hogan TF, Citrin DL, Westring DW, Banerjee TK, et al. Eastern Cooperative Oncology Group study 1879: mitotane and adriamycin in patients with advanced adrenocortical carcinoma. Surgery 1991 12;110(0039-6060; 6):1006-1013.

103. Abraham J, Bakke S, Rutt A, Meadows B, Merino M, Alexander R, et al. A phase II trial of combination chemotherapy and surgical resection for the treatment of metastatic adrenocortical carcinoma: continuous infusion doxorubicin, vincristine, and etoposide with daily mitotane as a P-glycoprotein antagonist. Cancer 2002 05/01;94(0008-543; 9):2333-2343.

104. Sperone P, Ferrero A, Daffara F, Priola A, Zaggia B, Volante M, et al. Gemcitabine plus metronomic 5-fluorouracil or capecitabine as a second-/third-line chemotherapy in advanced adrenocortical carcinoma: a multicenter phase II study. Endocr Relat Cancer 2010 Apr 21;17(2):445-453.

105. Kroiss M, Quinkler M, Johanssen S, van Erp NP, Lankheet N, Pollinger A, et al. Sunitinib in refractory adrenocortical carcinoma: a phase II, single-arm, open-label trial. J Clin Endocrinol Metab 2012 Oct;97(10):3495-3503.

106. Naing A, Lorusso P, Fu S, Hong D, Chen HX, Doyle LA, et al. Insulin growth factor receptor (IGF$1 \mathrm{R})$ antibody cixutumumab combined with the mTOR inhibitor temsirolimus in patients with 
metastatic adrenocortical carcinoma. Br J Cancer 2013 Mar 5;108(4):826-830.

107. Kroiss M, Deutschbein T, Schlötelburg W, Ronchi CL, Neu B, Müller $H$, et al. Salvage treatment of adrenocortical carcinoma with trofosfamide. Hormones and Cancer 2016;7(3):211-218.

108. Bonacci R, Gigliotti A, Baudin E, Wion-Barbot N, Emy P, Bonnay M, et al. Cytotoxic therapy with etoposide and cisplatin in advanced adrenocortical carcinoma. Reseau Comete INSERM. Br J Cancer 1998 08;78(0007-0920; 4):546-549.

109. Urup T, Pawlak WZ, Petersen PM, Pappot H, Rorth M, Daugaard G. Treatment with docetaxel and cisplatin in advanced adrenocortical carcinoma, a phase II study. Br J Cancer 2013 May 28;108(10):1994-1997.

110. Lerario AM, Worden FP, Ramm CA, Hasseltine EA, Stadler WM, Else T, et al. The combination of insulin-like growth factor receptor 1 (IGF1R) antibody cixutumumab and mitotane as a firstline therapy for patients with recurrent/metastatic adrenocortical carcinoma: a multi-institutional NCl-sponsored trial. Hormones and Cancer 2014;5(4):232-239.

111. Haluska P, Worden F, Olmos D, Yin D, Schteingart D, Batzel GN, et al. Safety, tolerability, and pharmacokinetics of the anti-IGF-1R monoclonal antibody figitumumab in patients with refractory adrenocortical carcinoma. Cancer Chemother Pharmacol 2010;65(4):765-773.

112. Schlumberger M, Brugieres L, Gicquel C, Travagli JP, Droz JP, Parmentier C. 5-Fluorouracil, doxorubicin, and cisplatin as treatment for adrenal cortical carcinoma. Cancer 1991 06/15;67(0008-543; 12):2997-3000.

113. O'Sullivan C, Edgerly M, Velarde M, Wilkerson J, Venkatesan AM, Pittaluga S, et al. The VEGF inhibitor axitinib has limited effectiveness as a therapy for adrenocortical cancer. The Journal of Clinical Endocrinology \& Metabolism 2014;99(4):1291-1297.

114. Baudin E, Pellegriti G, Bonnay M, Penfornis A, Laplanche A, Vassal G, et al. Impact of monitoring plasma 1,1-dichlorodiphenildichloroethane (o,p'DDD) levels on the treatment of patients with adrenocortical carcinoma. Cancer 2001 09/15;92(0008-543; 6):1385-1392.

115. Baudin E, Docao C, Gicquel C, Vassal G, Bachelot A, Penfornis A, et al. Use of a topoisomerase I inhibitor (irinotecan, CPT-11) in metastatic adrenocortical carcinoma. Ann Oncol 2002 11;13(09237534; 11):1806-1809.

116. Khan TS, Sundin A, Juhlin C, Wilander E, Oberg K, Eriksson B. Vincristine, cisplatin, teniposide, and cyclophosphamide combination in the treatment of recurrent or metastatic adrenocortical cancer. Med Oncol 2004;21(1357-0560; 2):167-177.

117. Wortmann S, Quinkler M, Ritter C, Kroiss M, Johanssen S, Hahner S, et al. Bevacizumab plus capecitabine as a salvage therapy in advanced adrenocortical carcinoma. Eur J Endocrinol 2010 Feb;162(2):349-356.

118. Quinkler M, Hahner S, Wortmann S, Johanssen S, Adam P, Ritter C, et al. Treatment of advanced adrenocortical carcinoma with erlotinib plus gemcitabine. J Clin Endocrinol Metab 2008 Jun;93(6):2057-2062.

119. Berruti A, Sperone P, Ferrero A, Germano A, Ardito A, Priola AM, et al. Phase II study of weekly paclitaxel and sorafenib as second/third line therapy in patients with adrenocortical carcinoma. Eur J Endocrinol 2011 Dec 21.

120. Fay AP, Signoretti S, Callea M, Telomicron GH, McKay RR, Song J, et al. Programmed death ligand-1 expression in adrenocortical carcinoma: an exploratory biomarker study. J Immunother Cancer 2015 Feb 17;3:3-015-0047-3. eCollection 2015.

121. Habra MA, Stephen B, Campbell M, Hess K, Tapia C, Xu M, et al. Phase ll clinical trial of pembrolizumab efficacy and safety in advanced adrenocortical carcinoma. Journal for immunotherapy of cancer 2019;7(1):253. 
122. Le Tourneau C, Hoimes C, Zarwan C, Wong DJ, Bauer S, Claus R, et al. Avelumab in patients with previously treated metastatic adrenocortical carcinoma: phase $1 \mathrm{~b}$ results from the JAVELIN solid tumor trial. Journal for immunotherapy of cancer 2018;6(1):111.

123. Head L, Kiseljak-Vassiliades K, Clark TJ, Somerset H, King J, Raeburn C, et al. Response to Immunotherapy in Combination With Mitotane in Patients With Metastatic Adrenocortical Cancer. Journal of the Endocrine Society 2019;3(12):2295-2304.

124. Cavalcante L, Carneiro BA, Costa RLB, Chae YK, Rademaker A, Giles FJ. Preliminary results from a phase II study of nivolumab for patients with metastatic adrenocortical carcinoma (ACC). 2017.

125. Milgrom SA, Goodman KA. The role of radiation therapy in the management of adrenal carcinoma and adrenal metastases. J Surg Oncol 2012 Apr 4.

126. Fassnacht M, Hahner S, Polat B, Koschker AC, Kenn W, Flentje M, et al. Efficacy of adjuvant radiotherapy of the tumor bed on local recurrence of adrenocortical carcinoma. J Clin Endocrinol Metab 2006 11;91(0021-972; 11):4501-4504.

127. Hughes MS, Lo WM, Beresnev T, Merino M, Shutack Y, Ripley RT, et al. A phase II trial of cytoreduction and hyperthermic intraperitoneal chemotherapy for recurrent adrenocortical carcinoma. J Surg Res 2018;232:383-388.

128. Cazejust J, De Baere T, Auperin A, Deschamps F, Hechelhammer L, Abdel-Rehim M, et al. Transcatheter arterial chemoembolization for liver metastases in patients with adrenocortical carcinoma. J Vasc Interv Radiol 2010 Oct;21(10):1527-1532.

129. (129) Wood BJ, Abraham J, Hvizda JL, Alexander HR, Fojo T. Radiofrequency ablation of adrenal tumors and adrenocortical carcinoma metastases. Cancer 2003 02/01;97(0008-543; 3):554-560.

130. Poli G, Cantini G, Armignacco R, Fucci R, Santi R, Canu L, et al. Metformin as a new anti-cancer drug in adrenocortical carcinoma. Oncotarget 2016 Aug 2;7(31):49636-49648.

131. Peixoto R, Gomes L, Sousa T, Racy D, Shigenaga M, Nagourney R. Efficacy of oral metformin in a patient with metastatic adrenocortical carcinoma: Examination of mechanisms and therapeutic implications. Rare tumors 2018;10:2036361317749645.

132. Hermsen IG, Haak HR, de Krijger RR, Kerkhofs TM, Feelders RA, de Herder WW, et al. Mutationa analyses of epidermal growth factor receptor and downstream pathways in adrenocortical carcinoma. Eur J Endocrinol 2013 Jun 1;169(1):51-58.

133. Libé R. Adrenocortical carcinoma (ACC): diagnosis, prognosis, and treatment. Frontiers in cell and developmental biology 2015;3:45.

134. Do CC, Leboulleux S, Borget I, Bertherat J, De la Fouchardiere C, Hecart A, et al. First prognostic analysis of stage III adrenocartical carcinoma patients after complete resection: a retrospective French Multicentric Study from the INCA-COMETE network. 2011.

135. Assie G, Antoni G, Tissier F, Caillou B, Abiven G, Gicquel C, et al. Prognostic parameters of metastatic adrenocortical carcinoma. J Clin Endocrinol Metab 2007 01;92(0021-972; 1):148-154.

136. Abiven G, Coste J, Groussin L, Anract P, Tissier F, Legmann P, et al. Clinical and biological features in the prognosis of adrenocortical cancer: poor outcome of cortisol-secreting tumors in a series of 202 consecutive patients. J Clin Endocrinol Metab 2006 07;91(0021-972; 7):2650-2655.

137. Ayala-Ramirez M, Jasim S, Feng L, Ejaz S, Deniz F, Busaidy N, et al. Adrenocortical carcinoma: clinical outcomes and prognosis of 330 patients at a tertiary care center. Eur J Endocrinol 2013 Oct 23;169(6):891-899.

138. Margonis GA, Kim Y, Tran TB, Postlewait LM, Maithel SK, Wang TS, et al. Outcomes after resection of cortisol-secreting adrenocortical carcinoma. The American Journal of Surgery 2016;211(6):11061113. 
139. Berruti A, Fassnacht M, Haak H, Else T, Baudin E, Sperone P, et al. Prognostic role of overt hypercortisolism in completely operated patients with adrenocortical cancer. Eur Urol 2014 Apr;65(4):832-838.

140. Volante M, Bollito E, Sperone P, Tavaglione V, Daffara F, Porpiglia F, et al. Clinicopathological study of a series of 92 adrenocortical carcinomas: from a proposal of simplified diagnostic algorithm to prognostic stratification. Histopathology 2009;55(5):535-543.

141. Stojadinovic A, Ghossein RA, Hoos A, Nissan A, Marshall D, Dudas M, et al. Adrenocortical carcinoma: clinical, morphologic, and molecular characterization. J Clin Oncol 2002 02/15;20(0732183; 4):941-950.

142. Beuschlein F, Weigel J, Saeger W, Kroiss M, Wild V, Daffara F, et al. Major prognostic role of Ki67 in localized adrenocortical carcinoma after complete resection. J Clin Endocrinol Metab 2015 Mar; 100(3):841-849.

143. Assie G, Letouze E, Fassnacht M, Jouinot A, Luscap W, Barreau O, et al. Integrated genomic characterization of adrenocortical carcinoma. Nat Genet 2014 Jun;46(6):607-612.

144. Assié G, Jouinot A, Fassnacht M, Libé R, Garinet S, Jacob L, et al. Value of Molecular Classification for Prognostic Assessment of Adrenocortical Carcinoma. JAMA oncology 2019;5(10):1440-1447.

145. Zheng S, Cherniack AD, Dewal N, Moffitt RA, Danilova L, Murray BA, et al. Comprehensive pangenomic characterization of adrenocortical carcinoma. Cancer cell 2016;29(5):723-736.

146. Barreau O, Assie G, Wilmot-Roussel H, Ragazzon B, Baudry C, Perlemoine K, et al. Identification of a CpG island methylator phenotype in adrenocortical carcinomas. J Clin Endocrinol Metab 2013 Jan;98(1):E174-84.

147. De Reynies A, Assie G, Rickman DS, Tissier F, Groussin L, Rene-Corail F, et al. Gene expression profiling reveals a new classification of adrenocortical tumors and identifies molecular predictors of malignancy and survival. J Clin Oncol 2009 03/01;27(1527-7755; 7):1108-1115.

148. Lippert J, Appenzeller S, Liang R, Sbiera S, Kircher S, Altieri B, et al. Targeted molecular analysis in adrenocortical carcinomas: a strategy toward improved personalized prognostication. The Journal of Clinical Endocrinology \& Metabolism 2018;103(12):4511-4523.

149. D'Avolio A, De Francia S, Basile V, Cusato J, De Martino F, Pirro E, et al. Influence of the CYP2B6 polymorphism on the pharmacokinetics of mitotane. Pharmacogenet Genomics 2013 Mar 20. 
Part I

Clinical prediction 


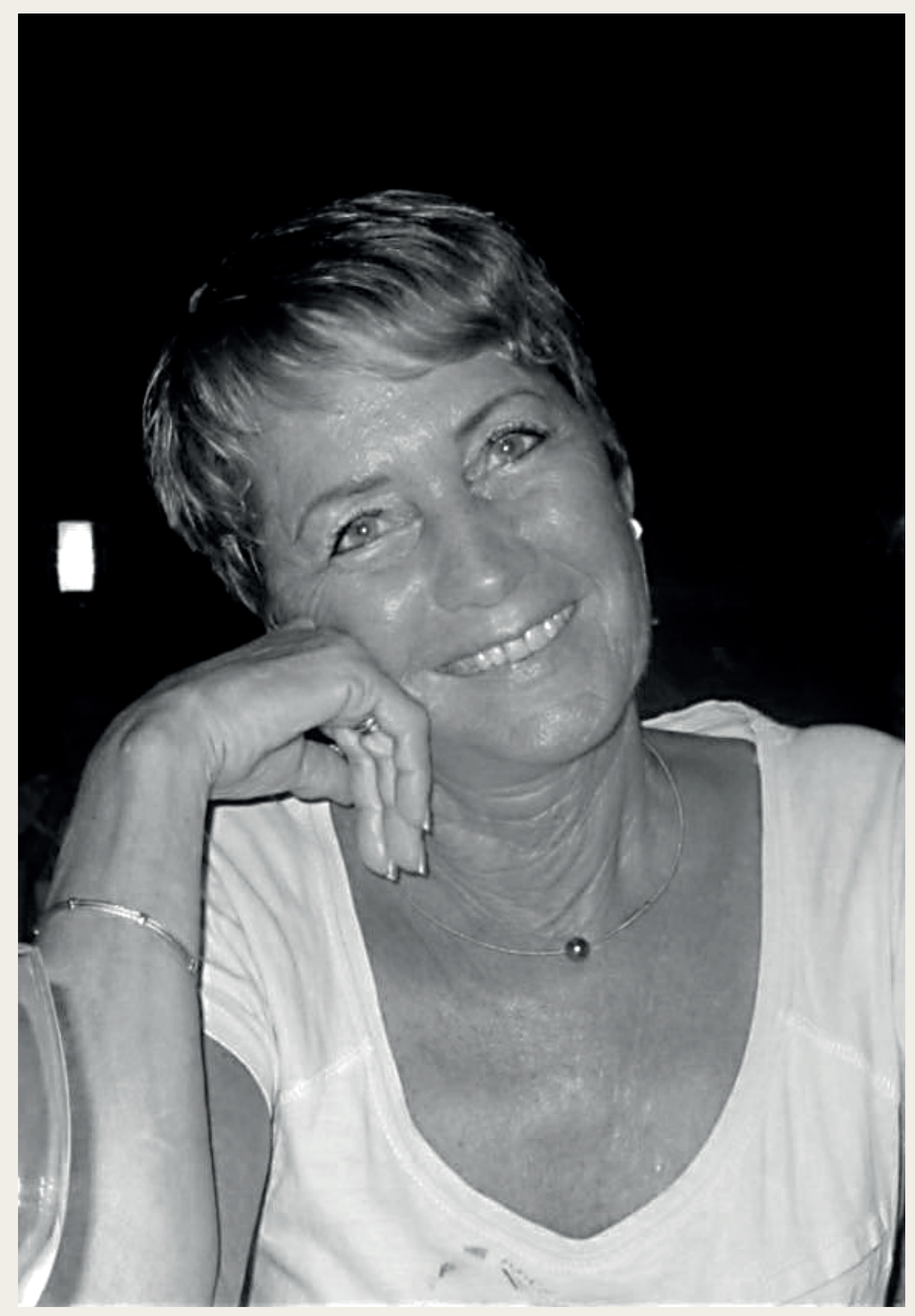




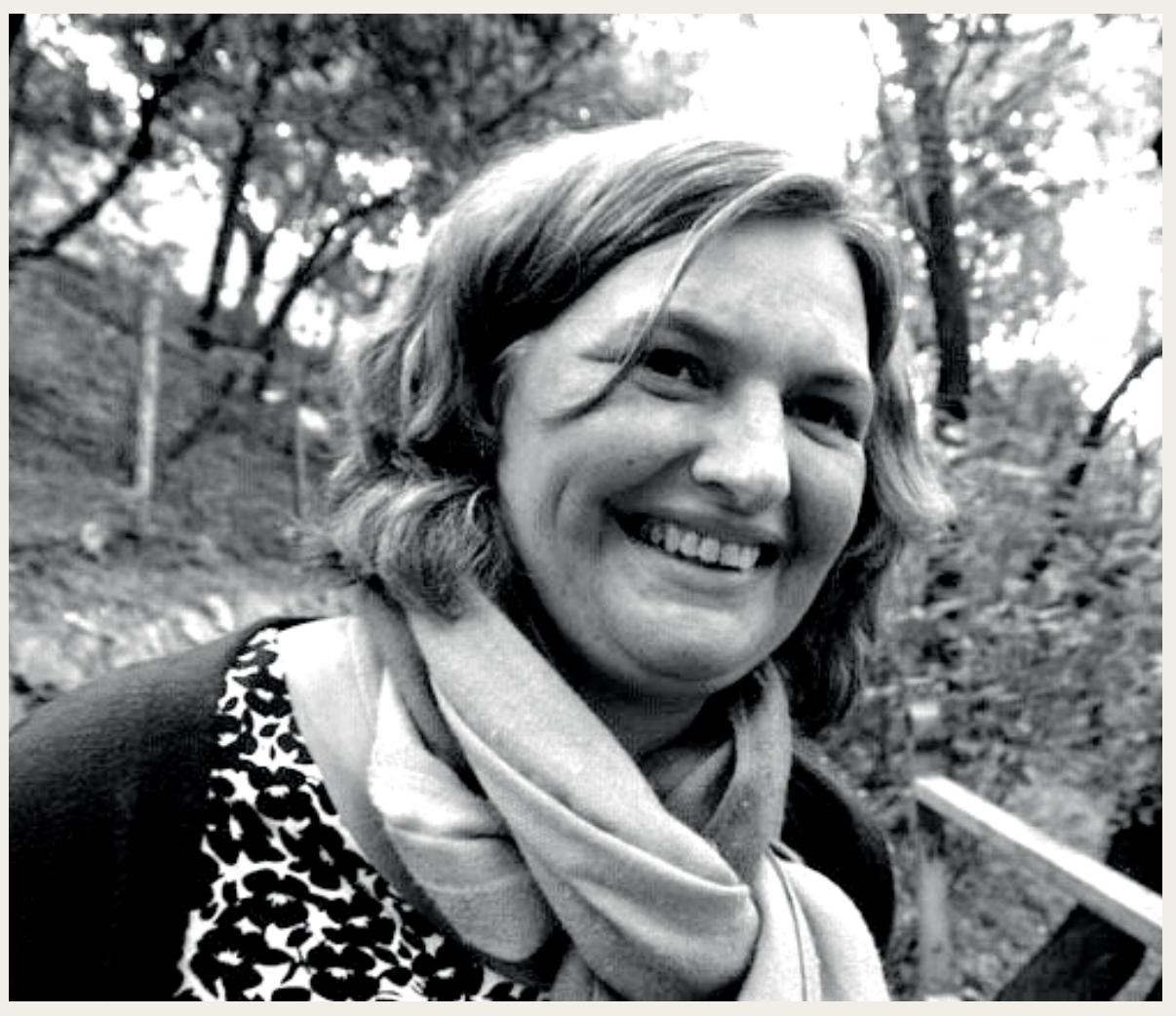




\section{Chapter 02}

Synchronous versus Metachronous Metastases in Adrenocortical

Carcinoma: an analysis of the Dutch Adrenal Network

Ettaieb MHT, Duker JC, Feelders RA, van der Kleij-Corssmit EPM, Menke-van der Houven van Oordt CW, Timmers HJLM, Kerstens MN, Wilmink JW, Zelissen PM, Havekes B, Haak HR

On behalf of the Dutch Adrenal Network

Hormones and Cancer, 2016 Dec;7(5-6):336-344. 


\section{Abstract}

Adrenal Cortical Carcinoma (ACC) is a rare malignancy with an incidence of 1.0 per million per year in the Netherlands. Median survival varies according to the European Network for the Study of Adrenal Tumours (ENS@T) tumour stage. It is unknown whether time until development of metastases is of influence on prognosis. To assess this, data were retrospectively obtained from centres of the Dutch Adrenal Network. Patients who presented with ACC between January 1, 2004 and October 31, 2013 were included. Date of detection of metastases, number of metastases and affected organs were registered. One hundred sixty patients were included in the analysis. Synchronous metastases were defined as diagnosis of metastasis $\leq 6$ months after the initial diagnosis of ACC. Overall survival rate was calculated from the date of diagnosis of metastasis until death from any cause.

At first presentation, 50 patients (31\%) had ACC with metastases (ENS@T stage IV). Another 67 (42\%) developed metastases during follow-up. Amongst the 117 patients with metastases, 84 (72 \%) patients had synchronous metastases and 33 (28\%) developed metachronous metastases. Diagnosis of synchronous metastases ( $p=$ 0.046), more than one affected organ $(p<0.001$ ) and four or more metastases ( $p<$ 0.001 ) were found to be associated with reduced overall survival. Limitations included retrospective design and limited details regarding pathological data.

We conclude that synchronous metastases of ACC are associated with a poorer prognosis compared to metachronous metastases of ACC. The clinical characteristics associated with prognosis in this study support the view to refine the prognostic classification for patients with stage IV ACC. 


\section{Introduction}

Adrenal Cortical Carcinoma (ACC) is a rare malignancy with an incidence of 1.0 per million in the Netherlands [1]. Median survival varies according to tumour stage, from 159 to 5months for patients with ENS@T stage I-II and ENS@T stage IV, respectively [1]. ACC usually presents with symptoms caused by hormone production or local symptoms due to tumour growth.

Radical surgical resection of locoregional tumour lesions is a mainstay of the curative treatment for ACC. Nevertheless, 75-85\% of patients who underwent radical resection showed recurrence of local disease often with concurrent distant metastases [2, 3]. Options for palliative treatment are limited.

Approximately, $30 \%$ of patients already present with metastases at the time of diagnosis [4]. The most common sites for metastases are the lungs ( 40\%), liver ( 40\%), skeleton ( 30\%) and lymph nodes ( 25\%) [5-7].

For various malignancies, it has been described that the distinction between synchronous and metachronous metastases has a prognostic value $[8,9]$. It is currently unknown, however, whether these different time patterns of metastases are associated with the outcome of patients with ACC. In addition, the influence of the number of metastases and affected organs on prognosis in patients with ACC is not well known. The number of involved organs in patients with stage IVACC has been suggested to be useful for the prediction of outcome [10].

The primary objective of the present retrospective study was to investigate if there was a difference in survival of ACC between patients with synchronous metastases and patients with metachronous metastases. In addition, we aimed to study the effect of the number of metastases and affected organs on survival.

\section{Methods}

Data were retrospectively obtained from the nine centres of the Dutch Adrenal Network (DAN). The DAN includes eight Dutch university hospitals (Erasmus Medical Centre Rotterdam, Leiden University Medical Centre, VU Medical Centre Amsterdam, Radboud University Medical Centre Nijmegen, University Medical Centre Groningen, Academic Medical Centre Amsterdam, University Medical Centre Utrecht and Maastricht University Medical Centre) and Máxima Medical Centre (MMC). It has initiated the registration of clinical data on ACC in a national database, e.g. for research purposes and the improvement of patient care [11,12].

Patients who presented with ACC between January 1, 2004 and October 31, 2013 were included. Start and end of the observation period coincided with the founding 
year of the DAN and the latest update of the DAN database, respectively.

In order to make the inclusion as complete as possible, hospital pathology departments, whom all participate in a nationwide network system (PALGA), were asked to compose a lists of all patients diagnosed with any adrenal tumour. Furthermore, the Dutch hospital diagnosis registry system, the Diagnosis Treatment Combination (DTC) system, was screened for patients labelled with an adrenal disease DTC code.

Inclusion criteria were age $\geq 18$ years and confirmed diagnosis of ACC, either histological (Weiss, Van Slooten, Ki67) or by a combination of clinical analysis, hormonal analysis (e.g. urinary steroid profiling, dexamethasone suppression test) and imaging data e.g. computed tomography (CT)

Patient records were assessed to obtain retrospective information on patient characteristics, metastases, radiology imaging (CT, Magnetic Resonance Imaging and positron emission tomography and treatment). Comorbidity was classified according to the Charlson Comorbidity Index (CCI) $[13,14]$.

\section{Definitions}

The primary endpoint was overall survival (OS). OS was calculated from the date of first diagnosis of metastases to death from any cause. One-year survival and 2-year survival were also calculated from the date of diagnosis of metastases. Conditional survival derived from the concept of conditional probability was calculated using the traditional Kaplan-Meier.

The mathematical definition used can be expressed as follows [15-18]:

$$
C S(t \mid s)=\frac{S(s+t)}{S(s)}
$$

Metastases were defined as synchronous when found $<6$ months after the initial diagnosis of ACC $[8,9,19]$.

Consequently, 'metachronous metastases' was defined as metastases diagnosed $\geq 6$ months after the initial diagnosis of ACC.

The number of metastases was classified as 'limited' if there were one to three metastases and as 'extended' if there were four or more metastases [20]. A distinction was made between metastases found in only one organ/lymph node or in more than one organ/lymph node localization (discriminated organs: liver, lung, bone, lymph nodes under diaphragm, lymph nodes above diaphragm and elsewhere). Multiple metastases found in one organ were registered as one affected organ in the analysis of number of affected organs. 
Diagnostic delay was calculated from the first hospital visit for ACC-related symptoms or signs until the moment the diagnosis of ACC were confirmed.

The effect of resection of the primary tumour on overall survival was assessed amongst patients with synchronous metastases. In addition, to further investigate the impact of ENS@Tstage at primary diagnosis, its impact on survival was analysed within the group of synchronous metastases. The IBM SPSS Statistics 22 was used for analysis. $T$ tests and Pearson chi-square tests were used to analyse patient characteristics. Univariate Cox regression was used to analyse the effect of synchronous metastases vs. metachronous metastases, one or more involved organs, number of metastases and different involved organs. Those factors found to be significant were analysed in a multivariate Cox-regression analysis. Cox regression was used to evaluate the effect of different treatments on 1-year survival and 2-year survival as well as overall survival. Survival was calculated using the Kaplan-Meier method and applied log-rank test to assess differences in survival rates.

\section{Results}

One hundred ninety-two patients were identified with adrenal tumour disease upon screening of provided PALGA and DTC patient lists. Adrenal metastases of other primary malignant origin, adenomas and pheochromocytomas were excluded at first screening. Finally, a total of 160 patients from the DAN centres fulfilled the inclusion criteria. Thirty-two patients were excluded: 30 patients were not diagnosed in the time period set for this study, 1 patient had an ectopic ACC and 1 patient's file was incomplete and data not retrievable.

Patient characteristics are summarised in Table 1. The ratio female to male patients was 1.6:1. Most patients in this study had stage II disease at diagnosis (36 \%) followed by stage IV disease (31\%). In 153 patients, ACC was confirmed based on histology of biopsy or tumour resection material. There were 7 patients in whom the diagnosis was solely based on the combination of clinical presentation, hormonal and imaging analysis and therefore treated as ACC (patients were not operated nor was there a biopsy performed). Median duration of follow-up was 17 months (range 0-118 months). Three patients in remission were lost to follow-up because they were referred back to their treating physician in a non-DAN hospital, and follow-up data were not available. During follow-up,

87 patients died as a direct result of ACC, 4 as a result of treatment toxicity and 2 patients died due to other causes ( 1 patient died of a pulmonary infection and the other patient died as a result of melanoma). At the end of follow-up, 23 patients were alive with disease and 44 patients were alive without evidence of disease. 
Table 1: Clinical characteristics of study population

\begin{tabular}{ll}
\hline Sex & N (\% of total 160) \\
\hline Male & $61(38 \%)$ \\
Female & $99(62 \%)$
\end{tabular}

Age (years)

Median

55 (range: 19-89)

\section{Follow up (months)}

Median

17(range: 0-118)

\section{Survival (months)}

Median

31 (range 0-118)

\section{ENS@T Stage at Diagnosis}

Survival

Median

Unknown

$2(1 \%)$

$9(6 \%)$

$58(36 \%)$

$41(26 \%)$

$50(31 \%)$

IV

\section{Metastases}

None

$43(27 \%)$

Synchronous metastases

$84(53 \%)$

Metachronous metastases

$33(21 \%)$

At the time of first metastases

Any time during follow up

Liver

$43(27 \%)$

$65(41 \%)$

Lung

$58(36 \%)$

79 (49\%)

Bone

$8(5 \%)$

$21(13 \%)$

Lymph nodes under

diaphragm

Lymph nodes above

diaphragm

Other location

$32(20 \%)$

$52(33 \%)$

$11(7 \%)$

$27(17 \%)$

$16(10 \%)$
62 months

32 months

7 months

* Could not be calculated 


\section{Survival}

Of the 160 included patients, 117 (73\%) were diagnosed with metastases during the follow-up period of this study: 84 patients had synchronous metastases and 33 developed metachronous metastases. Figure 1 shows the Kaplan-Meier curve of the time interval between initial diagnosis and detection of the first metastasis.

At 2-year survival, 28 (33\%) of the patients with synchronous metastases and 19 (58 $\%)$ of the patients with metachronous metastases were still alive $(p=0.036)$.

At the end of follow-up of this study, 16 patients (19\%) with synchronous and 13 patients (39\%) with metachronous metastases were still alive. Median overall survival after diagnosis of the first metastases for patients with synchronous or metachronous metastases was 12 and 29 months, respectively ( $p=0.046$; Fig. 2). The 1-year conditional survival for ACC patients with synchronous and metachronous metastases is presented in figure 3 .

Upon first diagnosis of metastatic disease, 40 patients (34 \%) had one to three metastases. The other 77 patients (66 \%) had four or more metastases. Median survival was 44 months for patients with 1-3 metastases and 7 months for patients with $\geq 4$ metastases $(p<0.001)$. Overall survival for these two groups was significantly different with 50 and $12 \%$, respectively $(p<0.001)$. Also, the number of affected organs was of influence on median survival. In 75 patients with only one affected organ, median survival was 24 months; in 42 patients with two or more involved organs, median survival was 4 months. Overall survival percentages were 33 and $10 \%$, respectively ( $p<0.001$ ) (Fig. 2 ). When analysing overall survival in a selected group of patients with $\geq 2$ affected organs $(n=42)$ by the factor synchronous vs. metachronous metastases, a log-rank $p$ value of 0.006 was found.

The results of synchronous disease, $\geq 2$ affected organs and $\geq 4$ metastases on 1-, 2 -year and overall survival in univariate and multivariate analysis are shown in Table 2. 


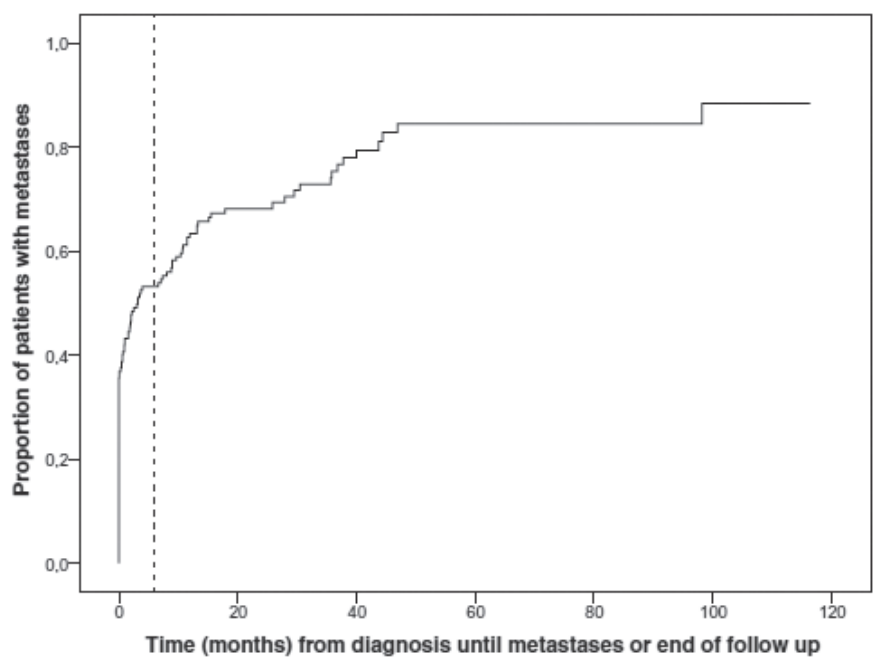

Fig. 1 Kaplan-Meier curve of the time in days between diagnosis of adrenocortical carcinoma and the diagnosis of the first metastases ( $n=160$ ). The cutoff ( 6 months) between synchronous and metachronous metastases is marked by the dotted line. 

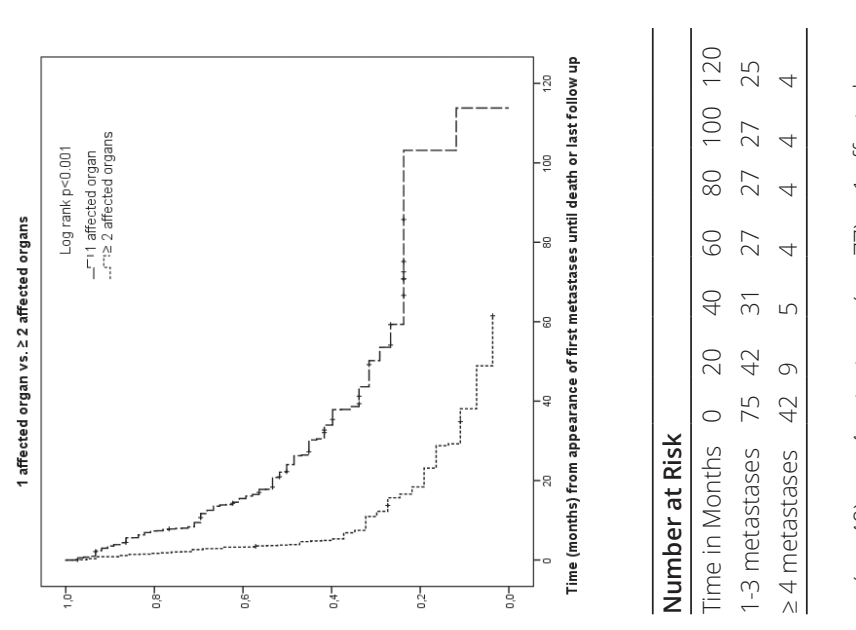

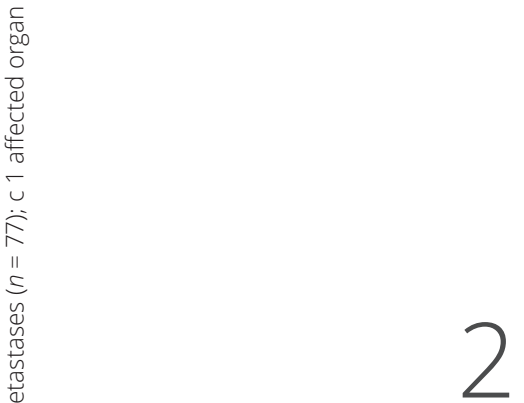
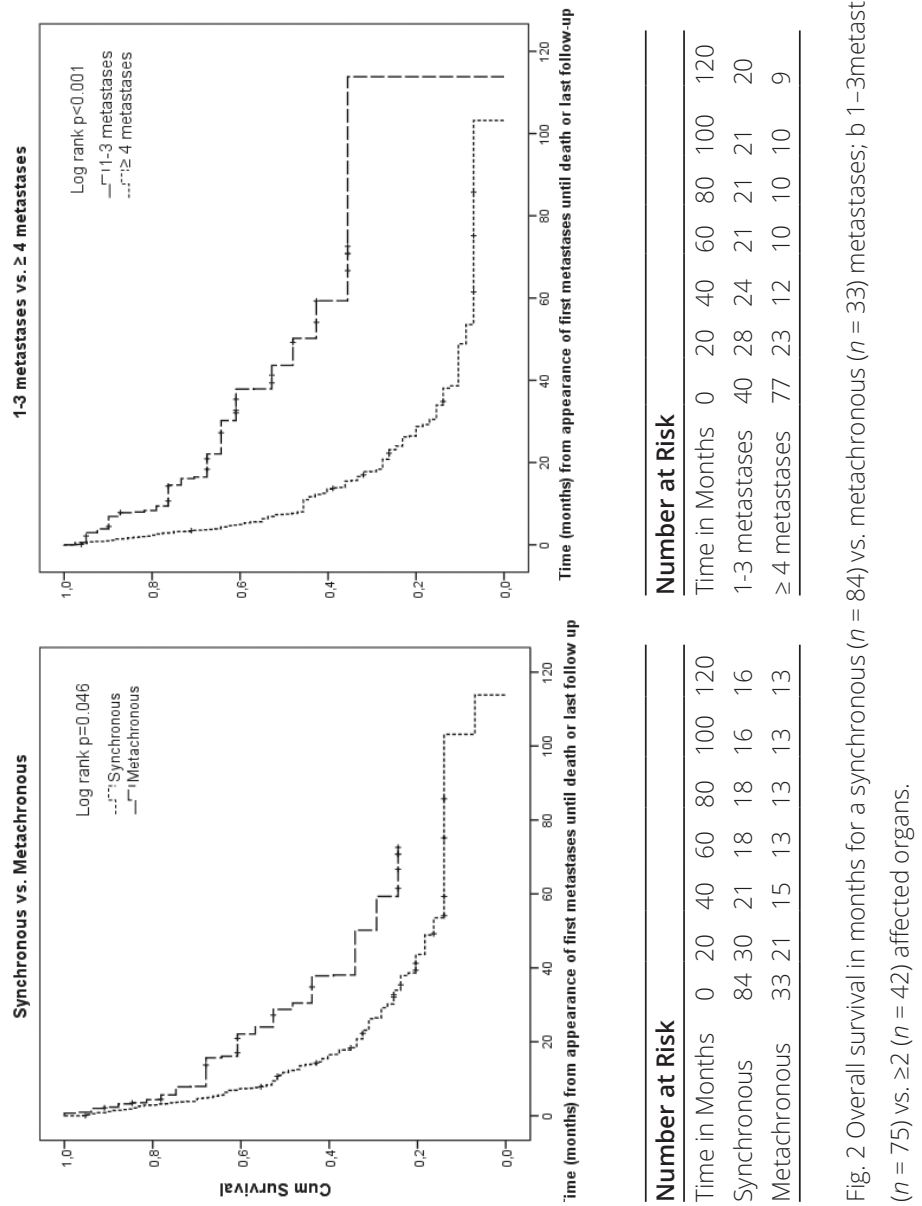


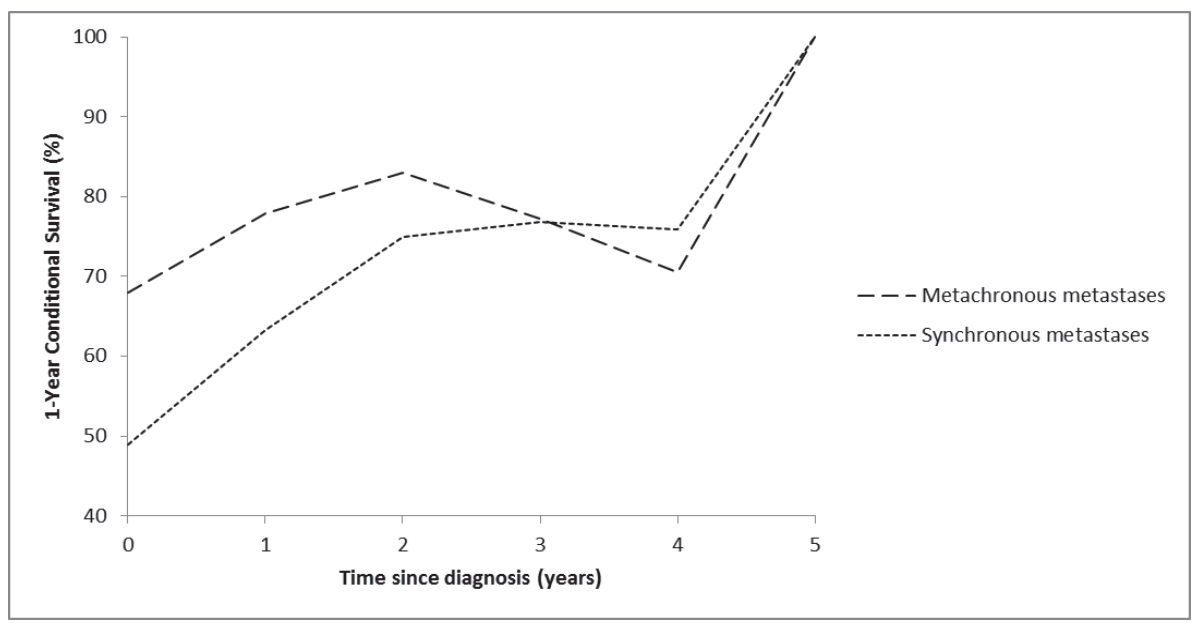

Fig. 3 One-year conditional survival CS (1 |s) in ACC patients with synchronous ( $N=84)$ and metachronous ( $N$ = 33) metastases.

\section{Affected Organs and Therapy}

Overall survival between subgroups with only one affected organ system (i.e., metastasis only in the lung and liver of lymph nodes) was not different.

Of the patients with only lung metastases $(n=27), 44 \%$ received chemotherapy (50\% EDP, $42 \%$ Streptozotocin, $8 \%$ Cisplatin), $17 \%$ had a metastasectomy and $89 \%$ received mitotane. For the patients with only liver metastases ( $n=20), 45 \%$ received chemotherapy (78 \% EDP, $11 \%$ Streptozotocin, $11 \%$ Cisplatin), $45 \%$ had a metastasectomy, $30 \%$ received radiofrequency ablation (RFA) and $75 \%$ received mitotane. In the group of patients with only lymph nodes under the diaphragm $(n=14), 36 \%$ received chemotherapy (80 \% EDP, $20 \%$ Streptozotocin), 57 \% had a metastasectomy and $79 \%$ were treated with mitotane.

One-year, 2-year and overall survival was not different between patients who received chemotherapy and those who did not in patients with only liver metastases ( $\mathrm{n}=$ 20), only lung metastases ( $n=27$ ) or only metastases in the lymph nodes under the diaphragm $(n=14)$. The small number of patients treated with radiotherapy precludes separate analysis of its potential benefit. Mitotane had a significant impact on one-year ( $p=0.017$ ), two-year ( $p=0.007$ ) and overall survival ( $p=0.007$ ) in the group of patients with only metastases in the lymph nodes under the diaphragm in comparison to those who did not receive mitotane in this group. In other subgroup analysis, mitotane showed no significant impact on survival in our population. 
RFA was performed in 6 patients with only liver metastases, and none of these patients died within 2 years $(p=0.046)$. Overall survival, however, of this subgroup was not different from the whole group of patients with liver metastases only ( $p=0.158)$.

Metastasectomy appeared to have a significant impact for patients with lung metastases only on overall survival $(p=0.012)$ in comparison to patients with lung metastases only who did not undergo a metastasectomy.

\section{Patients with Synchronous Metastases}

Amongst the patients with synchronous metastases of ACC $(n=84), 50$ patients (60 \%) were ENS@T stage IV ACC at primary diagnosis, 22 stage III (26 \%) and 12 stage II (14\%). Median time interval until diagnosis of metastasis was 0, mean time 17 days (range 0-119 days). Age and CCl did not differ significantly between patients who presented with stage IV at initial diagnosis or stage II-III. Patients with stage IV disease at initial diagnosis had significantly more affected organs compared to patients who developed metastases within 6 months after primary diagnosis ( $p=0.006$ ). Characteristics of these two subgroups are summarised in Table 3. Diagnostic delay (time in days until diagnosis of ACC was confirmed) did not differ significantly between these subgroups $(p=0.060)$.

The 1-year, 2-year and overall survival rates of patients who were stage IV at first presentation ( $n=50$ ) and of those who developed stage IV within 6 months after the initial primary diagnosis ( $n=34$ ) were significantly different (Table 3 ). Median survival was 7 and 23 months, respectively ( $p=0.003)$.

Sixty-seven of the synchronous metastasized patients did undergo an adrenalectomy for their primary tumour, whereas in 17 patients the primary tumour was not resected. All but 1 of the patients who did not undergo an adrenalectomy ( $n=17)$ had stage IV disease at the moment of diagnosis of ACC.

Patients with only one synchronous affected organ were more frequently submitted to a surgical resection of the primary adrenal tumour $(p=0.023)$. Of the 67 patients undergoing an adrenalectomy, 22 patients also had a metastasectomy. In contrast, of the 17 patients with synchronous metastases not undergoing an adrenalectomy, only 2 patients had a diagnostic metastasectomy to confirm ACC and determine the therapeutic strategy.

Median survival of patients with synchronous metastases who did or did not undergo adrenalectomy was 16.6 (range 0-113.8 months) and 1.8 months (range 0-16.5 months), respectively ( $<$ 0.001) (Fig. 4). This result did not change after correction for number of affected organs and $\mathrm{CCl}$. 
Chapter 2

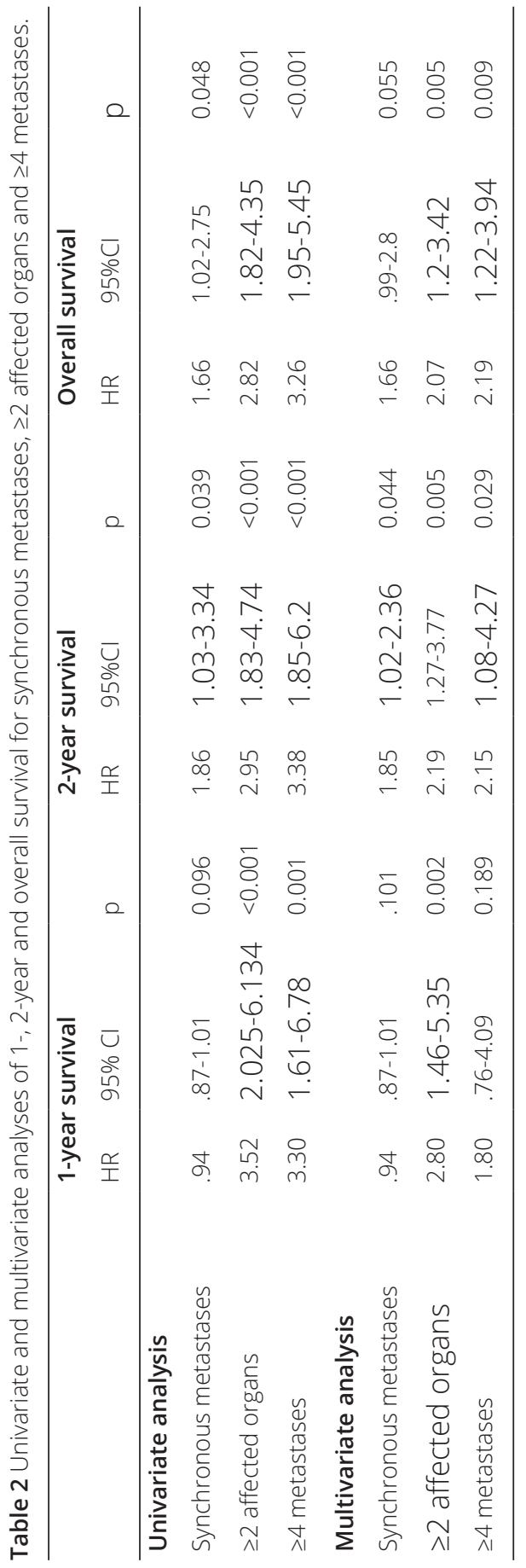


Table 3 Patient characteristics of patients with synchronous metastases: stage IV at diagnosis vs. metastases $\leq 6$ months but not at diagnosis

\begin{tabular}{|c|c|c|c|c|c|}
\hline & \multicolumn{2}{|c|}{ Stage IV at diagnosis $(n=50)$} & \multicolumn{3}{|c|}{$\begin{array}{l}\text { Metastases } \leq 6 \text { months, but not } \\
\text { at diagnosis }(n=34)\end{array}$} \\
\hline \multicolumn{6}{|l|}{$\operatorname{Sex}(n s)$} \\
\hline \multirow[t]{2}{*}{ Male } & 16 & $32 \%$ & Stage II & Stage III & $50 \%$ \\
\hline & & & 3 & 14 & \\
\hline Female & 34 & $68 \%$ & 9 & 8 & $40 \%$ \\
\hline \multicolumn{6}{|l|}{ Age (ns) } \\
\hline Mean & 53 & (range: 24-77) & 51 & & (range: 21-81) \\
\hline \multicolumn{6}{|c|}{ Charlson Comorbidity Index (ns) } \\
\hline Mean & 1.8 & & 1.4 & & \\
\hline \multicolumn{6}{|c|}{ Survival (months, $p=0.003$ ) } \\
\hline Median & 7 & (range: 0-114) & 23 & & (range: 0-103) \\
\hline \multicolumn{6}{|c|}{ Number of metastatic organs $(p=0.006)$} \\
\hline 1 & 25 & $50 \%$ & 27 & & $79 \%$ \\
\hline$\geq 2$ & 25 & $50 \%$ & 7 & & $21 \%$ \\
\hline \multicolumn{6}{|c|}{ Number of metastases (ns) } \\
\hline 1-3 metastases & 14 & $28 \%$ & 11 & & $33 \%$ \\
\hline $4+$ metastases & 36 & $72 \%$ & 23 & & $67 \%$ \\
\hline \multicolumn{6}{|c|}{ Adrenalectomy $(p=0.001)$} \\
\hline Yes & 34 & $68 \%$ & 33 & & $97 \%$ \\
\hline No & 16 & $32 \%$ & 1 & & $3 \%$ \\
\hline \multicolumn{6}{|c|}{ Diagnostic delay (months, ns) } \\
\hline Median & 1,3 & (range 0-11) & 2,1 & & Range (0-33) \\
\hline
\end{tabular}

Significant differences between the two groups are indicated with a $p$ value ns not significant 

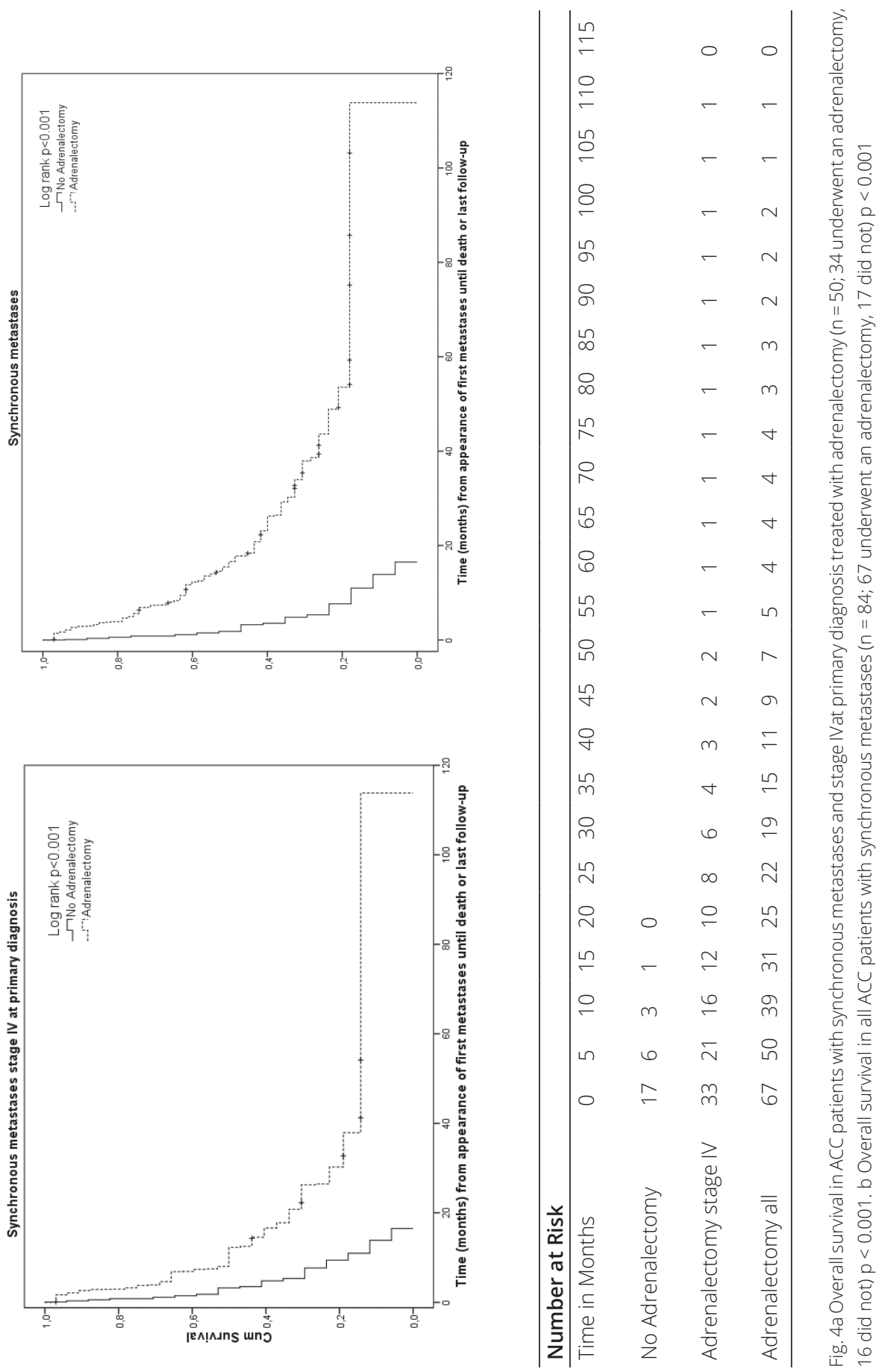


\section{Discussion}

In different types of cancer, the moment metastases occur has been shown to have a significant impact on survival. Synchronous metastases, defined as metastases originating within 6 months after diagnosis of the primary tumour, are associated with a significant negative effect on prognosis in the colon [21] and lung cancer [22].

A trend towards better survival in ACC patients with metachronous metastases compared to synchronous metastases has previously been described [10]. To our knowledge, this is the first study to describe that timing of metastases is associated with overall survival in ACC. Late synchronous metastases, i.e. development of stage IV disease within 6 months after stage II-III at primary diagnosis, were associated with improved survival compared to patients that present with stage IV disease.

The number of affected organs has been considered a prognostic factor for survival in metastatic ACC $[10,23]$. Our results confirm that the number of affected organs is indeed a prognostic factor.

Earlier studies evaluating the relationship between the number of metastatic lesions and survival have been inconclusive: In a cohort of 124 patients with ACC, Asssie et al. identified the presence of more than 5 metastatic lesions or the involvement of more than two organs as predictive factors for survival [10]. In the study by den Winkel et al., number of metastases, synchronous vs. metachronous metastatic disease and lymph node involvement did not significantly influence survival in a small cohort of 24 patients [24]. Our study shows a significant negative effect of having $\geq 4$ metastases on survival, suggesting that an increasing number of metastases is prognostically unfavourable. Moreover, our data suggest that adrenal cancer spread to the lymph nodes affects survival to the same extent as hematogenic metastases. These results support a modified ENS@T classification observed heterogeneity in survival of patients with stage IVACC [25].

Although it has been suggested that oligometastatic disease is associated with more favourable behaviour than extended metastatic disease, this has not resulted in a tailored treatment approach in ACC [26]. The low efficacy of current treatment options for ACC [27] raises the question whether patients with limited metastatic disease might benefit from a different approach.

Recent research shows that a radical adrenalectomy and metastasectomy in patients with synchronous, limited metastatic disease, resulted in a more favourable prognosis [28]. We found that a metastasectomy for ACC patients with lung metastases was associated with significant better overall survival. It has been suggested that patients with locally recurrent ACC also benefit from surgical treatment, but we did not examine the impact of local adrenal recurrence on prognosis [29]. Previous studies demonstrated a better prognosis in patients with a time to local recurrence longer 
than 1 year, and those in whom a complete resection of the local recurrence was performed [30].

In this study, adrenalectomy had a strong influence on survival, even in the presence of metastases. Patients with only one synchronous affected organ more frequently underwent an adrenalectomy than patients with two or more affected organs. Of notice, we were unable to find any other difference in clinical characteristics between patients who did undergo an adrenalectomy and those who did not. We had expected that patients with a bad performance status at disease presentation would have been less likely to have undergone surgical resection.

The improved survival that is associated with surgical intervention [31, 32] might overrule decision-making based on performance status and stage of disease in this group to a certain extent.

We found that patients who presented with stage IV disease at diagnosis had a much shorter survival than patients with stage II-III disease at diagnosis who developed late synchronous metastases. Thus, even within the group of synchronous metastases, a difference in survival was observed between early and late metastases. Future analysis should focus on identifying this specific subgroup of patients with ACC which exhibits less aggressive behaviour, who may benefit from adrenalectomy[33].

Our study is limited because of its retrospective nature. There were missing data, especially pathological data. The present study, however, was performed in a relatively large and well-defined cohort of adrenocortical cancer patients that reliably reflects the Dutch ACC population as a whole [1].

Current treatment options in ACC are limited, and new therapeutic options are anticipated[34]. Metachronous metastases are associated with better overall survival. Local therapy directed at metastasis should be considered in patients with limited metachronous disease. Otherwise, mitotane in combination with chemotherapy according to the FIRM-ACT protocol is indicated in case of more extended metachronous or synchronous metastases [34], although the latter patient group often has a bad prognosis despite systemic therapy [27]. The role of other local therapies already implemented in other malignancies has not been thoroughly investigated in ACC.

Stereotactic radiotherapy for lung metastases could be a promising alternative to surgical intervention. Radiofrequency ablation (RFA) for liver metastases, as a localregional treatment with relatively little morbidity, should be further investigated as a therapeutic option in the treatment of selected cases of liver metastases in ACC.

In conclusion, synchronous metastases of ACC are associated with a worse prognosis compared to metachronous metastases of ACC and patients with late synchronous 
metastases have a better prognosis than those with metastases at initial diagnosis. Further refinement of ENSAT stage IV disease should be considered, as these prognostic differences could be taken into account when determining the optimal treatment for a patient with ACC. Number of affected organs, number of metastases and timing of development of metastases influence survival and could potentially be considered in therapeutic decision making. 


\section{References}

1. Kerkhofs TM, Verhoeven RH, Van der Zwan JM, Dieleman J, Kerstens MN, Links TP, Van de PollFranse LV, Haak HR 2013 Adrenocortical carcinoma: A population-based study on incidence and survival in the netherlands since 1993. Eur J Cancer 49(11):2579-2586

2. Pommier RF, Brennan MF 1992 An eleven-year experience with adrenocortical carcinoma. SURGERY-SAINT LOUIS- 112:963-963

3. Terzolo M, Angeli A, Fassnacht M, Daffara F, Tauchmanova L, Conton PA, Rossetto R, Buci L, Sperone P, Grossrubatscher E, Reimondo G, Bollito E, Papotti M, Saeger W, Hahner S, Koschker AC, Arvat E, Ambrosi B, Loli P, Lombardi G, Mannelli M, Bruzzi P, Mantero F, Allolio B, Dogliotti L, Berruti A 2007 Adjuvant mitotane treatment for adrenocortical carcinoma. N Engl J Med 356(1533-4406; 23):2372-2380

4. Koschker AC, Fassnacht M, Hahner S, Weismann D, Allolio B 2006 Adrenocortical carcinoma -. improving patient care by establishing new structures. Exp Clin Endocrinol Diabetes 114(09477349; 0947-7349; 2):45-51

5. Fassnacht M, Allolio B 2009 Clinical management of adrenocortical carcinoma. Best Pract Res Clin Endocrinol Metab 23(1532-1908; 2):273-289

6. Lafemina J, Brennan MF 2012 Adrenocortical carcinoma: Past, present, and future. J Surg Oncol 106(5):586-594

7. Glover AR, Ip JC, Zhao JT, Soon PS, Robinson BG, Sidhu SB 2013 Current management options for recurrent adrenocortical carcinoma. Onco Targets Ther 6:635-643

8. Mekenkamp LJ, Koopman M, Teerenstra S, van Krieken JH, Mol L, Nagtegaal ID, Punt CJ 2010 Clinicopathological features and outcome in advanced colorectal cancer patients with synchronous vs metachronous metastases. Br J Cancer 103(2):159-164

9. Howell GM, Carty SE, Armstrong MJ, Stang MT, McCoy KL, Bartlett DL, Yip L 2013 Outcome and prognostic factors after adrenalectomy for patients with distant adrenal metastasis. Ann Surg Oncol 20(11):3491-3496

10. Assie G, Antoni G, Tissier F, Caillou B, Abiven G, Gicquel C, Leboulleux S, Travagli JP, Dromain C, Bertagna X, Bertherat J, Schlumberger M, Baudin E 2007 Prognostic parameters of metastatic adrenocortical carcinoma. J Clin Endocrinol Metab 92(0021-972; 1):148-154

11. Hermsen IG, Kerkhofs TM, Butter G, Kievit J, van Eijck CH, Nieveen van Dijkum EJ, Haak HR, Dutch Adrenal Network 2012 Surgery in adrenocortical carcinoma: Importance of national cooperation and centralized surgery. Surgery 152(1):50-56

12. Kerkhofs TM, Verhoeven RH, Bonjer HJ, van Dijkum EJ, Vriens MR, De Vries J, Van Eijck CH, Bonsing BA, Van de Poll-Franse LV, Haak HR, Dutch Adrenal Network 2013 Surgery for adrenocortical carcinoma in the netherlands: Analysis of the national cancer registry data. Eur J Endocrinol 169(1):83-89

13. Charlson ME, Pompei P, Ales KL, MacKenzie CR 1987 A new method of classifying prognostic comorbidity in longitudinal studies: Development and validation. J Chronic Dis 40(5):373-383

14. Charlson M, Szatrowski TP, Peterson J, Gold J 1994 Validation of a combined comorbidity index. J Clin Epidemiol 47(11):1245-1251

15. Hieke S, Kleber M, Konig C, Engelhardt M, Schumacher M 2015 Conditional survival: A useful concept to provide information on how prognosis evolves over time. Clin Cancer Res 21(7):15301536

16. Zamboni BA, Yothers G, Choi M, Fuller CD, Dignam J, Raich PC, Thomas CR,Jr, O'Connell MJ, Wolmark N, Wang SJ 2010 Conditional survival and the choice of conditioning set for patients 
with colon cancer: An analysis of NSABP trials C-03 through C-07. J Clin Oncol 28(15):2544-2548

17. Zabor EC, Gonen M, Chapman PB, Panageas KS 2013 Dynamic prognostication using conditional survival estimates. Cancer 119(20):3589-3592

18. van Houwelingen $\mathrm{H}$, Putter $\mathrm{H} 2011$ Dynamic prediction in clinical survival analysis. CRC Press

19. Turanli S 2012 Importance of the development time of isolated bone metastasis in breast cancer. Langenbecks Arch Surg 397(6):967-972

20. Weichselbaum RR, Hellman S 2011 Oligometastases revisited. Nat Rev Clin Oncol 8(6):378-382

21. Kumar R, Price TJ, Beeke C, Jain K, Patel G, Padbury R, Young GP, Roder D, Townsend A, Bishnoi S, Karapetis CS 2014 Colorectal cancer survival: An analysis of patients with metastatic disease synchronous and metachronous with the primary tumor. Clin Colorectal Cancer 13(2):87-93

22. Tanvetyanon T, Robinson LA, Schell MJ, Strong VE, Kapoor R, Coit DG, Bepler G 2008 Outcomes of adrenalectomy for isolated synchronous versus metachronous adrenal metastases in non-smallcell lung cancer: A systematic review and pooled analysis. J Clin Oncol 26(7):1142-1147

23. Malandrino P, Al Ghuzlan A, Castaing M, Young J, Caillou B, Travagli JP, Elias D, de Baere T, Dromain C, Paci A, Chanson P, Schlumberger M, Leboulleux S, Baudin E 2010 Prognostic markers of survival after combined mitotane- and platinum-based chemotherapy in metastatic adrenocortical carcinoma. Endocr Relat Cancer 17(3):797-807

24. op den Winkel J, Pfannschmidt J, Muley T, Grunewald C, Dienemann H, Fassnacht M, Allolio B 2011 Metastatic adrenocortical carcinoma: Results of 56 pulmonary metastasectomies in 24 patients. Ann Thorac Surg 92(6):1965-1970

25. Libe R, Borget I, Ronchi CL, Zaggia B, Kroiss M, Kerkhofs T, Bertherat J, Volante M, Quinkler M, Chabre O, Bala M, Tabarin A, Beuschlein F, Vezzosi D, Deutschbein T, Borson-Chazot F, Hermsen I, Stell A, Fottner C, Leboulleux S, Hahner S, Mannelli M, Berruti A, Haak H, Terzolo M, Fassnacht M, Baudin E, ENSAT network 2015 Prognostic factors in stage III-IV adrenocortical carcinomas (ACC): An european network for the study of adrenal tumor (ENSAT) study. Ann Oncol 26(10):2119-2125

26. Huang F, Wu G, Yang K 2014 Oligometastasis and oligo-recurrence: More than a mirage. Radiat Oncol 9:230-014-0230-6

27. Fassnacht M, Terzolo M, Allolio B, Baudin E, Haak H, Berruti A, Welin S, Schade-Brittinger C, Lacroix A, Jarzab B, Sorbye H, Torpy DJ, Stepan V, Schteingart DE, Arlt W, Kroiss M, Leboulleux S, Sperone P, Sundin A, Hermsen I, Hahner S, Willenberg HS, Tabarin A, Quinkler M, de IF, Schlumberger M, Mantero F, Weismann D, Beuschlein F, Gelderblom H, Wilmink H, Sender M, Edgerly M, Kenn W, Fojo T, Müller H, Skogseid B 2012 Combination chemotherapy in advanced adrenocortical carcinoma. N Engl J Med

28. Dy BM, Strajina V, Cayo AK, Richards ML, Farley DR, Grant CS, Harmsen WS, Evans DB, Grubbs EG, Bible KC, Young WF, Perrier ND, Que FG, Nagorney DM, Lee JE, Thompson GB 2014 Surgical resection of synchronously metastatic adrenocortical cancer. Ann Surg Oncol

29. Dy BM, Wise KB, Richards ML, Young WF,Jr, Grant CS, Bible KC, Rosedahl J, Harmsen WS, Farley DR, Thompson GB 2013 Operative intervention for recurrent adrenocortical cancer. Surgery 154(6):1292-9; discussion 1299

30. Erdogan I, Deutschbein T, Jurowich C, Kroiss M, Ronchi C, Quinkler M, Waldmann J, Willenberg HS, Beuschlein F, Fottner C, Klose S, Heidemeier A, Brix D, Fenske W, Hahner S, Reibetanz J, Allolio B, Fassnacht M, German Adrenocortical Carcinoma Study Group 2013 The role of surgery in the management of recurrent adrenocortical carcinoma. J Clin Endocrinol Metab 98(1):181-191

31. Livhits M, Li N, Yeh MW, Harari A 2014 Surgery is associated with improved survival for adrenocortical cancer, even in metastatic disease. Surgery 156(6):1531-40; discussion 1540-1

32. Dy BM, Strajina V, Cayo AK, Richards ML, Farley DR, Grant CS, Harmsen WS, Evans DB, Grubbs 
Chapter 2

EG, Bible KC, Young WF, Perrier ND, Que FG, Nagorney DM, Lee JE, Thompson GB 2015 Surgical resection of synchronously metastatic adrenocortical cancer. Ann Surg Oncol 22(1):146-151

33. Hermsen IG, Gelderblom H, Kievit J, Romijn JA, Haak HR 2008 Extremely long survival in six patients despite recurrent and metastatic adrenal carcinoma. Eur J Endocrinol 158(1479-683; 6):911-919

34. Kerkhofs TM, Ettaieb MH, Hermsen IG, Haak HR 2015 Developing treatment for adrenocortical carcinoma. Endocr Relat Cancer 22(6):R325-38 
2 


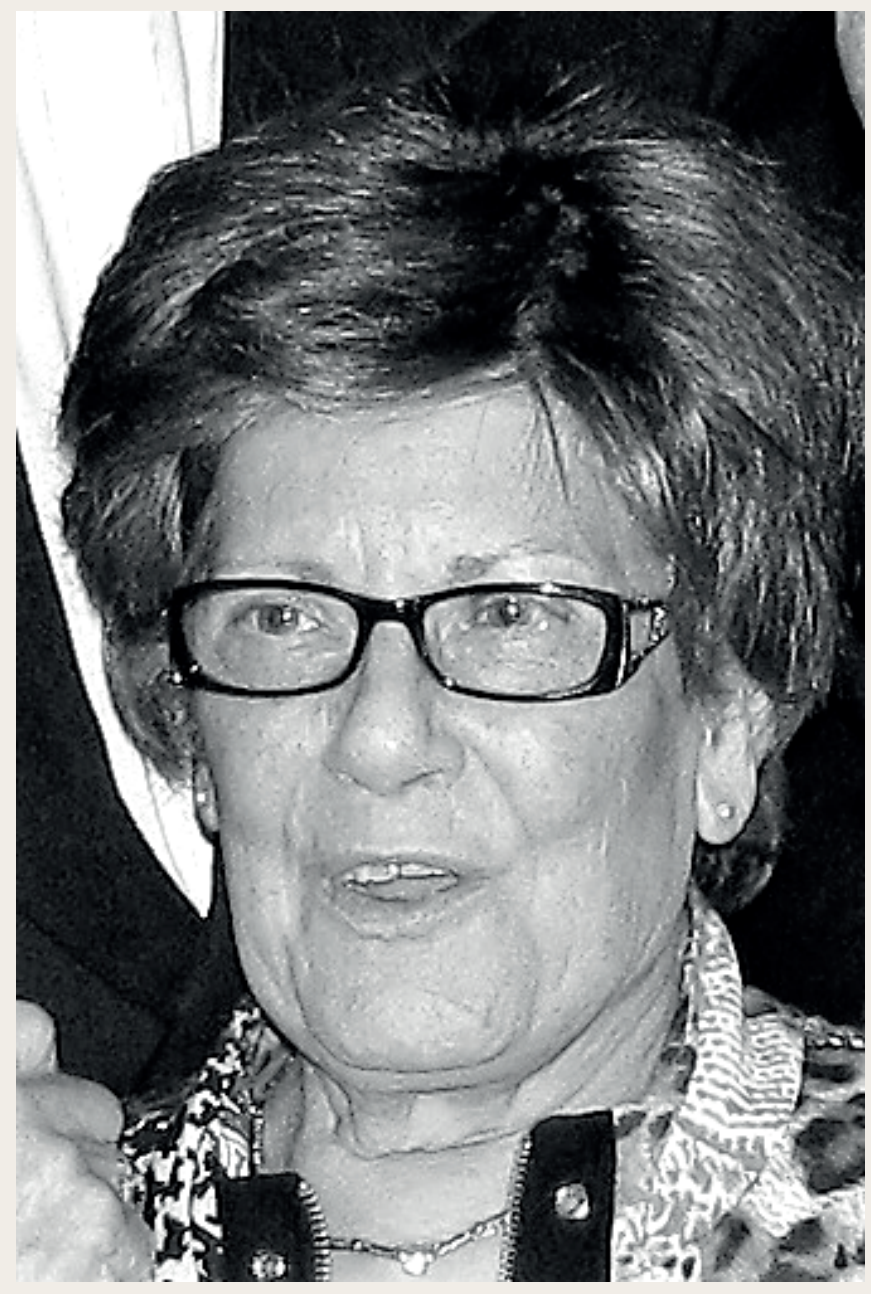




\section{Chapter 03}

Development and Internal Validation of a Multivariable Prediction Model for Adrenocortical-CarcinomaSpecific Mortality

Madeleine HT Ettaieb, Sander MJ van Kuijk, Annelies de Wit-Pastoors, Richard A Feelders, Eleonora PM Corssmit, Elisabeth MW Eekhoff, Paul van der Valk, Henri JLM Timmers, Michiel N Kerstens, Heinz-Josef Klümpen, Rachel S van Leeuwaarde, Bas Havekes and Harm R Haak

on behalf of the Dutch Adrenal Network

Cancers (Basel). 2020 Sep 22;12(9):E2720. 


\section{Simple Summary}

Adrenocortical carcinoma is a rare and aggressive cancer. Great variability in clinical course is observed, ranging from patients with extreme long survival to aggressive tumors with prompt fatal outcome. This heterogeneity in survival makes it complicated to tailor treatment strategies for an individual patient. Therefore we sought to identify prognostic factors associated with ACC specific mortality. We analyzed the data of 160 ACC patients and developed a clinical prediction model including age, modified European Network for the Study of Adrenal Tumors (mENSAT) stage, and radical resection. This easy-to-use prediction model for ACC-specific mortality has the potential to guide clinical decision making if externally validated.

\section{Abstract}

Adrenocortical carcinoma (ACC) has an incidence of about 1.0 per million per year. In general, survival of patients with ACC is limited. Predicting survival outcome at time of diagnosis is a clinical challenge. The aim of this study was to develop and internally validate a clinical prediction model for ACC-specific mortality. Data for this retrospective cohort study were obtained from the nine centers of the Dutch Adrenal Network (DAN). Patients who presented with ACC between 1 January 2004 and 31 October 2013 were included. We used multivariable Cox proportional hazards regression to compute the coefficients for the prediction model. Backward stepwise elimination was performed to derive a more parsimonious model. The performance of the initial prediction model was quantified by measures of model fit, discriminative ability, and calibration. We undertook an internal validation step to counteract the possible overfitting of our model. A total of 160 patients were included in the cohort. The median survival time was 35 months, and interquartile range (IQR) 50.7 months. The multivariable modeling yielded a prediction model that included age, modified European Network for the Study of Adrenal Tumors (mENSAT) stage, and radical resection. The c-statistic was 0.77 (95\% Confidence Interval: $0.72,0.81$ ), indicating good predictive performance. We developed a clinical prediction model for ACCspecific mortality. ACC mortality can be estimated using a relatively simple clinical prediction model with good discriminative ability and calibration. 


\section{Introduction}

Adrenocortical carcinoma (ACC) is a rare malignancy with an annual incidence of about 1.0 per million individuals [1]. With an overall one-year survival rate of $60 \%$ and a five-year survival rate of 32\% [1], the disease has a poor prognosis in general. Nonetheless, patients who survive for 12-28 years have been documented [2]. The exact reason for the long survival in these patients is unknown. Several factors may be responsible for these differences in survival, e.g., tumor type, lifestyle, and genetic variability. Predicting survival outcome at the time of diagnosis is difficult in clinical practice. Some clinical variables show prognostic potential: the Weiss score is the internationally acknowledged pathologic scoring system to differentiate between malignant and benign tumors. The Weiss score is a nine-point scoring system, in which a score of three or higher corresponds to a high probability of malignancy $[3,4]$. A Weiss score greater than six has been found to correspond to poor prognosis [5].

Another histological weighted scoring system is the Van Slooten Index (VSI). A score of eight or higher corresponds to a high probability of malignancy [6]. Unfortunately, neither of these pathological features are sufficiently specific for an estimation of prognosis in ACC.

The Ki67 index, an immunohistochemical marker, has been suggested as an additional prognostic parameter. The Ki67 index is an estimate of the percentage of tumor proliferation. A Ki67 index over 5\% is suggestive of malignancy [7]. A malignant tumor with a Ki67 index under 10\% is associated with a relatively good prognosis; an index over $20 \%$ is associated with an undesirable course of disease $[5,8]$. However, the Ki67 scoring assessment varies greatly, and both inter- and intra-observer variations cause significant limitations to its clinical utility [9]. Additionally, the clinically relevant cutoff values that are suggested to be associated with prognosis are debatable.

Other molecular or genomic prognostic markers that can clearly distinguish between low-risk and high-risk ACC tumors are being increasingly investigated [10], but their clinical value as prognostic tools has not yet been determined in large prospective series and therefore they are not part of the current clinical guidelines [11].

In 2004, the International Union Against Cancer (UICC) and the World Health Organization published the first staging classification based on the Tumor, Node, Metastasis (TNM) criteria for ACC [12]. Due to shortcomings, the European Network for the Study of Adrenal Tumors (ENSAT) developed a revised staging system [13]. The ENSAT staging system is currently recommended for estimating the prognosis of a patient with ACC $[5,12]$. However, the ENSAT stage is still associated with considerable heterogeneity, as reflected by a five-year stage-dependent survival of 66-82\% for stage I, 58-64\% for stage II, 24-50\% for stage III, and 0-17\% for stage IV [14]. A prediction model combining multiple parameters that have been individually associated with survival could be of 
significant additional value, and may be more useful in clinical practice. A clinical prediction model can inform patients and their physicians of the patients' probability of a specified outcome and help them with associated decision making. Previous prediction models were developed for specific subgroups of ACC patients: recurrence-free (RFS) and overall survival (OS) after curative resection of ACC [15], OS of ACC patients after surgery [16], or lacking essential (histologic) predictors in the model development [17-19]. Therefore, the aim of this study is to develop and internally validate a multivariable, generally applicable clinical prediction model for ACC-specific mortality.

\section{Results}

A total of 160 patients were included in the cohort. One patient was omitted from the analysis, as no outcome measures were available. Patients were followed for a median period of 33 months (1st and 3rd quartile: 11.0-61.5). A total of 108 (67.9\%) patients died during the course of follow-up. The median survival time was 35.6 months (range 0.7-145.4 months). The characteristics of all patients included in this study are shown in Table 1. Figure 1 shows the Kaplan-Meier curve of the total cohort, for a total follow-up time of 60 months.

Table 1. Patient characteristics $(N=160)$ *

\begin{tabular}{lll}
\hline & Original Data & Imputed Data \\
\hline Male & $\mathrm{N}(\%$ of total) (Range) & $\mathrm{N}(\%$ of total) (Range) \\
Female & $61(38)$ & $61(38)$ \\
Age at diagnosis & $99(62)$ & $99(62)$ \\
ENSAT Stage & $55(19-89)$ & $55(19-89)$ \\
I & & \\
II & $9(6)$ & $9(6)$ \\
III & $58(36)$ & $58(36)$ \\
IV & $42(26)$ & $42(26)$ \\
mENSAT Stage & $51(32)$ & $51(32)$ \\
I & & $9(6)$ \\
II & $9(6)$ & $58(36)$ \\
III & $58(36)$ & $30(19)$ \\
IVa & $30(19)$ & $36(22)$ \\
IVb & $36(22)$ & $17(11)$ \\
IVC & $17(11)$ & $10(6)$ \\
\hline
\end{tabular}




\begin{tabular}{lll}
\hline & Original Data & Imputed Data \\
\hline Resection status & $74(46,25)$ & $74(46)$ \\
R0 & $61(38,1)$ & $68(43)$ \\
R1/R2/Rx & $18(11,25)$ & $18(11)$ \\
No surgery & $7(4,4)$ & - \\
Missing & & \\
Ki67 & $27(17)$ & $47(29)$ \\
$\leq 5$ & $19(12)$ & $28(18)$ \\
$6-10$ & $8(5)$ & $16(10)$ \\
$11-15$ & $30(18,5)$ & $69(43)$ \\
$>15$ & $76(47,5)$ & - \\
Missing & & $125(78)$ \\
Capsular and/or vascular invasion & $90(56)$ & $35(22)$ \\
Yes & $24(15)$ & - \\
No & $46(29)$ & - \\
Missing & & $88(55)$ \\
Hypercortisolism & $88(55)$ & - \\
Yes & $35(22)$ & \\
No & $37(23)$ & - \\
Missing & 78 & \\
Complaints due to tumor mass & & \\
Yes & & \\
No & & \\
Missing & & \\
\hline
\end{tabular}

* To prevent a loss of statistical precision and to decrease the likelihood of obtaining biased results, we imputed the available data (see methods for further explanation). See method section for definition of the predictor variables. 


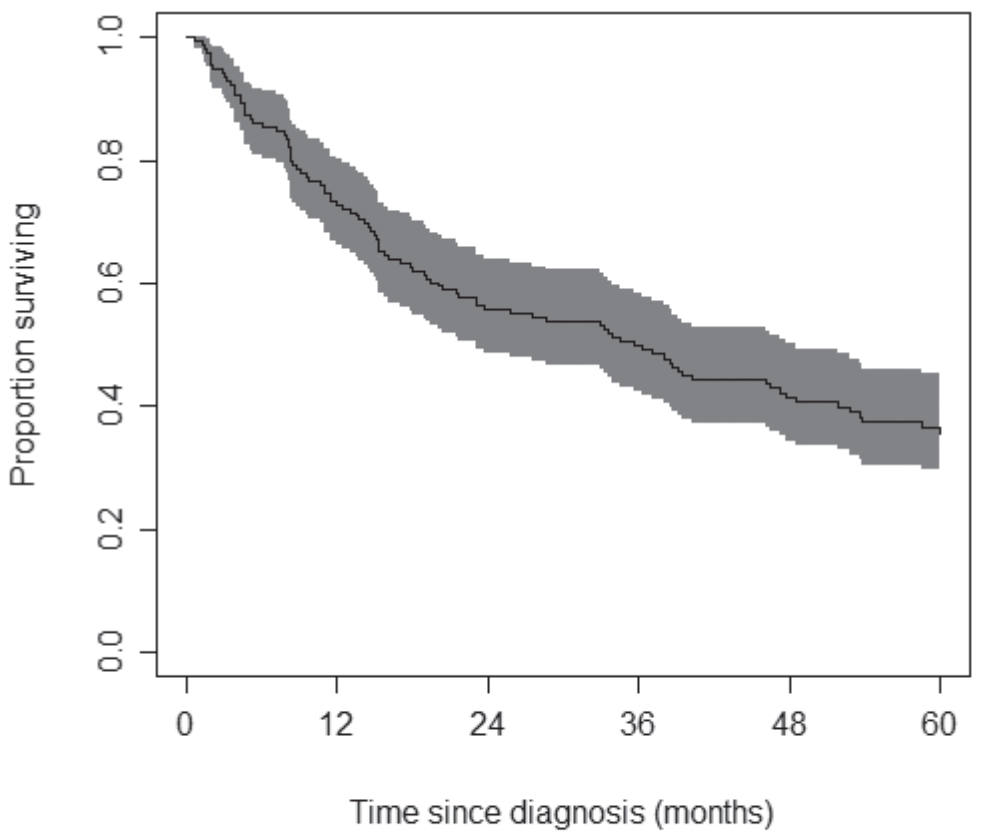

Number at risk

$\begin{array}{llllll}159 & 115 & 87 & 75 & 57 & 40\end{array}$

Figure 1. Kaplan-Meier curve, including 95\% confidence band of the risk of adrenocortical carcinoma-related mortality. The numbers above the $x$-axis denote the number of patients still at risk at each point in time.

In the original database, before attempted revision, 48 (30\%) out of 160 patients had a Ki67 score. Fifty-five of 160 (34\%) tumor samples were retrieved for central revision of the Ki67. Of only 49 samples, the appropriate material was available for immunohistochemistry analysis and of these 49 samples 13 tumors already had a Ki67 record in the database (net: $49-13=36$ new Ki67 data). Revision data were used for final analysis. The number of valid records increased from 48 (30\%) in the original database to $(48+36) 84(52.5 \%)$ in the new database.

The correlation between ENSAT and mENSAT was high ( $r h o=0.93, p<0.001$ ). We selected mENSAT for further statistical modelling based on a stronger univariable association with survival. The c-statistic for mENSAT was 0.73 , compared to 0.71 for ENSAT. The restricted cubic spline regression revealed no significant non-linear associations between continuous predictor variables and mortality. Because of the high proportion of missing values on the Ki67, the modelling procedure was performed both with and without Ki67. 
Table 2 shows the coefficients of the predictor variables that were significantly associated with ACC-related mortality. In the model that was derived without the Ki67 index, cortisol and pathology positivity were also excluded during the backward elimination process because their $p$-values were too high ( $p=0.469$ and $p=0.155$ upon exclusion, respectively). All predictors were risk factors for ACC-specific mortality, except for radical resection, which was protective. The scaled Schoenfeld residuals revealed that no predictor variables violated the proportional hazards assumption, indicating that the predictor variables are valid for the whole follow-up period.

Pathology was scored positive if venous invasion or capsular invasion were present according to the Weiss criteria in the pathology report or capsular and/or vascular invasion was scored yes according to the Van Slooten Index in the pathology report (see method section).

The c-index, which was computed to quantify the ability of the model to separate events of ACC-specific mortality from patients who did not experience the event, was 0.77 (95\% Cl: $0.73,0.81)$ and 0.77 (95\% Cl: 0.72, 0.81) for the models including and excluding Ki67 in the selection process, respectively. Figure 2 shows the calibration plots of both models. The lines closely follow the ideal line of 45 degrees, indicating that both models are well-calibrated.

Table 2. Initial and internally validated coefficients of the prediction model for ACC-specific mortality.

\begin{tabular}{|c|c|c|c|c|c|c|}
\hline \multirow[t]{2}{*}{ Predictor } & \multicolumn{3}{|c|}{ Model with Ki67 } & \multicolumn{3}{|c|}{ Model without Ki67 } \\
\hline & Coefficient & $\begin{array}{l}\text { HR } \\
(95 \% \mathrm{Cl})\end{array}$ & $\begin{array}{l}\text { Shrunk } \\
\text { Coefficient * }\end{array}$ & Coefficient & $\begin{array}{l}\text { HR } \\
(95 \% \mathrm{Cl})\end{array}$ & $\begin{array}{l}\text { Shrunk } \\
\text { Coefficient * }\end{array}$ \\
\hline $\begin{array}{l}\text { Age at } \\
\text { diagnosis } \\
\text { (year) }\end{array}$ & 0.02 & $\begin{array}{l}1.02 \\
(1.01 \\
1.03)\end{array}$ & 0.02 & 0.02 & $\begin{array}{l}1.02 \\
(1.00 \\
1.03)\end{array}$ & 0.02 \\
\hline $\begin{array}{l}\text { Pathology } \\
\text { positive (yes) }\end{array}$ & 0.52 & $\begin{array}{l}1.68 \\
(0.98 \\
2.89)\end{array}$ & 0.47 & & & \\
\hline $\begin{array}{l}\text { mENSAT } \\
\text { (stage) }\end{array}$ & 0.61 & $\begin{array}{l}1.84 \\
(1.55 \\
2.18)\end{array}$ & 0.65 & 0.66 & $\begin{array}{l}1.93 \\
(1.64 \\
2.28)\end{array}$ & 0.63 \\
\hline Ki67 (\%) & 0.01 & $\begin{array}{l}1, .01 \\
(1.00 \\
1.03)\end{array}$ & 0.01 & & & \\
\hline $\begin{array}{l}\text { Radical } \\
\text { resection of } \\
\text { tumor (yes) }\end{array}$ & -0.37 & $\begin{array}{l}0.69 \\
(0.46 \\
1.04)\end{array}$ & -0.34 & -0.46 & $\begin{array}{l}0.63 \\
(0.42 \\
0.94)\end{array}$ & -0.44 \\
\hline
\end{tabular}



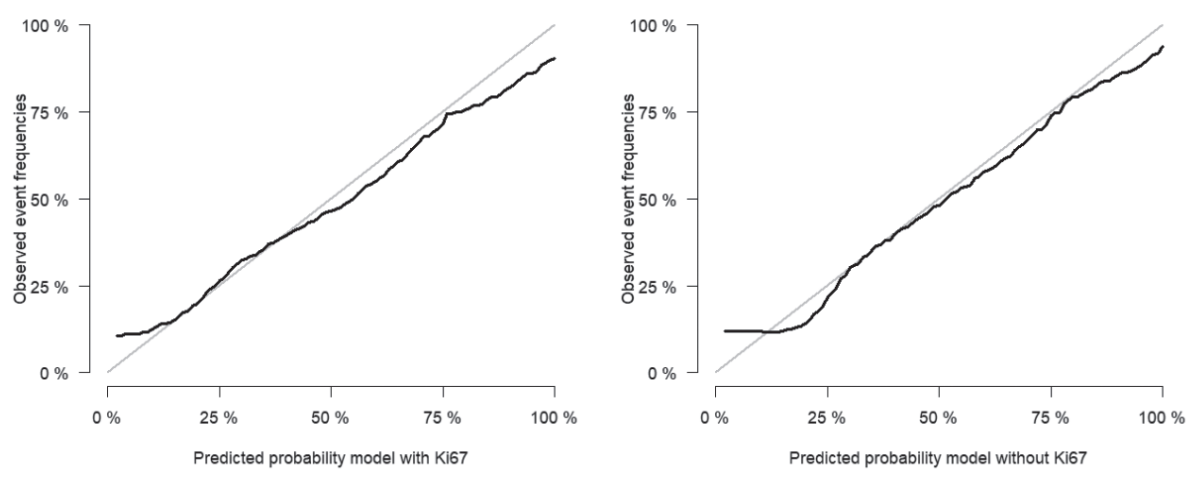

Figure 2. Months of follow-up time.

The internal validation yielded a shrinkage factor of 0.91 for the model with Ki67, and 0.95 for the model without Ki67 (Table 2). Hence, slightly more overfitting was present in the model that included Ki67. In addition, the internal validation yielded a measure of optimism of the c-index of only 0.01 , indicating that the c-index as a measure of the model's ability to discriminate in future patients is estimated to be 0.76 and 0.76 for the two models, respectively (compared to the apparent ability to discriminate of 0.77 and 0.77 , respectively). The shrinkage factors that are close to 1 , together with the small measures of optimism, indicate that these models are not much overfitted. Figures 3 and 4 show the Kaplan-Meier curves for patients stratified by their risk score, for both models. 


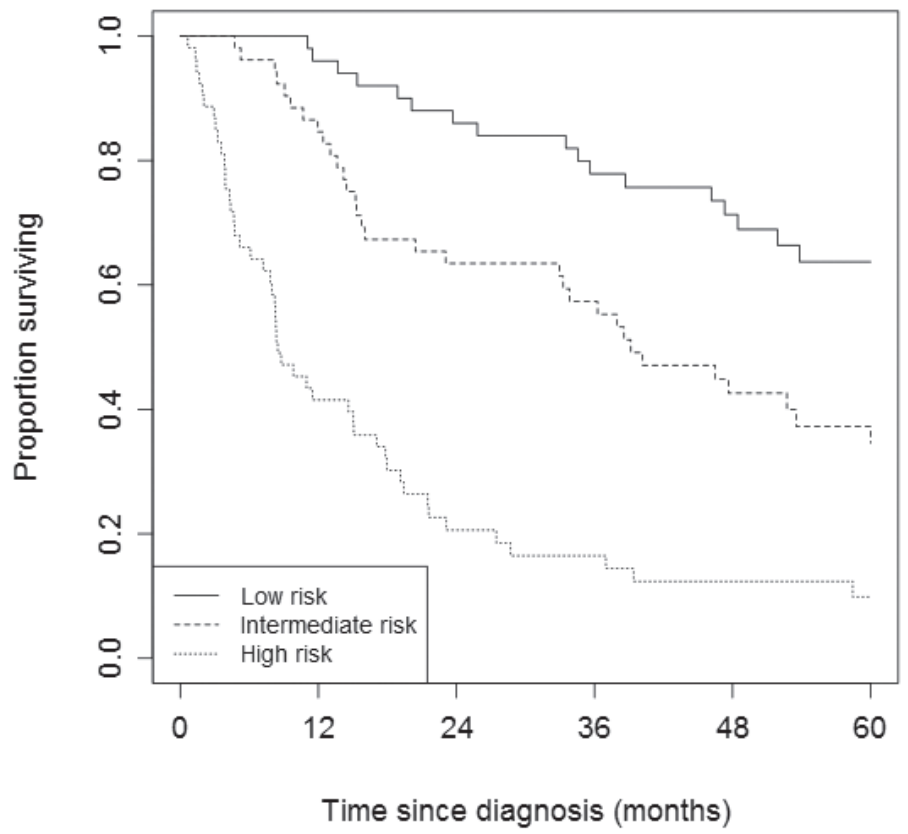

Number at risk

$\begin{array}{rrrrrrl}51 & 48 & 43 & 38 & 31 & 24 \text { (RS }<-0.58 \\ 52 & 44 & 33 & 28 & 19 & 13 \text { (RS }-0.58 \text { to } 0.44 \\ 53 & 22 & 10 & 8 & 6 & 2 \text { (RS }>0.44)\end{array}$

Figure 3. Kaplan-Meier stratified by risk groups based on each individual's risk score (RS) of the model including Ki67. 


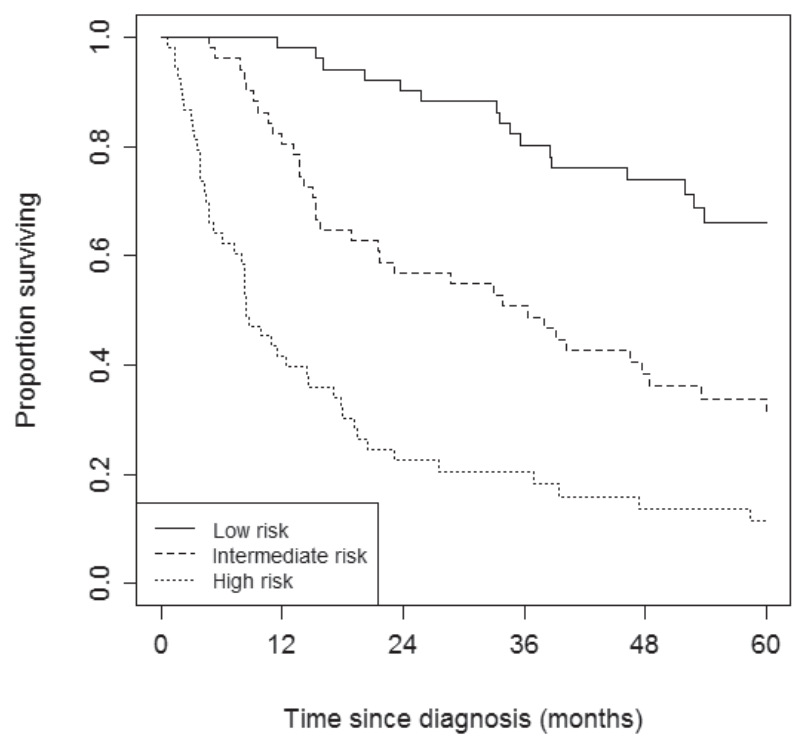

\section{Number at risk}

$\begin{array}{rrrrrc}51 & 50 & 46 & 40 & 32 & 24 \text { (RS }<-0.60 \\ 52 & 41 & 29 & 25 & 18 & 12 \text { (RS }-0.60 \text { to } 0.38 \\ 53 & 22 & 11 & 9 & 6 & 3 \text { (RS }>0.38)\end{array}$

Figure 4. Kaplan-Meier stratified by risk groups based on each individual's risk score (RS) of the model without Ki67. The Risk Score can be computed as 0.02 * (age - 54.5) + 0.63 * (mENSAT - 3.2) - 0.44 * (radical resection of the tumor-0.8).

\section{Prediction for Future Patients}

The prediction models presented in Table 2 can either be used to compute an individual risk score to determine risk category (Figures 3 and 4), or to compute an actual probability that an individual experiences ACC-specific mortality within one, two, or five years. How to do so is outlined below.

The risk score (RS) can be computed by multiplying the shrunk coefficients by the values of an individual patient minus the sample average for age (54.5), mENSAT (3.2), and radical resection (0.8). mENSAT takes on a score from 1-6, with mENSAT I-III scoring 1-3 points, and IVa-IVc scoring 4-6 points. The surgical resection is scored one if radical and zero if not (see method section), for example, using the model without Ki67: 0.02 * (age -54.5$)+0.63$ * (mENSAT - 3.2) $-0.44 *$ (radical resection 0.8 ) of the tumor ( $\mathrm{no}=0$, yes $=1$ ). A 64-year old patient with an mENSAT stage of $4 a$, and no radical resection of the tumor, would have a risk score of 0.02 * $(64-54.5)+$ $0.63 *(4-3.2)-0.44 *(0-0.8)=1.046$. Figure 4 shows that this individual would be classified as being at high risk of ACC-specific mortality. 
The probability of ACC-specific mortality within one, two, or five years is computed by combining the Kaplan-Meier estimate at one, two, or five years and the sum of the shrunk coefficients to the centered values of an individual patient (i.e., the value minus the sample average) as $1-\mathrm{S}(\mathrm{t})^{\exp (\mathrm{LP})}$ : $\mathrm{S}(\mathrm{t})$ is the survival function in which $\mathrm{t}$ is time. S(t) for one, two, and five years is $0.73,0.56$, and 0.37 , respectively (see KaplanMeier Figure 1). $\operatorname{Exp}(\mathrm{LP})$ stands for e raised to the power of the linear predictor (exp: exponential (function), $e$ is the mathematical constant, Ip: lineair predictor). The linear predictor can be computed as 0.02 * (age -54.5$)+0.63$ * (mENSAT - 3.2) - 0.44 * (radical resection of the tumor - 0.8). The 64-year old patient with an mENSAT stage of $4 a$, and no radical resection $(n o=0)$ of the tumor, would have a two-year probability of ACC-specific mortality of $1-0.56^{\exp (\mathrm{LP})}$. LP $=0.02$ * $(64-54.5)+0.63$ * $(4-3.2)-0.44 *(0-0.8)=1.046$; hence, the probability $=1-0.56^{\exp (1.046)}=0.81=$ $81 \%$. This can be confirmed in Figure 4 , in which approximately $20 \%$ of the high-risk group survives past 24 months.

\section{Discussion}

In this collaborative study of the Dutch Adrenal Network, a model capable of predicting ACC-specific mortality was developed. An accurate prediction model could help to identify patients at greater risk of death, and support the decision making on early systemic therapy. Furthermore, a prediction model could support selection of a specific subgroup eligible for new therapeutic compounds.

Ki67 is a suggested prognostic marker in ACC $[5,20]$; therefore, we performed modelling both with and without Ki67. Both models were based on tumor stage defined by the mENSAT classification, age, and radical resection of the primary tumor. The model with Ki67 also relied on hormonal status and the pathology criteria capsular and/or vascular invasion. Although both models showed comparable discriminative ability and calibration, the model with Ki67 was partially based on imputed data because of missing data despite our efforts to revise the Ki67 index for all patients. Previous research has shown that pathology data are often incompletely described in ACC [21]. In addition, pathology reports are not standardized in ACC. Prognostic scoring

systems based on the Weiss and Van Slooten criteria as well as the Ki-67 are to a certain extent subjective, as a reliable assessment largely depends on the expertise of the pathologist [22]. Furthermore, there is often significant inter-observer variability in the determination of the Ki67 [9]. Therefore, in order to improve the generalizability of our prediction model, we decided to use more reliable and easily accessible clinical variables such as age, mENSAT, and completeness of the resection.

This is the first time the mENSAT stage has been considered in a prediction model. Both ENSAT stage, number of affected organs, presence of metastasis, as well as 
nodal status have shown to be correlated with survival $[5,13,23-25]$. The mENSAT stage combines those variables in one staging system, and considers the number of affected organs, including the primary tumor and lymph nodes [5]. The important difference compared with the ENSAT staging system currently used is the fact that T3-4N1M0 is considered stage IV instead of stage III. Lymph node positive disease has been demonstrated to be associated with a less favorable prognosis [26,27], and consequently, given the high recurrence rates for ENSAT stage III, a more prominent role for neo- and adjuvant therapy has been put forward [27]. We endorse using the mENSAT stage in clinical practice.

Estimating survival with the Kaplan-Meier analysis, which is commonly done, has its limitations. It is a nonparametric approach to survival outcomes, and it is able to show univariate relationships graphically or to compute survival factions at a certain time of follow-up. However, the Kaplan-Meier method and the log-rank test cannot be used for multivariate analysis. When looking at current data based on Kaplan Meier data, a stage IV patient could have zero percent change of 5-year survival or almost 20\%. Most patients want to know if they have this small chance at 5-year survival or no chance at all when deciding on starting chemotherapy or mitotane. A clinical prediction model provides such tailored estimation on prognosis. In daily practice, a physician would like to estimate the prognosis tailored for a particular patient, underscoring the need for a reliable clinical prediction model.

In contrast to previously proposed prediction models for ACC, we included pathological data, imputed missing data to prevent a loss of statistical precision, and considered the most recently proposed mENSAT staging system [5]. Another strength of the present study is that our modelling was not based on a pre-selected group of patients with ACC, as our sample included all ENSAT stages. Kim et al. [24] developed a prediction model based on a multi-institutional group of patients treated in the United States who underwent surgery for ACC (Table 3). Notably, they excluded patients with metastatic disease at presentation as well as patients with a macroscopically nonradical resection (R2). Although they studied a relatively large cohort ( $n=148$ ), the external validity of their study is limited because up to $53 \%$ of patients with ACC may present with metastatic disease [21]. Even if their model was solely meant to predict RFS and OS after surgery, they still excluded patients. Surgery is being considered in patients with metastatic disease, and it has been shown that surgery may even improve the outcome in selected patients with stage IV disease, especially if an R0 resection can be achieved [28]. 


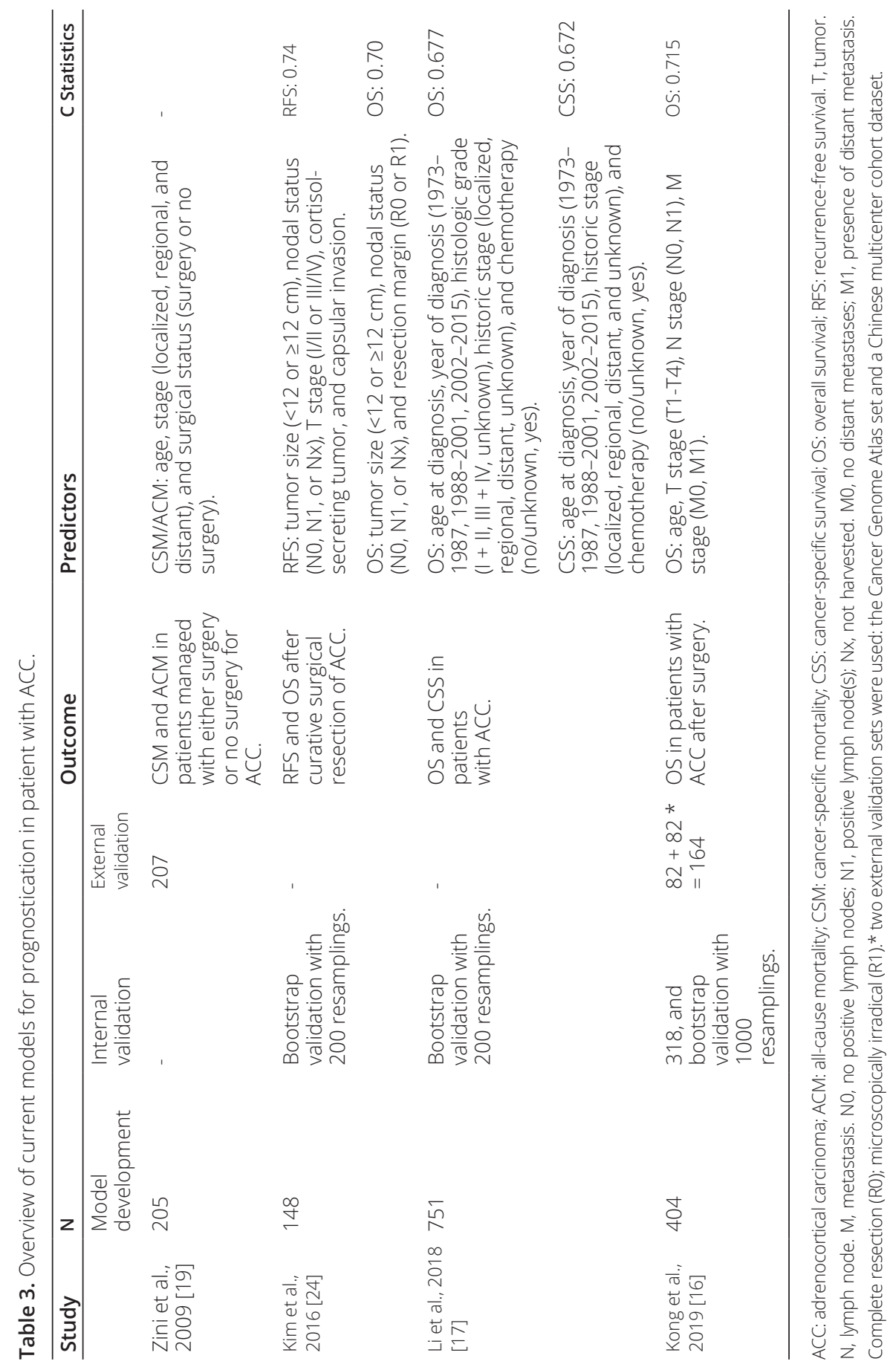


Li et al. presented a nomogram for overall survival (age, year of diagnosis); histologic grade $(I+I I, I I+I V$, and unknown); historic stage (localized, regional, distant, and unknown); chemotherapy (no/unknown or yes); and cancer-specific survival (CSS), including age, year of diagnosis, historic stage and chemotherapy [17]. Kong et al. did not include any histological criteria, but their nomogram included age and TNM stage (according to the 7th American Joint Committee on Cancer (AJCC) TNM staging) [16]. With the nomogram of Li et al., future use causes a problem where year of diagnosis can only be scored till 2015. In addition, their other predictors in that nomogram are not commonly used in clinical practice [17]. The same limitation applies to the nomogram by Kong et al., who use the AJCC TNM staging [16]. Age, however, is a predictor in both models.

It is interesting to note that although the work of Zini et al. [19] suffered from lack of detailed prognostic information, their prediction model included age, stage, and surgery status. In our analysis, we actually include a broad selection of potential predictor variables, and confirm age, stage, and completeness of tumor resection to be significant predictors for ACC specific mortality. So, even in the absence of detailed information, e.g., patients of whom no immunohistochemistry is available, it is possible to make an estimate of cancer-specific mortality.

The study by Kebebew et al. [29] was not designed to develop a prediction model, but their highly powered ( $n=725$ ) multivariable analysis for ACC mortality also showed that ACC stage, surgical resection, and tumor grade (localized, regional, or distant) were independent prognostic factors.

There is a lack of consensus regarding the cut-off point and combination of pathologic criteria that are associated with prognosis [20,30,31]. Consequently, there are limitations to the clinical use of pathology criteria for ACC prognosis. Perhaps in the near future, results of genome and transcriptome studies could be useful. The results of their potential prognostic value seem promising [10,32-34]. For example, hypermethylation of CpG islands, or as a pattern called CpG island methylator phenotype (CIMP), is associated with a poor prognosis in ACC $[10,33,35]$. At the moment, a clearly defined picture of CIMP in ACC is lacking, and although study results on CIMP patterns demonstrate a certain degree of overlap, there are some (methodological) inconsistencies. Furthermore, it is uncertain whether methylation status has the potential to become a prognostic factor on its own, or if it will be an additive to a clinical prediction model, as presented in this study. The latter might be expected considering the fact that DNA methylation is a dynamic process with potential fluctuations over time, and with differences between primary tumor and metastasis. Libbert et al. recently presented a COMBI score, integrating clinical predictors with number of somatic mutations, alterations in the Wnt/beta-catenin and p53/Rb pathways, and promoter region methylation pattern. Again, heterogeneity of 
molecular analysis and definition of cut-off values used pose a problem, but it could be useful as a prognostic determinant in the future.

Individual biomarkers like GOS2 [34] and BUB1, PINK1 [36,37] might overcome the problem of heterogeneity with CIMP, but those biomarkers merely identify ACC with a poor prognosis.

Some limitations of our study merit further mentioning. The prediction model is only applicable to ACC patients of 18 years or older. The cohort we used had a relatively small sample size, which limited the number of predictors for consideration in our model. In view of the fact that the Netherlands currently holds 17 million inhabitants, the size of our study cohort is in agreement with the reported incidence of ACC. In our opinion, the prediction model presented here has the potential to provide a more individualized and practical estimate of the prognosis of ACC, compared to the currently used staging system. Although our model was internally validated using bootstrap validation, external validation is warranted before widespread implementation of the algorithm in a user-friendly prediction calculator.

\section{Materials and Methods}

Data for this retrospective cohort study were obtained from the nine centers of the Dutch Adrenal Network (DAN), as has been described earlier [23]. Patients of $\geq 18$ years who presented with ACC between 1 January 2004 and 31 October 2013 were included. Follow-up data was investigated until 30 June 2016. Because of missing Ki67 data in the original database, an attempt was made to revise the Ki67 index, from 31 January 2018 until 2 April 2020.

\section{Potential Predictor Variables}

We identified potential predictor variables based on clinical reasoning, and on previously published risk factors for mortality. These variables were age at the time of diagnosis [38], sex, body mass index (BMI), TNM classification, hypercortisolism, complaints directly related to tumor mass, venous invasion, ENSAT stage, modified ENSAT (mENSAT) stage [5], radical resection of the tumor (R0 variable "yes"; Rx/R1/R2 variable "no") [20,26], and Ki67 index.

ENSAT stage was defined according to Fassnacht et al. [13]: stage I, tumor size $\leq 5$ $\mathrm{cm}$ (T1NOM0); stage II, tumor size $>5 \mathrm{~cm}$ (T2NOM0); stage III, tumor of any size with at least one of the following factors: tumor infiltration in surrounding tissue (T3), tumor invasion into adjacent organs, or venous tumor thrombus in the vena cava or renal vein (T4), positive lymph node (N1), but no distant metastasis (M0); and stage IV, the presence of distant metastases irrespective of tumor size or lymph node status (T1- 
T4N0-N1M1). The mENSAT classification defines T3-4NOMO as stage III (invasion of surrounding tissues/organs, or invasion of the renal vein or inferior vena cava), and both T3-4N1M0-1 and T3-4NOM1 as stage IV. Stage IV is then subcategorized into Stage IVa (two involved organs), IVb (three involved organs), and IVc (>three involved organs). The primary tumor and " $\mathrm{N}$ " are included as "organ" in the count of number of involved organs [5].

Pathology was scored positive if venous invasion or capsular invasion were present according to the Weiss criteria in the pathology report or capsular and/or vascular invasion was scored yes according to the Van Slooten Index in the pathology report.

Hypercortisolism was defined clinically if this was reported by the treating physician in the patients' file or biochemical with a cortisol level above the upper limit of normal as defined by the hospital laboratory where the patients' cortisol level was analyzed. Cortisol level was determined either in serum, saliva, 24-h urine, and/or during a dexamethasone suppression test.

Complaints due to tumor mass were scored yes if the patient had abdominal pain or back pain that could be due/directly related to the tumor mass.

\section{Immunohistochemistry Revision Ki67}

An attempt was made to revise the Ki67 index for all 160 patients. We were able to track down 55 tumor samples.

To assess the number of cells in cycle (preparing for cell division), selected blocks were stained at one location using the MIB-1 antibody (obtained from Dako, Glostrup, Denmark). All sections were stained in an immunostainer (Ventana Benchmark Ultra, from Roche, Tucson, Arizona, US) in 3 runs. Sections were developed using the Optiview 3,3-diaminobenzidine (DAB) system (also from Roche) as a second step and DAB as chromogen. To assess the number of stained cells, the area with the highest staining intensity was searched for, and that area was magnified using a 40x objective (hot spot method). All cells and all positive cells were counted, and the percentage of positive cells was calculated. A cell was deemed positive when the nucleus was no longer blue. Additionally, faint staining was included as positivity. As a control for the staining effectivity, a tissue microarray was mounted on each individual stained slide.

Unfortunately, information on the Ki67 analytic process used for the other tumor samples in the database is not available because of historical data.

\section{Model Development}

We chose to impute our data to prevent a loss of statistical precision and to decrease 
the likelihood of obtaining biased results [39]. Imputation was performed using stochastic regression imputation with fully conditional specification. Predictive mean matching was used to draw the values to be imputed.

Baseline characteristics of the participants were described using means and standard deviations or absolute numbers and percentages. The overall survival of our cohort was estimated using the Kaplan-Meier method. Median follow-up time was computed, including the first and third quartiles. The median survival time was estimated including 95\% confidence interval (CI) around the median.

We computed a correlation matrix to assess correlations between predictor variables. If variables were highly correlated (Pearson's correlation coefficient, or rho, >0.8), we chose to include only the variable that was deemed more convenient to implement in a clinical prediction tool.

Multivariable Cox proportional hazards regression was used to estimate the coefficients in the prediction model. Backward stepwise elimination was performed to derive a more parsimonious model. We used the Akaike Information Criterion as the rule for deleting variables from the model, which corresponds to a more liberal alpha of 0.157 [40]. The correlation between the scaled Schoenfeld residuals and time was computed for each variable to test the proportional hazards assumption [41].

The performance of the initial prediction model was quantified by measures of discriminative ability and calibration. Discriminative ability is expressed as the c-index, which can take on any value between 0.5 (no discriminative ability) and 1 (perfect discriminative ability), and is an estimate of the probability that of any two randomly chosen patients, the one with the higher prognostic score will outlive the one with the lower prognostic score [41]. Calibration was assessed by visual inspection of the calibration plot. The calibration plot shows the agreement between predicted probabilities and pseudo-observed event status at a follow-up time of two years.

\section{Internal Validation of the Model}

As a rule of thumb, it is suggested that for each potential predictor variable in the model, 10 events should be observed to prevent overfitting. An overfitted model would perform well in the development data, but poorly when applied to new patients. Often, such an overfitted model would produce too extreme predictions. Since our dataset was relatively small, an internal validation step was performed to counteract the possible overfitting of our model to the data. We used standard bootstrapping techniques $(B=1000)$ to obtain optimism-corrected measures of performance (the c-index) and a shrinkage factor. The shrinkage factor is calculated as the optimism-corrected calibration slope. It is a constant between 0 and 1 , and 
the regression coefficients are then shrunk by multiplying by this number. These penalized regression coefficients produce fewer extreme predictions, and hence counteract the effect of overfitting.

Finally, we stratified all patients into three groups based on their predicted risk score (i.e., low, medium, and high risk) for both the model with and the model without the Ki67 index.

\section{Conclusions}

In conclusion, we have developed and internally validated an easy-to-use prediction model for ACC-specific mortality; this model is essentially based on age, mENSAT stage, and completeness of tumor resection. 


\section{References}

1. Kerkhofs, T.M.; Verhoeven, R.H.; Van der Zwan, J.M.; Dieleman, J.; Kerstens, M.N.; Links, T.P.; Van de Poll-Franse, L.V.; Haak, H.R. Adrenocortical Carcinoma: A Population-Based Study on Incidence and Survival in the Netherlands since 1993. Eur. J. Cancer 2013, 49, 2579-2586.

2. Hermsen, I.G.; Gelderblom, H.; Kievit, J.; Romijn, J.A.; Haak, H.R. Extremely Long Survival in Six Patients Despite Recurrent and Metastatic Adrenal Carcinoma. Eur. J. Endocrinol. 2008, 158, 911-919.

3. Lau, S.K.; Weiss, L.M. The Weiss System for Evaluating Adrenocortical Neoplasms: 25 Years Later. Hum. Pathol. 2009, 40, 757-768.

4. Weiss, L.M. Comparative Histologic Study of 43 Metastasizing and Nonmetastasizing Adrenocortical Tumors. Am. J. Surg. Pathol. 1984, 8, 163-169.

5. Libe, R.; Borget, I.; Ronchi, C.L.; Zaggia, B.; Kroiss, M.; Kerkhofs, T.; Bertherat, J.; Volante, M.; Quinkler, M.; Chabre, O.; et al. Prognostic Factors in Stage III-IV Adrenocortical Carcinomas (ACC): An European Network for the Study of Adrenal Tumor (ENSAT) Study. Ann. Oncol. 2015, 26, 2119-2125.

6. van Slooten, H.; Schaberg, A.; Smeenk, D.; Moolenaar, A.J. Morphologic Characteristics of Benign and Malignant Adrenocortical Tumors. Cancer 1985, 55, 766-773.

7. Wachenfeld, C.; Beuschlein, F.; Zwermann, O.; Mora, P.; Fassnacht, M.; Allolio, B.; Reincke, M. Discerning Malignancy in Adrenocortical Tumors: Are Molecular Markers Useful? Eur. J. Endocrinol. 2001, 145, 335-341.

8. Berruti, A.; Fassnacht, M.; Baudin, E.; Hammer, G.; Haak, H.; Leboulleux, S.; Skogseid, B.; Allolio, B.; Terzolo, M. Adjuvant Therapy in Patients with Adrenocortical Carcinoma: A Position of an International Panel. J. Clin. Oncol. 2010, 28, e401-402 (author's reply e403).

9. Papathomas, T.G.; Pucci, E.; Giordano, T.j.; Lu, H.; Duregon, E.; Volante, M.; Papotti, M.; Lloyd, R.V.; Tischler, A.S.; van Nederveen, F.H.; et al. An International Ki67 Reproducibility Study in Adrenal Cortical Carcinoma. Am. J. Surg. Pathol. 2016, 40, 569-576.

10. Assie, G.; Letouze, E.; Fassnacht, M.; Jouinot, A.; Luscap, W.; Barreau, O.; Omeiri, H.; Rodriguez, S.; Perlemoine, K.; Rene-Corail, F.; et al. Integrated Genomic Characterization of Adrenocortical Carcinoma. Nat. Genet. 2014, 46, 607-612.

11. Ettaieb, M.; Kerkhofs, T.; van Engeland, M.; Haak, H. Past, Present and Future of Epigenetics in Adrenocortical Carcinoma. Cancers 2020, 12, 1218.

12. DeLellis, R.A. Pathology and Genetics of Tumours of Endocrine Organs; IARC: Lyon, France, 2004.

13. Fassnacht, M.; Johanssen, S.; Quinkler, M.; Bucsky, P.; Willenberg, H.S.; Beuschlein, F.; Terzolo, M.; Mueller, H.H.; Hahner, S.; Allolio, B. Limited Prognostic Value of the 2004 International Union Against Cancer Staging Classification for Adrenocortical Carcinoma: Proposal for a Revised TNM Classification. Cancer 2009, 115, 243-250.

14. Libé, R. Adrenocortical Carcinoma (ACC): Diagnosis, Prognosis, and Treatment. Front. Cell Dev. Biol. 2015, 3, 45.

15. Koch, A.; Joosten, S.C.; Feng, Z.; de Ruijter, T.C.; Draht, M.X.; Melotte, V.; Smits, K.M.; Veeck, J.; Herman, J.G.; Van Neste, L. Analysis of DNA Methylation in Cancer: Location Revisited. Nat. Rev. Clin. Oncol. 2018, 15, 459-466.

16. Kong, J.; Zheng, J.; Cai, J.; Wu, S.; Diao, X.; Xie, W.; Chen, X.; Liao, C.; Yu, H.; Fan, X. A Nomogram for Individualized Estimation of Survival among Adult Patients with Adrenocortical Carcinoma After Surgery: A Retrospective Analysis and Multicenter Validation Study. Cancer Commun. 2019, 39, 80.

17. Li, Y.; Bian, X.; Ouyang, J.; Wei, S.; He, M.; Luo, Z. Nomograms to Predict overall Survival and Cancer-Specific Survival in Patients with Adrenocortical Carcinoma. Cancer. Manag. Res. 2018, 10, 6949-6959. 
18. Freire, D.S.; Siqueira, S.A.C.; Zerbini, M.C.N.; Wajchenberg, B.L.; Corrêa-Giannella, M.L.; Lucon, A.M.; Pereira, M.A.A. Development and Internal Validation of an Adrenal Cortical Carcinoma Prognostic Score for Predicting the Risk of Metastasis and Local Recurrence. Clin. Endocrinol. 2013, 79, 468-475.

19. Zini, L.; Capitanio, U.; Jeldres, C.; Lughezzani, G.; Sun, M.; Shariat, S.F.; Isbarn, H.; Arjane, P.; Widmer, H.; Perrotte, P. External Validation of a Nomogram Predicting Mortality in Patients with Adrenocortical Carcinoma. BJU Int. 2009, 104, 1661-1667.

20. Beuschlein, F.; Weigel, J.; Saeger, W.; Kroiss, M.; Wild, V.; Daffara, F.; Libe, R.; Ardito, A.; Al Ghuzlan, A.; Quinkler, M.; et al. Major Prognostic Role of Ki67 in Localized Adrenocortical Carcinoma After Complete Resection. J. Clin. Endocrinol. Metab. 2015, 100, 841-849.

21. Assie, G.; Antoni, G.; Tissier, F.; Caillou, B.; Abiven, G.; Gicquel, C.; Leboulleux, S.; Travagli, J.P.; Dromain, C.; Bertagna, X.; et al. Prognostic Parameters of Metastatic Adrenocortical Carcinoma. J. Clin. Endocrinol. Metab. 2007, 92, 148-154.

22. Volante, M.; Buttigliero, C.; Greco, E.; Berruti, A.; Papotti, M. Pathological and Molecular Features of Adrenocortical Carcinoma: An Update. J. Clin. Pathol. 2008, 61, 787-793.

23. Ettaieb, M.H.; Duker, J.C.; Feelders, R.A.; Corssmit, E.P.; Menke-van der Houven van Oordt, C.W.; Timmers, H.J.; Kerstens, M.N.; Wilmink, J.W.; Zelissen, P.M.; Havekes, B.; et al. Synchronous vs. Metachronous Metastases in Adrenocortical Carcinoma: An Analysis of the Dutch Adrenal Network. Horm. Cancer. 2016, 7, 336-344.

24. Kim, Y.; Margonis, G.A.; Prescott, J.D.; Tran, T.B.; Postlewait, L.M.; Maithel, S.K.; Wang, T.S.; Evans, D.B.; Hatzaras, I.; Shenoy, R. Nomograms to Predict Recurrence-Free and overall Survival After Curative Resection of Adrenocortical Carcinoma. JAMA Surg. 2016, 151, 365-373.

25. Lughezzani, G.; Sun, M.; Perrotte, P.; Jeldres, C.; Alasker, A.; Isbarn, H.; Budaus, L.; Shariat, S.F.; Guazzoni, G.; Montorsi, F.; et al. The European Network for the Study of Adrenal Tumors Staging System is Prognostically Superior to the International Union Against Cancer-Staging System: A North American Validation. Eur. J. Cancer 2010, 46, 713-719.

26. Bilimoria, K.Y.; Shen, W.T.; Elaraj, D.; Bentrem, D.J.; Winchester, D.J.; Kebebew, E.; Sturgeon, C. Adrenocortical Carcinoma in the United States. Cancer 2008, 113, 3130-3136.

27. Do, C.C.; Leboulleux, S.; Borget, I.; Bertherat, J.; De la Fouchardiere, C.; Hecart, A.; Caron, P.; Tabarin, A.; Niccoli, P.; Rohmer, V. First Prognostic Analysis of Stage III Adrenocartical Carcinoma Patients After Complete Resection: A Retrospective French Multicentric Study from the INCACOMETE Network. In Proceedings of the 13th European Congress of Endocrinology, Rotterdam, The Netherlands, 30 April-4 May 2011.

28. Dy, B.M.; Strajina, V.; Cayo, A.K.; Richards, M.L.; Farley, D.R.; Grant, C.S.; Harmsen, W.S.; Evans, D.B.; Grubbs, E.G.; Bible, K.C.; et al. Surgical Resection of Synchronously Metastatic Adrenocortical Cancer. Ann. Surg. Oncol. 2015, 22, 146-151.

29. Kebebew, E.; Reiff, E.; Duh, Q.Y.; Clark, O.H.; McMillan, A. Extent of Disease at Presentation and Outcome for Adrenocortical Carcinoma: Have we made Progress? World J. Surg. 2006, 30, 872 878.

30. Stojadinovic, A.; Brennan, M.F.; Hoos, A.; Omeroglu, A.; Leung, D.H.; Dudas, M.E.; Nissan, A.; Cordon-Cardo, C.; Ghossein, R.A. Adrenocortical Adenoma and Carcinoma: Histopathological and Molecular Comparative Analysis. Mod. Pathol. 2003, 16, 742-751.

31. Morimoto, R.; Satoh, F.; Murakami, O.; Suzuki, T.; Abe, T.; Tanemoto, M.; Abe, M.; Uruno, A.; Ishidoya, S.; Arai, Y.; et al. Immunohistochemistry of a Proliferation Marker Ki67/MIB1 in Adrenocortical Carcinomas: Ki67/MIB1 Labeling Index is a Predictor for Recurrence of Adrenocortical Carcinomas. Endocr. J. 2008, 55, 49-55. 
32. Jouinot, A.; Assié, G.; Libé, R.; Fassnacht, M.; Papathomas, T.; Barreau, O.; De La Villeon, B.; Faillot, S.; Hamzaoui, N.; Neou, M.; et al. DNA methylation is an independent prognostic marker of survival in adrenocortical cancer. J. Clin. Endocrinol. Metab. 2016, 102, 923-932, doi:10.1210/jc.2016-3205.

33. Zheng, S.; Cherniack, A.D.; Dewal, N.; Moffitt, R.A.; Danilova, L.; Murray, B.A.; Lerario, A.M.; Else, T.; Knijnenburg, T.A.; Ciriello, G. Comprehensive Pan-Genomic Characterization of Adrenocortical Carcinoma. Cancer Cell 2016, 29, 723-736.

34. Mohan, D.R.; Lerario, A.M.; Else, T.; Mukherjee, B.; Almeida, M.Q.; Vinco, M.; Rege, J.; Mariani, B.M.; Zerbini, M.C.N.; Mendonca, B.B. Targeted Assessment of G0S2 Methylation Identifies a Rapidly Recurrent, Routinely Fatal Molecular Subtype of Adrenocortical Carcinoma. Clin. Cancer Res. 2019, 25, 3276-3288.

35. Barreau, O.; Assie, G.; Wilmot-Roussel, H.; Ragazzon, B.; Baudry, C.; Perlemoine, K.; Rene-Corail, F.; Bertagna, X.; Dousset, B.; Hamzaoui, N.; et al. Identification of a CpG Island Methylator Phenotype in Adrenocortical Carcinomas. J. Clin. Endocrinol. Metab. 2013, 98, E174-E184.

36. De Reynies, A.; Assie, G.; Rickman, D.S.; Tissier, F.; Groussin, L.; Rene-Corail, F.; Dousset, B.; Bertagna, X.; Clauser, E.; Bertherat, J. Gene Expression Profiling Reveals a New Classification of Adrenocortical Tumors and Identifies Molecular Predictors of Malignancy and Survival. J. Clin. Oncol. 2009, 27, 1108-1115.

37. Fragoso, M.C.B.; Almeida, M.Q.; Mazzuco, T.L.; Mariani, B.M.; Brito, L.P.; Gonçalves, T.C.; Alencar, G.A.; Lima, L.d.O.; Faria, A.M.; Bourdeau, I. Combined Expression of BUB1B, DLGAP5, and PINK1 as Predictors of Poor Outcome in Adrenocortical Tumors: Validation in a Brazilian Cohort of Adult and Pediatric Patients. Eur. J. Endocrinol. 2012, 166, 61.

38. Ayala-Ramirez, M.; Jasim, S.; Feng, L.; Ejaz, S.; Deniz, F.; Busaidy, N.; Waguespack, S.G.; Naing, A.; Sircar, K.; Wood, C.G.; et al. Adrenocortical Carcinoma: Clinical Outcomes and Prognosis of 330 Patients at a Tertiary Care Center. Eur. J. Endocrinol. 2013, 169, 891-899.

39. van Kuijk, S.M.; Viechtbauer, W.; Peeters, L.L.; Smits, L. Bias in Regression Coefficient Estimates when Assumptions for Handling Missing Data are Violated: A Simulation Study. Epidemiol. Biostat. Public Health 2016, 13.

40. Steyerberg, E. Clinical Prediction Models: A Practical Approach to Development, Validation, and Updating; Springer Science \& Business Media: Berlin, Germany, 2008.

41. Harrell, F. Regression Modeling Strategies: With Applications to Linear Models, Logistic and Ordinal Regression, and Survival Analysis; Springer: Berlin, Germany, 2015. 
Part II

Treatment strategies 


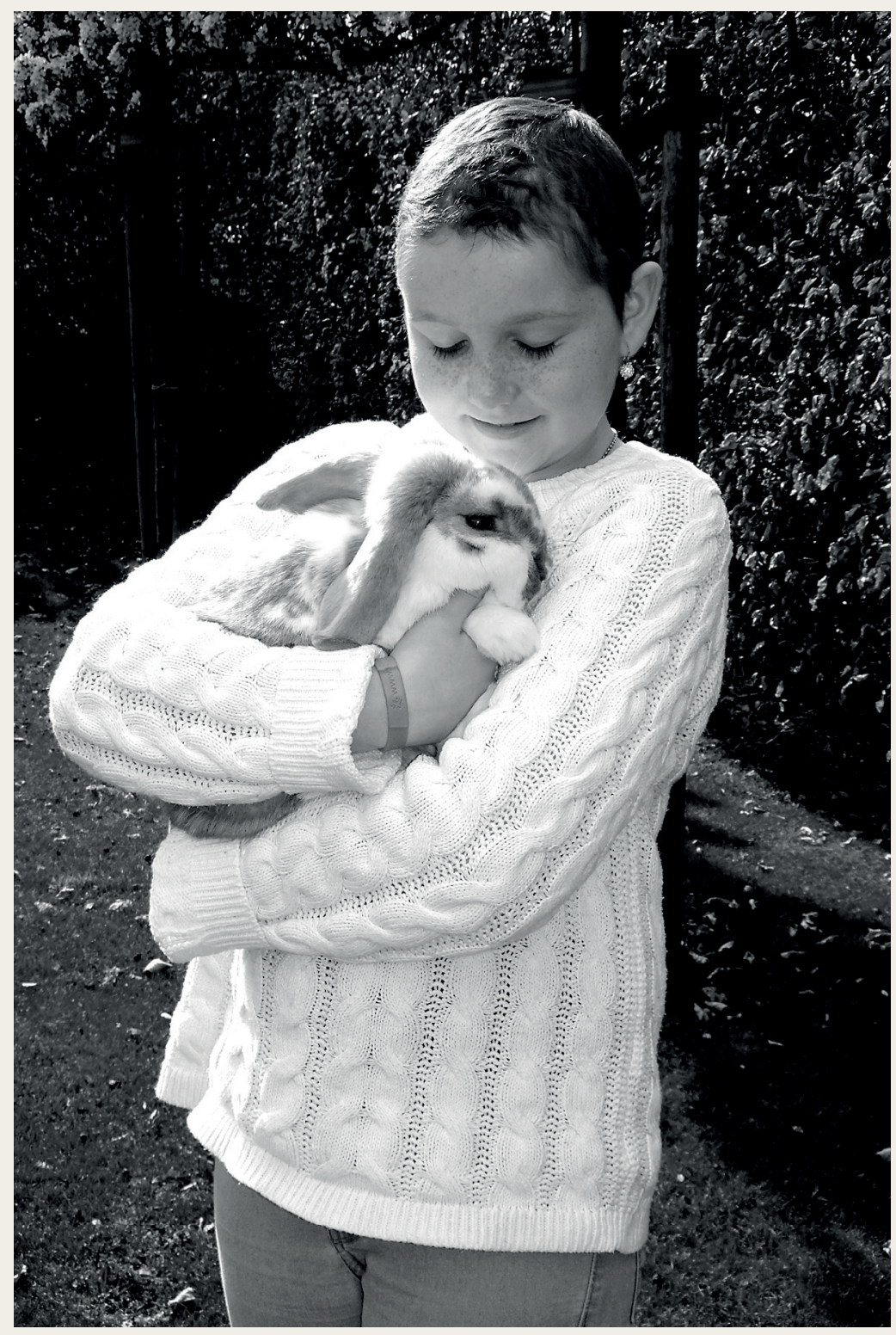




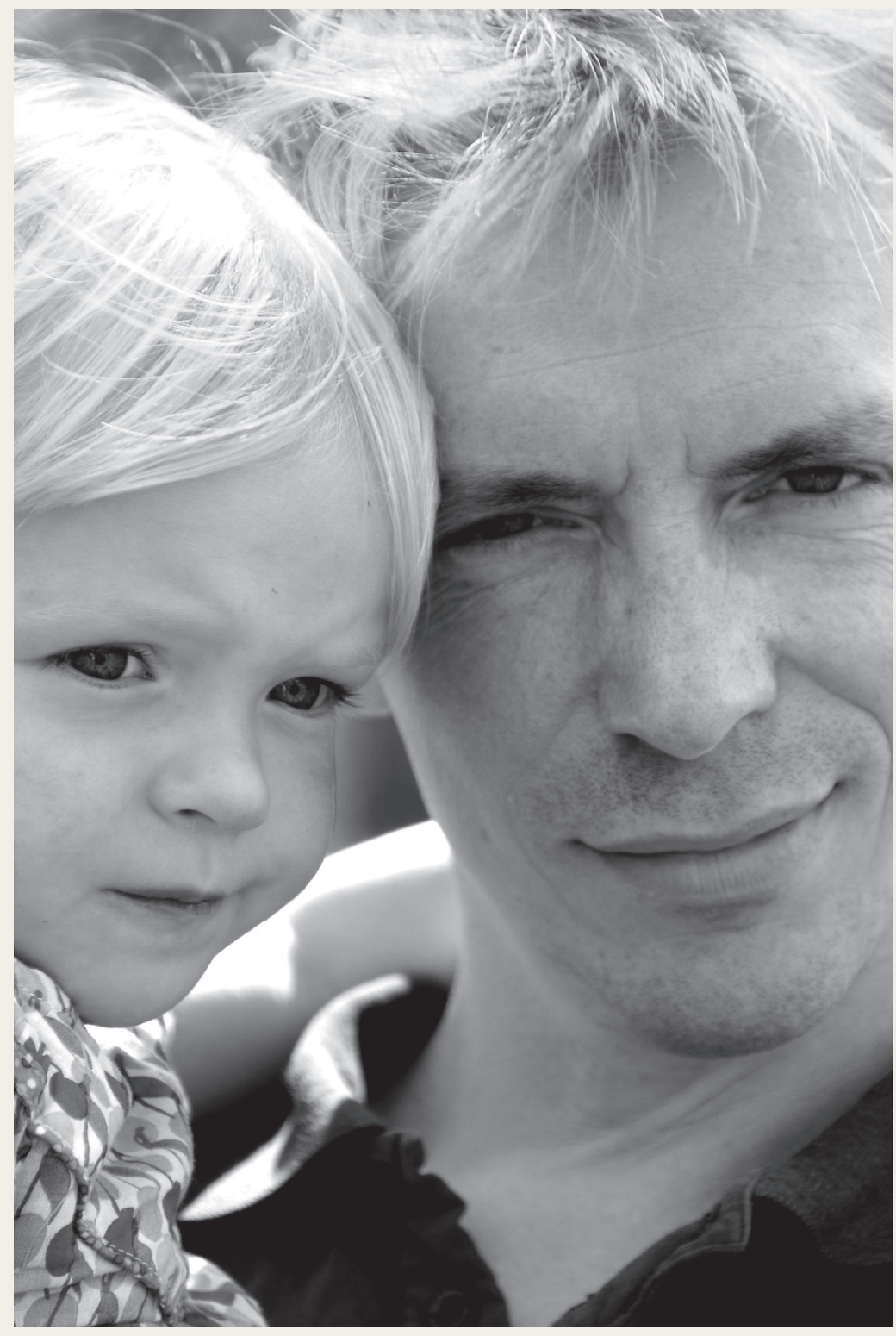




\section{Chapter 04}

Population pharmacokinetic and pharmacogenetic analysis of mitotane in patients with adrenocortical carcinoma: towards individualized dosing

Anyue Yin*, Madeleine H.T. Ettaieb*, Jesse J. Swen, Liselotte van Deun, Thomas M.A. Kerkhofs, Robert J.H.M. van der Straaten, Eleonora P.M. Corssmit, Hans Gelderblom, Michiel N. Kerstens, Richard A. Feelders, Marelise Eekhoff, Henri J.L.M. Timmers, Antonio D'Avolio, Jessica Cusato, Henk-Jan Guchelaar1, Harm R. Haak, Dirk Jan A.R. Moes

* These authors contributed equally.

Clinical Pharmacokinetics June 2020 


\section{Background and Objectives}

Mitotane is the only approved treatment of patients with adrenocortical carcinoma (ACC). A better explanation of the variability in pharmacokinetics (PK) of mitotane and optimization and individualization of mitotane treatment are desirable for patients. This study aims to develop a population pharmacokinetic (PopPK) model to characterize and predict the PK profiles of mitotane in patients with ACC, as well as to explore the effect of genetic variation on mitotane clearance. Ultimately, we aim to facilitate mitotane dose optimization and individualization for patients with ACC.

\section{Methods}

Mitotane concentrations data and dosing data were collected retrospectively from the medical records of patients with ACC taking mitotane orally and participating in the Dutch Adrenal Network. PopPK modelling analysis was performed with NONMEM (version 7.4.1). Genotypes of drug enzymes and transporters, patients' demographic information, and clinical characteristics were investigated as covariates. Subsequently, simulations were performed for optimizing treatment regimens.

\section{Results}

A two-compartment model with first-order absorption and elimination best described the PK data of mitotane collected from 48 patients. Lean body weight (LBW), genotypes of CYP2C19*2 (rs4244285), SLCO1B3 699A>G (rs7311358), and SLCO1B1 $571 T>C$ (rs4149057) were identified to affect mitotane clearance (CL/F) significantly, which decreased the coefficient of variation (CV\%) of random individual variability of CL/F from $67.0 \%$ to $43.0 \%$. Fat amount (i.e. body weight - LBW) was identified to affect the central distribution volume significantly. Simulation results indicated that determining the starting dose with the developed model is beneficial to shorten the period to reach the therapeutic target and limit the risk of toxicity. A regimen that can effectively maintain mitotane concentration within $14-20$ mg/L was established.

\section{Conclusions}

A two-compartment PopPK model well characterized mitotane PK profiles in patients with ACC. Enzyme CYP2C19 and transporter SLCO1B1 and SLCO1B3 may play roles in mitotane disposition. The developed model is beneficial to optimize mitotane treatment schedules and to individualize the initial dose for patients with ACC. Further validation of these finding is still required. 


\section{Introduction}

Adrenocortical carcinoma (ACC) is a rare endocrine malignancy (1 per million per year) with a poor prognosis and limited treatment options[1]. Mitotane, a highly lipophilic compound, is the only approved treatment of ACC by the Food and Drug Administration and the European Medicine Agency[1]. Mitotane is developed as an orally administered treatment and its absorption is improved by concomitant intake of fat-rich food[2]. The bioavailability of mitotane is around $35-40 \%[3]$. Mitotane has a high volume of distribution and the primary distribution site is fat[3, 4]. The half-life of mitotane elimination ranges from 18 to 159 days with a median of 53 days[2, 3].

The efficacy and toxicity of mitotane are related to the plasma concentration[1, 3]. In order to ensure efficacy and avoid increased toxicity, mitotane plasma concentration should be between the therapeutic range of $14-20 \mathrm{mg} / \mathrm{L}$, which requires therapeutic drug monitoring (TDM)[1].

However, due to the large distribution volume and long half-life of mitotane, a longtime interval (around 3-5 months[1]) is usually required for patients to reach the effective concentration[3], which limits the clinical utility of mitotane. The inability to reliably predict mitotane plasma concentrations may result in a prolonged time to reach the target value, hence causing a significant delay in tumor treatment, or may give rise to drug toxicity. In addition, it has been demonstrated that only half of the patients who received a high-dose regimen for 3 months achieved the target[5], suggesting a demand for individualized treatment and a presence of high interindividual variability in the pharmacokinetics (PK) of mitotane. Currently, the dosage titration is largely expert-based making it prone to errors. Therefore, a tool enabling mitotane concentration prediction and an optimized treatment regimen for individual patients, which shortens the period required to reach the target concentration while limiting the toxicity, would be desirable for patients with ACC.

Population PK (PopPK) modelling approach with mixed-effect models enables a quantitative characterization and prediction of drug PK profiles for both the study population and individuals [6]. The development of a PopPK model of mitotane would be beneficial for the characterization and understanding of mitotane PK, as well as for the optimization and personalization of mitotane treatment. Up until now, two studies have performed PopPK modelling analysis on mitotane in patients with ACC[3, 7]. One-compartment models were developed in these two studies. One assuming a self-induced clearance and the body mass index (BMI) was identified to be a covariate of mitotane distribution volume[3]. The other identified the effects of triglyceride and high density lipoprotein on mitotane clearance[7]. Another model-based PK study of mitotane developed a three-compartment model and showed weak correlations of age, gender, body weight, height, and body surface area with model parameters[8]. 
In order to further elucidate the variability of mitotane PK, it would be beneficial to explore the effect of pharmacogenetic polymorphisms[8]. Although the exact PK pathway of mitotane and the enzymes involved in mitotane metabolism remain unknown[9], two studies suggested possible roles for CYP2B6 and CYP2C9[10, 11]. One study demonstrated that the genotype of CYP2B6*6 (rs3745274) was significantly correlated with mitotane plasma concentrations at 3 and 6 months after initiation of treatment[10]. The other study showed that one patient with high mitotane concentration was an CYP2C9 intermediate metabolizer[11]. Further analysis of the relationship between genes encoding for pharmacokinetic enzymes and transporters and mitotane PK profiles, and incorporating these variables into a PopPK model may allow better explanation of mitotane PK variability.

In the current study, a PopPK analysis was performed for mitotane in patients with ACC utilizing the retrospectively collected PK data. The effect of genes encoding drug absorption, distribution, metabolism, and elimination (ADME), patients' demographic information, and clinical characteristics on mitotane PK were investigated as covariates. We aimed to develop a PopPK model to describe and predict the PK of mitotane in patients with ACC, as well as to explore the effect of genetic variation on mitotane clearance. Moreover, we intended to better explain mitotane PK variability with the developed model and to facilitate treatment optimization and individualization for patients with ACC.

\section{Methods}

\subsection{Patients}

Forty nine adult patients diagnosed with ACC ( $\geq 18$ years old), who were enrolled in Dutch Adrenal Network registry, had been treated with mitotane, with consent, and with available mitotane dosing information as well as concentration data were included in this PopPK analysis. One patient was eventually excluded because of the missing starting dosing information.

The study was approved by the Medical Ethical Committee of the Máxima Medical Center Veldhoven (2015), and the approval for inclusion of patients in other institutes was obtained from their local boards. All required informed consents were obtained from all patients. All procedures performed in this study were in accordance with the ethical standards of the institutional medical ethical committee and the 1964 Helsinki Declaration.

\subsection{Pharmacokinetics data}

Data on mitotane plasma concentrations, including concentrations from routine TDM, data sampled during one treatment interval, and data collected after treatment 
discontinuation, as well as all mitotane dosing data were collected retrospectively from patients medical records. Patients administrated mitotane orally and were advised to take mitotane with fat-rich food. The concomitant medication information was not included in the current analysis since the data was not complete. The mitotane plasma concentrations were determined by a validated gas-chromatography/mass spectrometry assay at the Department of Clinical Pharmacy and Toxicology of Leiden University Medical Center (LUMC) [12]. The lower limit of quantification (LLOQ) was $2 \mathrm{mg} / \mathrm{L}$. In addition, patients' demographic information, including age, gender, body weight (WT) and height (HT) at the start of treatment were collected. Furthermore, levels of serum aspartate transaminase (ASAT), alanine transaminase (ALAT), gammaglutamyl transferase (gGT), total cholesterol, and estimated glomerular filtration rate (GFR) (recorded as 0 if the result was $60 \mathrm{~mL} / \mathrm{min} / 1.73 \mathrm{~m}^{2}$ and otherwise 1) were also collected in our analysis.

Lean body weight (LBW) and fat amount (FAT) were also calculated for each patient. LBW was estimated with the Boer formula[13] and FAT was obtained by subtracting LBW from WT.

\subsection{Genotyping method}

DNA of included patients was isolated from EDTA blood samples using Maxwell (Promega, Leiden, the Netherlands) or Magnapure compact (Roche, Almere, the Netherlands). Genotyping of patients was performed with Drug Metabolizing Enzymes and Transporters (DMET'M ) plus array (Affymetrix UK Itd, High Wycombe, United Kingdom), which contains 1936 genetic variants (1931 single nucleotide

polymorphisms (SNPs) and 5 copy number variations (CNVs)) of ADME-related enzymes and transporters[14], according to manufacturers' protocol. The method was described in detail previously[15, 16].

A pre-set selection was performed using the DMET ${ }^{\mathrm{TM}}$ console software that generates fully annotated marker reports based on a translation file as recommended by Affymetrics ${ }^{\circledR}[17]$. The reports include commonly recognized, haplotype-based allele calls commonly cited in Medline reference studies[18-20]. The DMET ${ }^{\mathrm{TM}}$ Plus allele translation software produces a comprehensive genotyping report containing pharmacogenomic reference data on all probes. This step leads to a selection of 959 SNPS from the total of 1931 SNPs present on the DMETTM platform. Subsequently, the SNPs that deviated from Hardy-Weinberg equilibrium $(p<0.0001)$, with call rate below 97\%, or with a minor allele frequency (MAF) $<0.1$, as well as tri-allelic SNPS and SNPS of genes located on X chromosome were excluded from further analysis. 


\subsection{Population PK model development}

Based on the obtained mitotane concentration data, a non-linear mixed-effects model was developed. Parameters were estimated by using the first order conditional estimation method with interaction (FOCEI) implemented in NONMEM software, version 7.4.1 (ICON Development Solutions). One-, two- and three-compartment models, with first-order absorption and first-order elimination, were explored as the structural model. Data points below LLOQ were omitted since they only contributed to $3.6 \%$ of the observations [21, 22].

Since the majority of collected data were trough concentrations and data concerning the absorption phase was limited, absorption rate constant (KA) was first estimated based on a sub-dataset containing data of the patients who contributed multiple data points during one treatment interval at steady state. The estimate of KA was then fixed to analyze the full dataset. Inter-occasion variability (IOV) was incorporated on apparent systematic clearance (CL/F) and every 200 days of treatment was defined as an occasion. In addition, to simplify the situation, all patients were assumed to receive a single dose once a day at 8:00 AM with the dose amount being equal to the total daily dose.

Further detailed description of the PopPK modelling methods is shown in Online Resource 1.

\subsection{Identify potential correlated SNPs and covariate analysis}

Since knowledge about the relationship between mitotane clearance and pharmacogenetic polymorphisms is limited, an exploratory analysis was first performed to find potential SNPs which were correlated with mitotane clearance. The estimates of random inter-individual variability (IIV) of CL/F () from the basic model and the genotyping results were utilized. For each SNP, when the number of patients in a minor homozygous group was less than 4, the results of these patients were combined with the corresponding heterozygote group for the association analysis assuming a dominant allele effect. Additionally, when the number of patients with genotype results of Zero Copy Number or Possible Rare Allele was less than 4 or when patients had NoCall results, the results were not included for statistical analysis. One-way ANOVA test and two sided t-test were performed with R (version 3.6.1; R Foundation for Statistical Computing, Vienna, Austria) to evaluate the difference of across genotype groups for each SNP. The selection of test method depended on the number of genotype groups of each SNP after the combination. The SNPs were considered to correlate with mitotane clearance if the p-value is lower than 0.05 . The correction for multiple testing was not performed because of the exploratory characteristic of the current analysis. 
The identified SNPS, as well as patients' demographic information and clinical characteristics were considered in covariate analysis. Stepwise covariate modelling (SCM) function implemented with Perl-Speaks-NONMEM (version 4.7.0) [23] was applied. Both a forward inclusion $(p<0.05)$ and a backward elimination process $(p<0.01)$ were performed to identify significant covariates. More detailed description of the covariates analysis is shown in Online Resource 1.

\subsection{Model evaluation}

The predictability and stability of the final model was evaluated with goodnessof-fit (GOF) plots, prediction-corrected virtual predicted check (pcVPC)[24], and nonparametric bootstrap. Normalized prediction distribution error (NPDE) were also applied for evaluation. All figures were created with R. Detailed description of the evaluation methods is shown in Online Resource 1.

\subsection{Simulations for treatment optimization}

Based on the final model, simulations were performed to optimize mitotane dosing regimen and starting dose determination, in order to shorten the target reaching time while limiting the risk of toxicity. The simulation was performed for patients included in this study, as they are considered to be able to represent the corresponding adult patients population. The individual parameters of each patient were used to simulate the "real" mitotane concentrations $\left(C_{\text {sim_real }}\right)$ under each regimen. The residual errors were not considered. Different strategies of adjusting the dose according to $C_{\text {sim_real }}$ are shown in Fig. 1. All simulations were performed by $\mathrm{R}$ and the differential equations were solved using RxODE package (version 0.6-1)[25]. Detailed description of the regimens and simulation methods are shown in Online Resource 1.

On the basis of the simulated PK curves, for patients who originally reached the target, the mean and max time needed to reach the target (, the first day when $C_{\text {sim_real }}$ $14 \mathrm{mg} / \mathrm{L})$, the mean percentage of days when $C_{\text {sim_real }}$ were higher than $20 \mathrm{mg} / \mathrm{L}$ in the first 200 days (), and the mean percentages of $C_{\text {sim_real }}$ located outside the therapeutic window after reaching the target (), were calculated and compared across different strategies. represents a probability of causing toxicity in the early phase of treatment and represents the ability of maintaining the concentration within therapeutic window. Meanwhile, the median maximum and minimum $C_{\text {sim_real, }}$ as well as the range of determined starting dose were also collected and evaluated. As an optimized regimen is expected to be able to ensure a quicker target reaching and well maintain the concentration within the therapeutic window while not causing much toxicity, the optimization target was defined as the mean 90 days (3 months), the mean 10\%, 
and the mean $15 \%$.

Using the optimized regimen, a Shiny app was created based on the shiny package (version 1.4.0) and RxODE package in $\mathrm{R}$ in order to perform simulation for a random patient and to elucidate an option of providing treatment advice for a new patient based on the model. The detailed description is shown in Online Resource 2.

\section{Results:}

\subsection{Patients and data}

Data from 48 patients with ACC (21 males and 27 females) were included in the PopPK analysis. The characteristics of patients are summarized in Table 1. Patients received mitotane treatment between 2002-2017 and the median duration of treatment was 713.5 days (range from 90-2856 days). The total daily dosage ranged from $0.5 \mathrm{~g}-16 \mathrm{~g}$ per day and was divided into one to four doses. Five (2 patients), six (1 patient), and eight (1 patient) daily dosages were also applied occasionally. Fortyone patients reached the concentration target during treatment, among whom 16 patients reached the target after 150 days. In total, 914 concentration data points were collected from patients' electronic hospital records, 33 of which were below the LLOQ. The time-course of collected mitotane concentrations was shown in Fig. 2. Nine patients contributed multiple sampling data within one treatment interval and 13 patients have more than one data point collected after treatment discontinuation. The median number of data points contributed by each patient was 16.5 , ranging from 2 to 47.

\subsection{The basic model}

Based on the sub-dataset containing data from the 9 patients with multiple sampling data within one treatment interval, the KA was estimated as 22.1 (/day) and 15.0 (/day) under a one-compartment and a two-compartment model structure, respectively. A three-compartment model could not be identified since: 1) the time-course of mitotane concentration did not meet the characteristics of a three-compartment model; 2) when running the three-compartment model, the parameters were shown to be unidentifiable. The basic models were then developed by fitting the full dataset with fixed KA and incorporating IOV on CL/F. Relative standard error (RSE) of parameter estimates of both two model structures were all within the acceptable range $(<30 \%)$. The objective function value (OFV) of the two-compartment model reduced 92.13 compared with that of the one-compartment model $(p<0.001$, degree of freedom $=4)$, suggesting an improvement on the model fitness. Therefore, the two-compartment model was ultimately selected for describing mitotane PK profiles in patients with 
ACC in this study. The model structure is shown in Online Resource 1, Fig. S1. The parameter estimates of the basic model were shown in Table 2. High percentage coefficient of variation (CV\%) of IIV for all parameters were identified and the CV\% of IIV for apparent distribution rate constant (Q/F) was even higher than 100\%.

\subsection{Pharmacogenetic analysis}

For each patient, the genotyping results of the 959 SNPS from the DMET ${ }^{T M}$ platform were obtained. A list of the these SNPs can be found in Online Resource 3. All SNPS were in Hardy-Weinberg equilibrium (p0.0001). The flow diagram of the genetic variants selection is shown in Fig. 3. Eventually, 172 SNPS were included for the further investigation.

Among the 172 SNPs, 55 SNPs had less than 4 patients belonging to the minor homozygous group. The NoCall result was reported in one patient in 19 SNPS and the Possible Rare Allele was reported in one patient in 1 SNP. The results of these patients were thus not included in the association analysis of corresponding SNPS. In contrast, the Zero Copy Number occurred in 3 SNPs in 8, 24, and 24 patients respectively. Thus, patients with Zero Copy Number were treated as a different genotype group in the association analysis of these 3 SNPS.

Finally, the result of the association test showed that 11 SNPS, as is shown in Online Resource 1, Table. S1, were potentially related to mitotane clearance (p0.05). Among these 11 SNPs, the genotyping results of CYP2C18 1154C>T (rs2281891) and CYP2C19*2 (rs4244285) were shown to be 100\% in linkage disequilibrium in our dataset, same as the genotyping results of SLCO1B3 334G>T (rs4149117), 699A>G (rs7311358), and 1557G>A (rs2053098) and that of the 3 SNPs located on VKORC1 $(283+124 G>C, 174-136 C>T$, and $-1639 G>A)$. The results of the identified 11 SNPS were subsequently combined into the full dataset for the stepwise covariate analysis.

\subsection{The final model}

The parameter estimates of the final model are shown in Table 2. Genotypes of CYP2C19*2 (rs4244285), SLCO1B3 699A>G (rs7311358), and SLCO1B1 571T>C (rs4149057), and LBW at the start of treatment with power relation were identified to have significant effect on the CL/F of mitotane (Table 2). Carrying ' $A$ ' variant in CYP2C19*2 reduced the CL/F by 44.9\%, and carrying ' $G$ ' variant in SLCO1B3 699A>G resulted in a 39.9\% reduction of CL/F (Table 2). As for SLCO1B1 571T>C, the CL/F of patients carrying one ' $C$ ' variant decreased to $40.2 \%$ of that of wild type patients, and the CL/F of patients carrying two ' $\mathrm{C}$ ' variants decreased to $30.2 \%$. The distribution of derived from the basic model in each genotype group of above 3 SNPs was shown 
in Online Resource 1, Fig. S2. In addition, FAT at the start of treatment with power relation was identified to influence the apparent distribution volume of central compartment $\left(V_{c} / F\right)$ significantly. The inclusion of these covariates decreased the $\mathrm{CV} \%$ of $C L / F$ and $V_{c} / F$ from $67.0 \%$ and $68.1 \%$ to $43.0 \%$ and $47.2 \%$, respectively. Overall, the parameter estimates showed to be in good agreement with the bootstrap results (Table 2).

The GOF plots (Fig. 4) show that the individual predictions of the final model are in good accordance with the observations, while the population predictions are slightly deviated from the observations. The conditional weighted residual errors (CWRES) randomly distributed around zero without obvious trends over time or across population predictions. The PCVPC plot (Fig. 5) shows that the 5th, 50th, and 95th percentiles of prediction-corrected concentrations can be mostly adequately covered by the $95 \% \mathrm{Cl}$ of the corresponding percentiles of simulations, although a few large prediction-corrected concentrations present. The NPDE results is shown in Online Resource 1, Fig. S3.

\subsection{Simulation results}

The simulation results of different regimens in included patients who originally reached the target $(\mathrm{N}=41)$ are summarized in Table 3.

The previously suggested high-dose regimen (Regimen 1) resulted in the lowest but the highest. The $C_{\text {sim_real }}$ can also not be well maintained within the therapeutic range.

As for the newly designed strategies, if all patients started with the same dosage (Regimen 2-2g, 2-4g and 2-6g), the increase in the starting dosage reduced but increased and weakened the ability of maintaining $C_{\text {sim_real }}$ within the therapeutic range. When determining the starting dose individually (Regimen 3-77day, 3-98day and 3-119day), Regimen 3-98day fulfilled the optimization target and resulted in lower but higher and compared with Regimen 2-4g. The range of determined starting dose was in accordance with what is currently recommended [26] (Table 3).

Compared with Regimen 2-4g and 3-98day, increasing the dose reduction amount to $4 \mathrm{~g}$ when $C_{\text {sim_real }}>20 \mathrm{mg} / \mathrm{L}$ reduced the and, whereas setting $50 \%$ deduction when $C_{\text {sim_real }}>20 \mathrm{mg} / \mathrm{L}$ reduced but increased the (Regimen 4 and 5 ). Both these changes did not affect. In contrast, when adjusting the dose change amount when $C_{\text {sim_real }} 14$ $\mathrm{mg} / \mathrm{L}$, the evaluated regimens did not provide better results (Regimen 6 and 7 ).

Regimen 8, where a constant starting dose determined by the model was applied, provided generally better results compared with starting with $4 \mathrm{~g} / \mathrm{per}$ day for all patients (Regimen 2-4g) in terms of , , and the ability of maintaining concentration within the therapeutic range. The range of suggested starting dose (3 to $7 \mathrm{~g}$, median 
5g) was slightly beyond the current recommended range but was considered to be acceptable. In comparison, when determining a constant dose using individual PK parameters (incorporating IIV estimates) (Regimen 9), the and max decreased. Although increased, it is still low enough. The suggested doses under Regimen 9 were relatively higher (3 to $10 \mathrm{~g}$ ) since IIV was taken into account.

Overall, Regimen 2-4g, 3-98day, 4-(-4g), 4-(-50\%), 5-(-4 g), 7-1, 8, and 9 fulfilled the optimization target. Individualized starting dose resulted in lower but higher compared with fixed starting dose. Regimen 3-98day and 5-(-4g) provided the lowest mean and regimen 5-(-4g) resulted in lower. Regimen 8 provided the lowest and Regimen 9 provided the lowest max and mean . Based on these results, Regimen 5-($4 \mathrm{~g}$ ) and Regimen 8 were considered to be more beneficial, and Regimen 9 could also be applied considering the patients' tolerance to the level of dose increase.

The Shiny app was established based on the final model and treatment strategy 5-($4 \mathrm{~g}$ ) was applied since this regimen provided the lowest mean. An reduced model where the effect of pharmacogenetic variation was not included was also built in to serve as an alternative option for patients when genotyping results are not available. The results are shown in Online Resource 2.

\section{Discussion}

In the current study, a two-compartment PopPK model was developed which adequately described the PK profile of mitotane in patients with ACC. The identified covariates explained $24 \%$ and $20.9 \%$ of random variability in mitotane clearance and distribution volume, respectively. As mitotane distributes in most body tissues and predominantly in the fat[1], the two-compartment model structure is considered to be also in line with the PK characteristics of mitotane, although wide $95 \% \mathrm{Cl}$ of parameter Q/F still indicate an uncertainty in the estimation. A three-compartment model structure, which has been applied previously on mitotane[8], could not be identified in this study as the time-course of mitotane concentration did not meet the characteristics of a three-compartment model and parameter estimates for the three-compartment model were found to be unidentifiable.

Because of the limited data in the absorption phase, KA was first estimated based on a sub-dataset and then fixed to analyze the full dataset. Precise KA estimation was unidentifiable if estimating based on the full dataset. The estimates of $V_{c} / F$ and $V_{p} / F$ are relatively large, which is in accordance with precious reports and the fact that mitotane distributes in many body tissues[1,3]. The separate effects of LBW and FAT on mitotane distribution volumes were of interest in this study as they are more realistic covariates physiologically[3, 4]. As a result, FAT was identified to be a significant covariate on the $V_{c} / F$. The estimated half-life of mitotane in the included patients 
ranged from 16.4 to 700.6 days with a median of 101.5 days. This range is wider than what was reported previously[1,2]. This may be explained by the larger number of patients included in the current study than the original study[2]. Incorporating IOV on CL/F in the current study explained the intra-subject variability. The estimates of IOV indicate an overall increasing clearance during the first 500 days followed by a decrease thereafter (Online Resource 1, Fig. S4). This dynamic indicates that a selfinduction in mitotane clearance, which has been suggested previously[3], may exist temporarily.

The current study for the first time explored and quantified the potential effect of pharmacogenetic variation on mitotane clearance in patients with ACC. Due to the lack of knowledge regarding the PK pathway of mitotane, a wide range of SNPs from DMETTM plus array were considered. However, because of the limited number of patients, it was decided to focus on the SNPS with known functionality by adopting a pre-set selection[17], although an exploratory analysis based on all genetic variants from DMET TM plus array was also performed. The flow diagram of the SNP selection and the 9 additional SNPS that are potentially correlated to mitotane clearance if the pre-set selection was not considered are shown in Online Resource 1, Table S2 and

Fig. S5. Genes located on the X chromosome were excluded since only the general influence of gender on mitotane PK was considered.

Eventually, three SNPS, i.e. CYP2C19*2 (rs4244285), SLCO1B3 699A>G (rs7311358), and SLCO1B1 571T>C (rs4149057), were included in the final model and were considered as the pharmacogenetic polymorphisms that should be considered for mitotane dose selection. This result also suggests that enzyme CYP2C19 and transporters SLCO1B3 and SLCO1B1 for drug uptake in the liver might be involved in mitotane PK pathways, but further confirmation is required.

In fact, CYP2C19*2 was in 100\% linkage disequilibrium with CYP2C18 1154C>T (rs2281891) in our dataset, same as SLCO1B3 699A>G with SLCO1B3 334G>T (rs4149117) and SLCO1B3 1557G>A (rs2053098). Comparable high linkage disequilibrium was also found in 1000 genomes CEU. Compared with CYP2C18 $1154 \mathrm{C}>\mathrm{T}$ for which no sufficient evidence has been found about the effect on the drug PK, the ' $A$ ' variant of CYP2C19*2 is known to be a nonfunctioning variant and has been demonstrated to decrease the activity of CYP2C19[27, 28]. Similarly, the variants of SLCO1B3 699A>G with SLCO1B3 334G>T have been reported to be associated with the decrease of drug clearance and SLCO1B3 699A>G has stronger level of clinical annotations[29, 30]. Therefore, CYP2C19*2 and SLCO1B3 699A>G were included in the final model.

CYP2B6*6, which has been reported to be related to mitotane plasma concentrations detected at 3 and 6 months[10], was not identified to have significant effect on mitotane clearance in the current study. Among the 5 SNPs located on CYP2B6 which 
were included in the association analysis, none of them was significantly related to mitotane clearance $(p>0.05)$. This discrepancy may be due to the much longer observation period in the present study. One SNP located on CYP2C9, CYP2C9*2 (rs1799853), was not identified to be significant either. However, the evidence of the involvement of CYP2C9 is in fact insufficient.

The predictability and stability of the final model were confirmed to be acceptable. In the pcVPC plot, a few prediction-corrected concentrations are inadequately covered by the simulations. A possible explanation is that the observations at corresponding time points are from a single patient and the population prediction of this patient is much smaller than real observations. The deviation of population predictions from observations can also be seen in the GOF plots. Patients' adherence and other unknown factors may also introduce additional bias. Identification of additional covariates, such as the effect of co-medication and food intake, might improve the population predictions.

Based on the final PopPK model, several mitotane treatment strategies were designed and evaluated by simulations. A regimen with bolus dose followed by maintenance dose was not considered as this regimen requires high dosage which is not tolerable for patients. Among the regimens that fulfilled the optimization target, applying individual starting dose determined by the model was demonstrated to shorten the time to achieve the therapeutic window compared with starting with fixed dose for all patients. Under the setting of individualized starting dose, the regimens with stepwise increasing dose at start required less time to reach the therapeutic target, while the one with constant starting dose demonstrated the lowest risk of having toxicity. The determined individual starting dose was also acceptable. In addition, the newly designed dose adjustment strategies were able to satisfactorily keep the mitotane concentrations within the therapeutic range. Therefore, determining the starting dose with the developed model is considered to be most beneficial in terms of shortening the time to reach the therapeutic target and limit the risk of toxicity. However, due to the fact that a shorter is normally paired with a higher, it is suggested to consider based on patients' condition whether the increased risk of having toxicity can be tolerated in order to gain the benefit of a shorter time to reach the therapeutic target when selecting a dosing regimen.

Obtaining individual parameters based on one (or more) TDM result with the PopPK model and determining the dose amount accordingly can also decrease the risk of toxicity while providing a satisfactory target reaching time, thus it is also a promising strategy. However, patients tolerance to the high level of dose increase need to be considered when applying this strategy. This method can also be useful to estimate an adequate dose for the drug concentration level maintenance after reaching the therapeutic window so that to decrease the frequency of dose adaptation. 
Simulation results also indicate that in order to reduce the risk of having toxicity and effectively maintain mitotane concentration within the therapeutic range, a better strategy is to set the concentration boundary of dose decreasing at $18 \mathrm{mg} / \mathrm{L}$ instead of $20 \mathrm{mg} / \mathrm{L}$. This early dose adjustment takes the 7 days' time when the monitoring result is unknown and the dose is not adjusted into consideration. The concentration boundary of dose increasing needs to be $14 \mathrm{mg} / \mathrm{L}$ since it affects the adequacy of maintaining the plasma concentration above $14 \mathrm{mg} / \mathrm{L}$. The frequency of TDM was set at once every 21 days as suggested by the guideline in the simulation. If TDM is performed less frequently, a larger dose change step will be required.

The current study has some limitations. Firstly, the small number of patients included in this study and the exploratory characteristic of this analysis may influence the power of covariate analysis especially for pharmacogenetic analysis. However, as the dataset consisted of concentrations on different occasions for each patient, which enabled differentiation between IIV and intra-subject variability (i.e. IOV) in clearance, the certainty of the possible genotype effect on clearance which is more likely to be covered by IIV since genotype is a constant factor in patients was increased. Nonetheless, further validation with an external dataset to replicate the findings is warranted to confirm the identified associations and to translate the findings into a clinical recommendation. However since the ACC is a very rare disease (1 per million per year), collection of another comparable or even larger dataset will be challenging. Therefore, an in vitro assay might be more feasible in future studies to substantiate the activity of the suggested enzymes in mitotane PK. Secondly, the model lacks a strong ability to accurately predict high concentrations (e.g, peak concentrations) due to the limited data input in the absorption and distribution phase. Furthermore, the accuracy of parameter estimates may be affected by our simplification of multiple daily dosing to a single dose. However, the prediction of mitotane trough concentrations and the suggestion of daily dose based on the model will not be significantly affected. Therefore we believe this model is still fit for the current application. Thirdly, the impact of co-administrated drugs and the food intake on mitotane PK was not was not taken into account in this study due to the lack of data.

In conclusion, the current study presents a two-compartment PopPK model which well characterizes mitotane PK profiles in patients with ACC. The polymorphisms of CYP2C19*2 (rs4244285), SLCO1B3 699G>A (rs7311358), and SLCO1B1 571T>C (rs4149057) are identified to be correlated to mitotane PK. Further external or in vitro evaluation is suggested to confirm the results. Moreover, optimized mitotane treatment schedules for patients with ACC were identified by simulation and the developed model can be of help to individualize the initial dose. These strategies should be confirmed in a prospective study. 
Table 1 Patient characteristics ( $N=48)$

\begin{tabular}{|c|c|c|c|}
\hline Characteristic & Value/Mean & SD & Range \\
\hline \multicolumn{4}{|l|}{ Patient characteristics } \\
\hline Number of patients (N) & 48 & & \\
\hline Gender, Male (N(\%)) & $21(43.8 \%)$ & & \\
\hline Age (years) ${ }^{a}$ & 52.0 & 12.1 & 22.6-76.8 \\
\hline Weight (kg) ( N=2 No record) & 80.0 & 15.9 & $52.5-120$ \\
\hline Height $(\mathrm{cm})^{a}(\mathrm{~N}=5$ No record) & 172 & 10.0 & 154-193 \\
\hline BMI $\left(\mathrm{kg} / \mathrm{m}^{2}\right)(\mathrm{N}=5$ No record $)$ & 27.1 & 4.48 & $18.2-38.3$ \\
\hline LBW $(\mathrm{kg})^{\mathrm{a}}(\mathrm{N}=5$ No record $)$ & 55.8 & 10.0 & 39.7-78.5 \\
\hline ASAT (IU/L) $)^{b}(N=1$ No record) & 45.15 & 35.3 & $16-185$ \\
\hline $\operatorname{ALAT}(I \cup / L)^{b}(\mathrm{~N}=1$ No record $)$ & 42.68 & 35.6 & $9-197$ \\
\hline GammaGT (IU/L) (N=1 No record) & 278.70 & 215.9 & $55-898$ \\
\hline $\begin{array}{l}\text { GFR, }>50 \% \text { normal records }(\mathrm{N}(\%)) \\
\qquad(\mathrm{N}=7 \text { No record })\end{array}$ & 39 (95.1\%) & & \\
\hline Cholesterol $(\mathrm{mmol} / \mathrm{L})^{b}(\mathrm{~N}=11$ No record $)$ & 6.54 & 1.56 & $3.6-11.6$ \\
\hline \multicolumn{4}{|l|}{ Disease characteristics } \\
\hline ENSAT I, patients N(\%) & $2(4.2 \%)$ & & \\
\hline ENSAT II, patients N(\%) & $19(39.6 \%)$ & & \\
\hline ENSAT III, patients N(\%) & $10(20.8 \%)$ & & \\
\hline ENSAT IV, patients N(\%) & $17(35.4 \%)$ & & \\
\hline \multicolumn{4}{|l|}{ Target reaching characteristics } \\
\hline Patients reached the target $(\mathrm{N})$ & 41 & & \\
\hline 150 days $(N(\%))$ & $16(39.0 \%)$ & & \\
\hline 90 days $(\mathrm{N}(\%))$ & $19(46.3 \%)$ & & \\
\hline Target reaching time (days) & 142 & 113.9 & $24-579$ \\
\hline Duration of treatment (days) & 742 & 553.2 & $90-2856$ \\
\hline
\end{tabular}

SD, standard deviation; LBW, lean body weight; ASAT, aspartate transaminase; ALAT, alanine transaminase; GGT, gamma-glutamyl transferase; GFR, glomerular filtration rate; ENSAT, European Network for the Study of Adrenal Tumors ${ }^{a}$ at the start of treatment ${ }^{b}$ mean record of each patient 


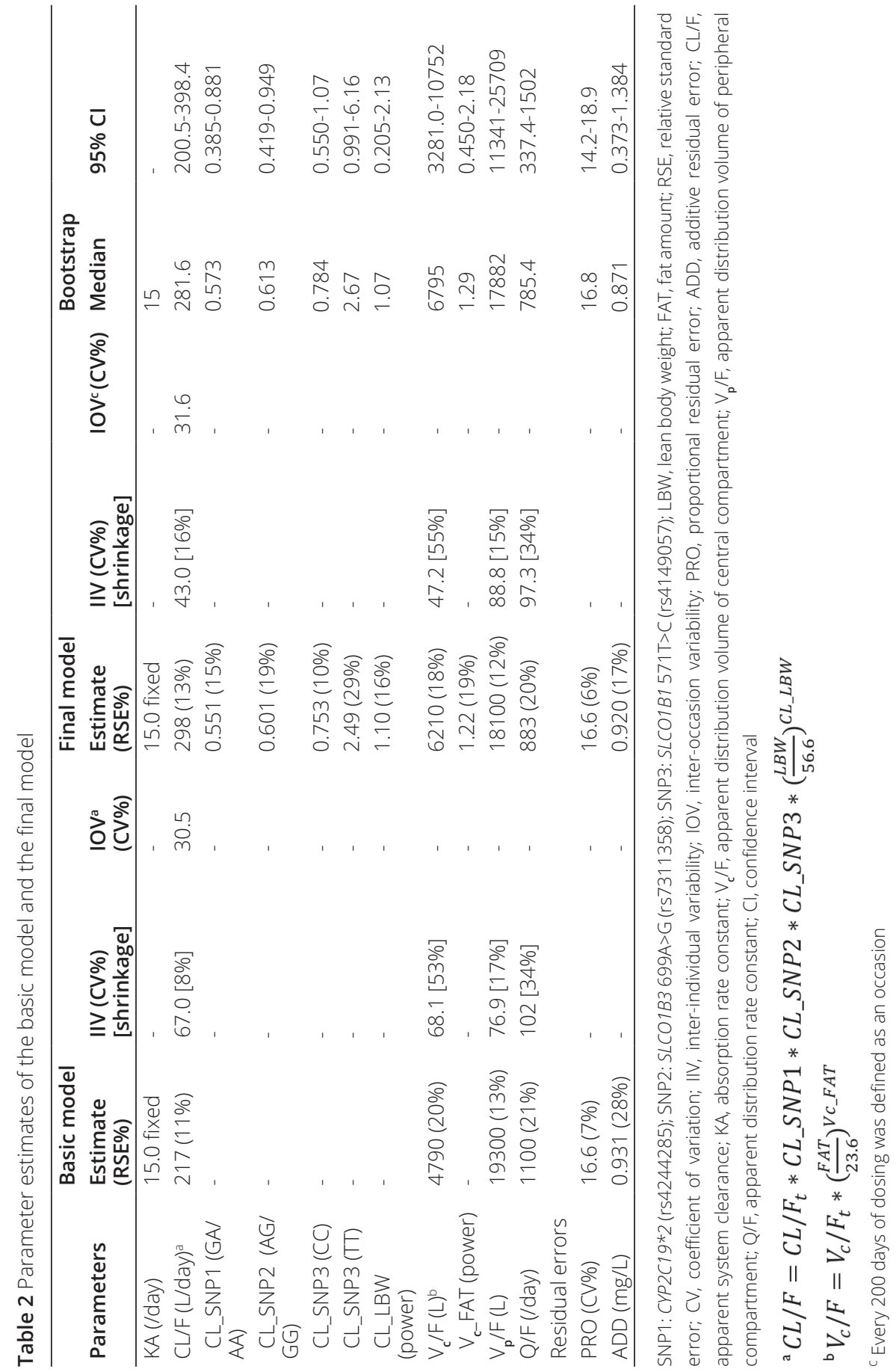


Table 3 Simulation results of different treatment regimens for included patients who originally reached the target $(\mathrm{N}=41)$

\begin{tabular}{|c|c|c|c|c|c|c|c|}
\hline $\begin{array}{l}\text { Regimen } \\
\text { (Fig. 1) }\end{array}$ & Mean & Max & Mean & Mean & $\begin{array}{l}\text { Median max } \\
/ \text { min } C_{\text {sim real }}\end{array}$ & $\begin{array}{l}\text { Starting dose } \\
\text { range (g) }\end{array}$ & \\
\hline 1 & 54.22 & 125 & 23.6 & 18.35 & $22.3 / 13.11$ & - & \\
\hline $2-2 g$ & 133.98 & 236 & 4.16 & 12.6 & $20.65 / 13.14$ & 2 & \\
\hline $2-4 g$ & 89.8 & 182 & 7.01 & 13.15 & $20.90 / 13.20$ & 4 & * \\
\hline $2-6 g$ & 60.61 & 149 & 13.85 & 15.13 & $21.13 / 13.09$ & 6 & \\
\hline 3-77day & 73 & 173 & 10.63 & 12.7 & $21.07 / 13.29$ & $3.5-7$ & \\
\hline 3-98day & 85.07 & 182 & 9.26 & 14.35 & $21.03 / 13.16$ & $3-6$ & * \\
\hline 3-119day & 97.9 & 191 & 6.44 & 12.22 & $20.96 / 13.21$ & $2.5-5$ & \\
\hline $4-(-4 g)$ & 89.8 & 182 & 5.96 & 12.66 & $20.91 / 13.22$ & 4 & * \\
\hline $4-50 \%$ & 89.8 & 182 & 8.82 & 12.37 & $20.91 / 13.22$ & 4 & * \\
\hline $5-(-4 g)$ & 85.07 & 182 & 7.92 & 13.01 & 20.84 / 13.14 & $3-6$ & * \\
\hline $5-50 \%$ & 85.07 & 182 & 11.13 & 12.21 & 20.84 / 13.22 & $3-6$ & \\
\hline $6-1$ & 91.12 & 194 & 6.61 & 13.37 & 20.84 / 12.91 & 4 & \\
\hline $6-2$ & 74.32 & 151 & 14.34 & 16.26 & $21.57 / 13.02$ & 4 & \\
\hline $7-1$ & 86.12 & 194 & 8.52 & 14.69 & $21.03 / 12.96$ & $3-6$ & * \\
\hline $7-2$ & 80.27 & 160 & 14 & 15.53 & $21.46 / 12.87$ & $2.5-5$ & \\
\hline 8 & 87.85 & 191 & 5.05 & 11.26 & 20.34 / 13.30 & $3.5-7$ & * \\
\hline 9 & 87.8 & 161 & 5.56 & 10.72 & $20.33 / 13.09$ & $3-10$ & * \\
\hline
\end{tabular}

$T_{\text {target }}$ target reaching time (the day when simulated mitotane concentration $14 \mathrm{mg} / \mathrm{L}$ ); $P_{\text {toxicity }}$ percentage of days when simulated mitotane concentrations were higher than the upper limit of mitotane therapeutic window $(20 \mathrm{mg} / \mathrm{L})$ in the first 200 days; $P_{\text {owndow' }}$ percentages of simulated mitotane concentrations located outside the therapeutic window after reaching the target. *Follow the optimization target 

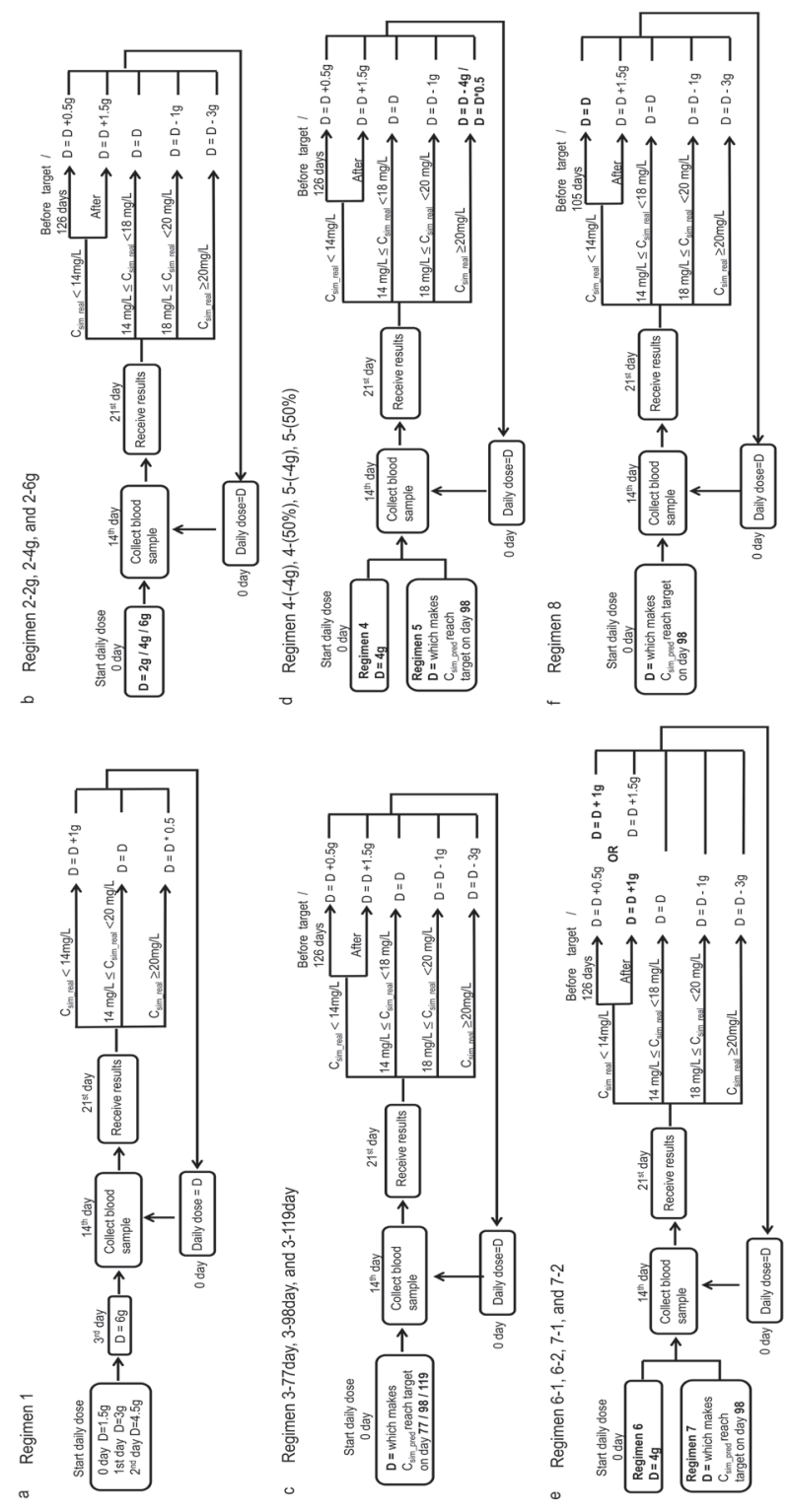

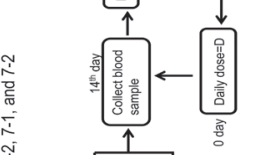
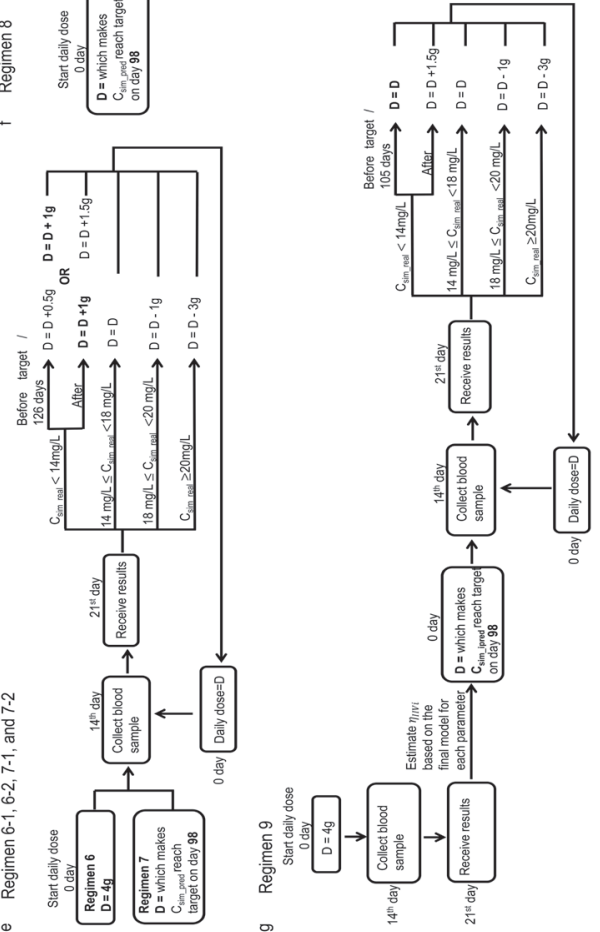

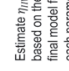

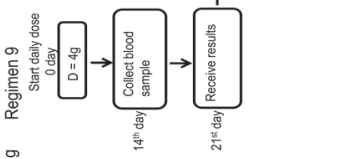


Fig. 1. Fig. 1. Designed treatment regimens that were evaluated by simulation: a) A previously reported dosing regimen (Regimen 1), where the dose started as $1.5 \mathrm{~g}$ per day and increased up to $6 \mathrm{~g}$ per day in 4 days and continued until next dose adjustment. The dosage was adjusted each time according to the monitored mitotane concentration level; b) Regimens where all patients started with $2 \mathrm{~g}$ (Regimen 2-2g), $4 \mathrm{~g}$ (Regimen 2-4g) or $6 \mathrm{~g}$ (Regimen 2-6g) per day. Dosage increased by $0.5 \mathrm{~g}$ every 21 days till the target was reached or 126 days if Csim_real $<14 \mathrm{mg} / \mathrm{L}$. Thereafter, the dosage increased by $1.5 \mathrm{~g}$ if Csim_real $<14 \mathrm{mg} / \mathrm{L}$, remained unchanged if $14 \mathrm{mg} / \mathrm{L} \leq \mathrm{Csim} \_$real $<18 \mathrm{mg} / \mathrm{L}$, decreased by $1 \mathrm{~g}$ if $18 \mathrm{mg} / \mathrm{L} \leq \mathrm{Csim} \_$real $<20 \mathrm{mg} / \mathrm{L}$, and decreased by $3 \mathrm{~g}$ if Csim_real $\geq 20 \mathrm{mg} / \mathrm{L}$; C) Regimens where patients started with individualized dose which allowed Csim_pred on day 77 (Regimen 3-77day), 98 (Regimen 3-98day), or 119 (Regimen 3-119day) reach the target. The rest dose adjustment strategies were same as Regimen 2; d) Regimens where patients started with $4 \mathrm{~g}$ per day (Regimen 4) or individualized dose (Regimen 5) and the dosage decreased by 4 g or 50\% if Csim_real $\geq 20$ $\mathrm{mg} / \mathrm{L}$. The rest dose adjustment strategies were same as Regimen 2; e) Regimens where patients started with $4 \mathrm{~g}$ per day (Regimen 6) or individualized dose (Regimen 7) and dosage increased by $1 \mathrm{~g}$ after reaching target or 126 days if Csim_real < 14mg/L (Regimen 6-1 and 7-1), or increased by $1 \mathrm{~g}$ till reaching target or 126 days if Csim_real < 14mg/L (Regimen 6-2 and 7-2). The rest dose adjustment strategies were same as Regimen 2; f) A regimen where patients started with individualized dose which remained unchanged till reaching target or 105 days if Csim_real < 14mg/L. The rest dose adjustment strategies was same as Regimen 2; g) A regimen where patients started with $4 \mathrm{~g}$ per day for the first 21 days and the next dosage was determined as which allowed Csim_ipred on day 98 reach the target (Regimen 9). The rest dose adjustment strategies was same as Regimen 8. Csim_real, simulated "real" mitotane concentrations based on individual parameters; Csim_pred, model predictions based on patients characteristics; Csim_ipred, the model predictions considering the interindividual variability ( $\left.\eta \_I I V i\right)$ estimated based on the first monitored concentration. 


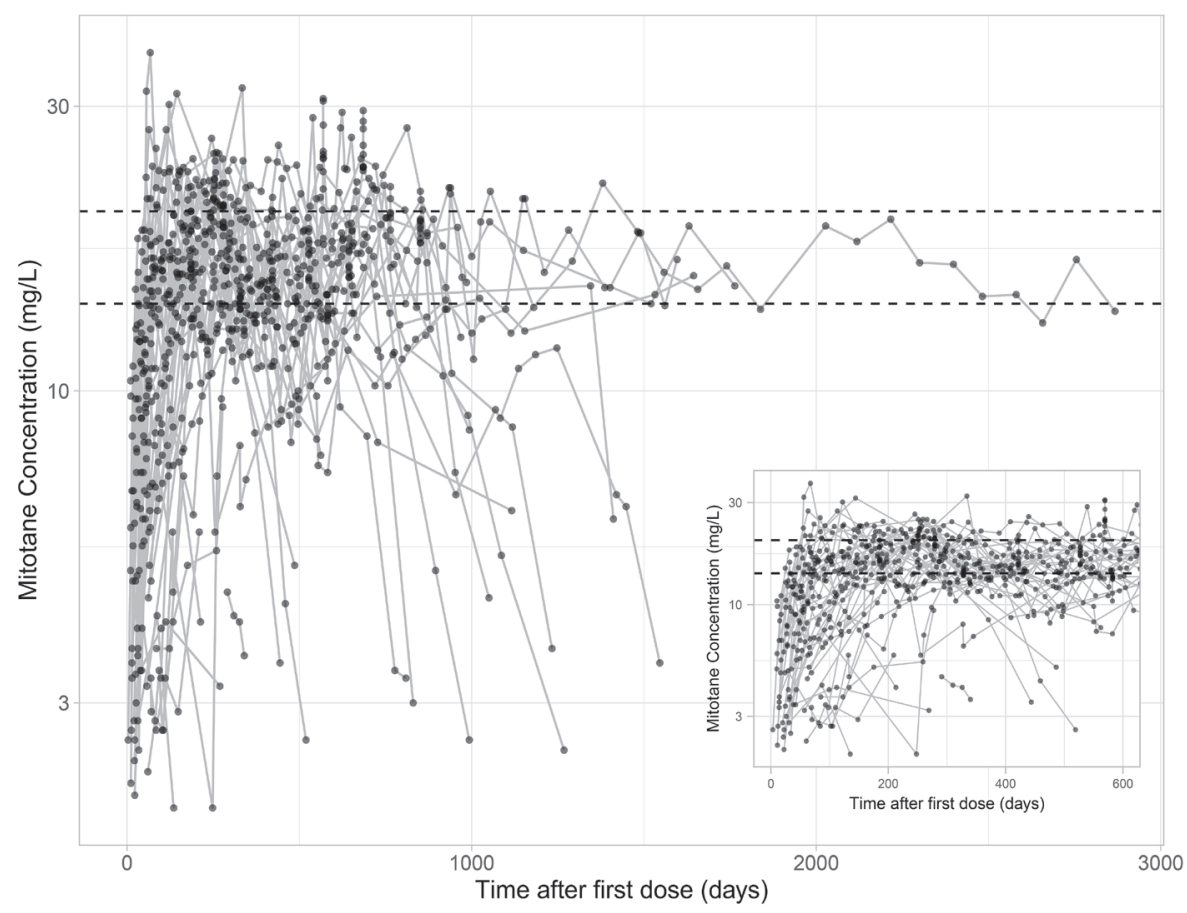

Fig. 2. Mitotane concentration-time curve collected from patients on logarithmic scale. Inserts show the data during the first 600 days of treatment. 


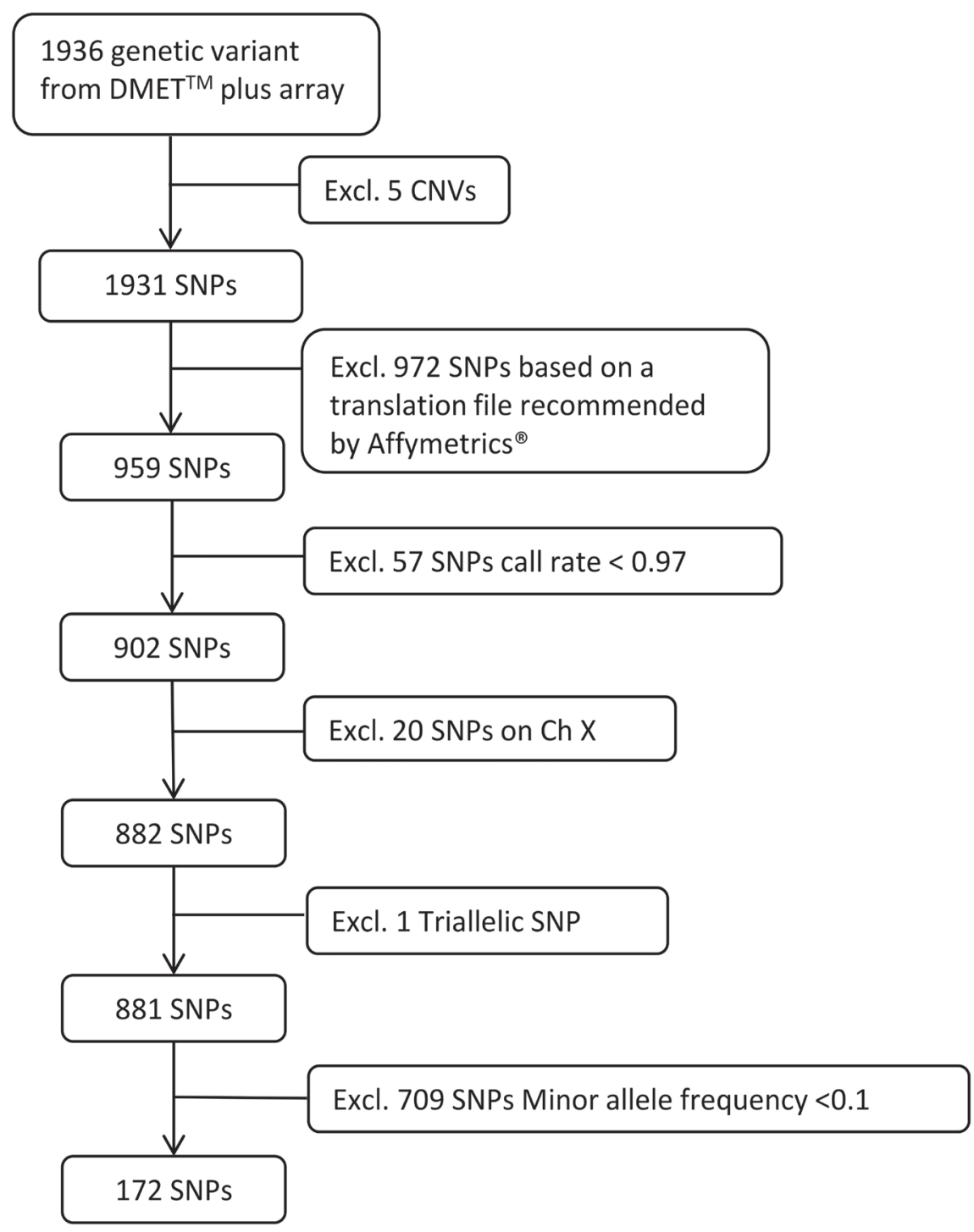

Fig. 3. Flow diagram of genetic variants selection. Excl. represents excluding, Ch X represents chromosome $\mathrm{X}$, DMETTM represent Drug Metabolizing Enzymes and Transporters, CNVs represents copy number variations. 

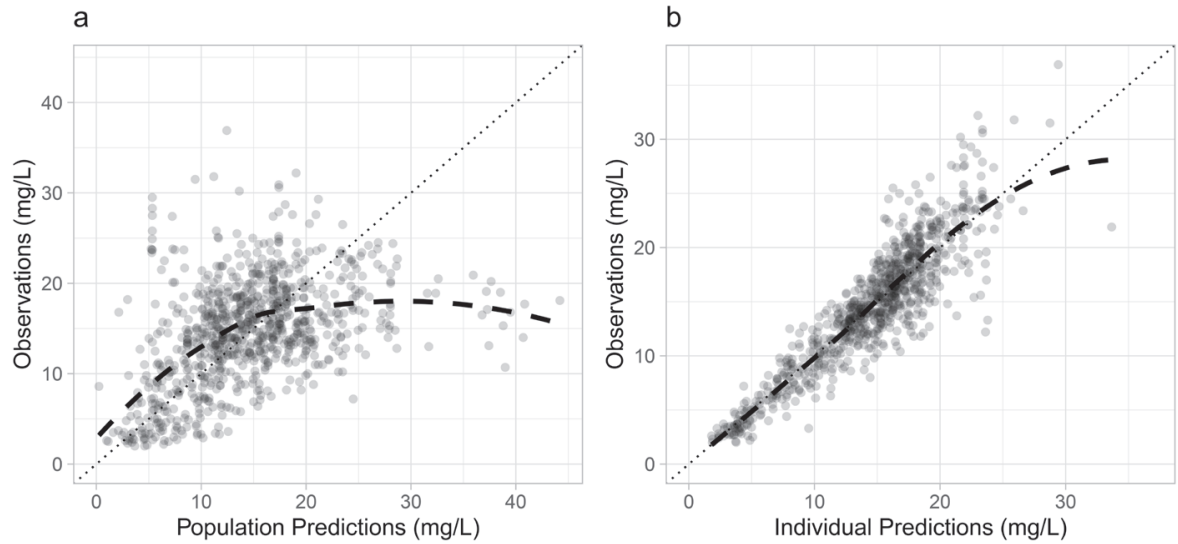

\section{C}
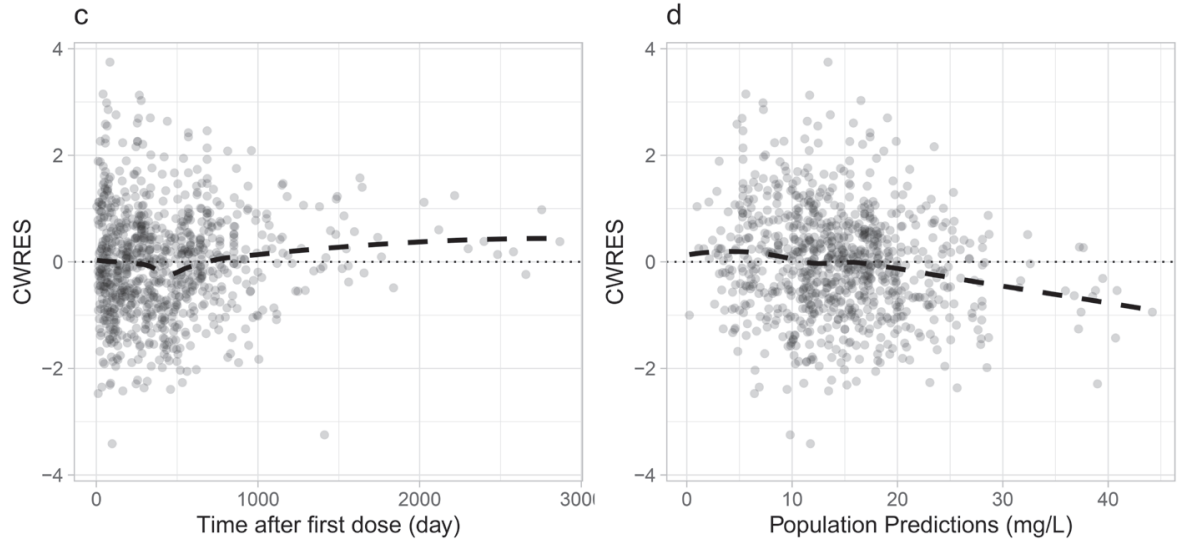

Fig. 4. Goodness-of-fit plots of the final population PK model of mitotane in patients with ACC, including observations versus population predictions (a) and individual predictions (b), and conditional weighted residual errors (CWRES) versus time $(c)$ and populations predictions $(d)$. The black dotted lines represent $y=x$ $(a, b)$ and $y=0$ (c, d). Black dashed lines represent corresponding loess regressions. 


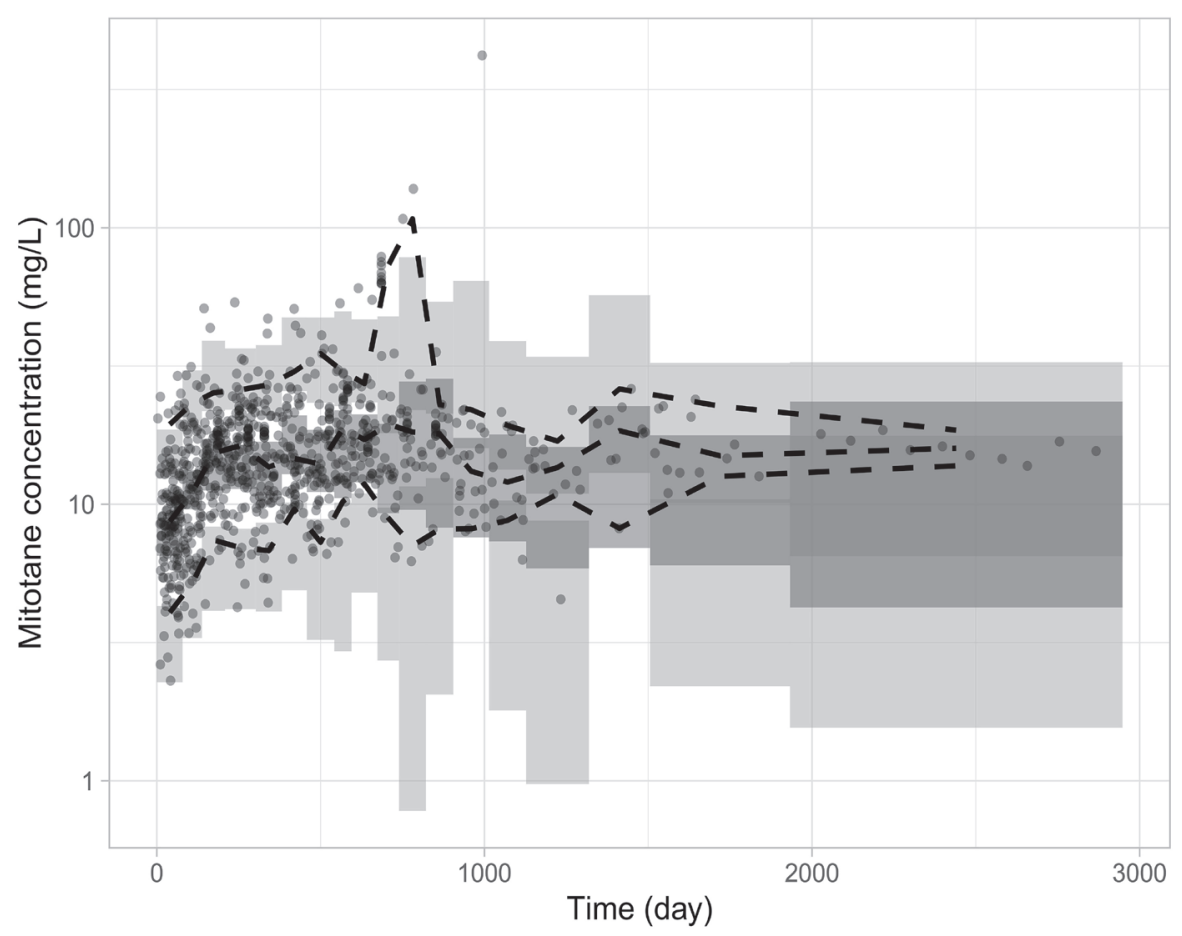

Fig. 5. Prediction corrected visual predictive check ( $p c \vee P C)$ plot of the final model on logarithmic scale. Black dashed lines represent 50th, 95th and 5th percentile of the observations, light grey shading areas represent $95 \%$ confidence interval of the 95 th and 5 th percentiles of the simulations respectively, and dark grey shading area represents $95 \%$ confidence interval of the 50 th percentiles of the simulations.

\section{Supplementary material caption}

1. Online Resource 1 Supplementary methods, figures and tables.

2. Online Resource 2 Shiny app establishing method and results

3. Online Resource 3 List of 959 SNPs from DMET ${ }^{\mathrm{TM}}$ array of which the genotyping results were obtained for each patient 


\section{References}

1. Paragliola RM, Torino F, Papi G, Locantore P, Pontecorvi A, Corsello SM. Role of Mitotane in Adrenocortical Carcinoma - Review and State of the art. Eur Endocrinol. 2018;14(2):62-6. doi:10.17925/EE.2018.14.2.62.

2. Moolenaar AJ, van Slooten $H$, van Seters AP, Smeenk D. Blood levels of o, $\mathrm{p}^{\prime}-\mathrm{DDD}$ following administration in various vehicles after a single dose and during long-term treatment. Cancer Chemother Pharmacol. 1981;7(1):51-4. doi:10.1007/bf00258213.

3. Arshad U, Taubert M, Kurlbaum M, Frechen S, Herterich S, Megerle Fet al. Enzyme autoinduction by mitotane supported by population pharmacokinetic modelling in a large cohort of adrenocortical carcinoma patients. Eur J Endocrinol. 2018;179(5):287-97. doi:10.1530/EJE-18-0342.

4. Vanslooten H, Vanseters AP, Smeenk D, Moolenaar AJ. O,P'-Ddd (Mitotane) Levels in Plasma and Tissues during Chemotherapy and at Autopsy. Cancer Chemoth Pharm. 1982;9(2):85-8. doi:10.1007/bf00265384.

5. Kerkhofs TM, Baudin E, Terzolo M, Allolio B, Chadarevian R, Mueller HH et al. Comparison of two mitotane starting dose regimens in patients with advanced adrenocortical carcinoma. J Clin Endocrinol Metab. 2013;98(12):4759-67. doi:10.1210/jc.2013-2281.

6. Buil-Bruna N, Lopez-Picazo JM, Martin-Algarra S, Troconiz IF. Bringing Model-Based Prediction to Oncology Clinical Practice: A Review of Pharmacometrics Principles and Applications. Oncologist. 2016;21(2):220-32. doi:10.1634/theoncologist.2015-0322.

7. Cazaubon Y, Talineau Y, Feliu C, Konecki C, Russello J, Mathieu O et al. Population Pharmacokinetics Modelling and Simulation of Mitotane in Patients with Adrenocortical Carcinoma: An Individualized Dose Regimen to Target All Patients at Three Months? Pharmaceutics. 2019;11(11). doi:10.3390/ pharmaceutics11110566.

8. Kerkhofs TM, Derijks LJ, Ettaieb H, den Hartigh J, Neef K, Gelderblom H et al. Development of a pharmacokinetic model of mitotane: toward personalized dosing in adrenocortical carcinoma. Ther Drug Monit. 2015;37(1):58-65. doi:10.1097/FTD.0000000000000102.

9. Scripture CD, Sparreboom A, Figg WD. Modulation of cytochrome P450 activity: implications for cancer therapy. Lancet Oncol. 2005;6(10):780-9. doi:10.1016/S1470-2045(05)70388-0.

10. D'Avolio A, De Francia S, Basile V, Cusato J, De Martino F, Pirro E et al. Influence of the CYP2B6 polymorphism on the pharmacokinetics of mitotane. Pharmacogenet Genomics. 2013;23(6):293300. doi:10.1097/FPC.0b013e3283606cb2.

11. Mornar A, Sertic M, Turk N, Nigovic B, Korsic M. Simultaneous analysis of mitotane and its main metabolites in human blood and urine samples by SPE-HPLC technique. Biomed Chromatogr. 2012;26(11):1308-14. doi:10.1002/bmc.2696.

12. Hermsen IG, den Hartigh J, Haak HR. Mitotane serum level analysis; good agreement between two different assays. Clin Endocrinol (Oxf). 2010;73(2):271-2. doi:10.1111/j.1365-2265.2010.03787.x.

13. Boer $P$. Estimated lean body mass as an index for normalization of bodv fluid volumes in humans. American Journal of Physiology-Endocrinology and Metabolism. 1984;247(4):F632-6. doi:10.1152/ ajprenal.1984.247.4.F632.

14. Arbitrio M, Di Martino MT, Scionti F, Agapito G, Guzzi PH, Cannataro M et al. DMET (Drug Metabolism Enzymes and Transporters): a pharmacogenomic platform for precision medicine. Oncotarget. 2016;7(33):54028-50. doi:10.18632/oncotarget.9927.

15. Caldwell MD, Awad T, Johnson JA, Gage BF, Falkowski M, Gardina P et al. CYP4F2 genetic variant alters required warfarin dose. Blood. 2008;111(8):4106-12. doi:10.1182/blood-2007-11-122010.

16. Dumaual C, Miao X, Daly TM, Bruckner C, Njau R, Fu DJ et al. Comprehensive assessment of 
metabolic enzyme and transporter genes using the Affymetrix Targeted Genotyping System. Pharmacogenomics. 2007;8(3):293-305. doi:10.2217/14622416.8.3.293.

17. Affymetrix ${ }^{\circledR}$. White Paper: DMETTM Plus allele translation reports: Summary of comprehensive drug disposition genotyping into commonly recognized allele names 2012. http://tools.thermofisher. com/content/sfs/brochures/dmet plus translation.pdf. Accessed 06 Jan 2020.

18. Burmester JK, Sedova M, Shapero MH, Mansfield E. DMET microarray technology for pharmacogenomics-based personalized medicine. Methods Mol Biol. 2010;632:99-124. doi:10.1007/978-1-60761-663-4_7.

19. Sissung TM, English BC, Venzon D, Figg WD, Deeken JF. Clinical pharmacology and pharmacogenetics in a genomics era: the DMET platform. Pharmacogenomics. 2010;11(1):89103. doi:10.2217/pgs.09.154.

20. Deeken J. The Affymetrix DMET platform and pharmacogenetics in drug development. Curr Opin Mol Ther. 2009;11(3):260-8. PMID 19479659

21. Keizer RJ, Jansen RS, Rosing H, Thijssen B, Beijnen JH, Schellens JH et al. Incorporation of concentration data below the limit of quantification in population pharmacokinetic analyses. Pharmacol Res Perspect. 2015;3(2):e00131. doi:10.1002/prp2.131.

22. Hecht M, Veigure R, Couchman L, Cl SB, Standing JF, Takkis K et al. Utilization of data below the analytical limit of quantitation in pharmacokinetic analysis and modeling: promoting interdisciplinary debate. Bioanalysis. 2018;10(15):1229-48. doi:10.4155/bio-2018-0078.

23. Jonsson EN, Karlsson MO. Automated covariate model building within NONMEM. Pharm Res. 1998;15(9):1463-8. doi:10.1023/a:1011970125687.

24. Bergstrand M, Hooker AC, Wallin JE, Karlsson MO. Prediction-corrected visual predictive checks for diagnosing nonlinear mixed-effects models. AAPS J. 2011;13(2):143-51. doi:10.1208/s12248011-9255-z.

25. Wang W, Hallow KM, James DA. A Tutorial on RxODE: Simulating Differential Equation Pharmacometric Models in R. CPT Pharmacometrics Syst Pharmacol. 2016;5(1):3-10. doi:10.1002/ psp4.12052.

26. Koninklijke Nederlandse Maatschappij ter bevordering der Pharmacie. Mitotaan. 2019. https:// kennisbank.knmp.nl/article/Informatorium Medicamentorum/S1853.html. Accessed 28 Aug 2019.

27. Whirl-Carrillo M, McDonagh EM, HebertJM, Gong L, Sangkuhl K, Thorn CF et al. Pharmacogenomics knowledge for personalized medicine. Clin Pharmacol Ther. 2012;92(4):414-7. doi:10.1038/ clpt.2012.96.

28. PharmGKB. rs4244285, Variant annotation. https://www.pharmgkb.org/variant/PA166154053/ variantAnnotation. Accessed 28 Aug 2019

29. PharmGKB. rs7311358, Clinical annotation. https://www.pharmgkb.org/variant/PA166154602/ clinicalAnnotation. Accessed 28 Aug 2019.

30. PharmGKB. rs4149117, Clinical annotation. https://www.pharmgkb.org/variant/PA166154583/ clinicalAnnotation. Accessed 28 Aug 2019. 
Online Resource 1 Supplementary methods, figures and tables.

\section{Supplementary population PK analysis methods}

One-, two- and three-compartment models, with first-order absorption and firstorder elimination, were explored as the structural model. Relative standard error (RSE) of parameters, which represent the precision of parameter estimates, and the objective function value (OFV) were considered when evaluating the structural models. The one with acceptable RSE and lower OFV was selected as the final basic model structure.

Inter-individual variability (IIV) of parameters were estimated with Eq. 1 , where $P_{i}$ represents the parameter of $i$ th individual and was assumed to be log-normally distributed, $P_{t}$ represents typical value of the parameter, and $\eta_{I V}$ represents the random IIV which was assumed to be normally distributed with mean of 0 and variance of $\omega_{1}{ }^{2}$. In addition, inter-occasion variability (IOV), which reflects the intra-individual variability, of apparent systematic clearance (CL/F) was also included when analyzing the full dataset. As is shown in Eq.S1, $\eta_{I O V}$ represents the random IOV. The distribution of $\eta_{I O V}$ in each occasion was assumed to be similar and normally distributed with mean of 0 and variance of $\omega_{2}{ }^{2}$. In this study, every 200 days of treatment was defined as an occasion as the total observation periods of the patients were long.

The residual error was characterized with a combined proportional and additive model as is shown in Eq. S2, where Obs represents observations, IPRED represents individual predictions, and $\varepsilon_{1}$ and $\varepsilon_{2}$ represent the proportional residual error and additive residual error respectively which were assumed to be normally distributed with mean of 0 and variance of $\sigma_{1}{ }^{2}$ and $\sigma_{2}{ }^{2}$, respectively.

$P i=P t \cdot$ e $I I V i+\eta I O V j$

Eq.S1

$O b s=I P R E D \cdot\left(1+\varepsilon_{1}\right)+\varepsilon_{2}$

Eq.S2

As for the covariate analysis, the identified SNPs, as well as patients' demographic information and clinical characteristics were considered. For continuous covariates, for each patient the mean values of all measurements during the monitoring period were taken. In case of missing continuous covariates, the corresponding median value of all patients was assigned. For patients who only missed HT but not

WT, LBW was calculated using real WT and imputed HT. For GFR, 0 (normal) was assigned if $\geq 50 \%$ of the collected patient's records were 0 otherwise 1 was assigned. Patients who missed GFR measurements, 0 was assigned.

The effect of all above covariates on mitotane CL/F and the effect of WT, LBW, FAT, 
and gender on apparent distribution volumes (V/F) were investigated using stepwise covariate modelling (SCM) function implemented with Perl-Speaks-NONMEM (version 4.7.0) $)^{1}$. Both a forward inclusion $(p<$

0.05) and a backward elimination process ( $p$ < 0.01) were performed to identify significant covariates. For SNPS that were in 100\% linkage disequilibrium, if they were included during the SCM analysis, the more clinically relevant ones would be selected in the final model. The effects of continuous covariates were investigated with both linear relation (Eq.S3) and power relation (Eq.S4), where $P_{i}$ represents the parameter of $i$ th individual, $P_{t}$ represents typical value of the parameter, and $\eta_{i}$ represents the individual variability, $\theta_{\text {cov }}$ represents the estimate of covariate effect, $\mathrm{COV}_{i}$ represents the covariate value of $i$ th individual, $\mathrm{COV}_{m}$ is the median value of the covariate. Categorical covariates were analyzed with Eq. S5, where $\theta_{\text {cov }}$ was set as 1 for reference category and was estimated for other categories.

$$
\begin{array}{lr}
P_{i}=P_{t} \cdot\left(1 \pm \theta_{\operatorname{COV}} \cdot\left(\operatorname{COV}_{i}-\operatorname{COV}_{m}\right)\right) \cdot \mathrm{e}^{\eta i} & \text { Eq.S3 } \\
\operatorname{COVi} \theta \operatorname{COV} \cdot \mathrm{e} \eta i & \text { Eq.S4 } \\
P i=P t \cdot(\operatorname{COVm}) & \\
P_{i}=P_{t} \cdot \theta_{\operatorname{CoV}} \cdot \mathrm{e}^{\eta i} & \text { Eq.S5 }
\end{array}
$$

\section{Supplementary model evaluation methods}

pcVPC was performed by 1000 times of simulation and the data points, $5^{\text {th }}, 50^{\text {th }}$, and 95 $5^{\text {th }}$ percentiles of prediction-corrected observations were plotted together with 95\% confidence intervals $(\mathrm{Cl})$ of $5^{\text {th }}$,

$50^{\text {th }}$, and $95^{\text {th }}$ percentiles of simulations. NPDE evaluation was performed with npde package (version 2.0) implemented in R statistics software based on 1000 times of simulations. The bootstrap was conducted by 1000 runs of bootstrap replicates sampled from original dataset with replacement, which was stratified on whether the subject contributed more than two data points after the end of treatment.

The median as well as 95\% Cl of parameters were derived and compared with original parameter estimates.

\section{Supplementary simulation method}

Based on the final model structure, simulations were performed to evaluate different designed treatment strategies and approaches of starting dose determination. Patients were assumed to receive treatment as long as their last mitotane 
concentration monitoring time. The blood samples were assumed to be collected once every 2 weeks after knowing the result of the last sample, and the concentration of mitotane was assumed to be known 7 days after blood collection, which is in accordance with the optimal scenario in the clinical practice. The dose amount was subsequently adjusted accordingly.

As a comparison, a previous recommended 'high-dose' starting regimens, where the mitotane dose starts with $1.5 \mathrm{~g}$ per day and increases up to $6 \mathrm{~g}$ per day in 4 days, were simulated (Regimen 1$)^{2}$.

As for the newly designed regimens, the starting dose was 1) set as $2 \mathrm{~g}$, $4 \mathrm{~g}$, or $6 \mathrm{~g}$ for all patients according to the guideline ${ }^{3}$ (Regimen 2, 4, and 6) or 2) set individually considering patients characteristics with the help of the model (Regimen 3, 5, 7, and 8). As the expected time to reach the therapeutic target of mitotane is 3 to 5 months, the individually starting daily mitotane dose was estimated as the dose that allows the predicted mitotane concentrations on day $98\left(C_{\text {sim_pred98 }}\right)$ reach the therapeutic target. The $C_{\text {sim_pred98 }}$ was obtained by performing simulation under a regimen of $6 \mathrm{~g}$ per day increasing by $0 \mathrm{~g}$ (Regimen 8), $0.5 \mathrm{~g}$ (Regimen 2, 3, 4, 5, 6-1, and 7-1), or $1 \mathrm{~g}$ (Regimen 6-2 and 7-2) once every 21 days till the $98^{\text {th }}$ day of treatment, with only typical parameter values and covariate effects considered. Given the linear PK feature of mitotane, the suggested starting daily dose (Dose) was therefore determined by Eqs. S6 and S7, where $\lceil X\rceil$ represents the least integer greater than or equal to

$X,\lfloor X\rfloor$ represents the greatest integer less than or equal to $X$. Determining the starting dose based on the $C_{\text {sim_pred }}$ on day 77 and 119 were also used for comparison.

$$
X=\frac{14 \mathrm{mg} / \mathrm{L}}{{ }^{c}{ }_{\text {sim_im_pred }}} \cdot 6 \mathrm{~g}
$$$$
[X\rceil, \quad X-[X]>0.650 \text { Dose }=\{\lfloor X\rfloor+0.5, \quad 0.350 \leq X-\lfloor X\rfloor \leq 0.650
$$$$
\text { Eq. S7 }\lfloor X\rfloor, \quad X-\lfloor X\rfloor<0.350
$$

Besides the above regimens, since individual parameters could be estimated after knowing one TDM result, Regimen 9 was also designed and evaluated. In this strategy, patients were assumed to start with $4 \mathrm{~g}$ per day until the first TDM result was obtained. $C_{\text {sim_real }}$ of each patient on day 14 was simulated, based on which the $\eta_{I I V i}$ and $\eta_{I O V 1}$ were estimated for each patient using NONMEM with the POSTHOC function. Subsequently, the next daily dose of each patient was determined with Eq. S6-S7 according to the individual $C_{\text {sim_pred98 }}\left(C_{\text {sim_ipred98 }}\right)$ under the daily dosing of $6 \mathrm{~g}$, based on the model incorporating $\eta_{I I V i}$ as was suggested in a previous study ${ }^{4}$. The constant starting regimen was applied in this regimen. 
In Regimen 2 to 8 , the dose increasing amount when $C_{\text {sim_real }}<14 \mathrm{mg} / \mathrm{L}$ was set differently before and after the target was reached (starting and maintenance regimen), in order to limit the toxicity at start and maintain the mitotane trough concentration within the therapeutic range at a later phase. The combination of $0 \mathrm{~g} / 1.5 \mathrm{~g}, 0.5 \mathrm{~g} / 1.5 \mathrm{~g}, 0.5 \mathrm{~g} / 1 \mathrm{~g}$, and $1 \mathrm{~g} / 1.5 \mathrm{~g}$ were simulated and evaluated. Regimen 2 to 7 applied stepwise increasing starting regimen and Regimen 8 applied constant starting regimen. A maximum number of days that follows the starting regimen was set as 126 (around 4 months) and 105 (98+7 days) for the stepwise increasing or constant starting regimens, respectively.

When $C_{\text {sim_real }}$ reached $20 \mathrm{mg} / \mathrm{L}$, a 50\% dose reduction was suggested in Regimen 1. In comparison, both fixed dose amount reduction ( $3 \mathrm{~g}$ or $4 \mathrm{~g}$ ) and $50 \%$ reduction were evaluated in the newly designed regimens (Regimen 2 to 9). If a reduction resulted in a dose level lower than $0 \mathrm{~g}$, then $0 \mathrm{~g}$ was applied. Besides, an additional concentration threshold of dose reduction, $18 \mathrm{mg} / \mathrm{L}$, with $1 \mathrm{~g}$ dose reduction was introduced in Regimen 2 to 9, since a 7-day period of no dose adjustment presented.

\section{Reference}

1. Jonsson, E.N. \& Karlsson, M.O. Automated covariate model building within NONMEM. Pharmaceut Res 15, 1463-8 (1998).

2. Kerkhofs, T.M. et al. Comparison of Two Mitotane Starting Dose Regimens in Patients With Advanced Adrenocortical Carcinoma. The Journal of Clinical Endocrinology \& Metabolism 98, 4759 67 (2013).

3. Koninklijke Nederlandse Maatschappij ter bevordering der Pharmacie. Mitotaan. <https://kennisbank.knmp.n//article/Informatorium Medicamentorum/S1853.html> Accessed 28 Aug 2019.

4. Abrantes, J.A., Jönsson, S., Karlsson, M.O. \& Nielsen, E.I. Handling interoccasion variability in model-based dose individualization using therapeutic drug monitoring data. British journal of clinical pharmacology 85, 1326-36 (2019). 
Table S1 Potential SNPs out of the 959 SNPs that are correlated to mitotane clearance based on the association analysis.

Table S2 Additional potential SNPs that are correlated to mitotane clearance based on the association analysis, if the pre-set selection based on a translation file as recommended bybAffymetrics ${ }^{\circledR}$ was not considered.

Fig. S1. The population PK model structure of mitotane.

Fig. S2 The boxplots of estimated $\eta I I V i_{-} C L$ in each genotype group of SNP (a) CYP2C19*2 (rs4244285), (b) SLCO1B3 699A>G (rs7311358), and (c) SLCO1B1 571T>C (rs4149057).

Fig. S3. Normalized prediction distribution error (NPDE) results of the final population PK model of mitotane in patients with ACC.

Fig. S4. The estimates of inter-occasion variability (IOV) over time. Red dashed lines represent loess regression result.

Fig. S5 Flow diagram of the genetic variants selection if the pre-set selection based on a translation file as recommended by Affymetrics ${ }^{\circledR}$ was not considered.

Table S1 Potential SNPS out of the 959 SNPs that are correlated to mitotane clearance based on the association analysis

\begin{tabular}{lllll}
\hline Gene & & Common Name & dbSNP.RS.ID & P value \\
\hline 1 & CYP2C18 & CYP2C18_C.1154C>T(T385M) & rs2281891 & 0.020 \\
2 & CYP2C19 & CYP2C19*2_19154G>A(P227P) & rs4244285 & 0.020 \\
3 & SLCO1B3 & SLCO1B3_C.334G>T(A112S) & rs4149117 & 0.027 \\
4 & SLCO1B3 & SLCO1B3_C.699A>G(I233M) & rs7311358 & 0.027 \\
5 & SLCO1B3 & SLCO1B3_C.1557G>A(A519A) & rs2053098 & 0.027 \\
6 & SLCO1B1 & SLCO1B1_C.571T>C(L191L) & rs4149057 & 0.020 \\
7 & VKORC1 & VKORC1_C.*134G>A(3'UTR) & rs7294 & 0.050 \\
8 & VKORC1 & VKORC1_C.283+124G>C & rs8050894 & 0.030 \\
9 & VKORC1 & VKORC1_C.174-136C>T & rs9934438 & 0.030 \\
10 & VKORC1 & VKORC1_C.- & rs9923231 & 0.030 \\
& & 1639G>A(Promoter) & & \\
11 & UGT1A6 & UGT1A6_C.315A>G(L105L) & rs1105880 & 0.042 \\
\hline
\end{tabular}


Table S2 Additional potential SNPs that are correlated to mitotane clearance based on the association analysis, if the pre-set selection based on a translation file as recommended by Affymetrics ${ }^{\circledR}$ was not considered.

\begin{tabular}{lllll}
\hline Gene & & Common Name & dbSNP.RS.ID & P value \\
\hline 1 & CA5P & CA5P_A>G(rS11859842) & rs11859842 & 0.029 \\
2 & SLC16A1 & SLC16A1_C.*1942T>C & rs9429505 & 0.0067 \\
3 & CHST10 & CHST10_C.*381G>A & rs1530031 & 0.040 \\
4 & CYP20A1 & CYP20A1_50767C>T(L346F) & rs1048013 & 0.014 \\
5 & SLC22A13 & SLC22A13_C.*8336G>A & rs4679028 & 0.032 \\
6 & UGT2A1 & UGT2A1_C.1305-109A>C & rs2288741 & 0.042 \\
7 & ADH6 & ADH6_C.-930T>C & rs10002894 & 0.012 \\
8 & ADH6 & ADH6_C.-2874T>C & rs6830685 & 0.012 \\
9 & SLCO5A1 & SLCO5A1_C.97C>T(L33F) & rs3750266 & 0.015 \\
\hline
\end{tabular}

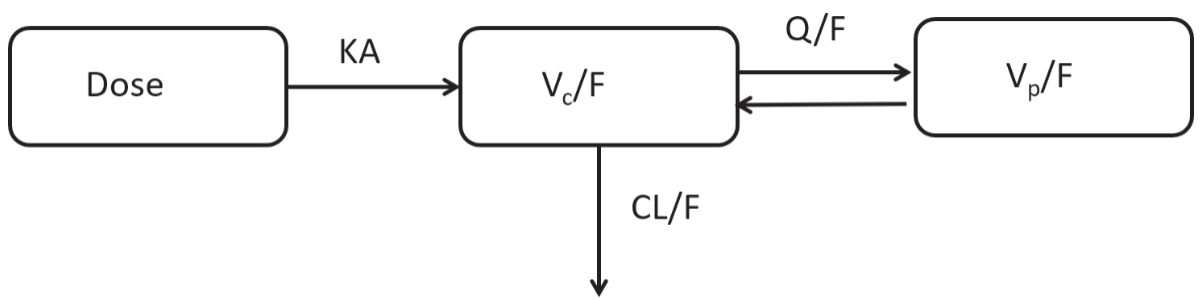

Fig. S1. The population PK model structure of mitotane. CL/F represents apparent system clearance, KA represents absorption rate constant, VC/F represents apparent distribution volume of central compartment, $\mathrm{Vp} / \mathrm{F}$ represents apparent distribution volume of peripheral compartment, Q/F represents apparent distribution rate constant.
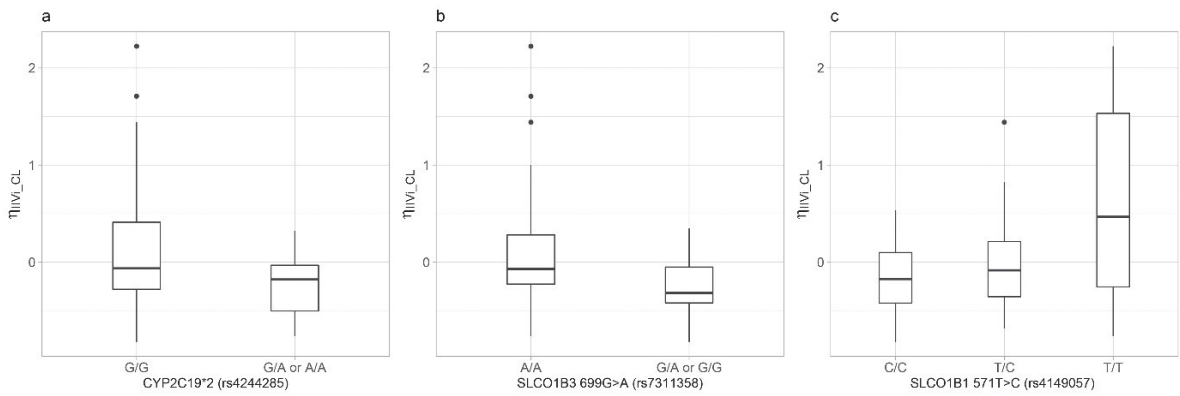

Fig. S2 The boxplots of estimated $\eta_{I V i_{-} C L}$ in each genotype group of SNP (a) CYP2C19*2 (rS4244285), (b) SLCO1B3 699A>G (rs7311358), and (c) SLCO1B1 571T>C (rs4149057) 

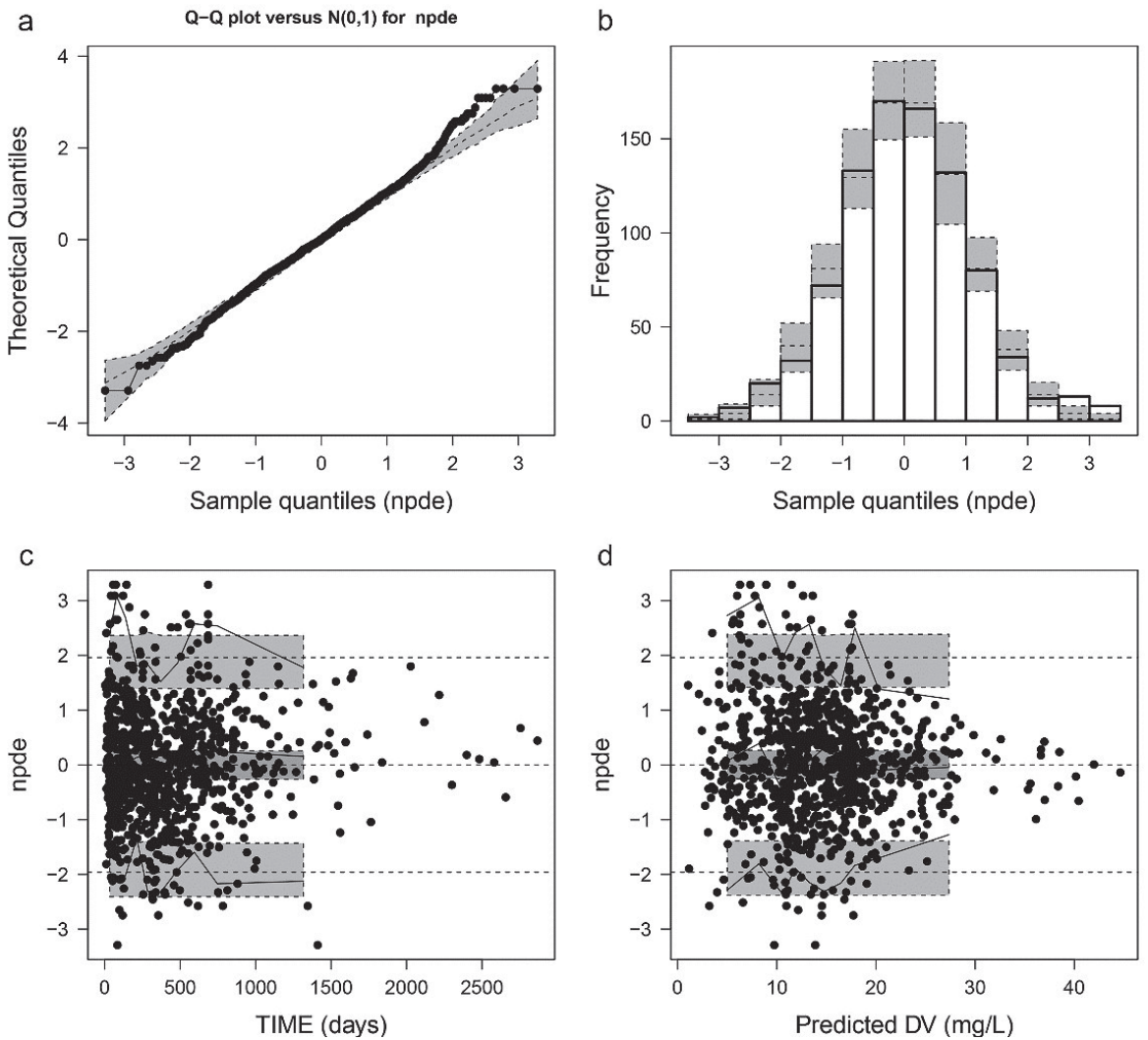

Fig. S3. Normalized prediction distribution error (NPDE) results of the final population PK model of mitotane in patients with ACC, including the quantile-quantile plot (a), the distribution histogram of NPDE (b), and the NPDE versus time (c) and population predictions (d). The NPDE results are shown to distribute around a mean of 0.03616 with a variance of 1.134 . 


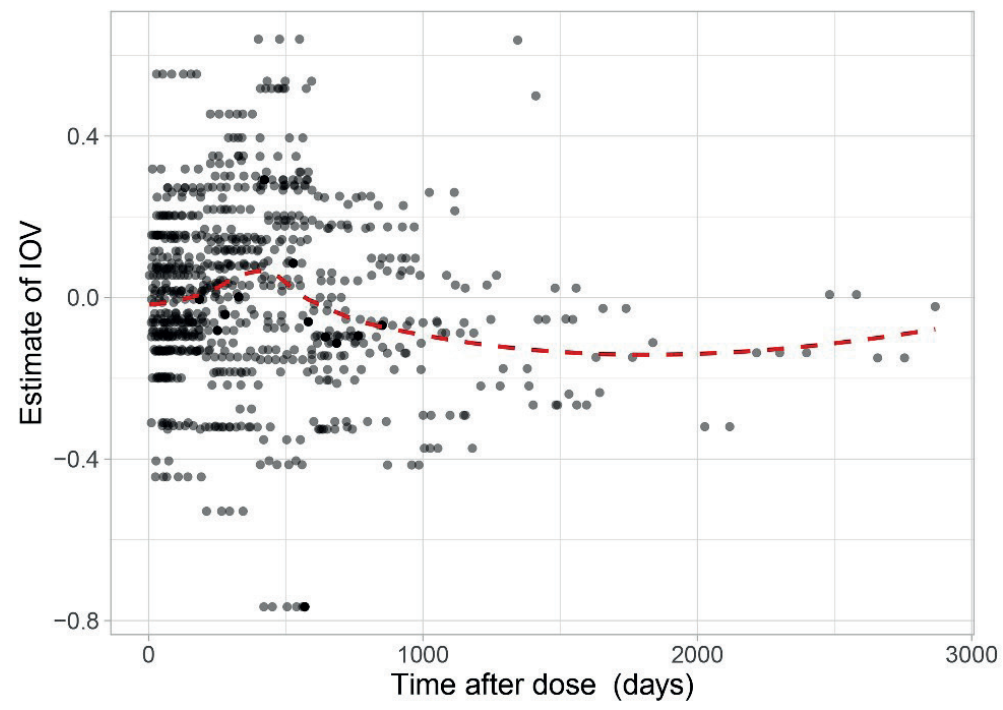

4

Fig. S4. The estimates of inter-occasion variability (IOV) over time. Red dashed lines represent loess regression result. 


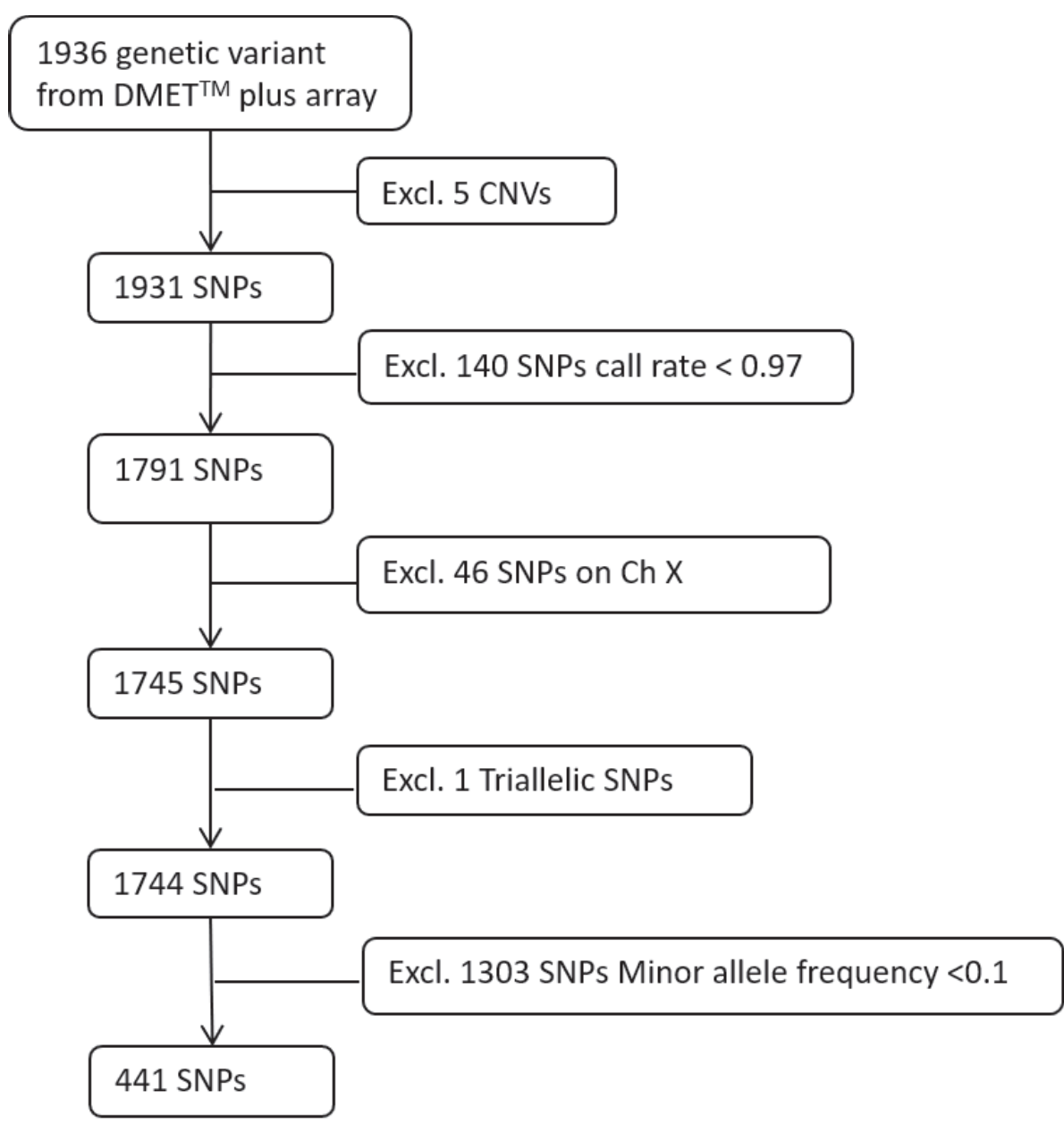

Fig. S5 Flow diagram of the genetic variants selection if the pre-set selection based on a translation file as recommended by Affymetrics ${ }^{\circledR}$ was not considered. Excl. represents excluding, Ch X represents chromosome X, DMET ${ }^{\mathrm{TM}}$ represent Drug Metabolizing Enzymes and Transporters, CNVs represents copy number variations. 
Online Resource 2 Shiny app establishing method and results

\author{
Shiny app establishment
}

Based on the final mitotane population pharmacokinetic model, a Shiny app was established for the simulation for a random patient and to elucidate an example of the model application on guiding treatment for a new patient. Package shiny (version 1.4.0) and RxODE (version 0.6-1) in R statistics software (version 3.4.2; R Foundation for Statistical Computing, Vienna, Austria) were utilized. The R script can be found through: https://github.com/AnyueYin/Shiny-app-script-for-model-simulation-Population-PK-and-PG-analysis-of-mitotane. Patient gender, weight, and height, which were used to estimate lean body weight (LBW) and fat amount (FAT), as well as the results of three SNPs were in the input panel, based on which the starting dose was suggested. One hundred times of simulation under an optimized mitotane treatment regimen, Regimen 5-(-4g), were performed given the input information. The $90 \%$ prediction interval, $50^{\text {th }}$ percentile of the predictions, target reaching time, and suggested starting dose were plotted in the output figure. The residual errors were not considered in the simulation.

Screen shots of the developed shiny app is shown in Fig. S6. The result shows that for a male patient with $85 \mathrm{~kg}$ weight and $180 \mathrm{~cm}$ height who carries G/G, A/A, and T/C for CYP2C19*2 (rs4244285), SLCO1B3 699A>G (rs7311358), and SLCO1B1 $571 \mathrm{~T}>\mathrm{C}$ (rs4149057), respectively, the $90 \%$ prediction interval can nicely locate within the therapeutic window of mitotane. The starting dose was suggested as $5.5 \mathrm{~g}$ per day and the $50^{\text {th }}$ percentile of the predictions reached the target on day 92 . If the genotype result of CYP2C19*2 (rs4244285) changed to G/A, the suggested starting dose became $4 \mathrm{~g}$ per day and the $50^{\text {th }}$ percentile of the predictions reached the target on day 94 .

In addition, a model with FAT effect on central distribution volume as the only covariate (Table S3) was also built in the Shiny app as an alternative option to allow dosing advice and concentration prediction for patients when genotyping results are not available (Figure S6c). 
Table S3 Parameter estimates of the final model without genotyping results as covariates

\begin{tabular}{llll}
\hline Parameters & Final model & & \\
& Estimate (RSE\%) & IIV (CV\%) [shrinkage] & IOVa (CV\%) \\
\hline KA (/day) & 15.0 fixed & - & - \\
CL/F (L/day) & $217(9 \%)$ & $66.3[7 \%]$ & 31.2 \\
$V_{c} / F(L)$ & $8450(16 \%)$ & $63.5[37 \%]$ & - \\
$V_{c-} F A T$ (power) & $1.12(18 \%)$ & - & - \\
$V_{p} / F(L)$ & $15500(15 \%)$ & $80.4[36 \%]$ & - \\
Q/F (/day) & $609(28 \%)$ & $100.5[38 \%]$ & - \\
Residual errors & & - & - \\
PRO (CV\%) & $16.7(6 \%)$ & & - \\
ADD (mg/L) & $0.907(16 \%)$ & - &
\end{tabular}

FAT, fat amount; RSE, relative standard error; CV, coefficient of variation; IIV, interindividual variability; IOV, inter-occasion variability; PRO, proportional residual error; $A D D$, additive residual error; $C L / F$, apparent system clearance; $K A$, absorption rate constant; $V_{c} / F$, apparent distribution volume of central compartment; $V_{p} / F$, apparent distribution volume of peripheral compartment; $Q / F$, apparent distribution rate constant; a Every 200 days of dosing was defined as an occasion.

Fig. S6. Screen shot of the shiny app established based on the final model. a) a male patient with $85 \mathrm{~kg}$ weight and $180 \mathrm{~cm}$ height who carries G/G, A/A, and T/C for CYP2C19*2 (rs4244285), SLCO1B3 699A>G (rs7311358), and SLCO1B1 571T>C (rs4149057), respectively. b) a male patient with $85 \mathrm{~kg}$ weight and $180 \mathrm{~cm}$ height who carries G/A, A/A, and T/C for CYP2C19*2 (rs4244285), SLCO1B3 699A>G (rs7311358), and SLCO1B1 571T>C (rs4149057), respectively. c) a male patient with $85 \mathrm{~kg}$ weight and $180 \mathrm{~cm}$ height whose genotyping results are unknown. 


\section{a Mitotane}

\begin{tabular}{l} 
Characteristics of patients: \\
Is genotyping results available? \\
YES \\
CYP2C19*2 (rs4244285) \\
\hline G/G \\
SLCO1B3 669A>G (rs7311358) \\
\hline A/A \\
SLCO1B1 571T>C (rs4149057) \\
\hline T/C \\
Weight of patient (kg) \\
\hline 85 \\
Height of patient (cm) \\
\hline 180 \\
Gender of patient (F/M) \\
F \\
\hline M \\
\hline \& Plot \\
\hline
\end{tabular}

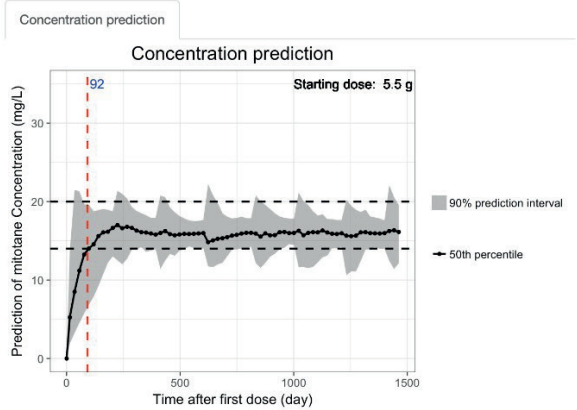

\section{b Mitotane}

\begin{tabular}{l} 
Characteristics of patients: \\
Is genotyping results available? \\
YES \\
CYP2C19*2 (rS4244285) \\
G/A \\
A/A 1 B3 669A>G (rs7311358) \\
SLCO1B1 571T>C (rs4149057) \\
\hline T/C \\
Weight of patient (kg) \\
\hline 85 \\
Height of patient (cm) \\
180 \\
Gender of patient (F/M) \\
F \\
M \\
OF Plot \\
\hline
\end{tabular}

\section{Mitotane}

\begin{tabular}{l} 
Characteristics of patients: \\
Is genotyping results available? \\
NO \\
Weight of patient (kg) \\
\hline 85 \\
Height of patient (cm) \\
\hline 180 \\
Gender of patient (F/M) \\
F \\
M \\
₹ Plot \\
\hline
\end{tabular}

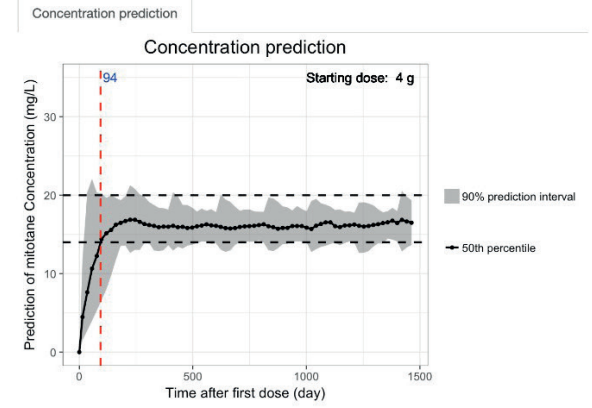

Time after first dose (day)

Concentration prediction

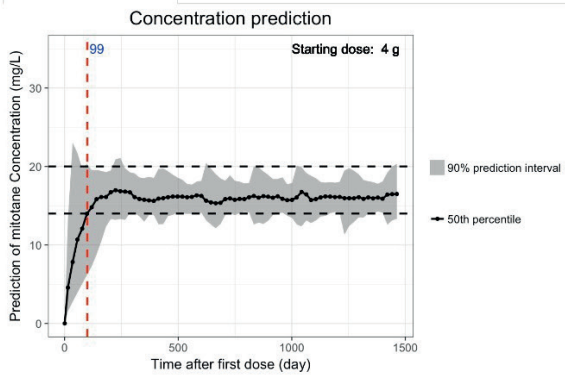


Online Resource 3 List of 959 SNPs from DMETTM array of which the genotyping results were obtained for each patient

\begin{tabular}{|c|c|c|c|c|c|}
\hline \multirow{2}{*}{$\begin{array}{l}\text { Gene } \\
\text { ABCB1 }\end{array}$} & \multicolumn{2}{|c|}{$\begin{array}{l}\text { SummaryCommon Name } \\
\text { Flag }\end{array}$} & \multirow{2}{*}{$\begin{array}{l}\text { Probe Set } \\
\text { ID } \\
\text { AM_14633 }\end{array}$} & \multirow{2}{*}{$\begin{array}{l}\text { dbSNP RS } \\
\text { ID } \\
\text { rs3213619 }\end{array}$} & \multirow{2}{*}{$\begin{array}{l}\text { Chrom } \\
\text { Ch7:87230193 }\end{array}$} \\
\hline & $N$ & ABCB1_C.-129T>C & & & \\
\hline ABCB1 & N & ABCB1_C.-1G>A & AM_14631 & rs2214102 & Ch7:87229501 \\
\hline ABCB1 & F17L & ABCB1_C.49T>C(F17L) & AM_14630 & rs28381804 & Ch7:87229452 \\
\hline ABCB1 & N21D & ABCB1_c.61A>G(N21D) & AM_14628 & rs9282564 & Ch7:87229440 \\
\hline ABCB1 & N44S & ABCB1_C.131A>G(N44S) & AM_14627 & rs1202183 & Ch7:87214983 \\
\hline ABCB1 & A80E & ABCB1_c.239C>A(A80E) & AM_14625 & rs9282565 & Ch7:87214875 \\
\hline ABCB1 & $N$ & ABCB1_c.287-25G>T & AM_14624 & rs2235015 & Ch7:87199564 \\
\hline ABCB1 & G185V & ABCB1_C.554G>T(G185V) & AM_14622 & rs1128501 & Ch7:87195534 \\
\hline ABCB1 & N & ABCB1_C.555A>T(G185G) & AM_14621 & rs1128502 & Ch7:87195533 \\
\hline ABCB1 & $N$ & ABCB1_c.729A>G(E243E) & AM_14620 & rs2235022 & Ch7:87190677 \\
\hline ABCB1 & $N$ & ABCB1_c.738G>A(A246A) & AM_14619 & rs28381867 & Ch7:87190668 \\
\hline ABCB1 & N & ABCB1_C.IVS9-44A>G & AM_14617 & rs10276036 & Ch7:87180198 \\
\hline ABCB1 & S400N & ABCB1_C.1199G>A(S400N) & AM_14616 & rs2229109 & Ch7:87179809 \\
\hline ABCB1 & $N$ & ABCB1_c.1236C>T(G412G) & AM_14612 & rs1128503 & Ch7:87179601 \\
\hline ABCB1 & $N$ & ABCB1_c.1350+44C>T & AM_14610 & rs2032588 & Ch7:87179443 \\
\hline ABCB1 & $N$ & ABCB1_C.1554+24C>T & AM_14609 & rs2235033 & Ch7:87179143 \\
\hline $\mathrm{ABCB} 1$ & $N$ & ABCB1_C.1662G>C(L554L) & AM_14607 & rs2235012 & Ch7:87178727 \\
\hline ABCB1 & E566K & ABCB1_C.1696G>A(E566K) & AM_14606 & rs28381902 & Ch7:87178693 \\
\hline ABCB1 & $N$ & ABCB1_c.1725+38A>G & AM_14605 & rs2235013 & Ch7:87178626 \\
\hline ABCB1 & R593C & ABCB1_C.1777C>T(R593C) & AM_14604 & rs28381914 & Ch7:87175289 \\
\hline ABCB1 & $N$ & ABCB1_c.1794C>T(I598I) & AM_14603 & rs28381915 & Ch7:87175272 \\
\hline ABCB1 & А599T & ABCB1_C.1795G>A(A599T) & AM_14602 & rs2235036 & Ch7:87175271 \\
\hline ABCB1 & V801M & ABCB1_c.2401G>A(V801M) & AM_14599 & rs2235039 & Ch7:87165854 \\
\hline ABCB1 & N & ABCB1_c.2481+24G>A & AM_14598 & rs2235040 & Ch7:87165750 \\
\hline ABCB1 & I829V & ABCB1_c.2485A>G(1829V) & AM_14596 & rs2032581 & Ch7:87160810 \\
\hline ABCB1 & $N$ & ABCB1_c.2505A>G(V835V) & AM_14595 & rs28381966 & Ch7:87160790 \\
\hline ABCB1 & I836V & ABCB1_c.2506A>G(1836V) & AM_14594 & rs28381967 & Ch7:87160789 \\
\hline ABCB1 & N & ABCB1_c.2650C>T(L884L) & AM_14593 & rs9282563 & Ch7:87160645 \\
\hline ABCB1 & $\begin{array}{l}\text { A893S, } \\
\text { A893T }\end{array}$ & $\begin{array}{l}\text { ABCB1_c.2677G }>T>A \\
\text { (A893SorT) }\end{array}$ & AM_14592 & rs2032582 & $\begin{array}{l}\text { Ch7:87160618 } \\
\text { Ch7:87160618 }\end{array}$ \\
\hline ABCB1 & A999T & ABCB1_c.2995G>A(A999T) & AM_14591 & rs72552784 & Ch7:87145914 \\
\hline ABCB1 & $\mathrm{N}$ & ABCB1_C.3084G>A(P1028P) & AM_14590 & rs2235044 & Ch7:87145825 \\
\hline ABCB1 & P1051A & ABCB1_c.3151C>G(P1051A) & AM_14589 & rs28401798 & Ch7:87144678 \\
\hline ABCB1 & G1063A & ABCB1_c.3188G>C(G1063A) & AM_14588 & rs2707944 & Ch7:87144641 \\
\hline ABCB1 & N & ABCB1_c.3189C>G(G1063G) & AM_14587 & rs2707943 & Ch7:87144640 \\
\hline ABCB1 & S1141T & ABCB1_c.3421T>A(S1141T) & AM_14582 & rs2229107 & Ch7:87138659 \\
\hline ABCB1 & N & ABCB1_C.3435C>T(|1145I) & AM_14581 & rs1045642 & Ch7:87138645 \\
\hline ABCB1 & N & ABCB1_c.3747C>G(G1249G) & AM_14579 & rs2235051 & Ch7:87133655 \\
\hline ABCB1 & V1251I & ABCB1_C.3751G>A(V1251I) & AM_14578 & rs28364274 & Ch7:87133651 \\
\hline ABCB1 & N & ABCB1_c.*89A>T(3'UTR) & AM_14577 & rs17064 & Ch7:87133470 \\
\hline ABCB1 & N & ABCB1_C.*193A>G(3'UTR) & AM_14575 & rs3842 & Ch7:87133366 \\
\hline ABCB4 & N & ABCB4_C.-1921T>C & AM_14572 & rs3747806 & Ch7:87106702 \\
\hline
\end{tabular}




\begin{tabular}{|c|c|c|c|c|c|}
\hline \multirow{2}{*}{$\begin{array}{l}\text { Gene } \\
A B C B 4\end{array}$} & \multicolumn{2}{|c|}{$\begin{array}{l}\text { SummaryCommon Name } \\
\text { Flag }\end{array}$} & \multirow{2}{*}{$\begin{array}{l}\text { Probe Set } \\
\text { ID } \\
\text { AM_14570 }\end{array}$} & \multirow{2}{*}{$\begin{array}{l}\text { dbSNP RS } \\
\text { ID } \\
\text { rs4148805 }\end{array}$} & \multirow{2}{*}{$\begin{array}{l}\text { Chrom } \\
\text { Ch7:87106365 }\end{array}$} \\
\hline & $\mathrm{N}$ & ABCB4_C.-1584C>T & & & \\
\hline ABCB4 & N & ABCB4_C.-1484T >C & AM_14569 & rs4148806 & Ch7:87106265 \\
\hline ABCB4 & $\mathrm{N}$ & ABCB4_C.-1031C>T & AM_14568 & rs4148807 & Ch7:87105812 \\
\hline ABCB4 & $\mathrm{N}$ & ABCB4_C.-1014A>G & AM_14567 & rs4148808 & Ch7:87105795 \\
\hline ABCB4 & $\mathrm{N}$ & ABCB4_c.147C>T(S49S) & AM_14563 & rs8187789 & Ch7:87092213 \\
\hline ABCB4 & N & ABCB4_C.175C>T(L59L) & AM_14562 & rs2302387 & Ch7:87092185 \\
\hline ABCB4 & L73V & ABCB4_c.217C>G(L73V) & AM_14561 & rs8187788 & Ch7:87092143 \\
\hline ABCB4 & $\mathrm{N}$ & ABCB4_C.240G>A(E80E) & AM_14560 & rs8187787 & Ch7:87092120 \\
\hline ABCB4 & R144X & ABCB4_C.430C $>T(R 144 X)$ & AM_14558 & rs72552780 & Ch7:87082366 \\
\hline ABCB4 & N & ABCB4_C.504C>T(N168N) & AM_14556 & rs1202283 & Ch7:87082292 \\
\hline ABCB4 & N & ABCB4_c.696C >T(A232A) & AM_14554 & rs8187791 & Ch7:87080951 \\
\hline ABCB4 & N & ABCB4_C.711A>T(|237|) & AM_14553 & rs2109505 & Ch7:87079406 \\
\hline ABCB4 & L238V & ABCB4_c.712C>G(L238V) & AM_14552 & rs45596335 & Ch7:87079405 \\
\hline ABCB4 & E528D & ABCB4_C.1584G>C(E528D) & AM_14546 & rs8187797 & Ch7:87069130 \\
\hline ABCB4 & Q636X & ABCB4_C.1906C>T(Q636X) & AM_14542 & rs72552775 & Ch7:87056224 \\
\hline ABCB4 & R652G & ABCB4_c.1954A>G(R652G) & AM_14540 & rs2230028 & Ch7:87056176 \\
\hline ABCB4 & W658X & ABCB4_c.1973G>A(W658X) & AM_14539 & rs72552774 & Ch7:87056157 \\
\hline ABCB4 & N & ABCB4_C.2325G >C(T775T) & AM_14537 & rs8187802 & Ch7:87049383 \\
\hline ABCB4 & R788Q & ABCB4_c.2363G>A(R788Q) & AM_14536 & rs8187801 & Ch7:87049345 \\
\hline ABCB4 & N & ABCB4_c.2559T>G(G853G) & AM_14535 & rs3761810 & Ch7:87046751 \\
\hline ABCB4 & N & ABCB4_c.2952A>G(A984A) & AM_14533 & rs45574932 & Ch7:87038681 \\
\hline ABCB4 & $\mathrm{N}$ & ABCB4_C.3111T>C(N1037N) & AM_14532 & rs8187808 & Ch7:87037521 \\
\hline ABCB4 & N & ABCB4_c.3144C $>$ T(N1048N) & AM_14531 & rs8187807 & Ch7:87037488 \\
\hline ABCB4 & A1100T & ABCB4_c.3298G>A(A1100T) & AM_14530 & rs31655 & Ch7:87035792 \\
\hline ABCB4 & I1185V & ABCB4_c.3553A>G(11185V) & AM_14529 & rs8187811 & Ch7:87032531 \\
\hline ABCB4 & N & ABCB4_c.*509T>C & AM_14528 & rs2097937 & Ch7:87030903 \\
\hline ABCB7 & N & ABCB7_c.457-2518A>G & AM_15425 & rs5937939 & ChX:74299007 \\
\hline ABCB7 & N & ABCB7_c.457-1932A>T & AM_15424 & rs4892538 & ChX:74298421 \\
\hline ABCB7 & R259K & ABCB7_c.776G>A(R259K) & AM_15423 & rs1054913 & ChX:74295279 \\
\hline ABCB7 & L272P & ABCB7_C.815T>C(L272P) & AM_15422 & rs1054914 & ChX:74295240 \\
\hline ABCB7 & A581V & ABCB7_c.1742C>T(A581V) & AM_15415 & rs1340989 & ChX:74284997 \\
\hline $\mathrm{ABCB} 7$ & N & ABCB7_c.1743A>G(A581A) & AM_15414 & rs1340990 & ChX:74284996 \\
\hline ABCB11 & N & ABCB11_C.-12519C>T & AM_12785 & rs4148768 & Ch2:169887154 \\
\hline ABCB11 & N & ABCB11_C.-10013G>A & AM_12783 & rs4668115 & Ch2:169884648 \\
\hline ABCB11 & N & ABCB11_c.-8583G>A & AM_12782 & rs3770603 & Ch2:169883218 \\
\hline ABCB11 & N & ABCB11_C.-7449T>C & AM_12781 & rs4148770 & Ch2:169882084 \\
\hline ABCB11 & N & ABCB11_C.-7056C>T & AM_12780 & rs3770602 & Ch2:169881691 \\
\hline ABCB11 & N & ABCB11_C.-5543C>T & AM_12778 & rs7602171 & Ch2:169880178 \\
\hline ABCB11 & N & ABCB11_C.-2450A>G & AM_12776 & rs7578587 & Ch2:169877085 \\
\hline ABCB11 & N & ABCB11_C.-904C>A & AM_12775 & rs3755163 & Ch2:169875539 \\
\hline ABCB11 & N & ABCB11_c.-899delCT & AM_12772 & rs4148771 & Ch2:169875534 \\
\hline ABCB11 & N & ABCB11_c.-899delCT_alternate & AM_12773 & rs4148771 & $\begin{array}{l}\text { Ch2:169875534 } \\
\text { Ch2:169875534 }\end{array}$ \\
\hline ABCB11 & N & ABCB11_C.-610C>A & AM_12771 & rs3755162 & Ch2:169875245 \\
\hline ABCB11 & N & ABCB11_C.99-18T>C & AM_12767 & rs4148776 & Ch2:169870882 \\
\hline
\end{tabular}




\begin{tabular}{|c|c|c|c|c|c|}
\hline \multirow{2}{*}{$\begin{array}{l}\text { Gene } \\
\text { ABCB11 }\end{array}$} & \multicolumn{2}{|c|}{$\begin{array}{l}\text { SummaryCommon Name } \\
\text { Flag }\end{array}$} & \multirow{2}{*}{$\begin{array}{l}\text { Probe Set } \\
\text { ID } \\
\text { AM_12766 }\end{array}$} & \multirow{2}{*}{$\begin{array}{l}\text { dbSNP RS } \\
\text { ID } \\
\text { rs3815675 }\end{array}$} & \multirow{2}{*}{$\begin{array}{l}\text { Chrom } \\
\text { Ch2:169870855 }\end{array}$} \\
\hline & $\mathrm{N}$ & ABCB11_C.108T>C(D36D) & & & \\
\hline ABCB11 & $\mathrm{N}$ & ABCB11_c.270T>C(F90F) & AM_12762 & rs4148777 & Ch2:169869901 \\
\hline ABCB11 & G238V & ABCB11_c.713G>T(G238V) & AM_12749 & rs72551306 & Ch2:169850291 \\
\hline ABCB11 & N & ABCB11_C.807T>C(Y269Y) & AM_12744 & rs2287616 & Ch2:169847412 \\
\hline ABCB11 & R299K & ABCB11_C.896G>A(R299K) & AM_12742 & rs2287617 & Ch2:169847323 \\
\hline ABCB11 & N & ABCB11_C.957A>G(G319G) & AM_12739 & rs7563233 & Ch2:169842746 \\
\hline ABCB11 & A444V & ABCB11_c.1331C>T(A444V) & AM_12726 & rs2287622 & Ch2:169830328 \\
\hline ABCB11 & D482G & ABCB11_c.1445A>G(D482G) & AM_12722 & rs72549402 & Ch2:169828550 \\
\hline ABCB11 & $\mathrm{R} 575 \mathrm{X}$ & ABCB11_c.1723C $>$ T(R575X) & AM_12719 & rs72549401 & Ch2:169826641 \\
\hline ABCB11 & N & ABCB11_c.2412A>G(A804A) & AM_12688 & rs11568373 & Ch2:169801403 \\
\hline ABCB11 & G982R & ABCB11_c.2944G>A(G982R) & AM_12677 & rs72549399 & Ch2:169791806 \\
\hline ABCB11 & N & ABCB11_C.3084A>G(A1028A) & AM_12674 & rs497692 & Ch2:169789016 \\
\hline ABCB11 & R1057X & ABCB11_c.3169C>T(R1057X) & AM_12672 & rs72549397 & Ch2:169788931 \\
\hline ABCB11 & R1090X & ABCB11_c.3268C>T(R1090X) & AM_12670 & rs72549396 & Ch2:169787318 \\
\hline ABCB11 & R1153C & ABCB11_C.3457C>T(R1153C) & AM_12667 & rs72549395 & Ch2:169783827 \\
\hline ABCB11 & E1186K & ABCB11_C.3556G>A(E1186K) & AM_12666 & rs1521808 & Ch2:169783728 \\
\hline ABCB11 & R1268Q & ABCB11_c.3803G>A(R1268Q) & AM_12660 & rs72549394 & Ch2:169780295 \\
\hline ABCB11 & N & ABCB11_C.*236G>A & AM_12658 & rs473351 & Ch2:169779896 \\
\hline ABCB11 & N & ABCB11_c.*281T>G & AM_12657 & rs3732038 & Ch2:169779851 \\
\hline ABCB11 & N & ABCB11_C.*368G>A & AM_12656 & rs495714 & Ch2:169779764 \\
\hline ABCB11 & N & ABCB11_c.*420A>G & AM_12655 & rs496550 & Ch2:169779712 \\
\hline $\mathrm{ABCC} 1$ & S92F & ABCC1_c.275C >T(S92F) & AM_10915 & rs8187844 & Ch16:16103682 \\
\hline $\mathrm{ABCC} 1$ & R230Q & ABCC1_c.689G>A(R230Q) & AM_10917 & rs8187848 & Ch16:16130340 \\
\hline $\mathrm{ABCC} 1$ & N & ABCC1_c.825T>C(V275V) & AM_10920 & rs246221 & Ch16:16138322 \\
\hline $\mathrm{ABCC} 1$ & V353M & ABCC1_c.1057G>A(V353M) & AM_10922 & rs8187852 & Ch16:16139709 \\
\hline $\mathrm{ABCC} 1$ & $\mathrm{~N}$ & ABCC1_C.1068G>A(T356T) & AM_10924 & rs8187853 & Ch16:16139720 \\
\hline $\mathrm{ABCC} 1$ & R433S & ABCC1_C.1299G $>$ T(R433S) & AM_10925 & rs60782127 & Ch16:16142079 \\
\hline $\mathrm{ABCC} 1$ & $\begin{array}{l}\text { D526A, } \\
526 F S\end{array}$ & $\begin{array}{l}\text { ABCC1_C.1577A>CorCA } \\
\text { (D526AorX) }\end{array}$ & AM_10926 & rs72547522 & $\begin{array}{l}\text { Ch16:16150052, } \\
\text { Ch16:16150052 }\end{array}$ \\
\hline $\mathrm{ABCC} 1$ & N & ABCC1_c.1704C>T(Y568Y) & AM_10928 & rs8187858 & Ch16:16162039 \\
\hline $\mathrm{ABCC} 1$ & N & ABCC1_c.1911C>T(D637D) & AM_10929 & rs8187859 & Ch16:16165585 \\
\hline $\mathrm{ABCC} 1$ & N & ABCC1_c.2001C>T(S667S) & AM_10930 & rs8187863 & Ch16:16173221 \\
\hline $\mathrm{ABCC} 1$ & N & ABCC1_C.2007C>T(P669P) & AM_10931 & rs2301666 & Ch16:16173227 \\
\hline $\mathrm{ABCC} 1$ & R723Q & ABCC1_c.2168G>A(R723Q) & AM_10932 & rs4148356 & Ch16:16177275 \\
\hline $\mathrm{ABCC} 1$ & N & ABCC1_C.3282G>A(P1094P) & AM_10936 & rs4148377 & Ch16:16215891 \\
\hline $\mathrm{ABCC} 1$ & $\mathrm{~T} 1345 \mathrm{M}$ & ABCC1_C.4034C>T(T1345M) & AM_10939 & rs8057331 & Ch16:16230411 \\
\hline $\mathrm{ABCC} 1$ & N & ABCC1_c.*866T>A & AM_10944 & rs212090 & Ch16:16236004 \\
\hline $\mathrm{ABCC} 1$ & N & ABCC1_C.*1293G>A & AM_10945 & rs4148380 & Ch16:16236431 \\
\hline $\mathrm{ABCC} 1$ & $\mathrm{~N}$ & ABCC1_C.*1385G>T & AM_10946 & rs8056298 & Ch16:16236523 \\
\hline $\mathrm{ABCC} 1$ & N & ABCC1_C.*1512T>C & AM_10947 & rs212091 & Ch16:16236650 \\
\hline $\mathrm{ABCC2}$ & N & ABCC2_C.-24C>T(5'UTR) & AM_10143 & rs717620 & Ch10:101542578 \\
\hline $\mathrm{ABCC} 2$ & N & ABCC2_C.-23G>A(5'UTR) & AM_10144 & rs17216156 & Ch10:101542579 \\
\hline $\mathrm{ABCC2}$ & F39Y & ABCC2_C.116T $>A(F 39 Y)$ & AM_10145 & rs927344 & Ch10:101544447 \\
\hline ABCC2 & N & ABCC2_c.159A>G(K53K) & AM_10146 & rs17222596 & Ch10:101544490 \\
\hline $\mathrm{ABCC} 2$ & M246L & ABCC2_c.736A>C(M246L) & AM_10147 & rs17222744 & Ch10:101556957 \\
\hline
\end{tabular}




\begin{tabular}{|c|c|c|c|c|c|}
\hline \multirow{2}{*}{$\begin{array}{l}\text { Gene } \\
\mathrm{ABCC} 2\end{array}$} & \multicolumn{2}{|c|}{$\begin{array}{l}\text { SummaryCommon Name } \\
\text { Flag }\end{array}$} & \multirow{2}{*}{$\begin{array}{l}\text { Probe Set } \\
\text { ID } \\
\text { AM_10149 }\end{array}$} & \multirow{2}{*}{$\begin{array}{l}\text { dbSNP RS } \\
\text { ID } \\
\text { rs17222674 }\end{array}$} & \multirow{2}{*}{$\begin{array}{l}\text { Chrom } \\
\text { Ch10:101559094 }\end{array}$} \\
\hline & D333G & ABCC2_c.998A>G(D333G) & & & \\
\hline $\mathrm{ABCC} 2$ & R353H & ABCC2_C.1058G>A(R353H) & AM_10150 & rs7080681 & Ch10:101560169 \\
\hline ABCC2 & $\mathrm{N}$ & ABCC2_c.1219C>T(L407L) & AM_10151 & rs17216198 & Ch10:101563785 \\
\hline $\mathrm{ABCC} 2$ & V417l & ABCC2_c.1249G>A(V417I) & AM_10152 & rs2273697 & Ch10:101563815 \\
\hline $\mathrm{ABCC2}$ & N & ABCC2_c.1434G>T>A(A478AorA) & AM_10153 & rs4267009 & $\begin{array}{l}\text { Ch10:101564000, } \\
\text { Ch10:101564000 }\end{array}$ \\
\hline $\mathrm{ABCC} 2$ & T4861 & ABCC2_c.1457C>T(T486I) & AM_10154 & rs17222589 & Ch10:101564023 \\
\hline ABCC2 & K495E & ABCC2_c.1483A>G(K495E) & AM_10155 & rs17222561 & Ch10:101565157 \\
\hline ABCC2 & F562L & ABCC2_c.1686T>G(F562L) & AM_10156 & rs17216233 & Ch10:101567857 \\
\hline ABCC2 & $602 \mathrm{FS}$ & ABCC2_c.1803insC & AM_10157 & rs72558198 & Ch10:101567974 \\
\hline $\mathrm{ABCC} 2$ & 1670T & ABCC2_c.2009T>C(1670T) & AM_10158 & rs17222632 & Ch10:101572816 \\
\hline ABCC2 & $\mathrm{N}$ & ABCC2_c.2073C>A(V691V) & AM_10159 & rs17222624 & Ch10:101572880 \\
\hline ABCC2 & N718S & ABCC2_C.2153A>G(N718S) & AM_10160 & rs3740072 & Ch10:101577123 \\
\hline ABCC2 & R768W & ABCC2_C.2302C>T(R768W) & AM_10161 & rs56199535 & Ch10:101578577 \\
\hline $\mathrm{ABCC} 2$ & S789F & ABCC2_c.2366C>T(S789F) & AM_10162 & rs56220353 & Ch10:101578641 \\
\hline $\mathrm{ABCC} 2$ & L849R & ABCC2_c.2546T>G(L849R) & AM_10163 & rs17222617 & 78952 \\
\hline $\mathrm{ABCC} 2$ & E893Q & ABCC2_c.2677G>C(E893Q) & AM_10 & rs3740 & 90120 \\
\hline $\mathrm{ABCC2}$ & Y967X & ABCC2_c.2901C>A(Y967X) & AM_10166 & rs17222547 & Ch10:101591385 \\
\hline $\mathrm{ABCC} 2$ & $\mathrm{~N}$ & ABCC2_c.2934G>A(S978S) & AM_10167 & rs3740070 & Ch10:101591418 \\
\hline $\mathrm{ABCC} 2$ & $1982 \mathrm{~V}$ & ABCC2_c.2944A>G(1982V) & AM_10168 & rs17222554 & Ch10:101591428 \\
\hline ABCC2 & 11036T & ABCC2_C.3107T>C(11036T) & AM_10169 & rs45441199 & Ch10:101591737 \\
\hline ABCC2 & R1066X & ABCC2_c.3196C $>$ T(R1066X) & AM_10171 & rs72558199 & Ch10:101591826 \\
\hline ABCC2 & $\mathrm{N}$ & ABCC2_c.3396T>C(I1132I) & AM_10172 & rs17216345 & Ch10:101594274 \\
\hline ABCC2 & 11173F & ABCC2_C.3517A>T(I1173F) & AM_10174 & rs72558201 & Ch10:101595950 \\
\hline ABCC2 & R1181L & ABCC2_c.3542G>T(R1181L) & $\mathrm{AM}_{-} 1$ & rs8187692 & Ch10:101595975 \\
\hline ABCC2 & $\mathrm{N}$ & ABCC2_C.3561G>A(E1187E) & AM_10176 & rs17216324 & Ch10:101595994 \\
\hline ABCC2 & V1188E & ABCC2_c.3563T>A(V1188E) & AM_10177 & rs17222723 & Ch10:101595996 \\
\hline $\mathrm{ABCC} 2$ & T1273A & ABCC2_C.3817A>G(T1273A) & AM_10178 & rs8187699 & Ch10:101603631 \\
\hline $\mathrm{ABCC} 2$ & P1291L & ABCC2_C.3872C>T(P1291L) & AM_10179 & rs17216317 & Ch10:101604107 \\
\hline $\mathrm{ABCC} 2$ & K1299Q & ABCC2_C.3895A>C(K1299Q) & AM_10180 & rs4148400 & Ch10:101604130 \\
\hline ABCC2 & $\mathrm{N}$ & ABCC2_C.3927C>T(Y1309Y) & AM_10181 & rs4148401 & Ch10:101604162 \\
\hline $\mathrm{ABCC} 2$ & R1310X & ABCC2_C.3928C>T(R1310X) & AM_10182 & rs66898362 & Ch10:101604163 \\
\hline $\mathrm{ABCC2}$ & N & ABCC2_c.3972C>T(|1324I) & AM_10183 & rs3740066 & Ch10:101604207 \\
\hline $\mathrm{ABCC} 2$ & N & ABCC2_C.4062C>T(A1354A) & AM_10184 & rs17216275 & Ch10:101605455 \\
\hline ABCC2 & $\mathrm{N}$ & ABCC2_C.4110C>T(L1370L) & AM_10185 & rs7899457 & Ch10:101605503 \\
\hline ABCC2 & $\mathrm{N}$ & ABCC2_c.4290G $>T(V 1430 V)$ & AM_10188 & rs1137968 & Ch10:101606861 \\
\hline $\mathrm{ABCC2}$ & $\begin{array}{l}\text { A1450S, } \\
\text { A1450T }\end{array}$ & ABCC2_c.4348G>T>A(A1450SorT) & AM_10189 & rs56296335 & $\begin{array}{l}\text { Ch10:101610393, } \\
\text { Ch10:101610393 }\end{array}$ \\
\hline $\mathrm{ABCC} 2$ & N & ABCC2_C.4410G>A(E1470E) & AM_10190 & rs8187706 & Ch10:101610455 \\
\hline ABCC2 & N & ABCC2_C.4488C>T(H1496H) & AM_10191 & rs8187707 & Ch10:101610533 \\
\hline $\mathrm{ABCC} 2$ & N & ABCC2_c.4527C>T(N1509N) & AM_10192 & rs8187709 & Ch10:101611277 \\
\hline ABCC2 & C1515Y & ABCC2_C.4544G>A(C1515Y) & AM_10193 & rs8187710 & Ch10:101611294 \\
\hline ABCC3 & A528G & ABCC3_c.1583C>G(A528G) & AM_11225 & rs1003355 & Ch17:48745066 \\
\hline $\mathrm{ABCC} 3$ & N & ABCC3_c.3039C>T(G1013G) & AM_11231 & rs4148416 & Ch17:48753423 \\
\hline ABCC3 & R1297H & ABCC3_c.3890G $>A(R 1297 H)$ & AM_11235 & rs11568591 & Ch17:48761053 \\
\hline
\end{tabular}




\begin{tabular}{|c|c|c|c|c|c|}
\hline \multirow{2}{*}{$\begin{array}{l}\text { Gene } \\
\mathrm{ABCC} 3\end{array}$} & \multicolumn{2}{|c|}{$\begin{array}{l}\text { SummaryCommon Name } \\
\text { Flag }\end{array}$} & \multirow{2}{*}{$\begin{array}{l}\text { Probe Set } \\
\text { ID } \\
\text { AM_11236 }\end{array}$} & \multirow{2}{*}{$\begin{array}{l}\text { dbSNP RS } \\
\text { ID } \\
\text { rs } 2277624\end{array}$} & \multirow{2}{*}{$\begin{array}{l}\text { Chrom } \\
\text { Ch17:48761105 }\end{array}$} \\
\hline & $\mathrm{N}$ & ABCC3_c.3942C $>T(H 1314 H)$ & & & \\
\hline $\mathrm{ABCC} 3$ & L1362V & ABCC3_c.4084C>G(L1362V) & AM_11239 & rs1051625 & Ch17:48761439 \\
\hline $\mathrm{ABCC} 3$ & $N$ & ABCC3_c.4509A>G(E1503E) & AM_11243 & rs1051640 & Ch17:48768486 \\
\hline $\mathrm{ABCC} 4$ & C171G & ABCC4_C.511T>G(C171G) & AM_10640 & rs4148460 & Ch13:95886884 \\
\hline $\mathrm{ABCC} 4$ & K304N & ABCC4_c.912G>T(K304N) & AM_10634 & rs2274407 & Ch13:95859035 \\
\hline $\mathrm{ABCC} 4$ & N & ABCC4_C.951A>G(R317R) & AM_10633 & rs2274406 & Ch13:95858996 \\
\hline $\mathrm{ABCC} 4$ & N & ABCC4_c.969G>A(S323S) & AM_10632 & rs2274405 & Ch13:95858978 \\
\hline $\mathrm{ABCC} 4$ & $631 \mathrm{FS}$ & ABCC4_C.1892delT(L631X) & AM_10624 & rs72559753 & Ch13:95818554 \\
\hline $\mathrm{ABCC} 4$ & E757K & ABCC4_C.2269G>A(E757K) & AM_10622 & rs3765534 & Ch13:95815415 \\
\hline $\mathrm{ABCC} 4$ & N & ABCC4_C.2712G>A(L904L) & AM_10618 & rs1678339 & Ch13:95727780 \\
\hline $\mathrm{ABCC} 4$ & N & ABCC4_C.2844C>T(F948F) & AM_10617 & rs1189466 & Ch13:95726541 \\
\hline $\mathrm{ABCC} 4$ & $\mathrm{~N}, 1116 \mathrm{FS}$ & $\begin{array}{l}\text { ABCC4_c.3348A>GorDel } \\
\text { (K1116KorX) }\end{array}$ & AM_10611 & rs1751034 & $\begin{array}{l}\text { Ch13:95714976, } \\
\text { Ch13:95714976 }\end{array}$ \\
\hline $\mathrm{ABCC} 4$ & N & ABCC4_C.*38T >G & AM_10604 & rs3742106 & Ch13:95673791 \\
\hline $\mathrm{ABCC} 4$ & N & ABCC4_C.*311G>A & AM_10603 & rs4148551 & Ch13:95673518 \\
\hline $\mathrm{ABCC} 4$ & N & ABCC4_C.*694G>A & AM_10602 & rs4148553 & Ch13:95673135 \\
\hline $\mathrm{ABCC} 4$ & N & ABCC4_C.*879T>C & AM_10601 & rs1059751 & Ch13:95672950 \\
\hline $\mathrm{ABCC} 4$ & N & ABCC4_C.*1282T>C & AM_10600 & rs4148554 & Ch13:95672547 \\
\hline $\mathrm{ABCC} 4$ & N & ABCC4_C.*1351T>C & AM_10599 & rs1059754 & Ch13:95672478 \\
\hline $\mathrm{ABCC} 4$ & $N$ & ABCC4_C.*1564A>T & AM_10598 & rs4148555 & Ch13:95672265 \\
\hline ABCC5 & $N$ & ABCC5_c.1146A>G(Q382Q) & AM_13400 & rs7636910 & Ch3:183699516 \\
\hline ABCC5 & $N$ & ABCC5_C.1200C>T(S400S) & AM_13397 & rs1053386 & Ch3:183696387 \\
\hline $\mathrm{ABCC} 5$ & N & ABCC5_c.1782C>T(C594C) & AM_13393 & rs939336 & Ch3:183685534 \\
\hline $\mathrm{ABCC5}$ & 1147FS & ABCC5_c.3441_3442insC(V1147X) & AM_13381 & rs72551384 & Ch3:183663700 \\
\hline $\mathrm{ABCC} 5$ & Y1202X & ABCC5_c.3606C >A(Y1202X) & AM_13378 & rs1053351 & Ch3:183660603 \\
\hline ABCC5 & N & ABCC5_c.3624C>T(L1208L) & AM_13377 & rs3749442 & Ch3:183660585 \\
\hline $\mathrm{ABCC5}$ & T1383N & ABCC5_C.4148C $>A(T 1383 N)$ & AM_13370 & rs1053387 & Ch3:183643407 \\
\hline $\mathrm{ABCC5}$ & N & ABCC5_C.*1243G>A & AM_13365 & rs562 & Ch3:183637845 \\
\hline $\mathrm{ABCC5}$ & N & ABCC5_C.*1366A>C & AM_13364 & rs3805114 & Ch3:183637722 \\
\hline ABCC6 & N & ABCC6_C.1890C>G(T630T) & AM_10978 & rs8058696 & Ch16:16278869 \\
\hline ABCC6 & H632Q & ABCC6_c.1896C>A(H632Q) & AM_10977 & rs8058694 & Ch16:16278863 \\
\hline ABCC6 & V665A & ABCC6_C.1994T>C(V665A) & AM_10976 & rs4341770 & Ch16:16276737 \\
\hline ABCC6 & N & ABCC6_c.2400G>A(G800G) & AM_10968 & rs7500834 & Ch16:16272670 \\
\hline ABCC6 & V848M & ABCC6_c.2542G>A(V848M) & AM_10966 & rs6416668 & Ch16:16271357 \\
\hline ABCC6 & N & ABCC6_c.2835C>T(P945P) & AM_10964 & rs2856585 & Ch16:16263663 \\
\hline ABCC6 & R1268Q & ABCC6_C.3803G>A(R1268Q) & AM_10959 & rs2238472 & Ch16:16251599 \\
\hline $\mathrm{ABCC} 8$ & $\mathrm{R} 248 \mathrm{X}$ & ABCC8_C.742C $>T(R 248 X)$ & AM_10301 & rs72559730 & Ch11:17483210 \\
\hline $\mathrm{ABCC} 8$ & $631 \mathrm{FS}$ & ABCC8_C.1891delC(P631X) & AM_10287 & rs72559725 & Ch11:17450144 \\
\hline $\mathrm{ABCC} 8$ & $701 \mathrm{FS}$ & ABCC8_c.2101_2102insT(I701X) & AM_10285 & rs72559724 & Ch11:17449428 \\
\hline $\mathrm{ABCC} 8$ & R836X & ABCC8_c.2506C>T(R836X) & AM_10281 & rs72559722 & Ch11:17434263 \\
\hline $\mathrm{ABCC} 8$ & L1014X & ABCC8_c.3041T>A(L1014X) & AM_10277 & rs67706538 & Ch11:17428556 \\
\hline $\mathrm{ABCC} 8$ & S1369A & ABCC8_c.4105T>G(S1369A) & AM_10270 & rs757110 & Ch11:17418477 \\
\hline $\mathrm{ABCC} 8$ & V1572I & ABCC8_c.4714G >A(V1572I) & AM_10260 & rs8192690 & Ch11:17414570 \\
\hline ABCC9 & N & ABCC9_c.669G>T(L223L) & AM_10558 & rs17846788 & Ch12:22068749 \\
\hline ABCC9 & N & ABCC9_c.789C>T(C263C) & AM_10557 & rs58386780 & Ch12:22068629 \\
\hline
\end{tabular}




\begin{tabular}{|c|c|c|c|c|c|}
\hline \multirow{2}{*}{$\begin{array}{l}\text { Gene } \\
\text { ABCC9 }\end{array}$} & \multicolumn{2}{|c|}{$\begin{array}{l}\text { SummaryCommon Name } \\
\text { Flag }\end{array}$} & \multirow{2}{*}{$\begin{array}{l}\text { Probe Set } \\
\text { ID } \\
\text { AM_10556 }\end{array}$} & \multirow{2}{*}{$\begin{array}{l}\text { dbSNP RS } \\
\text { ID } \\
\text { rs72559752 }\end{array}$} & \multirow{2}{*}{$\begin{array}{l}\text { Chrom } \\
\text { Ch12:22063889 }\end{array}$} \\
\hline & $\mathrm{N}$ & ABCC9_c.1035G>A(K345K) & & & \\
\hline ABCC9 & $\mathrm{N}$ & ABCC9_C.1296C $>$ T(P432P) & AM_10555 & rs10770865 & Ch12:22063115 \\
\hline ABCC9 & $\mathrm{N}$ & ABCC9_c.1848C>T(D616D) & AM_10554 & rs61001398 & Ch12:22040823 \\
\hline ABCC9 & A1513T & ABCC9_c.4537G>A(A1513T) & AM_10546 & rs72559751 & Ch12:21958221 \\
\hline ABCG1 & $\mathrm{N}$ & ABCG1_C.589-407A>G & AM_12204 & rs492338 & Ch21:43701977 \\
\hline ABCG1 & N & ABCG1_c.973+672G>A & AM_12205 & rs3788007 & Ch21:43706776 \\
\hline$A B C G 1$ & $\mathrm{~N}$ & ABCG1_C.974-898C>G & AM_12206 & rs425215 & Ch21:43707101 \\
\hline $\mathrm{ABCG} 1$ & $\mathrm{~N}$ & ABCG1_c.1430-293C>G & AM_12207 & rs914189 & Ch21:43710909 \\
\hline ABCG1 & $\mathrm{N}$ & ABCG1_c.1809-252G>A & AM_12208 & rs3788010 & Ch21:43716022 \\
\hline ABCG1 & $\mathrm{N}$ & ABCG1_c.*399G>A & AM_12209 & rs1044317 & Ch21:43716901 \\
\hline ABCG1 & $\mathrm{N}$ & ABCG1_C.*1981G>A & AM_12210 & rs1541290 & Ch21:43718483 \\
\hline ABCG2 & $\mathrm{N}$ & ABCG2_C.369C>T(Y123Y) & AM_13690 & rs2231139 & Ch4:89052964 \\
\hline ABCG2 & Q126X & ABCG2_c.376C>T(Q126X) & AM_13689 & rs72552713 & Ch4:89052957 \\
\hline ABCG2 & Q141K & ABCG2_c.421C>A(Q141K) & AM_13688 & rs2231142 & Ch4:89052323 \\
\hline ABCG2 & Q166E & ABCG2_c.496C>G(Q166E) & AM_13687 & rs1061017 & Ch4:89052248 \\
\hline ABCG2 & $N$ & ABCG2_C.564A>G(G188G) & AM_13686 & rs3116439 & Ch4:89042912 \\
\hline ABCG2 & F208S & ABCG2_c.623T>C(F208S) & AM_13683 & rs1061018 & Ch4:89042853 \\
\hline ABCG2 & $\mathrm{S} 248 \mathrm{P}$ & ABCG2_C.742T>C(S248P) & AM_13682 & rs3116448 & Ch4:89039360 \\
\hline ABCG2 & E334X & ABCG2_C.1000G>T(E334X) & AM_13681 & rs3201997 & Ch4:89034649 \\
\hline ATP7A & L142V & ATP7A_c.424C>G(L142V) & AM_15437 & rs61743418 & ChX:77244041 \\
\hline ATP7A & Q167X & ATP7A_c.499C>T(Q167X) & AM_15438 & rs72554635 & ChX:77244116 \\
\hline ATP7A & I189V & ATP7A_c.565A>G(1189V) & AM_15439 & rs2228447 & ChX:77244182 \\
\hline ATP7A & R409X & ATP7A_C.1225C>T(R409X) & AM_15440 & rs72554636 & ChX:77245343 \\
\hline ATP7A & $\mathrm{N}$ & ATP7A_C.1390T>C(L464L) & AM_15441 & rs2234934 & ChX:77254028 \\
\hline ATP7A & V767L & ATP7A_c.2299G>C(V767L) & AM_15454 & rs2227291 & ChX:77268502 \\
\hline ATP7A & V1401L & ATP7A_C.4201G>C(V1401L) & AM_15472 & rs5959130 & ChX:77301044 \\
\hline CDA & *2 & $C D A * 2$ C.79A>C(K27Q) & AM_11499 & rs2072671 & Ch1:20915701 \\
\hline CDA & *3 & CDA*3_c.208G>A(A70T) & AM_11506 & rs60369023 & Ch1:20931474 \\
\hline CDA & $\mathrm{N}$ & CDA_C.-88G $>A$ & AM_11498 & rs602946 & Ch1:20915535 \\
\hline CDA & $\mathrm{N}$ & CDA_C.154+1015A>G & AM_11500 & rs818202 & Ch1:20916791 \\
\hline CDA & $\mathrm{N}$ & CDA_C.154+3136T>C & AM_11501 & rs10916824 & Ch1:20918912 \\
\hline CDA & $\mathrm{N}$ & CDA_c.435C>T(T145T) & AM_11520 & rs1048977 & Ch1:20945055 \\
\hline CES2 & N & CES2_c.268+947A>T & AM_11065 & rs11568314 & Ch16:66970561 \\
\hline CES2 & N & CES2_c.269-965A>G & AM_11066 & rs4783745 & Ch16:66970975 \\
\hline CES2 & N & CES2_c.269-683G>A & AM_11067 & rs11568311 & Ch16:66971257 \\
\hline CES2 & R98W & CES2_C.292C>T(R98W) & AM_11068 & rs72547531 & Ch16:66971963 \\
\hline CES2 & V206M & CES2_c.616G>A(V206M) & AM_11070 & rs72547532 & Ch16:66974125 \\
\hline CES2 & IVS8 & CES2_c.1330-2A>G(SpliceDefect) & AM_11075 & rs72547533 & Ch16:66976006 \\
\hline CES2 & IVS10 & CES2_c.1613-88C>T(SpliceDefect) & AM_11076 & rs3893757 & Ch16:66977114 \\
\hline CHST7 & $\mathrm{N}$ & CHST7_c.*31+10802G>A & AM_15403 & rs6521128 & ChX:46445660 \\
\hline CHST7 & N & CHST7_c.*32-7531A>C & AM_15404 & rs11796837 & ChX:46449664 \\
\hline CHST7 & N & CHST7_c.*32-2930C>A & AM_15405 & rs12012841 & ChX:46454265 \\
\hline CHST7 & N & CHST7_C.*58G>A(3'UTR) & AM_15406 & rs7056956 & ChX:46457221 \\
\hline CHST7 & $\mathrm{N}$ & CHST7_c.*623A>G(3'UTR) & AM_15407 & rs735716 & ChX:46457786 \\
\hline CHST7 & $\mathrm{N}$ & CHST7_C.*675+785T>G(3'UTR) & AM_15408 & rs732316 & ChX:46458623 \\
\hline
\end{tabular}




\begin{tabular}{|c|c|c|c|c|c|}
\hline \multirow{2}{*}{$\begin{array}{l}\text { Gene } \\
\text { CHST7 }\end{array}$} & \multicolumn{2}{|c|}{$\begin{array}{l}\text { SummaryCommon Name } \\
\text { Flag }\end{array}$} & \multirow{2}{*}{$\begin{array}{l}\text { Probe Set } \\
\text { ID } \\
\text { AM_15409 }\end{array}$} & \multirow{2}{*}{$\begin{array}{l}\text { dbSNP RS } \\
\text { ID } \\
\text { rs12014644 }\end{array}$} & \multirow{2}{*}{$\begin{array}{l}\text { Chrom } \\
\text { Chx:46460615 }\end{array}$} \\
\hline & $\mathrm{N}$ & CHST7_C. *675+2777C $>$ T(3'UTR) & & & \\
\hline CYP1A1 & *6 & CYP1A1*6_1635G>T(M331I) & AM_10774 & rs56313657 & Ch15:75013804 \\
\hline CYP1A1 & *7 & CYP1A1*7_2345insT & AM_10771 & rs72547510 & Ch15:75013093 \\
\hline CYP1A1 & $\star_{8}$ & CYP1A1*8_2413T>A(I448N) & AM_10770 & rs72547509 & Ch15:75013026 \\
\hline CYP1A1 & $*_{4}$ & CYP1A1*4_2452C>A(T461N) & AM_10769 & rs1799814 & Ch15:75012987 \\
\hline CYP1A1 & $\star 2 \mathrm{C}$ & CYP1A1*2C_2454A>G(1462V) & AM_10768 & rs1048943 & Ch15:75012985 \\
\hline CYP1A1 & $\star 5, * 9$ & $\begin{array}{l}\text { CYP1A1*5or*9_2460C }>A>T \\
\text { |(R464SorC) }\end{array}$ & AM_10766 & rs41279188 & $\begin{array}{l}\text { Ch15:75012979, } \\
\text { Ch15:75012979 }\end{array}$ \\
\hline CYP1A1 & *10 & CYP1A1*10_2499C>T(R477W) & AM_10765 & rs56240201 & Ch15:75012940 \\
\hline CYP1A1 & *3 & CYP1A1*3_3204T>C(3'UTR) & AM_10762 & rs1800031 & Ch15:75012235 \\
\hline CYP1A1 & G45D & CYP1A1_134G>A(G45D) & AM_10778 & rs4646422 & Ch15:75015305 \\
\hline CYP1A1 & R279W & CYP1A1_1390C>T(R279W) & AM_10776 & rs34260157 & Ch15:75014049 \\
\hline CYP1A1 & I286T & CYP1A1_1412T>C(I286T) & AM_10775 & rs4987133 & Ch15:75014027 \\
\hline CYP1A1 & F381L & CYP1A1_1876C>A(F381L) & AM_10772 & rs2856833 & Ch15:75013563 \\
\hline CYP1A1 & A463G & CYP1A1_2458C>G(A463G) & AM_10767 & rs2278970 & Ch15:75012981 \\
\hline CYP1A2 & PR & CYP1A2*1C_-3860G>A(Promoter) & AM_10780 & rs2069514 & Ch15:75038220 \\
\hline CYP1A2 & $\star 1 \mathrm{~K}$ & CYP1A2*1K_-729C>T(Promoter) & AM_10784 & rs12720461 & Ch15:75041351 \\
\hline CYP1A2 & PR & CYP1A2*1F_-163C>A(Promoter) & AM_10785 & rs762551 & Ch15:75041917 \\
\hline CYP1A2 & *2 & $\mathrm{CYP} 1 \mathrm{~A} 2 * 2 \_63 \mathrm{C}>\mathrm{G}(\mathrm{F} 21 \mathrm{~L})$ & AM_10787 & rs56160784 & Ch15:75042142 \\
\hline CYP1A2 & *15 & CYP1A2*15_125C>G(P42R) & AM_10788 & rs72547511 & Ch15:75042204 \\
\hline CYP1A2 & $\star 11$ & CYP1A2*11_558C>A(F186L) & AM_10793 & rs72547513 & Ch15:75042637 \\
\hline CYP1A2 & *3 & CYP1A2*3_2116G>A(D348N) & AM_10796 & rs56276455 & Ch15:75044195 \\
\hline CYP1A2 & *16 & CYP1A2*16_2473G>A(R377Q) & AM_10798 & rs72547515 & Ch15:75044552 \\
\hline CYP1A2 & $\star_{4}$ & CYP1A2*4_2499A>T(I386F) & AM_10799 & rs72547516 & Ch15:75044578 \\
\hline CYP1A2 & *5 & CYP1A2*5_3497G>A(C406Y) & AM_10801 & rs55889066 & Ch15:75045575 \\
\hline CYP1A2 & *7 & CYP1A2*7_3533G>A(SpliceDefect) & AM_10802 & rs56107638 & Ch15:75045612 \\
\hline CYP1A2 & *6 & CYP1A2*6_5090C>T(R431W) & AM_10803 & rs28399424 & Ch15:75047169 \\
\hline CYP1A2 & *8 & CYP1A2*8_5166G>A(R456H) & AM_10805 & rs72547517 & Ch15:75047245 \\
\hline CYP1A2 & *1D & CYP1A2*1D_-2467delT(Promoter) & AM_10782 & rs35694136 & Ch15:75039613 \\
\hline CYP1A2 & PR & CYP1A2*1K_-739T>G(Promoter) & AM_10783 & rs2069526 & Ch15:75041341 \\
\hline CYP1A2 & $\mathrm{N}$ & CYP1A2_5347T>C(N516N) & AM_10807 & rs2470890 & Ch15:75047426 \\
\hline CYP1B1 & $\star 12$ & CYP1B1*12_182G>A(G61E) & AM_12493 & rs28936700 & Ch2:38302350 \\
\hline CYP1B1 & *17 & $\begin{array}{l}\text { CYP1B1*17_4096del13 } \\
\text { (RVQAE355X) }\end{array}$ & AM_12473 & rs72549380 & Ch2:38298421 \\
\hline CYP1B1 & *18 & CYP1B1*18_4125G>T(G365W) & AM_12471 & rs55771538 & Ch2:38298404 \\
\hline CYP1B1 & *19 & CYP1B1*19_4168C>T(P379L) & AM_12467 & rs56305281 & Ch2:38298361 \\
\hline CYP1B1 & $\star 20$ & CYP1B1*20_4191G>A(E387K) & AM_12466 & rs55989760 & Ch2:38298338 \\
\hline CYP1B1 & $\star 21$ & CYP1B1*21_4201G>A(R390H) & AM_12465 & rs56010818 & Ch2:38298328 \\
\hline CYP1B1 & *3 & CYP1B1*3_4326C>G(L432V) & AM_12461 & rs1056836 & Ch2:38298203 \\
\hline CYP1B1 & $\star 23$ & CYP1B1*23_4342C>T(P437L) & AM_12460 & rs56175199 & Ch2:38298187 \\
\hline CYP1B1 & $\star 7$ & CYP1B1*7_4360C>G(A443G) & AM_12458 & rs4986888 & Ch2:38298169 \\
\hline CYP1B1 & $\star 24$ & CYP1B1*24_4377delG(D449X) & AM_12456 & rs72549375 & Ch2:38298152 \\
\hline CYP1B1 & $\star_{4}$ & CYP1B1*4_4390A>G(N453S) & AM_12454 & rs1800440 & Ch2:38298139 \\
\hline CYP1B1 & $\star 25$ & CYP1B1*25_4437C>T(R469W) & AM_12452 & rs28936701 & Ch2:38298092 \\
\hline CYP1B1 & N & CYP1B1_81G>C(L27L) & AM_12498 & rs4987135 & Ch2:38302451 \\
\hline
\end{tabular}




\begin{tabular}{|c|c|c|c|c|c|}
\hline Gene & $\begin{array}{l}\text { Summary } \\
\text { Flag }\end{array}$ & Common Name & $\begin{array}{l}\text { Probe Set } \\
\text { ID }\end{array}$ & $\begin{array}{l}\text { dbSNP RS } \\
\text { ID }\end{array}$ & Chrom \\
\hline CYP1B1 & R355X & CYP1B1_4095C>T(R355X) & AM_12474 & rs72549381 & Ch2:38298434 \\
\hline CYP1B1 & M372V & CYP1B1_4146A>G(M372V) & AM_12469 & rs4398252 & Ch2:38298383 \\
\hline CYP1B1 & $\begin{array}{l}\text { N, } \\
\text { D449DorE }\end{array}$ & CYP1B1_4379C>TorA(D449DorE) & AM_12455 & rs1056837 & $\begin{array}{l}\text { Ch2:38298150, } \\
\text { Ch2:38298150 }\end{array}$ \\
\hline CYP1B1 & N & CYP1B1_4424A>T(S464S) & AM_12453 & rs4986889 & Ch2:38298105 \\
\hline CYP2A6 & $\star 9$ & CYP2A6*9_-48T>G(Promoter) & AM_11361 & rs28399433 & Ch19:41356379 \\
\hline CYP2A6 & $\star 13$ & CYP2A6*13_13G>A(G5R) & AM_11360 & rs28399434 & Ch19:41356319 \\
\hline CYP2A6 & $\star_{6} 6$ & CYP2A6*6_1703G>A(R128Q) & AM_11353 & rs4986891 & Ch19:41354629 \\
\hline CYP2A6 & $\star 2$ & CYP2A6*2_1799T>A(L160H) & AM_11351 & rs1801272 & Ch19:41354533 \\
\hline CYP2A6 & $\star 20$ & CYP2A6*20_2141deIAA(K196X) & AM_11346 & rs28399444 & Ch19:41354190 \\
\hline CYP2A6 & $\star 11$ & CYP2A6*11_3391T>C(S224P) & AM_11344 & rs111033610 & Ch19:41352941 \\
\hline CYP2A6 & $\star 17$ & CYP2A6*17_5065G>A(V365M) & AM_11337 & rs28399454 & Ch19:41351267 \\
\hline CYP2A6 & *7 & CYP2A6*7_6558T>C(1471T) & AM_11330 & rs5031016 & Ch19:41349774 \\
\hline CYP2A6 & $\star 28$ & CYP2A6*28_5750G>C(E419D) & AM_11332 & rs8192730 & Ch19:41350582 \\
\hline CYP2A6 & *8 & CYP2A6*8_6600G>T(R485L) & AM_11327 & rs28399468 & Ch19:41349732 \\
\hline CYP2A6 & *1D & CYP2A6*1D_-1013A>G & AM_11364 & rs4803381 & Ch19:41357344 \\
\hline CYP2A6 & $\mathrm{N}$ & CYP2A6_22C>T(L8L) & AM_11359 & rs8192720 & Ch19:41356310 \\
\hline CYP2A6 & $\mathrm{N}$ & CYP2A6_51G>A(V17V) & AM_11358 & rs1137115 & Ch19:41356281 \\
\hline CYP2A6 & N & CYP2A6_1874G>T & AM_11349 & rs28399442 & Ch19:41354458 \\
\hline CYP2A6 & $\mathrm{N}$ & CYP2A6_3420A>G(P233P) & AM_11343 & rs3891219 & Ch19:41352912 \\
\hline CYP2A6 & $\mathrm{N}$ & CYP2A6_3570C>G & AM_11342 & rs4079369 & Ch19:41352762 \\
\hline CYP2A6 & $\mathrm{N}$ & CYP2A6_4365A>G(K289K) & AM_11341 & rs2644905 & Ch19:41351967 \\
\hline CYP2A6 & $387 F S$ & CYP2A6_5132delA(K387X) & AM_11336 & rs72547582 & Ch19:41351200 \\
\hline CYP2A6 & $\mathrm{N}$ & CYP2A6_5336G>A & AM_11334 & rs8192729 & Ch19:41350996 \\
\hline CYP2A13 & $\mathrm{N}$ & CYP2A13_2366C>T & AM_11440 & rs1645691 & Ch19:41596742 \\
\hline CYP2A13 & $\mathrm{N}$ & CYP2A13_6432C>T & AM_11446 & rs1645694 & Ch19:41600808 \\
\hline CYP2A13 & $\mathrm{N}$ & CYP2A13_7233T>G & AM_11449 & rs1709082 & Ch19:41601609 \\
\hline CYP2A13 & P321L & CYP2A13_5289C>T(P321L) & AM_11443 & rs3885816 & Ch19:41599665 \\
\hline CYP2B6 & *22 & CYP2B6*22_-82T>C & AM_11398 & rs34223104 & Ch19:41497129 \\
\hline CYP2B6 & $\star_{2}$ & CYP2B6*2_64C>T(R22C) & AM_11399 & rs8192709 & Ch19:41497274 \\
\hline CYP2B6 & $\star 11$ & CYP2B6*11_136A>G(M46V) & AM_11401 & rs35303484 & Ch19:41497346 \\
\hline CYP2B6 & *12 & CYP2B6*12_12820G>A(G99E) & AM_11403 & rs36060847 & Ch19:41510030 \\
\hline CYP2B6 & $\star 8$ & CYP2B6*8_13072A>G(K139E) & AM_11405 & rs12721655 & Ch19:41510282 \\
\hline CYP2B6 & *14 & CYP2B6*14_13076G>A(R140Q) & AM_11406 & rs35773040 & Ch19:41510286 \\
\hline CYP2B6 & *26 & CYP2B6*26_15614C>G(P167A) & AM_11409 & rs3826711 & Ch19:41512824 \\
\hline CYP2B6 & *20 & CYP2B6*20_15618C>T(T168I) & AM_11410 & rs36056539 & Ch19:41512828 \\
\hline CYP2B6 & $*_{6}$ & CYP2B6*6_15631G>T(Q172H) & AM_11411 & rs3745274 & Ch19:41512841 \\
\hline CYP2B6 & $\star 27$ & CYP2B6*27_15708T>C(M198T) & AM_11413 & rs36079186 & Ch19:41512918 \\
\hline CYP2B6 & *3 & CYP2B6*3_18045C>A(S259R) & AM_11414 & rs45482602 & Ch19:41515255 \\
\hline CYP2B6 & $\star 4$ & CYP2B6*4_18053A>G(K262R) & AM_11415 & rs2279343 & Ch19:41515263 \\
\hline CYP2B6 & $\star 16$ & CYP2B6*16_21011T>C(I328T) & AM_11417 & rs28399499 & Ch19:41518221 \\
\hline CYP2B6 & *19 & CYP2B6*19_21034C>T(R336C) & AM_11418 & rs34826503 & Ch19:41518244 \\
\hline CYP2B6 & $\star 28$ & CYP2B6*28_21160C>T(R378X) & AM_11419 & rs34097093 & Ch19:41518370 \\
\hline CYP2B6 & *15 & CYP2B6*15_21388T>A(I391N) & AM_11420 & rs35979566 & Ch19:41518598 \\
\hline CYP2B6 & $\star 21$ & CYP2B6*21_21498C>A(P428T) & AM_11422 & rs35010098 & Ch19:41518708 \\
\hline
\end{tabular}




\begin{tabular}{|c|c|c|c|c|c|}
\hline \multirow{2}{*}{$\begin{array}{l}\text { Gene } \\
\text { CYP2B6 }\end{array}$} & \multicolumn{2}{|c|}{$\begin{array}{l}\text { Summary Common Name } \\
\text { Flag }\end{array}$} & \multirow{2}{*}{$\begin{array}{l}\text { Probe Set } \\
\text { ID } \\
\text { AM_11426 }\end{array}$} & \multirow{2}{*}{$\begin{array}{l}\text { dbSNP RS } \\
\text { ID } \\
\text { rs3211371 }\end{array}$} & \multirow{2}{*}{$\begin{array}{l}\text { Chrom } \\
\text { Ch19:41522715 }\end{array}$} \\
\hline & $\star 5$ & CYP2B6*5_25505C>T(R487C) & & & \\
\hline CYP2B6 & $\mathrm{N}$ & CYP2B6_12740G>C(P72P) & AM_11402 & rs2279341 & Ch19:41509950 \\
\hline CYP2B6 & $N$ & CYP2B6_14593C>G & AM_11407 & rs4803418 & Ch19:41511803 \\
\hline CYP2B6 & $\mathrm{N}$ & CYP2B6_15582C>T & AM_11408 & rs4803419 & Ch19:41512792 \\
\hline CYP2B6 & $\mathrm{N}$ & CYP2B6_18273G>A & AM_11416 & rs2279344 & Ch19:41515483 \\
\hline CYP2B6 & $N$ & CYP2B6_21563C>T & AM_11423 & rs8192719 & Ch19:41518773 \\
\hline CYP2C8 & *3 & CYP2C8*3_2130G>A(R139K) & AM_10135 & rs11572080 & Ch10:96827030 \\
\hline CYP2C8 & *5 & CYP2C8*5_2189delA(T159X) & AM_10133 & rs72558196 & Ch10:96826971 \\
\hline CYP2C8 & $* 7, * 8$ & $\begin{array}{l}\text { CYP2C8*7or*8_4517C }>T>G \\
\text { (R186XorG) }\end{array}$ & AM_10131 & rs72558195 & $\begin{array}{l}\text { Ch10:96824643, } \\
\text { Ch10:96824643 }\end{array}$ \\
\hline CYP2C8 & $\star_{4}$ & CYP2C8*4_11041C>G(I264M) & AM_10129 & rs1058930 & Ch10:96818119 \\
\hline CYP2C8 & $\star 2$ & CYP2C8*2_11054A>T(I269F) & AM_10128 & rs11572103 & Ch10:96818106 \\
\hline CYP2C8 & *3 & CYP2C8*3_30411A>G(K399R) & AM_10125 & rs10509681 & Ch10:96798749 \\
\hline CYP2C8 & $\star 12$ & $\begin{array}{l}\text { CYP2C8*12_32184_32186delTTG } \\
\text { (V461X) }\end{array}$ & AM_10123 & rs3832694 & Ch10:96796974 \\
\hline CYP2C8 & N & CYP2C8_-86A>C & AM_10138 & rs11572066 & Ch10:96829245 \\
\hline CYP2C8 & L390S & CYP2C8_30384T>C(L390S) & AM_10126 & rs72558194 & Ch10:96798776 \\
\hline CYP2C8 & P404A & CYP2C8_30425C>G(P404A) & AM_10124 & rs66501115 & Ch10:96798735 \\
\hline CYP2C8 & N & CYP2C8_32364C>T(3’UTR) & AM_10122 & rs28399518 & Ch10:96796796 \\
\hline CYP2C9 & *13 & CYP2C9*13_3276T>C(L90P) & AM_10093 & rs72558187 & Ch10:96701715 \\
\hline CYP2C9 & $\star 25$ & CYP2C9*25_3531_3540del10 & AM_10095 & rs72558188 & Ch10:96701970 \\
\hline CYP2C9 & *14 & CYP2C9*14_3552G>A(R125H) & AM_10097 & rs72558189 & Ch10:96701991 \\
\hline CYP2C9 & *2 & CYP2C9*2_3608C>T(R144C) & AM_10100 & rs1799853 & Ch10:96702047 \\
\hline CYP2C9 & *15 & CYP2C9*15_9100C>A(S162x) & AM_10104 & rs72558190 & Ch10:96707539 \\
\hline CYP2C9 & *9 & CYP2C9*9_10535A>G(H251R) & AM_10106 & rs2256871 & Ch10:96708974 \\
\hline CYP2C9 & *10 & CYP2C9*10_10598A>G(E272G) & AM_10107 & rs9332130 & Ch10:96709037 \\
\hline CYP2C9 & *6 & CYP2C9*6_10601delA(K273X) & AM_10108 & rs9332131 & Ch10:96709039 \\
\hline CYP2C9 & *16 & CYP2C9*16_33497A>G(T299A) & AM_10109 & rs72558192 & Ch10:96731936 \\
\hline CYP2C9 & *11 & CYP2C9*11_42542C>T(R335W) & AM_10111 & rs28371685 & Ch10:96740981 \\
\hline CYP2C9 & *3 & CYP2C9*3_42614A>C(I359L) & AM_10113 & rs1057910 & Ch10:96741053 \\
\hline CYP2C9 & *4 & CYP2C9*4_42615T>C(I359T) & AM_10114 & rs56165452 & Ch10:96741054 \\
\hline CYP2C9 & $\star 5$ & CYP2C9*5_42619C>G(D360E) & AM_10115 & rs28371686 & Ch10:96741058 \\
\hline CYP2C9 & *12 & CYP2C9*12_50338C>T(P489S) & AM_10121 & rs9332239 & Ch10:96748777 \\
\hline CYP2C9 & Y358C & CYP2C9_42612A>G(Y358C) & AM_10112 & rs1057909 & Ch10:96741051 \\
\hline CYP2C9 & $\mathrm{N}$ & CYP2C9_55221C>T(A441A) & AM_10119 & rs2017319 & Ch10:96748635 \\
\hline CYP2C9 & $\mathrm{N}$ & CYP2C9_55323A>T(G475G) & AM_10120 & rs1057911 & Ch10:96748737 \\
\hline CYP2C18 & Y68X & CYP2C18_C.204T>A(Y68X) & AM_10039 & rs41291550 & Ch10:96447562 \\
\hline CYP2C18 & $195 F S$ & CYP2C18_c.582insT & AM_10042 & rs72558183 & Ch10:96454774 \\
\hline CYP2C18 & K232E & CYP2C18_C.694A>G(K232E) & AM_10044 & rs2296681 & Ch10:96466592 \\
\hline CYP2C18 & T385M & CYP2C18_C.1154C>T(T385M) & AM_10047 & rs2281891 & Ch10:96493058 \\
\hline CYP2C18 & $\mathrm{N}$ & CYP2C18_C.*31C>T(3'UTR) & AM_10048 & rs2860840 & Ch10:96495232 \\
\hline CYP2C18 & N & CYP2C18_C.*592C>A(3'UTR) & AM_10052 & rs1326830 & Ch10:96495793 \\
\hline CYP2C19 & *17 & CYP2C19*17_-806C>T & AM_10053 & rs12248560 & Ch10:96521657 \\
\hline CYP2C19 & $*_{4}$ & CYP2C19*4_1A>G(M1V) & AM_10054 & rs28399504 & Ch10:96522463 \\
\hline CYP2C19 & *14 & CYP2C19*14_50T>C(L17P) & AM_10055 & rs55752064 & Ch10:96522512 \\
\hline
\end{tabular}




\begin{tabular}{|c|c|c|c|c|c|}
\hline \multirow{2}{*}{$\begin{array}{l}\text { Gene } \\
\text { CYP2C19 }\end{array}$} & \multicolumn{2}{|c|}{$\begin{array}{l}\text { SummaryCommon Name } \\
\text { Flag }\end{array}$} & \multirow{2}{*}{$\begin{array}{l}\text { Probe Set } \\
\text { ID } \\
\text { AM_10056 }\end{array}$} & \multirow{2}{*}{$\begin{array}{l}\text { dbSNP RS } \\
\text { ID } \\
\text { rs17882687 }\end{array}$} & \multirow{2}{*}{$\begin{array}{l}\text { Chrom } \\
\text { Ch10:96522517 }\end{array}$} \\
\hline & $\star 15$ & CYP2C19*15_55A>C(I19L) & & & \\
\hline CYP2C19 & $\star 2 \mathrm{~B}$ & CYP2C19*2B_12460G>C(E92D) & AM_10059 & rs17878459 & Ch10:96534922 \\
\hline CYP2C19 & $\star 8$ & CYP2C19*8_12711T>C(W120R) & AM_10060 & rs41291556 & Ch10:96535173 \\
\hline CYP2C19 & $\star_{6} 6$ & CYP2C19*6_12748G>A(R132Q) & AM_10062 & rs72558184 & Ch10:96535210 \\
\hline CYP2C19 & *9 & CYP2C19*9_12784G>A(R144H) & AM_10064 & rs17884712 & Ch10:96535246 \\
\hline CYP2C19 & *3 & CYP2C19*3_17948G>A(W212X) & AM_10068 & rs4986893 & Ch10:96540410 \\
\hline CYP2C19 & $\star 10$ & CYP2C19*10_19153C>T(P227L) & AM_10069 & rs6413438 & Ch10:96541615 \\
\hline CYP2C19 & $\star_{2}$ & CYP2C19*2_19154G>A(P227P) & AM_10070 & rs4244285 & Ch10:96541616 \\
\hline CYP2C19 & $\star 7$ & $\begin{array}{l}\text { CYP2C19*7_19294T>A } \\
\text { (SpliceDefect) }\end{array}$ & AM_10072 & rs72558186 & Ch10:96541756 \\
\hline CYP2C19 & *13 & CYP2C19*13_87290C>T(R410C) & AM_10077 & rs17879685 & Ch10:96609752 \\
\hline CYP2C19 & $\star 5$ & CYP2C19*5_90033C>T(R433W) & AM_10079 & rs56337013 & Ch10:96612495 \\
\hline CYP2C19 & $\star 12$ & CYP2C19*12_90209A>C(X491C) & AM_10082 & rs55640102 & Ch10:96612671 \\
\hline CYP2C19 & 439FS & CYP2C19_90052delG & AM_10080 & rs5787121 & Ch10:96612514 \\
\hline CYP2C19 & $241 \mathrm{FS}$ & CYP2C19_721insG & AM_10071 & rs72558185 & Ch10:96541656 \\
\hline CYP2C19 & V331I & CYP2C19_80161G>A(V331I) & AM_10075 & rs3758581 & Ch10:96602623 \\
\hline CYP2D6 & P34S & CYP2D6_100C>T(P34S) & AM_12285 & rs1065852 & Ch22:42526694 \\
\hline CYP2D6 & *12 & CYP2D6*12_124G>A(G42R) & AM_12284 & rs5030862 & Ch22:42526670 \\
\hline CYP2D6 & *15 & CYP2D6*15_137insT & AM_12283 & rs72549357 & Ch22:42526657 \\
\hline CYP2D6 & *11 & CYP2D6*11_883G>C(SpliceDefect) & AM_12281 & rs5030863 & Ch22:42525912 \\
\hline CYP2D6 & T1071 & CYP2D6_1023C>T(T107I) & AM_12280 & rs28371706 & Ch22:42525772 \\
\hline CYP2D6 & *29 & CYP2D6*29_1659G>A(V136I) & AM_12278 & rs61736512 & Ch22:42525134 \\
\hline CYP2D6 & $*_{6}$ & CYP2D6*6_1707delT(W152X) & AM_12276 & rs5030655 & Ch22:42525086 \\
\hline CYP2D6 & $\star 14, * 8$ & $\begin{array}{l}\text { CYP2D6*14or*8_1758G>A>T } \\
\text { (G169RorX) }\end{array}$ & AM_12275 & rs5030865 & $\begin{array}{l}\text { Ch22:42525035, } \\
\text { Ch22:42525035 }\end{array}$ \\
\hline CYP2D6 & $\star_{4}$ & CYP2D6*4_1846G>A(SpliceDefect) & AM_12274 & rs3892097 & Ch22:42524947 \\
\hline CYP2D6 & $\star 40$ & CYP2D6*40_1863ins(TTTCGCCCC)2 & AM_12272 & rs72549356 & Ch22:42524929 \\
\hline CYP2D6 & $\star 20$ & CYP2D6*20_1973insG & AM_12270 & rs72549354 & Ch22:42524819 \\
\hline CYP2D6 & $\star 19$ & CYP2D6*19_2539delAACT & AM_12268 & rs72549353 & Ch22:42524251 \\
\hline CYP2D6 & *3 & CYP2D6*3_2549delA(R259X) & AM_12267 & rs35742686 & Ch22:42524244 \\
\hline CYP2D6 & $\star 21$ & CYP2D6*21_2573insC & AM_12266 & rs72549352 & Ch22:42524213 \\
\hline CYP2D6 & *38 & CYP2D6*38_2587delGACT & AM_12265 & rs72549351 & Ch22:42524203 \\
\hline CYP2D6 & *9 & CYP2D6*9_2615delAAG & AM_12264 & rs28371720 & Ch22:42524178 \\
\hline CYP2D6 & R296C & CYP2D6_2850C>T(R296C) & AM_12261 & rs16947 & Ch22:42523943 \\
\hline CYP2D6 & $\star 7$ & CYP2D6*7_2935A>C(H324P) & AM_12259 & rs5030867 & Ch22:42523858 \\
\hline CYP2D6 & *44 & CYP2D6*44_2950G>C(SpliceDefect) & AM_12258 & rs72549349 & Ch22:42523843 \\
\hline CYP2D6 & $\star 41$ & CYP2D6*41_2988G>A(SpliceDefect) & AM_12257 & rs28371725 & Ch22:42523805 \\
\hline CYP2D6 & $\star 29$ & CYP2D6*29_3183G>A(V338M) & AM_12255 & rs59421388 & Ch22:42523610 \\
\hline CYP2D6 & *56 & CYP2D6*56_3201C>T(R344X) & AM_12254 & rs72549347 & Ch22:42523592 \\
\hline CYP2D6 & *42 & CYP2D6*42_3259insGT & AM_12252 & rs72549346 & Ch22:42523533 \\
\hline CYP2D6 & *18 & CYP2D6*18_4125dupGTGCCCACT & AM_12248 & rs1135836 & Ch22:42522660 \\
\hline CYP2D6 & N & CYP2D6_-2178G>A & AM_15506 & rs28360521 & Ch22:42528976 \\
\hline CYP2D6 & N & CYP2D6_-1961C>G>A & AM_15503 & N/A & $\begin{array}{l}\text { Ch22:42528759, } \\
\text { Ch22:42528759 }\end{array}$ \\
\hline CYP2D6 & $\mathrm{N}$ & CYP2D6_-1770G>A & AM_15502 & rs1080983 & Ch22:42528568 \\
\hline
\end{tabular}




\begin{tabular}{|c|c|c|c|c|c|}
\hline \multirow{2}{*}{$\begin{array}{l}\text { Gene } \\
\text { CYP2D6 }\end{array}$} & \multicolumn{2}{|c|}{$\begin{array}{l}\text { Summary Common Name } \\
\text { Flag }\end{array}$} & \multirow{2}{*}{$\begin{array}{l}\text { Probe Set } \\
\text { ID } \\
\text { AM_12291 }\end{array}$} & \multirow{2}{*}{$\begin{array}{l}\text { dbSNP RS } \\
\text { ID } \\
\text { rs1080985 }\end{array}$} & \multirow{2}{*}{$\begin{array}{l}\text { Chrom } \\
\text { Ch22:42528382 }\end{array}$} \\
\hline & $N$ & CYP2D6_-1584C>G & & & \\
\hline CYP2D6 & $N$ & CYP2D6_1661G>C(V136V) & AM_12277 & rs1058164 & Ch22:42525132 \\
\hline CYP2D6 & S486T & CYP2D6_4180G>C(S486T) & AM_12247 & rs1135840 & Ch22:42522613 \\
\hline CYP2E1 & $\star 5$ & CYP2E1*5_-1293G>C(Promoter) & AM_10240 & rs3813867 & Ch10:135339605 \\
\hline CYP2E1 & *5 & CYP2E1*5_-1053C> T(Promoter) & AM_10241 & rs2031920 & Ch10:135339845 \\
\hline CYP2E1 & $* 7 C$ & CYP2E1*7C_-352A>G(Promoter) & AM_10242 & rs2070672 & Ch10:135340548 \\
\hline CYP2E1 & *7 & CYP2E1*7_-333T>A(Promoter) & AM_10243 & rs2070673 & Ch10:135340567 \\
\hline CYP2E1 & $\star 7 \mathrm{~B}$ & CYP2E1*7B_-71G>T(Promoter) & AM_10244 & rs6413420 & Ch10:135340829 \\
\hline CYP2E1 & *2 & CYP2E1*2_1132G>A(R76H) & AM_10249 & rs72559710 & Ch10:135342034 \\
\hline CYP2E1 & *4 & CYP2E1*4_4768G>A(V179I) & AM_10251 & rs6413419 & Ch10:135345675 \\
\hline CYP2E1 & *3 & CYP2E1*3_10023G>A(V389I) & AM_10257 & rs55897648 & Ch10:135351264 \\
\hline CYP2E1 & N & CYP2E1_6498C>T(I321I) & AM_10253 & rs915909 & Ch10:135347397 \\
\hline CYP2E1 & N & CYP2E1_10463T>C(F421F) & AM_10258 & rs2515641 & Ch10:135351362 \\
\hline CYP2F1 & N & CYP2F1_96G>A(P32P) & AM_11456 & rs305968 & Ch19:41622189 \\
\hline CYP2F1 & *4 & CYP2F1*4_112T>C(S38P) & AM_11457 & rs58285195 & Ch19:41622205 \\
\hline CYP2F1 & *6 & CYP2F1*6_388G>C(R98P) & & rs57670668 & Ch19:41622481 \\
\hline CYP2F1 & *3 & CYP2F1*3_11887C>T(P490L) & AM_11462 & rs7246981 & Ch19:41633980 \\
\hline CYP2F1 & V175L & CYP2F1_5308G>C(V175L) & AM_11459 & & Ch19:41627401 \\
\hline CYP2J2 & $\star 7$ & CYP2J2*7_-76G>T(Promoter) & & & Ch1:60392494 \\
\hline CYP2J2 & $*_{2}$ & CYP2J2*2_14488A>G(T143A) & AM_11630 & rs55753213 & Ch1:60377930 \\
\hline CYP2J2 & *3 & CYP2J2*3_14533C >T(R158C) & AM_11629 & rs56307989 & Ch1:60377885 \\
\hline CYP2J2 & $\star_{4}$ & CYP2J2*4_15029T>A(I192N) & AM_11627 & rs66515830 & Ch1:60377389 \\
\hline CYP2J2 & *5 & CYP2J2*5_21709G $>A(D 342 N)$ & AM_11625 & rs56053398 & Ch1:60370710 \\
\hline CYP2J2 & $*_{6}$ & CYP2J2*6_25662A>T(N404Y) & AM_11622 & rs72547598 & Ch1:60366757 \\
\hline CYP2J2 & N & CYP2J2_10769C>G(R111R) & AM_11632 & rs1056595 & Ch1:60381650 \\
\hline CYP2J2 & L378Q & CYP2J2_21818T>A(L378Q) & AM_11624 & rs1056596 & Ch1:60370601 \\
\hline CYP2J2 & N & CYP2J2_33084T>A(V499V) & AM_11621 & rs2228114 & Ch1:60359335 \\
\hline CYP2S1 & N & CYP2S1_1300G>A(P66P) & AM_11463 & rs60694775 & Ch19:41700469 \\
\hline CYP2S1 & N & CYP2S1_1324C>G(P74P) & AM_11464 & rs338599 & Ch19:41700493 \\
\hline CYP2S1 & N & CYP2S1_4624G>A(A151A) & AM_11466 & rs57266494 & Ch19:41703793 \\
\hline CYP2S1 & N & CYP2S1_4594G>A(G141G) & AM_11465 & rs16975056 & Ch19:41703763 \\
\hline CYP3A4 & *14 & CYP3A4*14_44T >C(L15P) & AM_14835 & rs12721634 & Ch7:99381661 \\
\hline CYP3A4 & *7 & CYP3A4*7_6004G>A(G56D) & AM_14834 & rs56324128 & Ch7:99375702 \\
\hline CYP3A4 & $\star_{4}$ & CYP3A4*4_13871A>G(I118V) & AM_14831 & rs55951658 & Ch7:99367825 \\
\hline CYP3A4 & *8 & CYP3A4*8_13908G>A(R130Q) & AM_14830 & rs72552799 & Ch7:99367788 \\
\hline CYP3A4 & *15 & CYP3A4*15_14269G>A(R162Q) & AM_14828 & rs4986907 & Ch7:99367427 \\
\hline CYP3A4 & $\star 10$ & CYP3A4*10_14304G>C(D174H) & AM_14826 & rs4986908 & Ch7:99367392 \\
\hline CYP3A4 & $\star 16$ & CYP3A4*16_15603C>G(T185S) & AM_14825 & rs12721627 & Ch7:99366093 \\
\hline CYP3A4 & $\star 17$ & CYP3A4*17_15615T>C(F189S) & AM_14824 & rs4987161 & Ch7:99366081 \\
\hline CYP3A4 & *5 & CYP3A4*5_15702C>G(P218R) & AM_14821 & rs55901263 & Ch7:99365994 \\
\hline CYP3A4 & $\star 2$ & CYP3A4*2_15713T>C(S222P) & AM_14820 & rs55785340 & Ch7:99365983 \\
\hline CYP3A4 & $*_{6}$ & CYP3A4*6_17661 insA & AM_14816 & rs4646438 & Ch7:99364034 \\
\hline CYP3A4 & $\star 18$ & CYP3A4*18_20070T>C(L293P) & AM_14815 & rs28371759 & Ch7:99361626 \\
\hline CYP3A4 & $\star 11$ & CYP3A4*11_21867C>T(T363M) & AM_14812 & rs67784355 & Ch7:99359829 \\
\hline CYP3A4 & *12 & CYP3A4*12_21896C>T(L373F) & AM_14811 & rs12721629 & Ch7:99359800 \\
\hline
\end{tabular}




\begin{tabular}{|c|c|c|c|c|c|}
\hline \multirow{2}{*}{$\begin{array}{l}\text { Gene } \\
\text { CYP3A4 }\end{array}$} & \multicolumn{2}{|c|}{$\begin{array}{l}\text { Summary Common Name } \\
\text { Flag }\end{array}$} & \multirow{2}{*}{$\begin{array}{l}\text { Probe Set } \\
\text { ID } \\
\text { AM_14809 }\end{array}$} & \multirow{2}{*}{$\begin{array}{l}\text { dbSNP RS } \\
\text { ID } \\
\text { rs4986909 }\end{array}$} & \multirow{2}{*}{$\begin{array}{l}\text { Chrom } \\
\text { Ch7:99359670 }\end{array}$} \\
\hline & *13 & CYP3A4*13_22026C>T(P416L) & & & \\
\hline CYP3A4 & *3 & CYP3A4*3_23171T>C(M445T) & AM_14807 & rs4986910 & Ch7:99358524 \\
\hline CYP3A4 & *19 & CYP3A4*19_23237C>T(P467S) & AM_14804 & rs4986913 & Ch7:99358459 \\
\hline CYP3A4 & *20 & CYP3A4*20_25889insA & AM_14802 & rs67666821 & Ch7:99355806 \\
\hline CYP3A4 & $\mathrm{N}$ & CYP3A4_-392A>G & AM_14846 & rs2740574 & Ch7:99382096 \\
\hline CYP3A4 & K96E & CYP3A4_11451A>G(K96E) & AM_14832 & rs3091339 & Ch7:99370245 \\
\hline CYP3A4 & I193V & CYP3A4_15626A>G(1193V) & AM_14823 & rs3208361 & Ch7:99366070 \\
\hline CYP3A4 & S252A & CYP3A4_16898T>G(S252A) & AM_14817 & rs3208363 & Ch7:99364798 \\
\hline CYP3A4 & $\mathrm{N}$ & CYP3A4_20230G>A & AM_14814 & rs2242480 & Ch7:99361466 \\
\hline CYP3A4 & 1431T & CYP3A4_23130T>C(1431T) & AM_14808 & rs1041988 & Ch7:99358566 \\
\hline CYP3A4 & $465 F S$ & CYP3A4_23231insC & AM_14805 & rs72552795 & Ch7:99358465 \\
\hline CYP3A5 & *8 & CYP3A5*8_3699C>T(R28C) & AM_14765 & rs55817950 & Ch7:99273821 \\
\hline CYP3A5 & $* 3 B$ & CYP3A5*3B_3705C>T(H30Y) & AM_14764 & rs28383468 & Ch7:99273815 \\
\hline CYP3A5 & $* 3 \mathrm{~L}$ & CYP3A5*3L_3775A>G(Y53C) & AM_14762 & rs72552791 & Ch7:99273745 \\
\hline CYP3A5 & *3 & CYP3A5*3_6986A>G(SpliceDefect) & AM_14759 & rs776746 & Ch7:99270539 \\
\hline CYP3A5 & $\star 3 D$ & CYP3A5*3D_7249T>G(L82R) & AM_14757 & rs56244447 & Ch7:99270276 \\
\hline CYP3A5 & *5 & CYP3A5*5_12952T>C(SpliceDefect) & AM_14753 & rs55965422 & Ch7:99264573 \\
\hline CYP3A5 & *4 & CYP3A5*4_14665A>G(Q200R) & AM_14749 & rs56411402 & Ch7:99262860 \\
\hline CYP3A5 & *6 & CYP3A5*6_14690G>A(SpliceDefect) & AM_14748 & rs10264272 & Ch7:99262835 \\
\hline CYP3A5 & *9 & CYP3A5*9_19386G>A(A337T) & AM_14739 & rs28383479 & Ch7:99258139 \\
\hline CYP3A5 & *7 & CYP3A5*7_27131insT & AM_14737 & rs41303343 & Ch7:99250393 \\
\hline CYP3A5 & *2 & CYP3A5*2_27289C>A(T398N) & AM_14735 & rs28365083 & Ch7:99250236 \\
\hline CYP3A5 & *3K & CYP3A5*3K_29753T>C(F446S) & AM_14730 & rs41279854 & Ch7:99247772 \\
\hline CYP3A5 & *3F & CYP3A5*3F_31551T>C(1488T) & AM_14726 & rs28365085 & Ch7:99245974 \\
\hline CYP3A5 & S100Y & CYP3A5_7303C>A(S100Y) & AM_14756 & rs41279857 & Ch7:99270222 \\
\hline CYP3A7 & *1B & CYP3A7*1B_-314C>T(Promoter) & AM_14798 & rs45465393 & Ch7:99333030 \\
\hline CYP3A7 & *1C & CYP3A7*1C_-284T>A(Promoter) & AM_14796 & rs45494802 & Ch7:99333000 \\
\hline CYP3A7 & *1C & CYP3A7*1C_-281A>T(Promoter) & AM_14794 & rs45467892 & Ch7:99332997 \\
\hline CYP3A7 & *1C & CYP3A7*1C_-232A>C(Promoter) & AM_14791 & rs45446698 & Ch7:99332948 \\
\hline CYP3A7 & *1D & CYP3A7*1D_-91G>A(Promoter) & AM_14790 & rs55798860 & Ch7:99332807 \\
\hline CYP3A7 & *1E & CYP3A7*1E_-49G>A(Promoter) & AM_14789 & rs28451617 & Ch7:99332765 \\
\hline CYP3A7 & *2 & CYP3A7*2_26041C>G(T409R) & AM_14781 & rs2257401 & Ch7:99306685 \\
\hline CYP3A43 & *3 & CYP3A43*3_31867C>G(P340A) & AM_14861 & rs680055 & Ch7:99457605 \\
\hline CYP3A43 & $\star 1 \mathrm{~B}$ & CYP3A43*1B_33518C>T(A349A) & AM_14862 & rs17342647 & Ch7:99459256 \\
\hline CYP3A43 & N & CYP3A43_14956C>T & AM_14856 & rs533486 & Ch7:99440694 \\
\hline CYP3A43 & N & CYP3A43_19394C>T(L114L) & AM_14857 & rs4646474 & Ch7:99445132 \\
\hline CYP3A43 & M145I & CYP3A43_20053G>T(M145I) & AM_14858 & rs45450092 & Ch7:99445791 \\
\hline CYP3A43 & $\mathrm{N}$ & CYP3A43_21503T>C(N198N) & AM_14859 & rs800667 & Ch7:99447241 \\
\hline CYP3A43 & M275I & CYP3A43_28744G>A(M275I) & AM_14860 & rs45621431 & Ch7:99454482 \\
\hline CYP4B1 & R173W & CYP4B1_C.517C>T(R173W) & AM_11543 & rs4646487 & Ch1:47279175 \\
\hline CYP4B1 & 294FS & CYP4B1_c.881_882delAT(D294X) & AM_11546 & rs3215983 & Ch1:47280747 \\
\hline CYP4B1 & *4 & CYP4B1*4_c.964A>G(S322G) & AM_11547 & rs45467195 & Ch1:47280830 \\
\hline CYP4B1 & M331I & CYP4B1_C.993G>A(M331I) & AM_11549 & rs2297810 & Ch1:47280859 \\
\hline CYP4B1 & R340C & CYP4B1_C.1018C>T(R340C) & AM_11550 & rs4646491 & Ch1:47280884 \\
\hline CYP4B1 & $\mathrm{N}$ & CYP4B1_c.330G>A(K110K) & AM_11539 & rs7513658 & Ch1:47276819 \\
\hline
\end{tabular}




\begin{tabular}{|c|c|c|c|c|c|}
\hline \multirow{2}{*}{$\begin{array}{l}\text { Gene } \\
\text { CYP4B1 }\end{array}$} & \multicolumn{2}{|c|}{$\begin{array}{l}\text { SummaryCommon Name } \\
\text { Flag }\end{array}$} & \multirow{2}{*}{$\begin{array}{l}\text { Probe Set } \\
\text { ID } \\
\text { AM_11554 }\end{array}$} & \multirow{2}{*}{$\begin{array}{l}\text { dbSNP RS } \\
\text { ID } \\
\text { rs2297809 }\end{array}$} & \multirow{2}{*}{$\begin{array}{l}\text { Chrom } \\
\text { Ch1:47282772 }\end{array}$} \\
\hline & R375C & CYP4B1_C.1123C>T(R375C) & & & \\
\hline CYP4F2 & *2 & CYP4F2*2_34T>G(W12G) & AM_11310 & rs3093105 & Ch19:16008388 \\
\hline CYP4F2 & *3 & CYP4F2*3_18000G>A(V433M) & AM_11299 & rs2108622 & Ch19:15990431 \\
\hline CYP4F2 & W12C & CYP4F2_36G>C(W12C) & AM_11309 & rs2906891 & Ch19:16008386 \\
\hline CYP4F2 & P13R & CYP4F2_38C>G(P13R) & AM_11308 & rs2906890 & Ch19:16008384 \\
\hline CYP4F2 & $N$ & CYP4F2_165A>G(P55P) & AM_11307 & rs3093106 & Ch19:16008257 \\
\hline CYP4F2 & N & CYP4F2_2042A>C(G93G) & AM_11305 & rs8100960 & Ch19:16006380 \\
\hline CYP4F2 & N & CYP4F2_2099C>T(N112N) & AM_11304 & rs8110714 & Ch19:16006323 \\
\hline CYP4F2 & N & CYP4F2_5034C>G(A116A) & AM_11303 & rs3093136 & Ch19:16003388 \\
\hline CYP4F2 & G185V & CYP4F2_7207G>T(G185V) & AM_11302 & rs3093153 & Ch19:16001215 \\
\hline CYP4F2 & L278F & CYP4F2_8103C>T(L278F) & AM_11301 & rs4605294 & Ch19:16000319 \\
\hline CYP4F2 & N & CYP4F2_11602C $>\mathrm{T}(\mathrm{H} 343 \mathrm{H})$ & AM_11300 & rs2074900 & Ch19:15996820 \\
\hline CYP17A1 & N & CYP17A1_-34T>C & AM_10206 & rs743572 & Ch10:104597152 \\
\hline CYP17A1 & $\mathrm{C} 22 \mathrm{~W}$ & CYP17A1_66C>G(C22W) & AM_10203 & rs762563 & Ch10:104597053 \\
\hline CYP17A1 & N & CYP17A1_138C > $(\mathrm{H} 46 \mathrm{H})$ & AM_10202 & rs6162 & Ch10:104596981 \\
\hline CYP17A1 & N & CYP17A1_195G>T(S65S) & AM_10201 & & Ch10:104596924 \\
\hline CYP17A1 & N & CYP17A1_4249C>T(D283D) & & rs1042386 & Ch10:104592870 \\
\hline CYP17A1 & N & CYP17A1_6417G>A(P428P) & AM_10194 & & Ch10:104590702 \\
\hline CYP19A1 & $*_{2}$ & CYP19A1*2_115T>C(W39R) & AM_10749 & rs2236722 & Ch15:51534995 \\
\hline CYP19A1 & *4 & CYP19A1*4_27142C>T(R264C) & AM_10747 & rs700519 & Ch15:51507968 \\
\hline CYP19A1 & N & CYP19A1_32124C>T(3'UTR) & AM_10744 & rs10046 & Ch15:51502986 \\
\hline CYP19A1 & N & CYP19A1_32266G>T(3'UTR) & AM_10742 & rs4646 & Ch15:51502844 \\
\hline CYP19A1 & N & CYP19A1_-12829G>C(5'UTR) & AM_10750 & rs1062033 & Ch15:51547938 \\
\hline CYP19A1 & N & CYP19A1_5998A>G(V80V) & AM_10748 & rs700518 & Ch15:51529112 \\
\hline CYP19A1 & $\mathrm{R} 264 \mathrm{H}$ & CYP19A1_27143G>A(R264H) & AM_10746 & rs2304462 & Ch15:51507967 \\
\hline CYP19A1 & N & CYP19A1_30554C>T(P408P) & AM_10745 & rs2304461 & Ch15:51504556 \\
\hline CYP19A1 & N & CYP19A1_32217C>A(3'UTR) & AM_10743 & rs1050677 & Ch15:51502893 \\
\hline CYP19A1 & N & CYP19A1_32812T>C(3'UTR) & AM_10741 & rs1050760 & Ch15:51502298 \\
\hline CYP19A1 & N & CYP19A1_33028T>A(3'UTR) & AM_10740 & rs1050787 & Ch15:51502082 \\
\hline DCK & $124 \mathrm{~V}$ & DCK_c.70A>G(I24V) & AM_13643 & rs66878317 & Ch4:71859622 \\
\hline DCK & A119G & DCK_C.356C>G(A119G) & AM_13644 & rs66472932 & Ch4:71888232 \\
\hline DCK & P122S & DCK_C.364C>T(P122S) & AM_13645 & rs67437265 & Ch4:71888240 \\
\hline DCK & N & DCK_C. ${ }^{*} 165 C>T\left(3^{\prime} U T R\right)$ & AM_13646 & rs4643786 & Ch4:71895260 \\
\hline DPYD & *9 & DPYD*9_c.85T>C(C29R) & AM_11669 & rs1801265 & Ch1:98348885 \\
\hline DPYD & $\star 7$ & DPYD*7_c.295delTCAT & AM_11668 & rs72549309 & Ch1:98205971 \\
\hline DPYD & *8 & DPYD*8_c.703C>T(R235W) & AM_11663 & rs1801266 & Ch1:98157332 \\
\hline DPYD & $\star 11$ & DPYD*11_c.1003G>T(V335L) & AM_11660 & rs72549306 & Ch1:98058899 \\
\hline DPYD & $*_{4}$ & DPYD*4_c.1601G>A(S534N) & AM_11653 & rs1801158 & Ch1:97981421 \\
\hline DPYD & *13 & DPYD*13_c.1679T>G(I560S) & AM_11651 & rs55886062 & Ch1:97981343 \\
\hline DPYD & *3 & DPYD*3_c.1897delC(P633X) & AM_11649 & rs72549303 & Ch1:97915622 \\
\hline DPYD & $\star 2$ & DPYD*2_c.1905+1G>A & AM_11647 & rs3918290 & Ch1:97915614 \\
\hline DPYD & $\star 9 B$ & DPYD*9B_c.2657G>A(R886H) & AM_11643 & rs1801267 & Ch1:97564154 \\
\hline DPYD & $\star 10$ & DPYD*10_c.2983G>T(V995F) & AM_11639 & rs1801268 & Ch1:97544627 \\
\hline DPYD & $\mathrm{R} 21 \mathrm{X}$ & DPYD_c.61C>T(R21X) & AM_11670 & rs72549310 & Ch1:98348909 \\
\hline DPYD & M166V & DPYD_c.496A>G(M166V) & AM_11667 & rs2297595 & Ch1:98165091 \\
\hline
\end{tabular}




\begin{tabular}{|c|c|c|c|c|c|}
\hline \multirow{2}{*}{$\begin{array}{l}\text { Gene } \\
\text { DPYD }\end{array}$} & \multicolumn{2}{|c|}{$\begin{array}{l}\text { SummaryCommon Name } \\
\text { Flag }\end{array}$} & \multirow{2}{*}{$\begin{array}{l}\text { Probe Set } \\
\text { ID } \\
\text { AM_11666 }\end{array}$} & \multirow{2}{*}{$\begin{array}{l}\text { dbSNP RS } \\
\text { ID } \\
\text { rs6670886 }\end{array}$} & \multirow{2}{*}{$\begin{array}{l}\text { Chrom } \\
\text { Ch1:98165062 }\end{array}$} \\
\hline & $N$ & DPYD_c.525G>A(S175S) & & & \\
\hline DPYD & $N$ & DPYD_c.756T>C(G252G) & AM_11662 & rs6675198 & Ch1:98157279 \\
\hline DPYD & $N$ & DPYD_c.1035T>C(F345F) & AM_11659 & rs1042478 & Ch1:98058867 \\
\hline DPYD & $N$ & DPYD_c.1074T>A(R358R) & AM_11658 & rs1042479 & Ch1:98058828 \\
\hline DPYD & N & DPYD_C.1896T>C(F632F) & AM_11650 & rs17376848 & Ch1:97915624 \\
\hline DPYD & $N$ & DPYD_c.1905C>T(N635N) & AM_11648 & rs3918289 & Ch1:97915615 \\
\hline FAAH & Р129T & FAAH_C.385C>A(P129T) & AM_11525 & rs324420 & Ch1:46870761 \\
\hline FAAH & H370R & FAAH_c.1109A>G(H370R) & AM_11526 & rs12094805 & Ch1:46874804 \\
\hline $\mathrm{FMO} 2$ & 113FS & FMO2_c.337delG(V113X) & AM_11933 & rs28369860 & Ch1:171165803 \\
\hline $\mathrm{FMO} 2$ & N413K & FMO2_c.1239T>G(N413K) & AM_11948 & rs2020865 & Ch1:171176912 \\
\hline $\mathrm{FMO} 2$ & X472Q & FMO2_23238T>C(X472Q) & AM_11958 & rs6661174 & Ch1:171178090 \\
\hline $\mathrm{FMO} 2$ & D36G & FMO2_c.107A>G(D36G) & AM_11925 & rs2020870 & Ch1:171154959 \\
\hline $\mathrm{FMO} 2$ & V59l & FMO2_c.175G>A(V59I) & AM_11927 & rs55708639 & Ch1:171162516 \\
\hline $\mathrm{FMO} 2$ & $70 \operatorname{lnsD}$ & FMO2_c.210_211insGAC(70InsD) & AM_11929 & rs2020868 & Ch1:171162551 \\
\hline $\mathrm{FMO} 2$ & F81S & FMO2_C.242T>C(F81S) & AM_11931 & rs2020860 & Ch1:171162583 \\
\hline $\mathrm{FMO} 2$ & F182S & FMO2_C.545T>C(F182S) & AM_11936 & rs2307492 & Ch1:171168545 \\
\hline $\mathrm{FMO} 2$ & N & FMO2_C.585A>G(S195S) & AM_11938 & rs2020861 & Ch1:171168585 \\
\hline $\mathrm{FMO} 2$ & $\mathrm{R} 238 \mathrm{Q}$ & FMO2_c.713G>A(R238Q) & AM_11940 & rs28369895 & Ch1:171173089 \\
\hline $\mathrm{FMO} 2$ & R249X & FMO2_c.745C>T(R249X) & & rs2020866 & Ch1:171173121 \\
\hline $\mathrm{FMO} 2$ & E314G & FMO2_C.941A>G(E314G) & AM_11942 & rs2020863 & Ch1:171174531 \\
\hline $\mathrm{FMO} 2$ & N & FMO2_c.1101G>A(A367A) & AM_11943 & rs7536646 & Ch1:171174691 \\
\hline $\mathrm{FMO} 2$ & R387ins8 & FMO2_c.1160_1161ins8(R387X) & AM_11944 & rs72549336 & Ch1:171174750 \\
\hline $\mathrm{FMO} 2$ & R391T & FMO2_c.1172G>C(R391T) & AM_11945 & rs28369899 & Ch1:171174762 \\
\hline $\mathrm{FMO} 2$ & N & FMO2_c.1206G>A(E402E) & AM_11947 & rs6671692 & Ch1:171176879 \\
\hline $\mathrm{FMO} 2$ & N & FMO2_c. ${ }^{*} 60 \mathrm{~A}>\mathrm{G}$ & AM_11959 & rs2020869 & Ch1:171178152 \\
\hline $\mathrm{FMO} 2$ & N & FMO2_c.*113_*114insT & AM_11960 & rs72549337 & Ch1:171178205 \\
\hline $\mathrm{FMO} 2$ & N & FMO2_c.*933T $>C$ & AM_11966 & rs6664553 & Ch1:171179025 \\
\hline $\mathrm{FMO} 2$ & N & FMO2_C.*1195C>T & AM_11968 & rs7512785 & Ch1:171179287 \\
\hline $\mathrm{FMO} 2$ & N & FMO2_C.*1385C>T & AM_11970 & rs7515157 & Ch1:171179477 \\
\hline FMO3 & E32K & FMO3_c.94G>A(E32K) & AM_11857 & rs72549320 & Ch1:171061893 \\
\hline FMO3 & A52T & FMO3_C.154G>A(A52T) & AM_11866 & rs72549321 & Ch1:171072947 \\
\hline $\mathrm{FMO} 3$ & N61S & FMO3_c.182A>G(N61S) & AM_11867 & rs72549322 & Ch1:171072975 \\
\hline $\mathrm{FMO} 3$ & M66l & FMO3_C.198G>T(M66I) & AM_11868 & rs72549323 & Ch1:171072991 \\
\hline $\mathrm{FMO} 3$ & M82T & FMO3_c.245T>C(M82T) & AM_11869 & rs72549324 & Ch1:171073038 \\
\hline FMO3 & $\mathrm{D} 132 \mathrm{H}$ & FMO3_c.394G>C(D132H) & AM_11871 & rs12072582 & Ch1:171076888 \\
\hline $\mathrm{FMO3}$ & N & FMO3_c.441C>T(S147S) & AM_11872 & rs1800822 & Ch1:171076935 \\
\hline $\mathrm{FMO} 3$ & G148X & FMO3_c.442G>T(G148X) & AM_11873 & rs72549325 & Ch1:171076936 \\
\hline $\mathrm{FMO} 3$ & P153L & FMO3_c.458C>T(P153L) & AM_11874 & rs72549326 & Ch1:171076952 \\
\hline $\mathrm{FMO} 3$ & E158K & FMO3_c.472G>A(E158K) & AM_11875 & rs2266782 & Ch1:171076966 \\
\hline $\mathrm{FMO3}$ & $1199 N$ & FMO3_c.596T>A(I199N) & AM_11877 & rs72549327 & Ch1:171077331 \\
\hline $\mathrm{FMO3}$ & 202FS & FMO3_c.604_605insT(E202X) & AM_11878 & rs72549328 & Ch1:171077339 \\
\hline $\mathrm{FMO3}$ & N & FMO3_c.627+10C>G & AM_11880 & rs2066534 & Ch1:171077372 \\
\hline $\mathrm{FMO3}$ & N & FMO3_c.717T>C(F239F) & AM_11884 & rs1050902 & Ch1:171080028 \\
\hline FMO3 & V257M & FMO3_c.769G >A(V257M) & AM_11885 & rs1736557 & Ch1:171080080 \\
\hline $\mathrm{FMO} 3$ & V277A & FMO3_c.830T>C(V277A) & AM_11887 & rs2066530 & Ch1:171083149 \\
\hline
\end{tabular}




\begin{tabular}{|c|c|c|c|c|c|}
\hline \multirow{2}{*}{$\begin{array}{l}\text { Gene } \\
\text { FMO3 }\end{array}$} & \multicolumn{2}{|c|}{$\begin{array}{l}\text { SummaryCommon Name } \\
\text { Flag }\end{array}$} & \multirow{2}{*}{$\begin{array}{l}\text { Probe Set } \\
\text { ID } \\
\text { AM_11888 }\end{array}$} & \multirow{2}{*}{$\begin{array}{l}\text { dbSNP RS } \\
\text { ID } \\
\text { rs909530 }\end{array}$} & \multirow{2}{*}{$\begin{array}{l}\text { Chrom } \\
\text { Ch1:171083174 }\end{array}$} \\
\hline & $\mathrm{N}$ & FMO3_c.855C>T(N285N) & & & \\
\hline FMO3 & $\mathrm{N}$ & FMO3_c.906C>T(N302N) & AM_11889 & rs2066536 & Ch1:171083225 \\
\hline $\mathrm{FMO3}$ & E305X & FMO3_c.913G>T(E305X) & AM_11890 & rs61753344 & Ch1:171083232 \\
\hline FMO3 & E308G & FMO3_c.923A>G(E308G) & AM_11891 & rs2266780 & Ch1:171083242 \\
\hline $\mathrm{FMO3}$ & E314X & FMO3_c.940G>T(E314X) & AM_11892 & rs72549330 & Ch1:171083259 \\
\hline $\mathrm{FMO3}$ & L360P & FMO3_C.1079T>C(L360P) & AM_11893 & rs28363581 & Ch1:171083398 \\
\hline FMO3 & E362Q & FMO3_C.1084G>C(E362Q) & AM_11894 & rs2066532 & Ch1:171083403 \\
\hline FMO3 & R387L & FMO3_c.1160G>T(R387L) & AM_11895 & rs72549331 & Ch1:171083479 \\
\hline FMO3 & I486M & FMO3_C.1458A>G(1486M) & AM_11902 & rs1050906 & Ch1:171086441 \\
\hline $\mathrm{FMO3}$ & R492W & FMO3_c.1474C $>T(R 492 W)$ & AM_11903 & rs72549334 & Ch1:171086457 \\
\hline $\mathrm{FMO3}$ & G503R & FMO3_c.1507G>A(G503R) & AM_11905 & rs72549335 & Ch1:171086490 \\
\hline GSTM1 & $*_{B}$ & GSTM1*B_C.519G $>C(K 173 N)$ & AM_11707 & rs74837985 & Ch1:110233138 \\
\hline GSTM1 & N & GSTM1_C.178-78T>C & AM_11705 & rs737497 & Ch1:110231592 \\
\hline GSTP1 & $*_{B}$ & GSTP1*B_c.313A>G(1105V) & AM_10440 & rs1695 & Ch11:67352689 \\
\hline GSTP1 & ${ }^{*} \mathrm{C}$ & GSTP1 ${ }^{* C}$ C_C.341C $>$ T(A114V) & AM_10442 & rs1138272 & Ch11:67353579 \\
\hline GSTP1 & N & GSTP1_C.-18G>A(Promoter) & AM_10430 & rs8191439 & Ch11:67351297 \\
\hline GSTP1 & N & GSTP1_c.21C>G(V7V) & AM_10434 & rs8191444 & Ch11:67351624 \\
\hline GSTP1 & D147Y & GSTP1_c.439G>T(D147Y) & AM_10444 & rs4986949 & Ch11:67353677 \\
\hline GSTT1 & $*_{B}$ & GSTT1*B_C.310A>C(T104P) & AM_12242 & rs11550605 & Ch22:24379402 \\
\hline GSTT1 & A21T & GSTT1_c.61G>A(A21T) & AM_12245 & rs2266635 & Ch22:24384171 \\
\hline GSTT1 & $\mathrm{F} 45 \mathrm{C}$ & GSTT1_C.134T>G(F45C) & AM_12243 & rs17856199 & Ch22:24381766 \\
\hline GSTT1 & N & GSTT1_c.354G>A(V118V) & AM_12241 & rs2266636 & Ch22:24376996 \\
\hline GSTT1 & V169l & GSTT1_c.505G>A(V169I) & AM_12239 & rs2266637 & Ch22:24376845 \\
\hline NAT1 & $\star 11$ & NAT1*11_C.-344C>T & AM_14965 & rs4986988 & Ch8:18079213 \\
\hline NAT1 & *11 & NAT1*11_C.-40A>T & AM_14966 & rs4986989 & Ch8:18079517 \\
\hline NAT1 & $\star 27$ & NAT1*27_c.21T >G(L7L) & AM_14968 & rs4986992 & Ch8:18079577 \\
\hline NAT1 & *19 & NAT1*19_C.97C >T(R33X) & AM_14969 & rs56318881 & Ch8:18079653 \\
\hline NAT1 & R64W & NAT1_C.190C>T(R64W) & AM_14970 & rs56379106 & Ch8:18079746 \\
\hline NAT1 & *5 & NAT1*5_C.350GG>CC(R117T) & AM_14971 & rs72554606 & Ch8:18079906 \\
\hline NAT1 & V149| & NAT1_c.445G>A(V149I) & AM_14974 & rs4987076 & Ch8:18080001 \\
\hline NAT1 & *11 & NAT1*11_c.459G>A(T153T) & AM_14975 & rs4986990 & Ch8:18080015 \\
\hline NAT1 & *5 & NAT1*5_c.497GGG>CCC(RE166TQ) & AM_14976 & rs72554608 & Ch8:18080053 \\
\hline NAT1 & *15 & NAT1*15_C.559C>T(R187X) & AM_14977 & rs5030839 & Ch8:18080115 \\
\hline NAT1 & *14 & NAT1*14_C.560G>A(R187Q) & AM_14978 & rs4986782 & Ch8:18080116 \\
\hline NAT1 & *11 & NAT1*11_c.640T>G(S214A) & AM_14981 & rs4986783 & Ch8:18080196 \\
\hline NAT1 & $* 22$ & NAT1*22_c.752A>T(D251V) & AM_14982 & rs56172717 & Ch8:18080308 \\
\hline NAT1 & N & NAT1_c.777T>C(S259S) & AM_14983 & rs4986991 & Ch8:18080333 \\
\hline NAT1 & $\star 5$ & NAT1*5_884A>G(3'UTR) & AM_14986 & rs55793712 & Ch8:18080440 \\
\hline NAT1 & $\star 5$ & NAT1*5_976delA(3'UTR) & AM_14988 & rs72554612 & Ch8:18080532 \\
\hline NAT1 & N & NAT1_C.-11982A>T & AM_14963 & rs72554605 & Ch8:18067575 \\
\hline NAT1 & N & NAT1_c.363C>T(V121V) & AM_14973 & rs8190858 & Ch8:18079919 \\
\hline NAT1 & T207I & NAT1_c.620C>T(T207I) & AM_14980 & rs4987195 & Ch8:18080176 \\
\hline NAT1 & N & NAT1_931C>T(3'UTR) & AM_14987 & rs4986994 & Ch8:18080487 \\
\hline NAT1 & N & NAT1_1191G>T(3'UTR) & AM_14993 & rs4986993 & Ch8:18080747 \\
\hline NAT2 & *19 & NAT2*19_C.190C $>T(R 64 W)$ & AM_14998 & rs1805158 & Ch8:18257703 \\
\hline
\end{tabular}




\begin{tabular}{|c|c|c|c|c|c|}
\hline \multirow{2}{*}{$\begin{array}{l}\text { Gene } \\
\text { NAT2 }\end{array}$} & \multicolumn{2}{|c|}{$\begin{array}{l}\text { Summary Common Name } \\
\text { Flag }\end{array}$} & \multirow{2}{*}{$\begin{array}{l}\text { Probe Set } \\
\text { ID } \\
\text { AM_14999 }\end{array}$} & \multirow{2}{*}{$\begin{array}{l}\text { dbSNP RS } \\
\text { ID } \\
\text { rs1801279 }\end{array}$} & \multirow{2}{*}{$\begin{array}{l}\text { Chrom } \\
\text { Ch8:18257704 }\end{array}$} \\
\hline & *14 & NAT2*14_c.191G>A(R64Q) & & & \\
\hline NAT2 & $\star 5$ & NAT2*5_C.341T>C(I114T) & AM_15001 & rs1801280 & Ch8:18257854 \\
\hline NAT2 & *12D & NAT2*12D_C.364G>A(D122N) & AM_15002 & rs4986996 & Ch8:18257877 \\
\hline NAT2 & $\star 17$ & NAT2*17_C.434A>C(Q145P) & AM_15005 & rs72554616 & Ch8:18257947 \\
\hline NAT2 & *10 & NAT2*10_c.499G>A(E167K) & AM_15007 & rs72554617 & Ch8:18258012 \\
\hline NAT2 & $*_{6}$ & NAT2*6_c.590G>A(R197Q) & AM_15008 & rs1799930 & Ch8:18258103 \\
\hline NAT2 & *18 & NAT2*18_C.845A>C(K282T) & AM_15011 & rs56054745 & Ch8:18258358 \\
\hline NAT2 & $\star 7$ & NAT2*7_c.857G>A(G286E) & AM_15012 & rs1799931 & Ch8:18258370 \\
\hline NAT2 & N & NAT2_c.111T>C(F37F) & AM_14997 & rs72554615 & Ch8:18257624 \\
\hline NAT2 & $\mathrm{N}$ & NAT2_c.282C>T(Y94Y) & AM_15000 & rs1041983 & Ch8:18257795 \\
\hline NAT2 & L137F & NAT2_C.411A>T(L137F) & AM_15004 & rs4986997 & Ch8:18257924 \\
\hline NAT2 & $\mathrm{N}$ & NAT2_C.481C>T(L161L) & AM_15006 & rs1799929 & Ch8:18257994 \\
\hline NAT2 & $\mathrm{N}$ & NAT2_c.759C>T(V253V) & AM_15009 & rs56011192 & Ch8:18258272 \\
\hline NAT2 & K268R & NAT2_c.803A>G(K268R) & AM_15010 & rs1208 & Ch8:18258316 \\
\hline PTGIS & *3 & PTGIS*3_C.354T>A(S118R) & AM_12133 & rs5622 & Ch20:48164401 \\
\hline PTGIS & N & PTGIS_C.1117C>A(R373R) & AM_12126 & rs5629 & Ch20:48129706 \\
\hline PTGIS & $\star_{4}$ & PTGIS*4_C.1135C>A(R379S) & AM_12125 & rs56195291 & Ch20:48129688 \\
\hline PTGIS & N & PTGIS_c.258G>T(V86V) & AM_12134 & rs5621 & Ch20:48164497 \\
\hline PTGIS & Q134H & PTGIS_c.402G>T(Q134H) & AM_12132 & rs6067121 & Ch20:48160961 \\
\hline PTGIS & E154A & PTGIS_C.461A>C(E154A) & AM_12131 & rs5623 & Ch20:48160902 \\
\hline PTGIS & F171L & PTGIS_C.511T>C(F171L) & AM_12130 & rs5624 & Ch20:48160852 \\
\hline PTGIS & $\mathrm{N}$ & PTGIS_C.591C>T(R197R) & AM_12129 & rs5625 & Ch20:48156189 \\
\hline PTGIS & R236C & PTGIS_C.706C>T(R236C) & AM_12128 & rs5626 & Ch20:48140744 \\
\hline PTGIS & N & PTGIS_c.723A>G(L241L) & AM_12127 & rs5627 & Ch20:48140727 \\
\hline PTGIS & N & PTGIS_c.1239C>T(D413D) & AM_12124 & rs5630 & Ch20:48127684 \\
\hline PTGIS & P500S & PTGIS_C.1498C>T(P500S) & AM_12123 & rs5584 & Ch20:48124462 \\
\hline SLC15A1 & $\mathrm{N}$ & SLC15A1_c.22-40G>C & AM_10664 & rs8187819 & Ch13:99378743 \\
\hline SLC15A1 & V21I & SLC15A1_c.61G>A(V21I) & AM_10663 & rs8187818 & Ch13:99378664 \\
\hline SLC15A1 & F28Y & SLC15A1_c.83T>A(F28Y) & AM_10662 & rs8187817 & Ch13:99378642 \\
\hline SLC15A1 & $\mathrm{N}$ & SLC15A1_c.258G>A(S86S) & AM_10661 & rs8187823 & Ch13:99376273 \\
\hline SLC15A1 & $\mathrm{N}$ & SLC15A1_C.330C>T(N110N) & AM_10660 & rs8187822 & Ch13:99376201 \\
\hline SLC15A1 & S117N & SLC15A1_C.350G>A(S117N) & AM_10659 & rs2297322 & Ch13:99376181 \\
\hline SLC15A1 & S117R & SLC15A1_C.351C>A(S117R) & AM_10658 & rs8187821 & Ch13:99376180 \\
\hline SLC15A1 & $\mathrm{N}$ & SLC15A1_C.1179C>T(N393N) & AM_10653 & rs8187836 & Ch13:99358478 \\
\hline SLC15A1 & G419A & SLC15A1_C.1256G>C(G419A) & AM_10652 & rs4646227 & Ch13:99358401 \\
\hline SLC15A1 & $441 \mathrm{FS}$ & SLC15A1_c.1321insT & AM_10651 & rs72547504 & Ch13:99356637 \\
\hline SLC15A1 & N & SLC15A1_C.1347T>C(A449A) & AM_10650 & rs1339067 & Ch13:99356612 \\
\hline SLC15A1 & V450। & SLC15A1_C.1348G>A(V450I) & AM_10649 & rs2274828 & Ch13:99356611 \\
\hline SLC15A1 & $\mathrm{T} 451 \mathrm{~N}$ & SLC15A1_C.1352C>A(T451N) & AM_10648 & rs8187838 & Ch13:99356607 \\
\hline SLC15A1 & R459C & SLC15A1_C.1375C>T(R459C) & AM_10647 & rs2274827 & Ch13:99356584 \\
\hline SLC15A1 & N & SLC15A1_C.1446A>G(E482E) & AM_10645 & rs8187828 & Ch13:99354754 \\
\hline SLC15A1 & N & SLC15A1_C.1527C>T(N509N) & AM_10644 & rs8187832 & Ch13:99340771 \\
\hline SLC15A1 & P537S & SLC15A1_C.1609C>T(P537S) & AM_10643 & rs8187830 & Ch13:99340576 \\
\hline SLC15A1 & P586L & SLC15A1_C.1757C>T(P586L) & AM_10642 & rs56120058 & Ch13:99339905 \\
\hline SLC15A1 & $\mathrm{N}$ & SLC15A1_c.1848G>A(S616S) & AM_10641 & rs8187840 & Ch13:99338531 \\
\hline
\end{tabular}




\begin{tabular}{|c|c|c|c|c|c|}
\hline \multirow{2}{*}{$\begin{array}{l}\text { Gene } \\
\text { SLC15A2 }\end{array}$} & \multicolumn{2}{|c|}{$\begin{array}{l}\text { SummaryCommon Name } \\
\text { Flag }\end{array}$} & \multirow{2}{*}{$\begin{array}{l}\text { Probe Set } \\
\text { ID } \\
\text { AM_13300 }\end{array}$} & \multirow{2}{*}{$\begin{array}{l}\text { dbSNP RS } \\
\text { ID } \\
\text { rs } 2257212\end{array}$} & \multirow{2}{*}{$\begin{array}{l}\text { Chrom } \\
\text { Ch3:121643804 }\end{array}$} \\
\hline & L350F & SLC15A2_C.1048C>T(L350F) & & & \\
\hline SLC15A2 & P4095 & SLC15A2_C.1225C>T(P409S) & AM_13306 & rs1143671 & Ch3:121647286 \\
\hline SLC15A2 & R509K & SLC15A2_C.1526G>A(R509K) & AM_13307 & rs1143672 & Ch3:121648168 \\
\hline SLC15A2 & N & SLC15A2_C.141C>T(A47A) & AM_13282 & rs1143669 & Ch3:121615288 \\
\hline SLC15A2 & $\mathrm{R} 57 \mathrm{H}$ & SLC15A2_C.170G>A(R57H) & AM_13283 & rs1920305 & Ch3:121615317 \\
\hline SLC15A2 & N & SLC15A2_c.852G>A(A284A) & AM_13298 & rs2293616 & Ch3:121641693 \\
\hline SLC15A2 & N & SLC15A2_C.1161A>G(A387A) & AM_13301 & rs1143670 & Ch3:121646641 \\
\hline SLC15A2 & M704L & SLC15A2_C.2110A>C(M704L) & AM_13315 & rs1920314 & Ch3:121659774 \\
\hline SLC15A2 & N & SLC15A2_C.*6C>T(3'UTR) & AM_13316 & rs1920313 & Ch3:121659860 \\
\hline SLC15A2 & N & SLC15A2_c.*146T>G(3'UTR) & AM_13318 & rs4388019 & Ch3:121660000 \\
\hline SLC22A1 & S14F & SLC22A1_C.41C>T(S14F) & AM_14342 & rs34447885 & Ch6:160543008 \\
\hline SLC22A1 & $19 F S$ & SLC22A1_c.54insC & AM_14343 & rs72552761 & Ch6:160543021 \\
\hline SLC22A1 & L23V & SLC22A1_C.67C $>G(L 23 V)$ & AM_14344 & rs34570655 & Ch6:160543034 \\
\hline SLC22A1 & G38D & SLC22A1_C.113G>A(G38D) & AM_14345 & rs35888596 & Ch6:160543080 \\
\hline SLC22A1 & N & SLC22A1_c.156T>C(S52S) & AM_14347 & rs1867351 & Ch6:160543123 \\
\hline SLC22A1 & $\mathrm{R} 61 \mathrm{C}$ & SLC22A1_C.181C>T(R61C) & & rs12208357 & Ch6:160543148 \\
\hline SLC22A1 & L85F & SLC22A1_c.253C>T(L85F) & & rs35546288 & Ch6:160543220 \\
\hline SLC22A1 & $\mathrm{C} 88 \mathrm{R}$ & SLC22A1_C.262T>C(C88R) & & rs55918055 & Ch6:160543229 \\
\hline & F160L & & & & \\
\hline SLC22A1 & S189L & SLC22A1_C.566C>T(S189L) & & & 60553314 \\
\hline SLC22A1 & G220V & SLC22A1_c.659G>T(G220V) & AM_14354 & rs36103319 & Ch6:160553407 \\
\hline SLC22A1 & P283L & SLC22A1_C.848C>T(P283L) & AM_14357 & & Ch6:160557260 \\
\hline SLC22A1 & R287G & SLC22A1_c.859C>G(R287G) & AM_14358 & rs4646278 & Ch6:160557271 \\
\hline SLC22A1 & P341L & SLC22A1_C.1022C>T(P341L) & AM_14359 & rs2282143 & Ch6:160557643 \\
\hline SLC22A1 & G401S & SLC22A1_c.1201G>A(G401S) & AM_14361 & rs34130495 & Ch6:160560824 \\
\hline SLC22A1 & N & SLC22A1_C.1209C>T(|403|) & AM_14362 & rs35373824 & Ch6:160560832 \\
\hline SLC22A1 & V408M & SLC22A1_c.1222G>A(V408M) & AM_14363 & rs628031 & Ch6:160560845 \\
\hline SLC22A1 & M420del & $\begin{array}{l}\text { SLC22A1_c.1260_1262delGAT } \\
\text { (M420del) }\end{array}$ & AM_14368 & rs72552763 & Ch6:160560883 \\
\hline SLC22A1 & $\mathrm{M} 440 \mathrm{I}$ & SLC22A1_c.1320G>A(M440I) & AM_14371 & rs35956182 & Ch6:160564616 \\
\hline SLC22A1 & V461। & SLC22A1_c.1381G>A(V461I) & AM_14372 & rs34295611 & Ch6:160564677 \\
\hline SLC22A1 & G465R & SLC22A1_c.1393G>A(G465R) & AM_14373 & rs34059508 & Ch6:160575837 \\
\hline SLC22A1 & $\mathrm{R} 488 \mathrm{M}$ & SLC22A1_C.1463G>T(R488M) & AM_14374 & rs35270274 & Ch6:160575907 \\
\hline SLC22A1 & N & SLC22A1_c.1647A>C(S549S) & AM_14376 & rs16891138 & Ch6:160579596 \\
\hline SLC22A1 & N & SLC22A1_C.*15G>A(3'UTR) & AM_14377 & rs9457846 & Ch6:160579629 \\
\hline SLC22A1 & N & SLC22A1_C.*23G>A(3'UTR) & AM_14379 & rs34108432 & Ch6:160579637 \\
\hline SLC22A2 & 45FS & SLC22A2_C.134insA & AM_14403 & rs72552765 & Ch6:160679656 \\
\hline SLC22A2 & P54S & SLC22A2_C.160C>T(P54S) & AM_14402 & rs8177504 & Ch6:160679630 \\
\hline SLC22A2 & N & SLC22A2_c.390G>T(T130T) & AM_14401 & rs624249 & Ch6:160679400 \\
\hline SLC22A2 & *5 & SLC22A2*5_C.481T >C(F161L) & AM_14400 & rs8177509 & Ch6:160677683 \\
\hline SLC22A2 & M165V & SLC22A2_c.493A>G(M165V) & AM_14399 & rs8177508 & Ch6:160677671 \\
\hline SLC22A2 & M165I & SLC22A2_C.495G>A(M165I) & AM_14398 & rs8177507 & Ch6:160677669 \\
\hline SLC22A2 & *8 & SLC22A2*8_c.669C>T(I223I) & AM_14396 & rs8177510 & Ch6:160671584 \\
\hline SLC22A2 & A270S & SLC22A2_c.808G>T(A270S) & AM_14395 & rs316019 & Ch6:160670282 \\
\hline SLC22A2 & A297G & SLC22A2_c.890C>G(A297G) & AM_14394 & rs8177513 & Ch6:160668283 \\
\hline
\end{tabular}




\begin{tabular}{|c|c|c|c|c|c|}
\hline \multirow{2}{*}{$\begin{array}{l}\text { Gene } \\
\text { SLC22A2 }\end{array}$} & \multicolumn{2}{|c|}{$\begin{array}{l}\text { SummaryCommon Name } \\
\text { Flag }\end{array}$} & \multirow{2}{*}{$\begin{array}{l}\text { Probe Set } \\
\text { ID } \\
\text { AM_14392 }\end{array}$} & \multirow{2}{*}{$\begin{array}{l}\text { dbSNP RS } \\
\text { ID } \\
\text { rs } 8177516\end{array}$} & \multirow{2}{*}{$\begin{array}{l}\text { Chrom } \\
\text { Ch6:160664685 }\end{array}$} \\
\hline & $\star 7$ & SLC22A2*7_C.1198C>T(R400C) & & & \\
\hline SLC22A2 & $N$ & SLC22A2_C.1203C>T(|401I) & AM_14391 & rs8177515 & Ch6:160664680 \\
\hline SLC22A2 & K432Q & SLC22A2_c.1294A>C(K432Q) & AM_14390 & rs8177517 & Ch6:160663420 \\
\hline SLC22A2 & N & SLC22A2_C.1398C>T(G466G) & AM_14388 & rs8177520 & Ch6:160662609 \\
\hline SLC22A2 & N & SLC22A2_C.1506A>G(V502V) & AM_14386 & rs316003 & Ch6:160645832 \\
\hline SLC22A2 & N & SLC22A2_C.1587C>T(A529A) & AM_14385 & rs8177521 & Ch6:160645751 \\
\hline SLC22A2 & R463K & SLC22A2_C.1388G>A(R463K) & AM_14389 & rs3907239 & Ch6:160663326 \\
\hline SLC22A6 & G14S & SLC22A6_C.40G>A(G14S) & AM_10348 & rs72559735 & Ch11:62752123 \\
\hline SLC22A6 & $\mathrm{R} 50 \mathrm{H}$ & SLC22A6_C.149G>A(R50H) & AM_10347 & rs11568626 & Ch11:62752014 \\
\hline SLC22A6 & 57FS & SLC22A6_C.169delC(L57X) & AM_10346 & rs67264610 & Ch11:62751994 \\
\hline SLC22A6 & $1226 \mathrm{~T}$ & SLC22A6_C.677T>C(I226T) & AM_10340 & rs11568623 & Ch11:62749434 \\
\hline SLC22A6 & A256V & SLC22A6_C.767C >T(A256V) & AM_10339 & rs11568624 & Ch11:62749344 \\
\hline & R293W & & & & Ch11:62748776 \\
\hline & R454Q & & & & \\
\hline & $113 \mathrm{~T}$ & & & & \\
\hline & N & & & & \\
\hline & N & & & & \\
\hline & N & & & & \\
\hline & N & & & & \\
\hline & N & & & & \\
\hline & & & & & \\
\hline SLCO1A2 & & SLCO1A2_c.404A>T(N135I) & & & \\
\hline SLCO1A2 & R168C & SLCO1A2_C.502C>T(R168C) & & & \\
\hline SLCO1A2 & E172D & & & & \\
\hline SLCO1A2 & & SLC01A2_c.830C>A(T277N) & & & Ch12:21453362 \\
\hline SLCO1A2 & $278 F S$ & SLCO1A2_c.833delA(N278X) & & & Ch12:21453359 \\
\hline SLCO1A2 & $1281 \mathrm{~V}$ & SLCO1A2_c.841A>G(I281V) & & & \\
\hline SLCO1A2 & & SLCO1A2_C.968T>C(L323P) & & & \\
\hline SLCO1A2 & I355V & SLC01A2_c.1063A>G(I355V) & AM_10524 & rs45628437 & Ch12:21450350 \\
\hline SLCO1A2 & T668S & SLC01A2_C.2003C>G(T668S) & AM_10518 & rs11568557 & Ch12:21422492 \\
\hline SLCO1B1 & *17 & SLC01B1*17_c.11187G>A(Promoter) & AM_10493 & rs4149015 & Ch12:21283322 \\
\hline SLCO1B1 & $\star_{2}$ & SLCO1B1*2_c.217T>C(F73L) & AM_10494 & rs56101265 & Ch12:21325716 \\
\hline SLCO1B1 & V82A & & AM_10495 & & \\
\hline SLCO1B1 & N130D & SLCO1B1*1B_C.388A>G(N130D) & AM_10496 & rs2306283 & Ch12:21329738 \\
\hline SLCO1B1 & $\star 16$ & SLCO1B1*16_c.452A>G(N151S) & AM_10497 & rs2306282 & Ch12:21329802 \\
\hline SLCO1B1 & $\star_{4}$ & SLCO1B1*4_C.463C>A(P155T) & AM_10498 & rs11045819 & Ch12:21329813 \\
\hline SLCO1B1 & *3 & SLCO1B1*3_c.467A>G(E156G) & AM_10499 & rs72559745 & Ch12:21329817 \\
\hline SLCO1B1 & $\star 5$ & SLCO1B1*5_C.521T>C(V174A) & AM_10500 & rs4149056 & Ch12:21331549 \\
\hline SLCO1B1 & *18 & SLCO1B1*18_c.578T>G(L193R) & AM_10502 & rs72559746 & Ch12:21331606 \\
\hline SLCO1B1 & $\star_{6}$ & SLC01B1*6_c.1058T>C(I353T) & AM_10509 & rs55901008 & Ch12:21353529 \\
\hline SLCO1B1 & $\star 7$ & SLC01B1*7_c.1294A>G(N432D) & AM_10512 & rs56387224 & Ch12:21355583 \\
\hline SLCO1B1 & $\star 8$ & SLC01B1*8_c.1385A>G(D462G) & AM_10513 & rs72559748 & Ch12:21358855 \\
\hline SLCO1B1 & *9 & SLC01B1*9_c.1463G>C(G488A) & AM_10514 & rs59502379 & Ch12:21358933 \\
\hline SLCO1B1 & *10 & SLC01B1*10_c.1964A>G(D655G) & AM_10516 & rs56199088 & Ch12:21392011 \\
\hline SLCO1B1 & *11 & SLCO1B1*11_c.2000A>G(E667G) & AM_10517 & rs55737008 & Ch12:21392047 \\
\hline
\end{tabular}




\begin{tabular}{|c|c|c|c|c|c|}
\hline \multirow{2}{*}{$\begin{array}{l}\text { Gene } \\
\text { SLCO1B1 }\end{array}$} & \multicolumn{2}{|c|}{$\begin{array}{l}\text { Summary Common Name } \\
\text { Flag }\end{array}$} & \multirow{2}{*}{$\begin{array}{l}\text { Probe Set } \\
\text { ID } \\
\text { AM_10501 }\end{array}$} & \multirow{2}{*}{$\begin{array}{l}\text { dbSNP RS } \\
\text { ID } \\
\text { rs4149057 }\end{array}$} & \multirow{2}{*}{$\begin{array}{l}\text { Chrom } \\
\text { Ch12:21331599 }\end{array}$} \\
\hline & $\mathrm{N}$ & SLC01B1_C.571T>C(L191L) & & & \\
\hline SLCO1B1 & $\mathrm{N}$ & SLC01B1_C.597C>T(F199F) & AM_10503 & rs2291075 & Ch12:21331625 \\
\hline SLCO1B1 & P336R & SLCO1B1_C.1007C>G(P336R) & AM_10508 & rs72559747 & Ch12:21353478 \\
\hline SLCO1B3 & A112S & SLC01B3_C.334G>T(A112S) & AM_10481 & rs4149117 & Ch12:21011480 \\
\hline SLCO1B3 & I233M & SLC01B3_c.699A>G(I233M) & AM_10482 & rs7311358 & Ch12:21015760 \\
\hline SLCO1B3 & $281 \mathrm{FS}$ & SLC01B3_c.841delA(K281X) & AM_10484 & N/A & Ch12:21028282 \\
\hline SLCO1B3 & $\mathrm{N}$ & SLC01B3_C.1272A>G(L424L) & AM_10485 & rs4149143 & Ch12:21032506 \\
\hline SLCO1B3 & $\mathrm{N}$ & SLC01B3_C.1557G>A(A519A) & AM_10487 & rs2053098 & Ch12:21036411 \\
\hline SLCO1B3 & N & SLC01B3_c.1833A>G(G611G) & AM_10490 & rs3764006 & Ch12:21054369 \\
\hline SLCO1B3 & $695 F S$ & SLC01B3_c.2083insC & AM_10492 & rs72559744 & Ch12:21069155 \\
\hline SLCO2B1 & *2 & SLCO2B1*2_C.1175C>T(T392I) & AM_10458 & rs1621378 & Ch11:74904362 \\
\hline SLCO2B1 & $\mathrm{N}$ & SLCO2B1_C.405G>A(P135P) & AM_10454 & rs1109407 & Ch11:74876951 \\
\hline SLCO2B1 & D215V & SLCO2B1_c.644A>T(D215V) & AM_10456 & rs72559740 & Ch11:74880413 \\
\hline SLCO2B1 & $\mathrm{N}$ & SLCO2B1_c.1434-138G>T & AM_10460 & rs2306167 & Ch11:74907421 \\
\hline SLCO2B1 & $\mathrm{N}$ & SLCO2B1_C.1512C>T(C504C) & AM_10462 & rs59305495 & Ch11:74907637 \\
\hline SULT1A1 & *2 & SULT1A1*2_c.638G>A(R213H) & AM_11005 & rs9282861 & Ch16:28617514 \\
\hline SULT1A1 & *3 & SULT1A1*3_c.667A>G(M223V) & AM_11004 & rs1801030 & Ch16:28617485 \\
\hline SULT1A2 & T7। & SULT1A2_c.20C>T(T7I) & AM_10997 & rs1136703 & Ch16:28607232 \\
\hline SULT1A2 & P19L & SULT1A2_C.56C>T(P19L) & AM_10996 & rs10797300 & Ch16:28607196 \\
\hline SULT1A2 & N235T & SULT1A2_c.704A>C(N235T) & AM_10989 & rs1059491 & Ch16:28603655 \\
\hline SULT1A2 & N239S & SULT1A2_c.716A>G(N239S) & AM_10988 & rs45472392 & Ch16:28603643 \\
\hline SULT1A3 & $\begin{array}{l}\text { P101L, } \\
\text { P101H }\end{array}$ & SULT1A3_C $>T>A(P 101$ LorH)_alte & eAM_11016 & rs67944833 & $\begin{array}{l}\text { Ch16:29473201, } \\
\text { Ch16:29473201 }\end{array}$ \\
\hline SULT1A3 & R144C & SULT1A3_C>T(R144C)_alternate & AM_11017 & rs67878449 & Ch16:29474723 \\
\hline SULT1A3 & K234N & SULT1A3_G>T(K234N)_alternate & AM_11018 & rs35044222 & Ch16:29475588 \\
\hline SULT1A3 & $\begin{array}{l}\text { P101L, } \\
\text { P101H }\end{array}$ & SULT1A3_C>T>A(P101LorH) & AM_11027 & rs67944833 & $\begin{array}{l}\text { Ch16:30212544, } \\
\text { Ch16:30212544 }\end{array}$ \\
\hline SULT1A3 & R144C & SULT1A3_C>T(R144C) & AM_11028 & rs67878449 & Ch16:30214068 \\
\hline SULT1A3 & K234N & SULT1A3_G>T(K234N) & AM_11029 & rs35044222 & Ch16:30214933 \\
\hline TBXAS1 & *2 & TBXAS1*2_c.182G>A(R61H) & AM_14917 & rs6138 & Ch7:139572123 \\
\hline TBXAS1 & *1B & TBXAS1*1B_c.360G>A(S120S) & AM_14918 & rs41275018 & Ch7:139636013 \\
\hline TBXAS1 & *3 & TBXAS1*3_c.483C>A(D161E) & AM_14920 & rs5768 & Ch7:139653196 \\
\hline TBXAS1 & *4 & TBXAS1*4_c.737A>G(N246S) & AM_14923 & rs55856189 & Ch7:139657478 \\
\hline TBXAS1 & *5 & TBXAS1*5_C.1069C>G(L357V) & AM_14928 & rs4529 & Ch7:139661964 \\
\hline TBXAS1 & $\star_{6}^{6}$ & TBXAS1*6_c.1249C>G(Q417E) & AM_14932 & rs4528 & Ch7:139715542 \\
\hline TBXAS1 & *7 & TBXAS1*7_c.1348G>A(E450K) & AM_14936 & rs8192868 & Ch7:139715641 \\
\hline TBXAS1 & $\star 8$ & TBXAS1*8_C.1352C>A(T451N) & AM_14937 & rs5763 & Ch7:139715645 \\
\hline TBXAS1 & *9 & TBXAS1*9_c.1397G>A(R466Q) & AM_14939 & rs41311778 & Ch7:139717500 \\
\hline TBXAS1 & *1D & TBXAS1*1D_C.*121G>A(3'UTR) & AM_14941 & rs56091454 & Ch7:139720020 \\
\hline TBXAS1 & V125I & TBXAS1_c.373G>A(V125I) & AM_14919 & rs8192833 & Ch7:139636026 \\
\hline TBXAS1 & L1631 & TBXAS1_c.487C>A(L163I) & AM_14921 & rs6137 & Ch7:139653200 \\
\hline TBXAS1 & N & TBXAS1_c.585C>G(A195A) & AM_14922 & rs1042561 & Ch7:139655300 \\
\hline TBXAS1 & K258E & TBXAS1_c.772A>G(K258E) & AM_14924 & rs5769 & Ch7:139657513 \\
\hline TBXAS1 & R261G & TBXAS1_c.781A>G(R261G) & AM_14925 & rs5770 & Ch7:139657522 \\
\hline TBXAS1 & Q317K & TBXAS1_c.949C>A(Q317K) & AM_14926 & rs5771 & Ch7:139661844 \\
\hline
\end{tabular}




\begin{tabular}{|c|c|c|c|c|c|}
\hline \multirow{2}{*}{$\begin{array}{l}\text { Gene } \\
\text { TBXAS1 }\end{array}$} & \multicolumn{2}{|c|}{$\begin{array}{l}\text { SummaryCommon Name } \\
\text { Flag }\end{array}$} & \multirow{2}{*}{$\begin{array}{l}\text { Probe Set } \\
\text { ID } \\
\text { AM_14927 }\end{array}$} & \multirow{2}{*}{$\begin{array}{l}\text { dbSNP RS } \\
\text { ID } \\
\text { rs } 6140\end{array}$} & \multirow{2}{*}{$\begin{array}{l}\text { Chrom } \\
\text { Ch7:139661890 }\end{array}$} \\
\hline & I332T & TBXAS1_C.995T>C(I332T) & & & \\
\hline TBXAS1 & E388K & TBXAS1_C.1162G>A(E388K) & AM_14929 & rs3735354 & Ch7:139706915 \\
\hline TBXAS1 & G390V & TBXAS1_C.1169G>T(G390V) & AM_14930 & rs5760 & Ch7:139706922 \\
\hline TBXAS1 & N & TBXAS1_c.1206G>A(L402L) & AM_14931 & rs5761 & Ch7:139706959 \\
\hline TBXAS1 & R425C & TBXAS1_C.1273C>T(R425C) & AM_14933 & rs5762 & Ch7:139715566 \\
\hline TBXAS1 & A430T & TBXAS1_C.1288G>A(A430T) & AM_14934 & rs4526 & Ch7:139715581 \\
\hline TBXAS1 & $N$ & TBXAS1_C.1305C>T(A435A) & AM_14935 & rs4527 & Ch7:139715598 \\
\hline TBXAS1 & $\mathrm{N}$ & TBXAS1_C.1384C>A(R462R) & AM_14938 & rs5764 & Ch7:139717487 \\
\hline TBXAS1 & R502Q & TBXAS1_c.1505G>A(R502Q) & AM_14940 & rs8192864 & Ch7:139717608 \\
\hline TPMT & *2 & TPMT*2_c.238G>C(A80P) & AM_13986 & rs1800462 & Ch6:18143955 \\
\hline TPMT & E98X & TPMT_c.292G>T(E98X) & AM_13985 & rs72552739 & Ch6:18143901 \\
\hline TPMT & $\star 3 B$ & TPMT*3B_c.460G>A(A154T) & AM_13980 & rs1800460 & Ch6:18139228 \\
\hline TPMT & $* 24$ & TPMT*24_c.537G>T(Q179H) & AM_13978 & rs6921269 & Ch6:18134078 \\
\hline TPMT & *4 & TPMT*4_c.626-1G>A(SpliceDefect) & AM_13977 & rs1800584 & Ch6:18131012 \\
\hline TPMT & *8 & TPMT*8_c.644G>A(R215H) & AM_13976 & rs56161402 & Ch6:18130993 \\
\hline TPMT & $\star 3 C$ & TPMT*3C_C.719A>G(Y240C) & AM_13973 & rs1142345 & Ch6:18130918 \\
\hline TPMT & N & TPMT_c.474C $>T(|158|)$ & AM_13979 & rs2842934 & Ch6:18139214 \\
\hline $\begin{array}{l}\text { UGT1A } \\
\text { COMMON }\end{array}$ & *76 & UGT1A1*76_C. *211C>T(3'UTR) & AM_13067 & rs10929303 & Ch2:234681416 \\
\hline $\begin{array}{l}\text { UGT1A } \\
\text { COMMON }\end{array}$ & $\star 78$ & UGT1A1*78_c. *339C>G(3'UTR) & AM_13068 & rs1042640 & Ch2:234681544 \\
\hline $\begin{array}{l}\text { UGT1A } \\
\text { COMMON }\end{array}$ & $\star 79$ & UGT1A1*79_c.*440C>G(3'UTR) & AM_13070 & rs8330 & Ch2:234681645 \\
\hline $\begin{array}{l}\text { UGT1A } \\
\text { COMMON }\end{array}$ & *11 & UGT1A1*11_c.923G>A(G308E) & AM_13043 & rs62625011 & Ch2:234675738 \\
\hline $\begin{array}{l}\text { UGT1A } \\
\text { COMMON }\end{array}$ & *9 & UGT1A1*9_c.992A>G(Q331R) & AM_13044 & rs72551348 & Ch2:234675807 \\
\hline $\begin{array}{l}\text { UGT1A } \\
\text { COMMON }\end{array}$ & *10 & UGT1A1*10_c.1021C>T(R341X) & AM_13045 & rs72551349 & Ch2:234676519 \\
\hline $\begin{array}{l}\text { UGT1A } \\
\text { COMMON }\end{array}$ & *4 & UGT1A1*4_c.1069C>T(Q357X) & AM_13046 & rs72551350 & Ch2:234676567 \\
\hline $\begin{array}{l}\text { UGT1A } \\
\text { COMMON }\end{array}$ & $\star 16$ & UGT1A1*16_c.1070A>G(Q357R) & AM_13047 & rs72551351 & Ch2:234676568 \\
\hline $\begin{array}{l}\text { UGT1A } \\
\text { COMMON }\end{array}$ & $\star 29$ & UGT1A1*29_C.1099C>G(R367G) & AM_13049 & rs55750087 & Ch2:234676880 \\
\hline $\begin{array}{l}\text { UGT1A } \\
\text { COMMON }\end{array}$ & $\star 20$ & UGT1A1*20_c.1102G>A(A368T) & AM_13050 & rs72551352 & Ch2:234676883 \\
\hline $\begin{array}{l}\text { UGT1A } \\
\text { COMMON }\end{array}$ & *3 & UGT1A1*3_C.1124C>T(S375F) & AM_13051 & rs72551353 & Ch2:234676905 \\
\hline $\begin{array}{l}\text { UGT1A } \\
\text { COMMON }\end{array}$ & $\star 17$ & UGT1A1*17_c.1143C>G(S381R) & AM_13052 & rs72551354 & Ch2:234676924 \\
\hline $\begin{array}{l}\text { UGT1A } \\
\text { COMMON }\end{array}$ & $\star 18$ & UGT1A1*18_C.1201G>C(A401P) & AM_13053 & rs72551355 & Ch2:234676982 \\
\hline $\begin{array}{l}\text { UGT1A } \\
\text { COMMON }\end{array}$ & *24 & UGT1A1*24_c.1309A>T(K437X) & AM_13057 & rs72551357 & Ch2:234680912 \\
\hline $\begin{array}{l}\text { UGT1A } \\
\text { COMMON }\end{array}$ & $\mathrm{N}$ & UGT1A1_C.1428C>T(P476P) & AM_13060 & rs28900406 & Ch2:234681031 \\
\hline
\end{tabular}




\begin{tabular}{|c|c|c|c|c|c|}
\hline \multirow{2}{*}{$\begin{array}{l}\text { Gene } \\
\text { UGT1A } \\
\text { COMMON }\end{array}$} & \multicolumn{2}{|c|}{$\begin{array}{l}\text { SummaryCommon Name } \\
\text { Flag }\end{array}$} & \multirow{2}{*}{$\begin{array}{l}\text { Probe Set } \\
\text { ID } \\
\text { AM_13063 }\end{array}$} & \multirow{2}{*}{$\begin{array}{l}\text { dbSNP RS } \\
\text { ID } \\
\text { rs72551361 }\end{array}$} & \multirow{2}{*}{$\begin{array}{l}\text { Chrom } \\
\text { Ch2:234681090 }\end{array}$} \\
\hline & *55 & UGT1A1*55_c.1487T>A(L496X) & & & \\
\hline $\begin{array}{l}\text { UGT1A } \\
\text { COMMON }\end{array}$ & A511P & UGT1A1_c.1531G>C(A511P) & AM_13064 & rs1042709 & Ch2:234681134 \\
\hline UGT1A1 & $*_{60}$ & UGT1A1*60_c.-3279T>G(Promoter) & AM_13018 & rs4124874 & Ch2:234665659 \\
\hline UGT1A1 & *93 & UGT1A1*93_c.-3156G>A(Promoter) & AM_13019 & rs10929302 & Ch2:234665782 \\
\hline UGT1A1 & $\star 28$ & UGT1A1*28_c.TATA-box(Promoter) & AM_13024 & rs8175347 & Ch2:234668881 \\
\hline UGT1A1 & *6 & UGT1A1*6_c.211G>A(G71R) & AM_13030 & rs4148323 & Ch2:234669144 \\
\hline UGT1A1 & $\star 27$ & UGT1A1*27_c.686C>A(P229Q) & AM_13037 & rs35350960 & Ch2:234669619 \\
\hline UGT1A1 & N & UGT1A1_C.-2950A>G & AM_13020 & rs111741722 & Ch2:234665983 \\
\hline UGT1A1 & $\star 112$ & UGT1A1*112_C.-1353A>C & AM_13021 & rs3755319 & Ch2:234667582 \\
\hline UGT1A1 & $\star 80$ & UGT1A1*80_C.-364C>T & AM_13022 & rs887829 & Ch2:234668570 \\
\hline UGT1A1 & *45 & UGT1A1*45_c.222C>A(Y74X) & AM_13031 & rs72551340 & Ch2:234669155 \\
\hline UGT1A1 & *62 & UGT1A1*62_c.247T>C(F83L) & AM_13032 & rs56059937 & Ch2:234669180 \\
\hline UGT1A1 & $\star 12$ & UGT1A1*12_C.524T>A(L175Q) & AM_13033 & rs72551341 & Ch2:234669457 \\
\hline UGT1A1 & *15 & UGT1A1*15_C.529T>C(C177R) & AM_13034 & rs72551342 & Ch2:234669462 \\
\hline UGT1A1 & *8 & UGT1A1*8_C.625C>T(R209W) & AM_13035 & rs72551343 & Ch2:234669558 \\
\hline UGT1A1 & *43 & UGT1A1*43_c.698T>G(L233R) & AM_13038 & rs72551344 & Ch2:234669631 \\
\hline UGT1A1 & *14 & UGT1A1*14_c.826G>C(G276R) & AM_13040 & rs72551345 & Ch2:234669759 \\
\hline UGT1A3 & *5 & UGT1A3*5_c.17A>G(Q6R) & AM_13004 & rs28898617 & Ch2:234637789 \\
\hline UGT1A3 & W11R & UGT1A3_C.31T>C(W11R) & AM_13005 & rs3821242 & Ch2:234637803 \\
\hline UGT1A3 & $*_{4}$ & UGT1A3*4_C.133C>T(R45W) & AM_13008 & rs45625338 & Ch2:234637905 \\
\hline UGT1A3 & $\mathrm{N}$ & UGT1A3_c.81G>A(E27E) & AM_13007 & rs6706232 & Ch2:234637853 \\
\hline UGT1A3 & $N$ & UGT1A3_c.477A>G(A159A) & AM_13011 & rs7574296 & Ch2:234638249 \\
\hline UGT1A3 & 177FS & UGT1A3_c.529delC(P177X) & AM_13012 & rs72551338 & Ch2:234638301 \\
\hline UGT1A4 & $*_{2}$ & UGT1A4*2_c.70C>A(P24T) & AM_12991 & rs6755571 & Ch2:234627536 \\
\hline UGT1A4 & 207FS & UGT1A4_c.621delC(F207X) & AM_12999 & rs68014726 & Ch2:234628087 \\
\hline UGT1A5 & T78A & UGT1A5_c.232A>G(T78A) & AM_12980 & rs72551334 & Ch2:234621869 \\
\hline UGT1A5 & $\mathrm{N}$ & UGT1A5_c.285T>C(G95G) & AM_12981 & rs17874942 & Ch2:234621922 \\
\hline UGT1A5 & $\mathrm{H} 142 \mathrm{~N}$ & UGT1A5_C.424C>A(H142N) & AM_12982 & rs3755320 & Ch2:234622061 \\
\hline UGT1A5 & 207FS & UGT1A5_c.621delC(F207X) & AM_12986 & rs72551335 & Ch2:234622258 \\
\hline UGT1A6 & S7A & UGT1A6_c.19T>G(S7A) & AM_12969 & rs6759892 & Ch2:234601669 \\
\hline UGT1A6 & $\mathrm{N}$ & UGT1A6_C.315A>G(L105L) & AM_12973 & rs1105880 & Ch2:234601965 \\
\hline UGT1A6 & T181A & UGT1A6_c.541A>G(T181A) & AM_12974 & rs2070959 & Ch2:234602191 \\
\hline UGT1A6 & N & UGT1A6_c.627G>T(V209V) & AM_12976 & rs17863783 & Ch2:234602277 \\
\hline UGT1A6 & N & UGT1A6_c.57A>T(A19A) & AM_12971 & rs1042707 & Ch2:234601707 \\
\hline UGT1A6 & S7OY & UGT1A6_c.209C>A(S70Y) & AM_12972 & rs1042708 & Ch2:234601859 \\
\hline UGT1A7 & *12 & UGT1A7*12_c.-57T>G(5'UTR) & AM_12956 & rs7586110 & Ch2:234590527 \\
\hline UGT1A7 & *5 & UGT1A7*5_c.343G>A(G115S) & AM_12961 & rs61261057 & Ch2:234590926 \\
\hline UGT1A8 & *3 & UGT1A8*3_C.830G>A(C277Y) & AM_12932 & rs17863762 & Ch2:234527183 \\
\hline UGT1A8 & L105M & UGT1A8_c.313T>A(L105M) & AM_12911 & rs1126788 & Ch2:234526666 \\
\hline UGT1A8 & N & UGT1A8_C.315G>A(L105L) & AM_12912 & rs1126790 & Ch2:234526668 \\
\hline UGT1A8 & F109L & UGT1A8_c.327T>A(F109L) & AM_12913 & rs1126792 & Ch2:234526680 \\
\hline UGT1A8 & L110M & UGT1A8_c.328C>A(L110M) & AM_12914 & rs1126793 & Ch2:234526681 \\
\hline UGT1A8 & N118D & UGT1A8_c.352A>G(N118D) & AM_12915 & rs1126798 & Ch2:234526705 \\
\hline
\end{tabular}




\begin{tabular}{|c|c|c|c|c|c|}
\hline \multirow{2}{*}{$\begin{array}{l}\text { Gene } \\
\text { UGT1A8 }\end{array}$} & \multicolumn{2}{|c|}{$\begin{array}{l}\text { SummaryCommon Name } \\
\text { Flag }\end{array}$} & \multirow{2}{*}{$\begin{array}{l}\text { Probe Set } \\
\text { ID } \\
\text { AM_12924 }\end{array}$} & \multirow{2}{*}{$\begin{array}{l}\text { dbSNP RS } \\
\text { ID } \\
\text { rs1126803 }\end{array}$} & \multirow{2}{*}{$\begin{array}{l}\text { Chrom } \\
\text { Ch2:234526987 }\end{array}$} \\
\hline & M212V & UGT1A8_c.634A>G(M212V) & & & \\
\hline UGT1A8 & E216D & UGT1A8_c.648A>C(E216D) & AM_12925 & rs1126804 & Ch2:234527001 \\
\hline UGT1A9 & $\star 1 b$ & UGT1A9*1b_c.-118insT & AM_12947 & rs3832043 & Ch2:234580463 \\
\hline UGT1A9 & *3 & UGT1A9*3_c.98T>C(M33T) & AM_12949 & rs72551330 & Ch2:234580678 \\
\hline UGT1A9 & *4 & UGT1A9*4_c.726T>G(Y242X) & AM_12951 & rs66915469 & Ch2:234581306 \\
\hline UGT1A9 & $\star 5$ & UGT1A9*5_c.766G>A(D256N) & AM_12953 & rs58597806 & Ch2:234581346 \\
\hline UGT1A9 & C $3 W$ & UGT1A9_C.9C>G(C3W) & AM_12948 & rs72551329 & Ch2:234580589 \\
\hline UGT1A10 & $\star 5$ & UGT1A10*5_c.177G>A(M59I) & AM_12938 & rs56935833 & Ch2:234545345 \\
\hline UGT1A10 & N & UGT1A10_C.597T>C(D199D) & AM_12941 & rs45523834 & Ch2:234545765 \\
\hline UGT1A10 & *6 & UGT1A10*6_c.605C>T(T202I) & AM_12942 & rs58704432 & Ch2:234545773 \\
\hline UGT2B7 & N & UGT2B7*2_C.-327G>A(Promoter) & AM_13458 & rs7662029 & Ch4:69961912 \\
\hline UGT2B7 & N & UGT2B7*2_C.-161C>T(Promoter) & AM_13459 & rs7668258 & Ch4:69962078 \\
\hline UGT2B7 & *3 & UGT2B7*3_c.211G>T(A71S) & AM_13460 & rs12233719 & Ch4:69962449 \\
\hline UGT2B7 & N & UGT2B7_c.735A>G(T245T) & AM_13463 & rs28365062 & Ch4:69964271 \\
\hline UGT2B7 & N & UGT2B7*2_c.801T>A(P267P) & AM_13464 & rs7438284 & Ch4:69964337 \\
\hline UGT2B7 & *2 & UGT2B7*2_c.802C>T(H268Y) & AM_13465 & rs7439366 & Ch4:69964338 \\
\hline UGT2B7 & N & UGT2B7_c.1062C>T(Y354Y) & AM_13468 & rs4348159 & Ch4:69972952 \\
\hline UGT2B15 & D85Y & UGT2B15_c.253G>T(D85Y) & AM_13439 & rs1902023 & Ch4:69536084 \\
\hline UGT2B15 & K523T & UGT2B15_C.1568A>C(K523T) & AM_13437 & rs4148269 & Ch4:69512847 \\
\hline UGT2B15 & K523T & UGT2B15_C.1568A>C(K523T) & AM_13444 & rs4148269 & Ch4:69512847 \\
\hline UGT2B15 & A500T & UGT2B15_c.1498G>A(A500T) & AM_13445 & rs72551390 & Ch4:69512917 \\
\hline UGT2B15 & $\mathrm{N}$ & UGT2B15_C.*131C>G(3'UTR) & AM_13443 & rs72551389 & Ch4:69512692 \\
\hline UGT2B15 & $\mathrm{N}$ & UGT2B15_C.*168C>T(3'UTR) & AM_13442 & rs3100 & Ch4:69512655 \\
\hline UGT2B15 & N & UGT2B15_C.*185A>T(3’UTR) & AM_13441 & rs4148271 & Ch4:69512637 \\
\hline UGT2B17 & N & UGT2B17_c.1006-2603T>C & AM_13428 & rs4860305 & Ch4:69420232 \\
\hline UGT2B17 & $\mathrm{N}$ & UGT2B17_c.1313+840A>G & AM_13425 & rs7436962 & Ch4:69415555 \\
\hline UGT2B17 & H450Y & UGT2B17_c.1348C>T(H450Y) & AM_13423 & rs72551385 & Ch4:69403588 \\
\hline VKORC1 & $\mathrm{N}$ & VKORC1_c.-1639G>A(Promoter) & AM_11054 & rs9923231 & Ch16:31107689 \\
\hline VKORC1 & $\mathrm{N}$ & VKORC1_c.173+324T>G & AM_11049 & rs2884737 & Ch16:31105554 \\
\hline VKORC1 & $\mathrm{N}$ & VKORC1_c.173+525C>T & AM_11047 & rs17708472 & Ch16:31105353 \\
\hline VKORC1 & $\mathrm{N}$ & VKORC1_C.174-136C>T & AM_11045 & rs9934438 & Ch16:31104878 \\
\hline VKORC1 & $\mathrm{N}$ & VKORC1_c.283+124G>C & AM_11043 & rs8050894 & Ch16:31104509 \\
\hline VKORC1 & N & VKORC1_c.283+837C>T & AM_11040 & rs2359612 & Ch16:31103796 \\
\hline VKORC1 & N & VKORC1_C.*134G>A(3'UTR) & AM_11034 & rs7294 & Ch16:31102321 \\
\hline VKORC1 & N & VKORC1_C.-5014T>C(Promoter) & AM_11061 & rs17884388 & Ch16:31111064 \\
\hline VKORC1 & N & VKORC1_C.-1877A>G(Promoter) & AM_11055 & rs17878544 & Ch16:31107927 \\
\hline VKORC1 & V29L & VKORC1_c.85G>T(V29L) & AM_11052 & rs104894539 & Ch16:31105966 \\
\hline VKORC1 & V45A & VKORC1_c.134T>C(V45A) & AM_11051 & rs104894540 & Ch16:31105917 \\
\hline VKORC1 & R58G & VKORC1_c.172A>G(R58G) & AM_11050 & rs104894541 & Ch16:31105879 \\
\hline VKORC1 & N & VKORC1_c.173+486C>A & AM_11048 & rs13337470 & Ch16:31105392 \\
\hline VKORC1 & $N$ & VKORC1_C.174-429C>T & AM_11046 & rs13336384 & Ch16:31105171 \\
\hline VKORC1 & V66M & VKORC1_c.196G>A(V66M) & AM_11044 & rs72547529 & Ch16:31104720 \\
\hline VKORC1 & N & VKORC1_c.283+186T>C & AM_11042 & rs17886199 & Ch16:31104447 \\
\hline VKORC1 & $N$ & VKORC1_c.283+231G>A & AM_11041 & rs17884850 & Ch16:31104402 \\
\hline VKORC1 & $\mathrm{N}$ & VKORC1_c.284-882A>T & AM_11039 & rs17884982 & Ch16:31103545 \\
\hline
\end{tabular}




\begin{tabular}{llllll}
\hline Gene & \multicolumn{2}{l}{$\begin{array}{l}\text { SummaryCommon Name } \\
\text { Flag }\end{array}$} & $\begin{array}{l}\text { Probe Set } \\
\text { ID }\end{array}$ & $\begin{array}{l}\text { dbSNP RS } \\
\text { ID }\end{array}$ & Chrom \\
\hline VKORC1 & R98W & VKORC1_C.292C>T(R98W) & AM_11038 & rs72547528 & Ch16:31102655 \\
VKORC1 & N & VKORC1_c.358C>T(L120L) & AM_11037 & rs7200749 & Ch16:31102589 \\
VKORC1 & L128R & VKORC1_c.383T>G(L128R) & AM_11036 & rs104894542 & Ch16:31102564 \\
VKORC1 & N & VKORC1_c.*131C>A(3'UTR) & AM_11035 & rs11540137 & Ch16:31102324 \\
\hline
\end{tabular}


4 


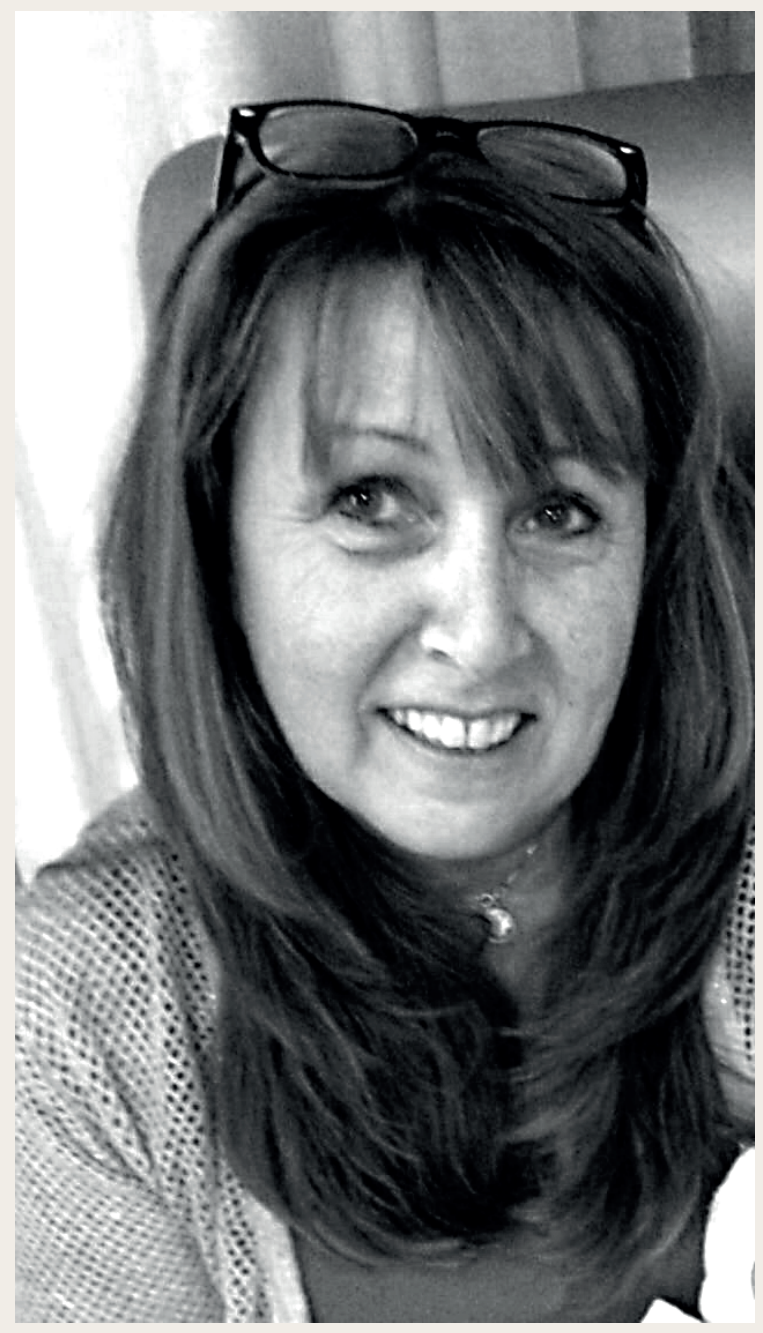


Chapter 05

Developing treatment for adrenocortical carcinoma

T.M.A. Kerkhofs, M.H.T. Ettaieb, I.G.C. Hermsen, H.R. Haak

Endocrine Related Cancer 2015 Dec;22(6): 325-38. 


\section{Abstract}

Cancer of the adrenal cortex (ACC) is a rare endocrine malignancy with limited treatment options. Patients typically present with autonomous hormonal overproduction and/ or a large abdominal mass. Hormonal assays and medical imaging can be diagnostic, but urinary steroid profiling might be a more sensitive technique to assess malignancy in adrenal tumours. Stage of disease at diagnosis is the most important prognostic factor. The current staging system needs refinement, especially to separate aggressive from indolent disease in stage IV patients and to select patients who need adjuvant treatment after complete surgical resection. Regarding the latter, assessing the proliferation index Ki-67 seems the best tool currently available. Genomic profiling is expected to become of clinical relevance in the future. Medical therapy is centred on the adrenolytic drug mitotane, which carries considerable toxicity and is not easy to manage. Its tolerability and long plasma level build-up phase may be improved by therapeutic drug monitoring based on pharmacokinetic modelling and intensive counselling of patients. Current chemotherapy regimens can offer disease stabilization in about $50 \%$ of patients, but an objective response should be expected in less than $25 \%$. Research on targeted therapy and immunotherapy is difficult in this rare disease with often heavily pre-treated patients and has not yet been successful. Quality of care should be ensured by treating patients in centres with established experience in multidisciplinary oncologic care, who adhere to prevailing guidelines and state-ofart in diagnostic and treatment concepts. International collaboration in fundamental research and clinical trials is the key to further elucidate the pathogenesis and to improve patient care. 


\section{Introduction}

Cancer of the adrenocortical cortex (ACC) occurs in about 1-2 persons per million population, which renders it a rare endocrine malignancy (Kebebew et al. 2006, Fassnacht and Allolio 2009). Complete surgical resection is the sole curative option. In about half of all patients this is impossible because of presentation with metastasized disease (Kerkhofs et al. 2013c). Moreover, recurrent disease occurs in approximately $60 \%$ of operated patients according to national database studies (Hermsen et al. 2012, Erdogan et al. 2013). Upon presentation, prognosis is mainly determined by stage of disease. Patients with localized disease have a 5-year survival of 60 to $80 \%$, in patients with locally advanced disease this rate drops to 35 to 50\%. In metastasized disease, prognosis varies from 13 to 28\%, but heterogeneity in this group precludes an adequate estimation (Fassnacht et al. 2009, Tran et al. 2013, Kerkhofs et al. 2013c). The cornerstone of medical treatment is mitotane, a 55-year-old drug that is derived from an agricultural insecticide (Lubitz et al. 1973). The aim of this review is to discuss changes we foresee in diagnostic and prognostic instruments, as well as suggestions for clinical management and possibilities for future treatment of ACC.

\section{Diagnostics}

ACC patients may present with symptoms of an abdominal mass, symptoms related to autonomous hormonal production by the tumour, or both. In rare cases, ACC is discovered incidentally during diagnostic tests or medical imaging for reasons unrelated to the adrenal glands. Figure 1 contains a flow-chart of the diagnostic workup of patients with suspect ACC. Data on the incidence of hormonal overproduction in ACC differ as some studies report clinical symptoms in symptomatic patients and others report biochemical results. Clinical symptoms caused by autonomous and excessive production of glucocorticoids alone occur in about 30\% of patients according to an international multicenter study (Berruti et al. 2014). Interestingly, studies based on biochemical results report a lower rate of isolated glucocorticoid overproduction: 14 to 20\% (Berruti et al. 2005, Abiven et al. 2006). The discrepancy may be explained by underestimation of combined glucocorticoid/androgen excess in the multicenter clinical study given the reported rates of $8.3 \%$ versus 23 to $36 \%$ in the biochemical studies mentioned before. After all, biochemical analysis is expected to have higher sensitivity for hormonal abnormalities compared to clinical examination only. Overproduction of androgens alone was reported in 5 to 20\% (Berruti et al. 2005, Abiven et al. 2006, Berruti et al. 2014). Overproduction of oestrogens, mineralocorticoids or other steroid combinations are rare, being diagnosed in $<5 \%$ of patients. In 23 to 31\% (after endocrine work-up) to 48\% (based on clinical evaluation) of all ACC patients, hormonal overproduction is not apparent upon presentation. These patients typically seek medical attention because of abdominal pain, fullness 
or a palpable mass. Non-specific symptoms related to malignancy such as weight loss, night sweats or fever may also be present (Berruti et al. 2014). Research suggests that despite absence of clinically apparent hormonal overproduction, these tumours do produce excess steroid precursors such as tetrahydro-11deoxycortisol (THS), pregnanediol (P2), pregnanetriol (P3) and etiocholanolone (E) (Arlt et al. 2011). Examination of urine samples using highly sensitive methods such as gas chromatography/mass spectrometry (urinary steroid profiling, USP) is able to detect small amounts of these substances. Retrospective studies suggest that THS excretion $>2.35 \mu \mathrm{mol} / 24 \mathrm{~h}$ could be diagnostic for the presence of ACC (Kerkhofs et al. 2015b). Also, USP can be used to distinguish ACC from adenomas with degenerative or regressive characteristics on imaging (Perna et al. 2014). We expect the diagnostic value of urinary steroid profiling will be confirmed in prospective trials and this technique will gain a place in the diagnostic armamentarium used in analysing patients with adrenal tumours. A prospective study aimed at assessing cost-effectiveness of urinary steroid profiling in the work-up of adrenal tumours is currently recruiting (Structured Evaluation of adRENal Tumors Discovered Incidentally - Prospectively Investigating the Testing Yield [SERENDIPITY-trial], NCT02324647). A secondary objective is to assess the frequency of ACC among patients with adrenal incidentaloma at baseline or during follow-up. It is tempting to speculate that urinary excretion of steroid precursors could be a marker for disease recurrence and/ or occurrence of metastases. Following this reasoning, it is not inconceivable that urinary samples could eliminate the need of frequent CT-scans and the associated exposure to ionizing radiation. Further research will have to determine whether USP has adequate sensitivity for recurrent disease in post-operative follow-up of patients with ACC. Another promising technique is measurement of circulating tumour cells in the bloodstream. Although difficult to detect in peripheral blood, these cells are considered to be an early marker of metastatic disease in several solid tumours and possibly a marker of therapeutic response. Thanks to technical advances, methods of detection are improving which results in increased sensitivity and specificity (Sun et al. 2011). In a recent preliminary study among 24 patients with adrenal tumours, circulating tumour cells were detected in all ACC patients, but not in patients with an adrenal adenoma (Pinzani et al. 2013). Additional larger studies are needed, since this technique seems potentially valuable as marker for detection of malignancy and perhaps even for disease progression or treatment monitoring.

\section{Clinical prognostic factors}

Currently, the most important prognostic factor in ACC is stage of disease at presentation (Fassnacht et al. 2009). The ENSAT staging system was introduced in 2009 and proved to be superior to the International Union Against Cancer (UICC) 
staging system in a 2010 validation study (Lughezzani et al. 2010). Additional prognostic factors are needed, since the ENSAT system does not result in a differentiation firm enough to base important treatment decisions on.

\section{Localized disease}

Overall survival was not significantly different between patients with ENSAT stage I and stage II disease in validation studies (Lughezzani et al. 2010). In this group, the main question is which patients should receive adjuvant treatment after surgery. The immunohistochemical proliferation marker Ki-67 (Mib1) is regarded as the most promising prognostic factor to be included in standard work-up of ACC patients (McNicol et al. 1997, Terzolo et al. 2001, Sasano et al. 2006, Morimoto et al. 2008). A retrospective study among 569 patients with disease stages I-III who underwent a complete resection including an independent validation cohort demonstrated that Ki-67 is associated with recurrent disease (hazard ratio [HR] 1.042 per 1\% increase) and overall survival (HR for death, 1.051) (Beuschlein et al. 2015). The authors suggest clinical application of the Ki-67 index based on expression in tumour tissue of $<10 \%$, $10-19 \%$, and $\geq 20 \%$. There are limitations to clinical application of the Ki-67 index that deserve further attention. Research shows that inter-observer variability in determining the index is high, even when performed by expert pathologists. Preliminary results suggest that automated software assessment of the Ki-67 index may result in more consistent results and better correlation with clinical outcome (Pucci et al. 2014). In addition, Ki-67 proliferation within a single tumour is characterized by a highly heterogeneous pattern. Fields with highly proliferative cells are often surrounded by areas of non-staining cells or even necrosis, which holds a significant risk of sampling error. Therefore, a uniform methodology for assessing the Ki-67 index in ACC needs to be established.

\section{Advanced disease}

A second shortcoming of the ENSAT staging system is considerable heterogeneity in the stage IV group. In some patients disease progresses aggressively and hardly responds to treatment. As a result, survival is only a few weeks or months (Kerkhofs et al. 2013c). In other patients, the disease follows a more indolent course and survival of up to several years has been observed (Hermsen et al. 2008). The search for clinical predictors of survival in these patients is still ongoing. Several parameters have been suggested, such as age, mitotic rate or presence of autonomous hormone production (Abiven et al. 2006, Assie et al. 2007). A recent retrospective study from the ENSAT ACC working group proposes a modification of the ENSAT staging system regarding patients with stage III-IV disease (Libe et al. 2015). In this proposal, stage IV is divided 
in three subgroups (A, B and C, Table 1). Patients with $T_{3-4} N_{1} M_{0}$ disease shift from stage III to stage IV-A. Also in IV-A are patients with $T_{1-4} N_{0-1} M_{1}$, as long as no more than two different organs are affected (including lymph node metastases). Patients with $T_{1}$. ${ }_{4} \mathrm{~N}_{0-1} \mathrm{M}_{1}$ and three affected organs form group IV-B, all other patients (i.e. $T_{1-4} \mathrm{~N}_{0-1} \mathrm{M}_{1^{\prime}}>3$ organs) form group IV-C. In addition, the prognostic significance of four parameters was validated in a large cohort of stage III-IV patients ( $n=349)$. Tumour grade (G) was proposed as a binomial parameter defined as Weiss $\leq 6$ and Ki-67 $<20 \%$ or Weiss $>6$ and/or Ki-67 >20\%). Resection status (R), also binomial, was defined as complete resection $\left(R_{0}\right)$ or other. Age $(A)$ below 50 had a favourable effect on overall survival. Functional symptoms (F) was defined as the presence of tumour- or hormone related symptoms at diagnosis. The exact role of these parameters in further stratification of patients with advanced ACC will have to be established in future studies. It is expected that the differentiation between stage IV-A, B and C will have consequences for treatment initiation, for example to start with mitotane monotherapy in metastatic disease with relatively favourable characteristics, but start immediate combination chemotherapy in metastasized disease with unfavourable features.

\section{Genetic prognostic factors}

In addition to establishing clinical prognostic factors, research is focused on the emerging possibilities of genomic characterization of tumours. Gene expression profiling on large cohorts of adrenal tumours identified among ACCs two distinct subgroups that differed in long-term overall survival, while distinguishing histological features could not be found (De Reynies et al. 2009, Giordano et al. 2009). In an effort to further characterize genomic alterations in ACC, a study combined exome sequencing, SNP arrays, DNA methylation analysis, mRNA expression arrays and miRNA sequencing (Assie et al. 2014). Two clusters were identified based on mRNA expression that were associated with clinical outcome, i.e. overall survival. Tumours from the cluster with poor outcome (named C1A) could subsequently be divided in three groups based on DNA methylation profile. In this particular example, DNA methylation refers to hypermethylation of Cytosine-Guanine dinucleotides $(\mathrm{CpG})$. A region of DNA with a high frequency of CpG sites is called a CpG island. If hypermethylation of CpG islands occurs in the promotor region of a specific gene (for example a tumour suppressor gene), this may cause their dysfunction. In accordance with observations in colon cancer, subsets of hypermethylated ACCs have been identified and labelled CpG island methylator phenotype (CIMP) (Barreau et al. 2013). Hence, the three subgroups in the C1A cluster are labelled CIMP-high, CIMP-low and non-CIMP. Tumours from the cluster with indolent course of disease (named C1B) could be further divided in two groups based on miRNA expression (Mi1 and Mi2). Earlier research also suggested circulating miRNA was associated with clinical outcome: high levels of miR-483-5p 
and low levels of miR-195 were correlated with shorter recurrence-free and overall survival (Chabre et al. 2013). Analysis of suspect driver genes and pathways revealed most alterations in the subgroup with poor clinical outcome, as well as a higher mutation rate. It appears that inter-individual differences are large, given that the most frequently altered gene (ZNRF3, related to the $\beta$-catenin pathway) was altered in $21 \%$ of cases (Assie et al. 2014). It is expected that further genomic characterization will lead to increased understanding of ACC pathogenesis. In the future, genomic characterization of ACC is expected to be of prognostic value and improve clinical management. For example, an unfavourable genomic profile might warrant aggressive adjuvant (chemo)therapy and close surveillance, whereas a favourable profile could justify more lenient follow-up. In addition we expect improved understanding of the pathogenesis will also yield therapeutic targets and thus therapeutic possibilities.

\section{Organization and quality of care}

Concentration of care in a limited number of specialized hospitals aimed at improving outcomes is an important concept in general healthcare, but particularly in oncological care, high risk surgical care and rare diseases. Regarding ACC, increasing evidence suggests that establishment of (inter)national collaborative networks of expert centres has a favourable effect on survival (Fassnacht et al. 2010, Lombardi et al. 2012, Kerkhofs et al. 2013b). The concept that rare diseases should be treated in a limited number of specialized hospitals seems intuitively logical. Interestingly, a better outcome of oncological treatment in specialized centres is not necessarily correlated with volume requirements (Ho et al. 2012). Similar observations were reported in high-risk surgical treatment (Finks et al. 2011). Moreover, common minimum volume requirements as instituted for other cancers are hardly feasible for ACC due to the low incidence. For example, the number of new patients with ACC per year in the Netherlands is about 20 (median 21, range 13-26 between 1993 and 2010). Although the number of surgical procedures for suspect adrenal malignancy will be higher, the grand total is expected to be too low to institute meaningful minimum volume requirements. Of course, numbers are higher in other (bigger) countries, but it is questionable whether geographical spread would allow for concentration of care on this scale. We expect quality criteria other than volume are of greater importance in rare diseases. It seems more important that centres adhere to current state-ofart treatment concepts, which in turn seems best feasible in a specialized centres with dedicated physicians. Experience with multidisciplinary oncologic surgery and preferably adrenal/endocrine surgery is a strong recommendation and maybe even a prerequisite. In addition, participation in international networks and clinical trials should be encouraged. By doing so, local specialists remain up-to-date and rapid adjustments can be made while respecting national and local regulations. Figure 2 
contains a flowchart depicting current treatment strategy in which multidisciplinary decision-making has a key role. The following paragraphs discuss the steps of this treatment algorithm and highlight several possibilities for future improvement. Table 2 should be regarded as supplement to Figure 1 and 2 and summarizes the considerations that in our view should be part of current state-of-art diagnostic and treatment concepts in ACC.

\section{Surgery: the primary treatment modality}

In patients who present with local or locally advanced disease, curative treatment is possible by performing complete surgical resection (Schulick and Brennan 1999, Grubbs et al. 2010). In practice, this is not as straightforward as it may seem. In the first place, completeness of resection can be difficult to determine due to the often close anatomical relationship between tumour capsule and adjacent organs such as liver or kidney. As a consequence, in some cases the largest possible margin consists of tumour capsule only, which may be only few cell layers thick. Secondly, the necessity of lymph node dissection (LND) and subsequent resection has not yet been established. It has been suggested in (few) retrospective studies that locoregional LND is associated with improved survival (Reibetanz et al. 2012, Tran et al. 2013, Erdogan et al. 2013). In contrast, a study from the SEER database could not confirm a survival benefit after LND (Saade et al. 2015). Results are difficult to interpret since it remains unknown whether presumed LNDs were performed intentionally and, the other way around, whether pathologists recognized and/or described lymph nodes that in fact were intentionally dissected. Also, the anatomical extent of LND for adrenal tumours is not standardized. Reibetanz et al. presented a proposal for the field of LND on a sound theoretical basis, but this has not yet been tested in clinical practice (Reibetanz et al. 2012). The only way to settle this debate is to perform a prospective randomized trial with standardized surgical technique, but this seems difficult in such a rare disease. A third subject of debate is the surgical approach, i.e. laparoscopy or laparotomy. A minimally invasive technique is supposed to offer short-term benefits such as lower morbidity and shorter hospital stay (Brix et al. 2010, Porpiglia et al. 2010, Lombardi et al. 2012, Donatini et al. 2014). Those in favour of laparotomy advocate this approach holds a lower risk of tumour rupture (Miller et al. 2010, Miller et al. 2012, Cooper et al. 2013, Mir et al. 2013, Toniato. 2013). It is expected that individual preferences and skills are highly influential on the outcome. A literature review of 23 retrospective studies addressing this question concluded that open surgery should remain the default option in ACC (Jurowich et al. 2013). However, in case of 'limited size tumours' $(<10 \mathrm{~cm})$ radical resection through a laparoscopic approach should be technically feasible if performed by an experienced surgeon. If there is evidence of invasive disease, laparotomy should be performed as extensive resection might be necessary. 
In selected patients presenting with metastasized disease, debulking surgery might yield a survival benefit (Kerkhofs et al. 2013b, Dy et al. 2015). Prospective studies on this topic are not yet available, and retrospective studies inevitably hold selection bias: chances are that patients who underwent debulking surgery were in better clinical condition compared to patients who were not operated on. Patients with metachronous oligometastases might benefit from local surgical resection or other local ablative measures. Evidence is limited and these interventions are probably best applicable to patients with otherwise favourable prognostic characteristics (Wood et al. 2003, Cazejust et al. 2010, Baudin et al. 2011). In recurrent (local) disease, surgery is the treatment of choice. Main predictors of outcome in this situation are time to first recurrence and resectability of the recurred tumour (Erdogan et al. 2013). Patients with a time to recurrence longer than one year and a completely resected recurrence have the best prognosis. In patients with early and/or not completely resectable recurrence, surgical treatment may be considered.

\section{Mitotane}

Drug therapy with mitotane has since 1959 been in use for treatment of patients with irresectable or metastasized ACC (Bergenstal et al. 1960, Lubitz et al. 1973, Baudin et al. 2011, Fassnacht et al. 2011). Mitotane is derived from the insecticide dichlorodiphenyltrichloroethane (DDT) and is administered orally in tablets in two to three daily doses. The drug exerts an anti-neoplastic effect on ACC tissue and in addition inhibits cortisol synthesis, which is beneficial in patients with Cushing's syndrome (Southren et al. 1966, Fukushima et al. 1971, Touitou et al. 1978, Ghataore et al. 2012). The antineoplastic effect is correlated with the plasma level of mitotane. A therapeutic response was observed in patients with plasma levels $>14 \mathrm{mg} / \mathrm{L}$, which is therefore considered as the lower limit of the therapeutic window (Haak et al. 1994, Baudin et al. 2001). Mitotane is well known for its toxicity, in particular for the gastrointestinal tract (nausea, diarrhoea, vomiting) and the nervous system (ataxia, amnesia, confusion) (Daffara et al. 2008). Toxicity of mitotane appears to be correlated with its plasma concentration, which is why a plasma level of $20 \mathrm{mg} / \mathrm{L}$ is considered the upper limit of the therapeutic window (Baudin et al. 2001). Patients (and their physicians) intuitively want to achieve therapeutic mitotane concentrations as early as possible in order to rapidly establish antiproliferative efficacy. A problematic aspect is that the drug's half-life is quite variable between patients but is in general extremely long (13-159 days) (Moolenaar et al. 1981). Therefore, it takes a very long time (on average 3 months) to reach the therapeutic level between 14 and 20 mg/L (Terzolo et al. 2000, Faggiano et al. 2006, Kerkhofs et al. 2013a). Especially in patients presenting with stage IV disease (+/- 50\% of patients), this long build-up phase delays effective treatment. Also, co-administration of chemotherapy might 
affect mitotane pharmacokinetics by inducing or inhibiting enzymes involved in drug metabolism (Kerkhofs et al. 2013a). Pharmacokinetic studies demonstrated that mitotane concentrations in fatty tissue are approximately 200-fold higher than in plasma and drug distribution appears to be far more important than drug elimination (von Slooten et al. 1982). Currently, research is focused at optimizing dosing schedules with help of therapeutic drug monitoring and pharmacogenetics (D'Avolio et al. 2013, Kerkhofs et al. 2015a). The aim is to shorten the plasma level build-up time and to minimize drug toxicity. For example, in patients presenting with stage IV disease not amenable to surgical resection, short-term achievement of adequate mitotane monotherapy may postpone the necessity of the often toxic and invalidating etoposide/doxorubicin/cisplatin (EDP) chemotherapy. On a side note, EDP chemotherapy may be more effective in combination with adequate mitotane levels due to an interaction at the drug resistance protein, which is an additional argument to optimize mitotane treatment first (Bates et al. 1991, Berruti et al. 1998). In addition to the established application of mitotane in patients with advanced disease, mitotane is increasingly being used as adjuvant treatment after complete resection. This is based on convincing retrospective evidence suggesting prolonged recurrence-free and overall survival (Terzolo et al. 2007). According to the 2012 ESMO guidelines, adjuvant mitotane is recommended in patients with a high risk of disease recurrence defined by stage III disease, Ki-67>10\%, or incomplete resection (Berruti et al. 2012). Adjuvant treatment with mitotane is prospectively compared in a randomized trial that is currently recruiting patients with stage I-III disease who underwent a complete resection and had a Ki-67 <10\% (Efficacy of Adjuvant Mitotane Treatment in Prolonging Recurrence-free Survival in Patients With Adrenocortical Carcinoma at Low-intermediate Risk of Recurrence [ADIUVO-trial], NCT00777244). In this era of targeted therapy and drug engineering, it may seem unwise to rely on an old and toxic drug. However, no 'modern' drug has yet proven to be effective and possible new agents will have to be compared to mitotane first. Since mitotane is currently the only effective drug available, it will remain the backbone of medical therapy in ACC during the following years. Figure 3 contains a comprehensive flowchart of mitotane clinical management based on available literature and personal experience. It is important to realize that despite optimization of dosing schedules, the key factor influencing build-up of mitotane plasma levels is patient tolerability. From this perspective, the importance of adequate supportive treatment cannot be stressed enough, i.e. administration of hydrocortisone, anti-emetic and anti-diarrheal drugs if necessary. The strong CYP3A4 induction by mitotane necessitates careful selection of supportive drugs (van Erp et al. 2011, Kroiss et al. 2011). A possible strategy is to start concomitant treatment on day 1 including hydrocortisone $20 \mathrm{mg}$, metoclopramide $10 \mathrm{mg}$ two times daily (as needed) and loperamide $2 \mathrm{mg}$ two to four times daily (as needed). Hydrocortisone should be administered in supraphysiological 
doses because of CYP3A4 induction, which increases glucocorticoid metabolism (Chortis et al. 2013). Frequent assessment (every 3 months) of thyroid hormone status is necessary as mitotane may induce a clinical picture similar to central hypothyroidism, possibly through a direct effect on the pituitary gland or induction of thyroid hormone metabolism (Zatelli et al. 2010). Based on clinical experience, treatment with thyroxine supplementation restores euthyroidism. In men with signs of hypogonadism, assessment of testosterone and sex hormone binding globulin levels is warranted, as (yet unexplained) disturbances in mitotane treated patients are common (Berruti et al. 2012). Since mitotane inhibits 5-alpha-reductase, the enzyme that converts testosterone in the more potent metabolite 5a-dihydrotestosterone, treatment with testosterone supplementation may be ineffective. Supplementation of synthetic dihydrotestosterone should have the desired effect. Another (presumed) consequence of 5-alpha inhibition is increased conversion of testosterone to $17 \beta$-estradiol, which could explain the occurrence of gynecomastia in mitotanetreated men (Daffara et al. 2008, Chortis et al. 2013). Psychological and social aspects of treatment should not be neglected, i.e. professional counselling may be warranted. Follow-up on patient's well-being may be performed by questionnaire-based assessment of toxicity upon start of treatment and by repeating this assessment every 3 months. This facilitates early recognition and optimization of supportive treatment, and if necessary mitotane dose adjustments. In our experience educating patients about side-effects, taking time to register side-effects and providing instruments to alleviate them improves compliance and patient motivation to stay on treatment.

\section{Chemotherapy}

One of the greatest achievements in ACC research was the completion of the FIRMACT trial, which established the regimen EDP combined with mitotane as first-line chemotherapy in advanced ACC. An objective response was achieved in $23 \%$ of patients (Fassnacht et al. 2012). The secondary endpoint was defined as progression free survival at 8 weeks, which was achieved in 58\% of patients. The question could be asked whether a three-drug regimen is necessary to achieve these results. Therefore, a non-inferiority trial seems justified. On the other hand, given the overall poor prognosis in this group, it may be wiser to deploy available resources to a trial comparing new drugs to the existing regimen of EDP. Problematic is that no other regimens of cytotoxic drugs demonstrated results that even approximate those of EDP. Based on results of a phase II trial, a combined regimen of gemcitabine/ capecitabine is now offered as second-line therapy in Dutch and German expert centres. This regimen resulted in disease control during 6 months in 29\% of 28 patients (Sperone et al. 2010). Other studies including cytotoxic chemotherapy only produced disappointing results. Among the tested agents were capecitabine 
(combined with bevacizumab) (Wortmann et al. 2010), paclitaxel (Berruti et al. 2011), irinotecan (Baudin et al. 2002) and docetaxel (combined with cisplatin) (Urup et al. 2013). Again, mitotane is a problem in investigating new drugs since virtually all patients in advanced stages are pre-treated.

\section{Radiotherapy}

The application of adjuvant radiotherapy in ACC has so far only been studied in retrospective setting (Fassnacht et al. 2006, Sabolch et al. 2011, Habra et al. 2013). None of these studies demonstrated a benefit in disease-free or overall survival after adjuvant radiotherapy, whereas two studies did report a beneficial effect on the local recurrence rate (Fassnacht et al. 2006, Sabolch et al. 2011). Since local recurrence does not seem to be the most important predictor of survival, it is expected that systemic adjuvant therapy is more important than local control. In a different setting, i.e. after incomplete surgical resection, radiotherapy in combination with mitotane is recommended (Berruti et al. 2012). The rationale behind this recommendation is that patients with residual disease after surgery perform worse compared to patients who had a complete resection and aggressive treatment is warranted (Libe et al. 2015). Polat et al. suggested a dosing regimen based on clinical experience consisting of a total dose $>40$ Gy with single fractions of 1.8 Gy to $2 \mathrm{~Gy}$. This includes a boost volume to reach 50 Gy to 60 Gy in individual patients (Polat et al. 2009). As discussed above, there is a subgroup of ACC patients with indolent disease, for example with slowly progressive oligometastases in $\leq 2$ organs. Based on personal clinical experience (unpublished), we believe these patients might benefit from stereotactic body radiation therapy. This technique is continuously being refined and deserves further study as it holds the potential of prolonging local control in patients with often limited tumour load, in whom toxic systemic therapy would otherwise be the only option (Salama and Milano 2014). Another new technique in patients with metastasized disease is treatment with radioactive isotopes. A treatment protocol based on the radionuclide [131/]iodometomidate (IMTO) has been tested on 11 patients and resulted in a partial response in one patient and stable disease in five patients (Hahner et al. 2011). Further research will have to prove the potential of this promising treatment modality. Radiotherapy with palliative intent, for example irradiation of painful bone metastases or a large irresectable primary tumour, is widely accepted as a considerably effective measure. Evidence is again retrospective and mostly based on single-institution experience (Polat et al. 2009, Hermsen et al. 2010, Ho et al. 2013). 


\section{Targeted therapy}

Several targeted therapies have been tested in mostly small-scale trials; none of these has yet earned a place in the treatment algorithm. Among the targets examined in case series were the platelet derived growth factor receptor (PDGFR-R) and stem cell ligand receptor (Gross et al. 2006); epidermal growth factor receptor (EGFR)(Quinkler et al. 2008); vascular endothelial growth factor (VEGF)(Wortmann et al. 2010) and the mammalian target of rapamycin (mTOR) pathway (Fraenkel et al. 2013). An expanded phase I study combined the IGF-1R inhibitor cixutumumab and the mTOR inhibitor temsirolimus in 26 patients. Two phase II trials examined the efficacy of multi-tyrosine kinase inhibitors sorafenib and sunitinib in 10 and 38 patients respectively (Berruti et al. 2011, Kroiss et al. 2012). In general, these studies demonstrated disappointing results without any objective response and with only few patients experiencing a short-lived stabilization of their disease. So far, the only phase III trial involving a targeted treatment is a study on the IGF-1R inhibitor linsitinib (Fassnacht et al. 2015). A confirmed partial response was observed in three patients, but in the total population there was no increase in overall or progression-free survival. Interpretation of results from trials with targeted therapies is difficult for several reasons. In the first place, most patients who participated in these studies were pre-treated with mitotane and chemotherapy and were in advanced stage of their disease. It is likely that these tumours became drug-resistant and were hardly responsive to any therapy, or not responsive at all. Secondly, pre-treatment with mitotane and consequently CYP3A4 induction might have impaired the effectiveness of the therapies studied. This is a problematic aspect of mitotane treatment, especially since the half-life is so long and mitotane remains detectable in plasma months after cessation of treatment. It is unknown how long it takes for the inductive effect to wear off. As a result, timing of targeted therapies in clinical management (or trial participation for that matter) is difficult to determine. It seems logical to test potential new drugs in a setting where they can be compared to mitotane. Patients with advanced ACC (modified ENSAT stage IV-A) who had no prior systemic therapy and who would qualify for treatment with mitotane monotherapy (possibly after debulking surgery) could form the most suitable subgroup for such a trial. Thirdly, recent research made it increasingly clear that there is a subgroup of patients with metastatic disease and an indolent course of disease (Assie et al. 2014). It is not inconceivable that their natural course of disease resembles stable disease for several months while on a study drug. In future studies patients should be meticulously stratified, not only based on tumour characteristics but also based on disease progression in months or even years before participation. Research on immunotherapy in adrenocortical carcinoma is still at a preliminary stage. Blocking the Programmed Death-1 (PD-1) or Programmed Death-Ligand 1 (PDL1) receptor in an attempt to direct a T-cell response to tumour tissue has been successful in renal cell carcinoma, melanoma and non-small cell lung carcinoma 
(Topalian et al. 2012). Exploratory research on a small scale $(n=28)$ demonstrated ACC may express PD-L1 on the cell surface (Fay et al. 2015). A phase I study with the PD-L1 inhibitor Avelumab is currently recruiting and is open to ACC patients (NCT01772004). Other data demonstrated high expression of the survivin protein in a cohort of 29 ACCs, associated with a trend towards poor prognosis (Sbiera et al. 2013). These findings suggest survivin might be a target for immunotherapy, but this has not yet lead to a clinical trial.

\section{Research}

As discussed, there are many clinical questions yet unanswered and many trials to be performed. The European Network for the Study of Adrenal Tumours (ENSAT) is an expanding collaboration formed by researchers and clinicians. It holds a database with clinical data of >2200 ACC patients (as of June 2015), which is of great potential for future studies. Collection of biomaterial such as blood, urine and tumour samples is important in order to facilitate research. Due to high costs associated with especially genomic research projects, upscaling to European or even global platforms seems necessary to be able to fund these undertakings. National laws on the use of archival tumour tissue may differ. It is recommended to consult the local ethics committee on prevailing regulations regarding the establishment of a local biobank. A starting point should be to obtain informed consent from the patient for future (genomic) research on tumour tissue before surgery, even if the exact content of said research is not specified. International networks could facilitate this by supplying standard consent forms, issue standard operating procedures for tissue handling or even sponsor a central storage facility. In addition, international networks should promote development of international standards for reporting surgery and pathology results, determination of the Ki-67 index and urinary steroid profiling cut-off values.

\section{Conclusion}

The present review discusses current and future ACC diagnostics, prognostics and treatment concepts. In addition, it provides a view on organization of care and international collaboration. In the near future, we expect urinary steroid profiling to gain a place in daily practice diagnostics of adrenal tumours. Application in followup of ACC patients is promising but needs additional research. A recent proposal for refinement of the most important clinical prognostic factor, stage of disease, is expected to become standard practice. Currently, the best indicator of recurrencefree and overall survival is determination of the proliferation marker Ki-67. This technique needs standardization, since it will take several years before its successor, 
genomic profiling, will be ready for clinical practice. Risk stratification is needed to select patients for adjuvant treatment, since complete surgical resection alone, with or without lymph node dissection, cannot guarantee recurrence-free survival. Mitotane will remain the backbone of drug therapy in years to come. Its tolerability and long plasma level build-up phase may be improved by therapeutic drug monitoring based on pharmacokinetic modelling and intensive counselling of patients. Due to the rarity of disease, it is questionable whether expert centres should be instituted based on volume requirements. We advocate treatment of patients in centres with established experience in multidisciplinary oncologic care, who adhere to prevailing guidelines and state-of-art in diagnostic and treatment concepts. International collaboration is necessary to facilitate fundamental research and clinical trials to test new treatment possibilities that are so badly needed.

\section{Declaration of interest}

The authors declare that there is no conflict of interest that could be perceived as prejudicing the impartiality of the research reported.

\section{Funding}

This research did not receive any grant from any funding agency in the public, commercial or not-for-profit sector.

Table 1: Proposal for modified ENSAT staging system (Libé et al. 2014).

\begin{tabular}{ll}
\hline Stage & Definition \\
\hline III & $T_{3-4} N_{0} M_{0}$ \\
IV-A & $T_{1-4} N_{1} M_{0}$ or $T_{1-4} N_{0-1} M_{1}$ (max. 2 organs including $N$ ) \\
IV-B & $T_{1-4} N_{0-1} M_{1}$ (3 organs) \\
IV-C & $T_{1-4} N_{0-1} M_{1}$ (>3 organs) \\
\hline
\end{tabular}


Table 2: Summary of considerations that should be part of current state-of-art diagnostic and treatment concepts in adrenocortical carcinoma.

\begin{tabular}{ll}
\hline Step in algorithm & Consideration \\
\hline $\begin{array}{l}\text { Diagnostics in patients with suspect ACC (Figure 1) } \\
\text { Hormonal work-up }\end{array}$ & $\begin{array}{l}\text { Urinary steroid profiling can be of added value in patients with } \\
\text { inconclusive imaging results. }\end{array}$ \\
$\begin{array}{l}\text { Pre-operative } \\
\text { multidisciplinary }\end{array}$ & Takes place within 2 weeks after completion of diagnostic tests. \\
meeting & Attended by endocrinologist, oncologist, surgeon and radiologist.
\end{tabular}

\section{Treatment algorithm (Figure 2)}

Surgery

Post-operative multidisciplinary meeting

Surveillance

Consider ADIUVO

Adjuvant mitotane

Radiotherapy

Patient-tailored decision in metastatic disease
Adrenalectomy within 3 weeks after multidisciplinary meeting.

Resection status is determined by discussing surgeon's and pathologist's observations and conclusions.

Pathology report contains itemized Weiss-score and Ki-67 index.

Lymph node involvement is determined.

Definitive ENSAT-stage is determined.

FDG-PET scan performed 3-6 months after (complete) resection of primary tumour.

Possibility to enrol patients in (international) trials

Treatment instituted according to protocol (Figure 3) and maintained for at least 2 years.

Consider total dose $>40$ Gy with single fractions of 1.8 Gy to 2 Gy (including boost volume to reach 50 Gy to 60 Gy in individual patients) (Polat et al. 2009).

Consider cut-off values for number of affected organs from least to worst risk: 2, 3 or $>3$ (Libé et al. 2015).

Weiss-score $>6$ and/or Ki-67 >20\% correlates to most aggressive behaviour (Libé et al. 2015).

Consider performance status ECOG 2 or better as baseline before EDP-M treatment.

Treatment goal: take into account patient's wishes and expectations.

Mitotane monotherapy Treatment instituted according to protocol (Figure 3).

Local ablative measures Consider radio-frequency ablation, stereotactic body radiation therapy or surgical resection of oligometastases.

EDP+mitotane Treatment instituted according to FIRM-ACT protocol (Fassnacht et al. 2012)

\section{Optional: research facilitation}

Obtain informed consent to store and share clinical data for future research.

Obtain informed consent to store and share tumour tissue, blood sample, urine sample for future research.

Store freshly frozen sample of tumour tissue. 


\section{Figure legends:}

\section{Figure 1: Diagnostic work up of patients with suspect adrenocortical carcinoma.}

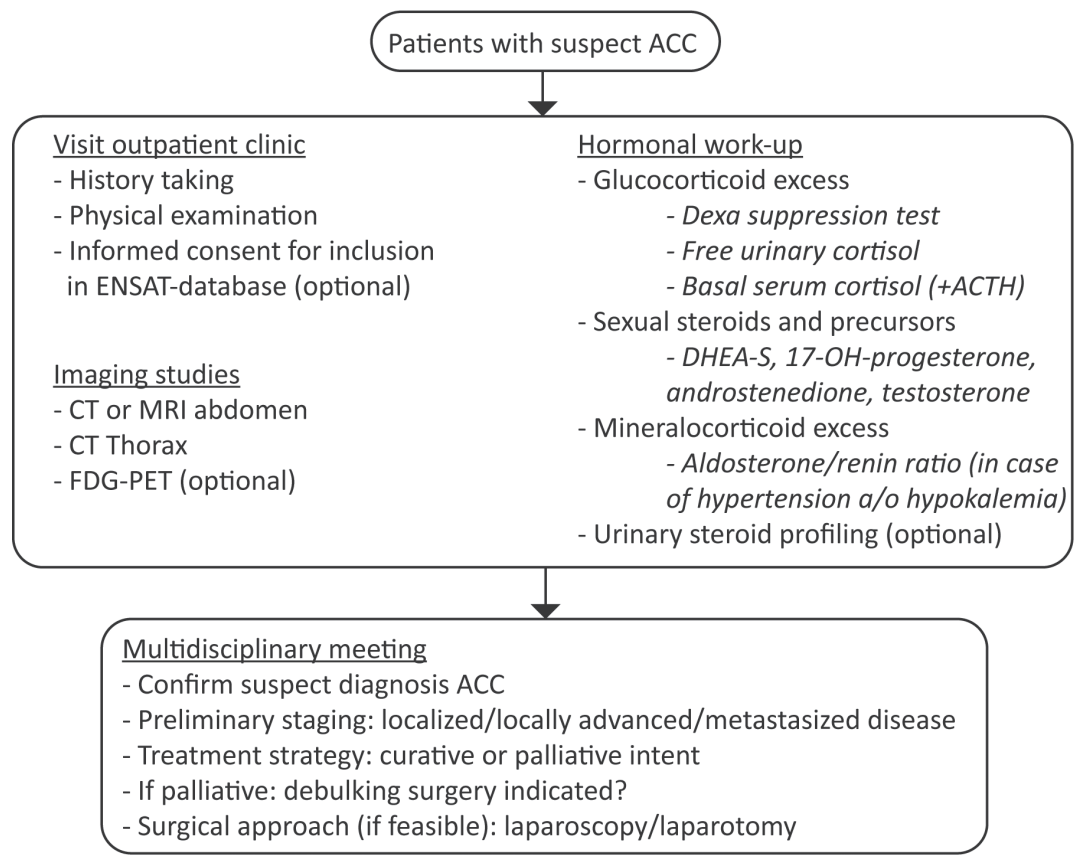




\section{Figure 2: Treatment algorithm for patients with adrenocortical carcinoma.}

R0: complete resection, R1: microscopic residual tumour, R2: macroscopic residual tumour, Rx: resection status undetermined, EDP: Etoposide/Doxorubicin/Cisplatin, ADIUVO: Clinical trial testing efficacy of Adjuvant Mitotane Treatment in Prolonging Recurrence-free Survival in Patients With Adrenocortical Carcinoma at Lowintermediate Risk of Recurrence.

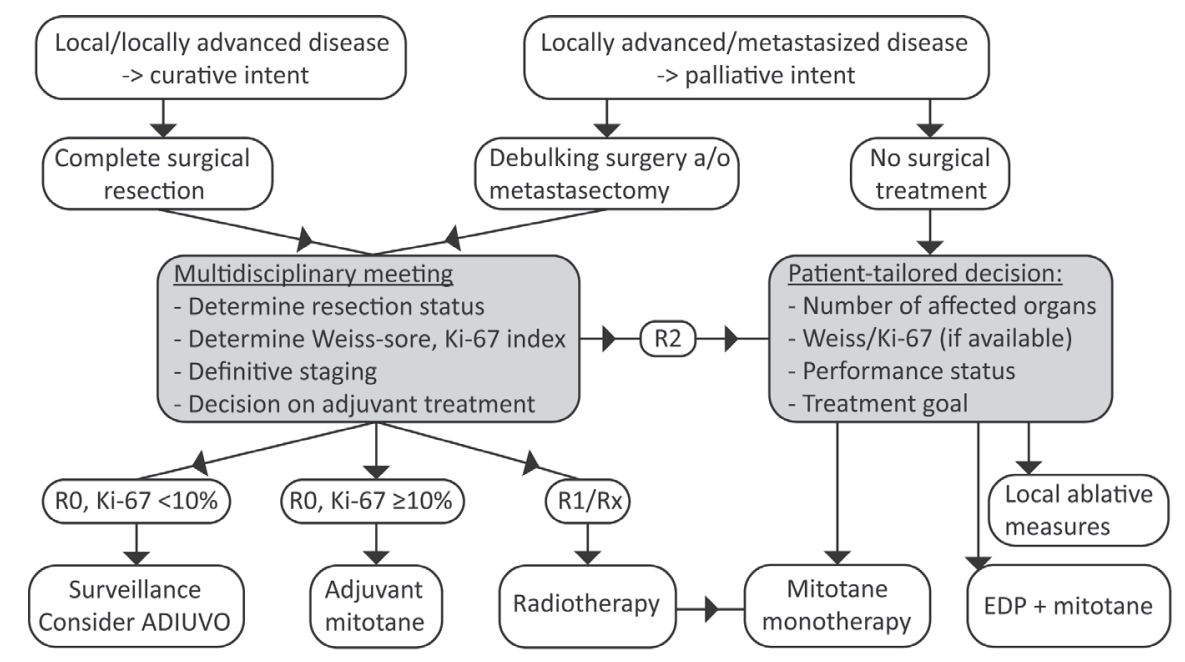




\section{Figure 3: Flowchart of clinical management in starting and maintaining mitotane treatment.}

CNS: central nervous system, Gl: gastro-intestinal, grading of side effects according to National Cancer Institute Common Terminology Criteria for Adverse Events ( $\mathrm{NCl}$ CTCAE) version 4, GGT: gamma-glutamyltransferase.

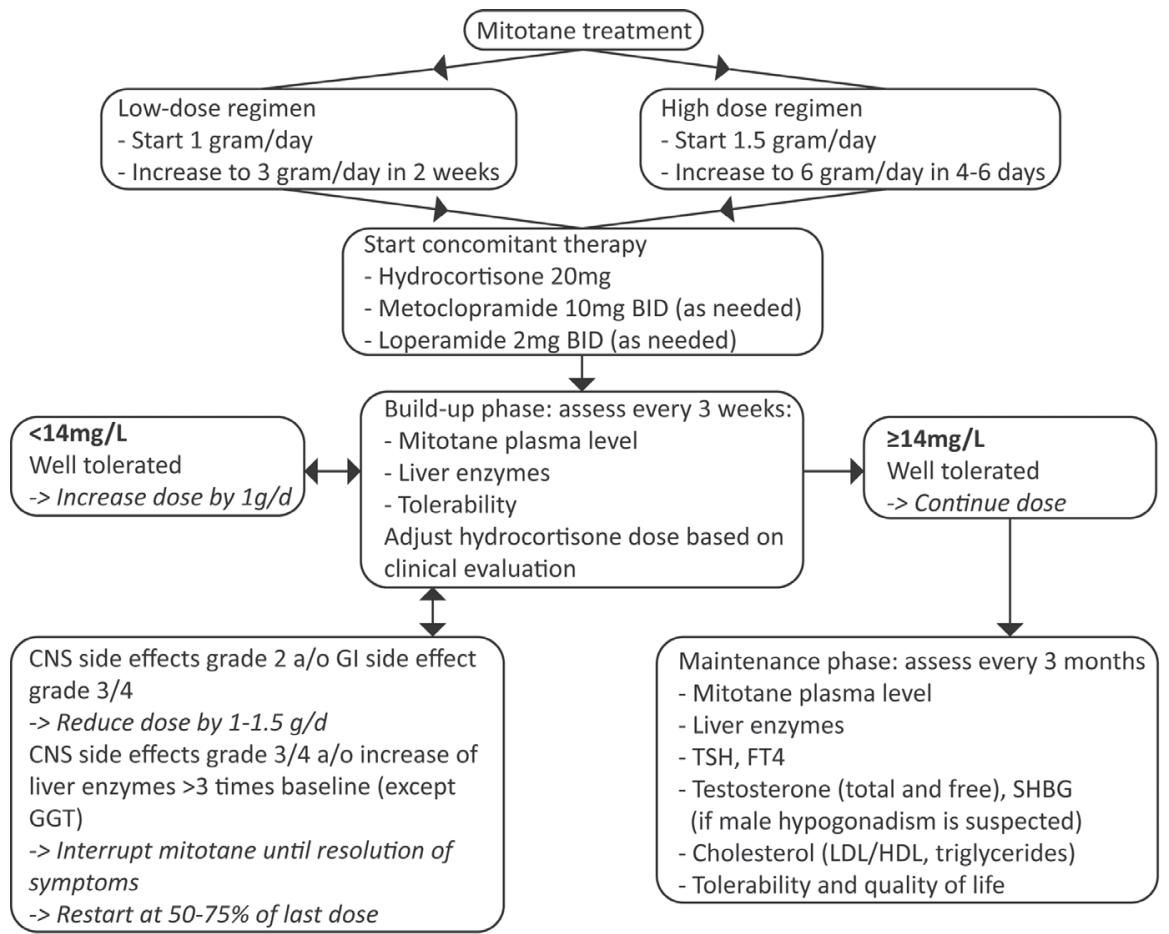




\section{References}

1. Abiven G, Coste J, Groussin L, Anract P, Tissier F, Legmann P, Dousset B, Bertagna X \& Bertherat J 2006 Clinical and biological features in the prognosis of adrenocortical cancer: poor outcome of cortisol-secreting tumors in a series of 202 consecutive patients. The Journal of clinical endocrinology and metabolism 91 2650-2655.

2. Arlt W, Biehl M, Taylor AE, Hahner S, Libe R, Hughes BA, Schneider P, Smith DJ, Stiekema H, Krone $\mathrm{N}$ et al. 2011 Urine steroid metabolomics as a biomarker tool for detecting malignancy in adrenal tumors. The Journal of clinical endocrinology and metabolism 96 3775-3784.

3. Assie G, Antoni G, Tissier F, Caillou B, Abiven G, Gicquel C, Leboulleux S, Travagli JP, Dromain C, Bertagna X et al. 2007 Prognostic parameters of metastatic adrenocortical carcinoma. The Journal of clinical endocrinology and metabolism 92 148-154.

4. Assie G, Letouze E, Fassnacht M, Jouinot A, Luscap W, Barreau O, Omeiri H, Rodriguez S, Perlemoine K, Rene-Corail F et al. 2014 Integrated genomic characterization of adrenocortical carcinoma. Nature genetics 46 607-612.

5. Barreau O, Assie G, Wilmot-Roussel H, Ragazzon B, Baudry C, Perlemoine K, Rene-Corail F, Bertagna X, Dousset B, Hamzaoui N et al. 2013 Identification of a CpG island methylator phenotype in adrenocortical carcinomas. The Journal of clinical endocrinology and metabolism 98 E174-84.

6. Bates SE, Shieh CY, Mickley LA, Dichek HL, Gazdar A, Loriaux DL \& Fojo AT 1991 Mitotane enhances cytotoxicity of chemotherapy in cell lines expressing a multidrug resistance gene ( $\mathrm{mdr}$ $1 /$ P-glycoprotein) which is also expressed by adrenocortical carcinomas. The Journal of clinical endocrinology and metabolism 73 18-29.

7. Baudin E, Docao C, Gicquel C, Vassal G, Bachelot A, Penfornis A \& Schlumberger M 2002 Use of a topoisomerase I inhibitor (irinotecan, CPT-11) in metastatic adrenocortical carcinoma. Annals of Oncology : Official Journal of the European Society for Medical Oncology / ESMO 13 1806-1809.

8. Baudin E, Leboulleux S, Al Ghuzlan A, Chougnet C, Young J, Deandreis D, Dumont F, Dechamps F, Caramella C, Chanson P et al. 2011 Therapeutic management of advanced adrenocortical carcinoma: what do we know in 2011? Hormones \& cancer 2 363-371.

9. Baudin E, Pellegriti G, Bonnay M, Penfornis A, Laplanche A, Vassal G \& Schlumberger M 2001 Impact of monitoring plasma 1,1-dichlorodiphenildichloroethane (o,p'DDD) levels on the treatment of patients with adrenocortical carcinoma. Cancer 92 1385-1392.

10. Bergenstal D, Hertz R, Lipsett M \& Moy R 1960 Chemotherapy of adrenocortical cancer with o,p'DDD. 672.

11. Berruti A, Baudin E, Gelderblom H, Haak HR, Porpiglia F, Fassnacht M, Pentheroudakis G \& on behalf of the ESMO Guidelines Working Group 2012 Adrenal cancer: ESMO Clinical Practice Guidelines for diagnosis, treatment and follow-up. Annals of Oncology 23 vii131-vii138.

12. Berruti A, Fassnacht M, Haak H, Else T, Baudin E, Sperone P, Kroiss M, Kerkhofs T, Williams AR, Ardito A et al. 2014 Prognostic role of overt hypercortisolism in completely operated patients with adrenocortical cancer. European urology 65 832-838.

13. Berruti A, Sperone P, Ferrero A, Germano A, Ardito A, Priola AM, De Francia S, Volante M, Daffara F, Generali D et al. 2011 Phase II study of weekly paclitaxel and sorafenib as second/third line therapy in patients with adrenocortical carcinoma. European journal of endocrinology / European Federation of Endocrine Societies.

14. Berruti A, Terzolo M, Pia A, Angeli A \& Dogliotti L 1998 Mitotane associated with etoposide, doxorubicin, and cisplatin in the treatment of advanced adrenocortical carcinoma. Italian Group for the Study of Adrenal Cancer. Cancer 83 2194-2200. 
15. Berruti A, Terzolo M, Sperone P, Pia A, Casa SD, Gross DJ, Carnaghi C, Casali P, Porpiglia F, Mantero F et al. 2005 Etoposide, doxorubicin and cisplatin plus mitotane in the treatment of advanced adrenocortical carcinoma: a large prospective phase II trial. Endocrine-related cancer 12 657-666.

16. Beuschlein F, Weigel J, Saeger W, Kroiss M, Wild V, Daffara F, Libe R, Ardito A, Al Ghuzlan A, Quinkler $M$ et al. 2015 Major prognostic role of Ki67 in localized adrenocortical carcinoma after complete resection. The Journal of clinical endocrinology and metabolism 100 841-849.

17. Brix D, Allolio B, Fenske W, Agha A, Dralle H, Jurowich C, Langer P, Mussack T, Nies C, Riedmiller H et al. 2010 Laparoscopic versus open adrenalectomy for adrenocortical carcinoma: surgical and oncologic outcome in 152 patients. European urology 58 609-615.

18. Cazejust J, De Baere T, Auperin A, Deschamps F, Hechelhammer L, Abdel-Rehim M, Schlumberger M, Leboulleux S \& Baudin E 2010 Transcatheter arterial chemoembolization for liver metastases in patients with adrenocortical carcinoma. Journal of vascular and interventional radiology : JVIR 21 1527-1532.

19. Chabre O, Libe R, Assie G, Barreau O, Bertherat J, Bertagna X, Feige JJ \& Cherradi N 2013 Serum miR-483-5p and miR-195 are predictive of recurrence risk in adrenocortical cancer patients. Endocrine-related cancer 20 579-594.

20. Chortis V, Taylor AE, Schneider P, Tomlinson JW, Hughes BA, O'Neil DM, Libe R, Allolio B, Bertagna X, Bertherat J et al. 2013 Mitotane therapy in adrenocortical cancer induces CYP3A4 and inhibits 5alpha-reductase, explaining the need for personalized glucocorticoid and androgen replacement. The Journal of clinical endocrinology and metabolism 98 161-171.

21. Cooper AB, Habra MA, Grubbs EG, Bednarski BK, Ying AK, Perrier ND, Lee JE \& Aloia TA 2013 Does laparoscopic adrenalectomy jeopardize oncologic outcomes for patients with adrenocortical carcinoma? Surgical endoscopy 27 4026-4032.

22. Daffara F, De Francia S, Reimondo G, Zaggia B, Aroasio E, Porpiglia F, Volante M, Termine A, Di Carlo F, Dogliotti L et al. 2008 Prospective evaluation of mitotane toxicity in adrenocortical cancer patients treated adjuvantly. Endocrine-related cancer 15 1043-1053.

23. D'Avolio A, De Francia S, Basile V, Cusato J, De Martino F, Pirro E, Piccione F, Ardito A, Zaggia B, Volante M et al. 2013 Influence of the CYP2B6 polymorphism on the pharmacokinetics of mitotane. Pharmacogenetics and genomics.

24. De Reynies A, Assie G, Rickman DS, Tissier F, Groussin L, Rene-Corail F, Dousset B, Bertagna X, Clauser E \& Bertherat J 2009 Gene expression profiling reveals a new classification of adrenocortical tumors and identifies molecular predictors of malignancy and survival. Journal of clinical oncology : official journal of the American Society of Clinical Oncology 27 1108-1115.

25. Donatini G, Caiazzo R, Do Cao C, Aubert S, Zerrweck C, El-Kathib Z, Gauthier T, Leteurtre E, Wemeau JL, Vantyghem MC et al. 2014 Long-term survival after adrenalectomy for stage I/II adrenocortical carcinoma (ACC): a retrospective comparative cohort study of laparoscopic versus open approach. Annals of surgical oncology 21 284-291.

26. Dy BM, Strajina V, Cayo AK, Richards ML, Farley DR, Grant CS, Harmsen WS, Evans DB, Grubbs EG, Bible KC et al. 2015 Surgical resection of synchronously metastatic adrenocortical cancer. Annals of surgical oncology 22 146-151.

27. Erdogan I, Deutschbein T, Jurowich C, Kroiss M, Ronchi C, Quinkler M, Waldmann J, Willenberg HS, Beuschlein F, Fottner C et al. 2013 The role of surgery in the management of recurrent adrenocortical carcinoma. The Journal of clinical endocrinology and metabolism 98 181-191.

28. Faggiano A, Leboulleux S, Young J, Schlumberger M \& Baudin E 2006 Rapidly progressing high o,p'DDD doses shorten the time required to reach the therapeutic threshold with an acceptable tolerance: preliminary results. Clinical endocrinology 64 110-113. 
29. Fassnacht M \& Allolio B 2009 Clinical management of adrenocortical carcinoma. Best.Pract.Res. Clin Endocrinol.Metab 23 273-289.

30. Fassnacht M, Berruti A, Baudin E, Demeure MJ, Gilbert J, Haak H, Kroiss M, Quinn DI, Hesseltine E, Ronchi CL et al. 2015 Linsitinib (OSI-906) versus placebo for patients with locally advanced or metastatic adrenocortical carcinoma: a double-blind, randomised, phase 3 study. The Lancet. Oncology 16 426-435.

31. Fassnacht M, Hahner S, Polat B, Koschker AC, Kenn W, Flentje M \& Allolio B 2006 Efficacy of adjuvant radiotherapy of the tumor bed on local recurrence of adrenocortical carcinoma. The Journal of clinical endocrinology and metabolism 91 4501-4504.

32. Fassnacht M, Johanssen S, Fenske W, Weismann D, Agha A, Beuschlein F, Fuhrer D, Jurowich C, Quinkler M, Petersenn S et al. 2010 Improved survival in patients with stage II adrenocortical carcinoma followed up prospectively by specialized centers. The Journal of clinical endocrinology and metabolism 95 4925-4932.

33. Fassnacht M, Johanssen S, Quinkler M, Bucsky P, Willenberg HS, Beuschlein F, Terzolo M, Mueller $\mathrm{HH}$, Hahner S, Allolio B et al. 2009 Limited prognostic value of the 2004 International Union Against Cancer staging classification for adrenocortical carcinoma: proposal for a Revised TNM Classification. Cancer 115 243-250.

34. Fassnacht M, Libe R, Kroiss M \& Allolio B 2011 Adrenocortical carcinoma: a clinician's update. Nature reviews. Endocrinology 7 323-335.

35. Fassnacht M, Terzolo M, Allolio B, Baudin E, Haak H, Berruti A, Welin S, Schade-Brittinger C, Lacroix A, Jarzab B et al. 2012 Combination Chemotherapy in Advanced Adrenocortical Carcinoma. N Engl J Med.

36. Fay AP, Signoretti S, Callea M, Telomicron GH, McKay RR, Song J, Carvo I, Lampron ME, Kaymakcalan MD, Poli-de-Figueiredo CE et al. 2015 Programmed death ligand-1 expression in adrenocortical carcinoma: an exploratory biomarker study. Journal for immunotherapy of cancer 3 3-015-0047-3. eCollection 2015.

37. Finks JF, Osborne NH \& Birkmeyer JD 2011 Trends in hospital volume and operative mortality for high-risk surgery. The New England journal of medicine 364 2128-2137.

38. Fraenkel M, Gueorguiev M, Barak D, Salmon A, Grossman AB \& Gross DJ 2013 Everolimus therapy for progressive adrenocortical cancer. Endocrine 44 187-192.

39. Fukushima DK, Bradlow HL \& Hellman L 1971 Effects of o,p'-DDD on cortisol and 6-betahydroxycortisol secretion and metabolism in man. The Journal of clinical endocrinology and metabolism 32 192-200.

40. Ghataore L, Chakraborti I, Aylwin SJ, Schulte KM, Dworakowska D, Coskeran P \& Taylor NF 2012 Effects of mitotane treatment on human steroid metabolism: implications for patient management. Endocrine connections $137-47$.

41. Giordano TJ, Kuick R, Else T, Gauger PG, Vinco M, Bauersfeld J, Sanders D, Thomas DG, Doherty G \& Hammer G 2009 Molecular classification and prognostication of adrenocortical tumors by transcriptome profiling. Clinical cancer research : an official journal of the American Association for Cancer Research 15 668-676.

42. Gross DJ, Munter G, Bitan M, Siegal T, Gabizon A, Weitzen R, Merimsky O, Ackerstein A, Salmon A, Sella A et al. 2006 The role of imatinib mesylate (Glivec) for treatment of patients with malignant endocrine tumors positive for c-kit or PDGF-R. Endocrine-related cancer 13 535-540.

43. Grubbs EG, Callender GG, Xing Y, Perrier ND, Evans DB, Phan AT \& Lee JE 2010 Recurrence of adrenal cortical carcinoma following resection: surgery alone can achieve results equal to surgery plus mitotane. Annals of Surgical Oncology: The Official Journal of the Society of Surgical Oncology 17 263-270. 
44. Haak HR, Hermans J, van de Velde CJ, Lentjes EG, Goslings BM, Fleuren GJ \& Krans HM 1994 Optimal treatment of adrenocortical carcinoma with mitotane: results in a consecutive series of 96 patients. British journal of cancer 69 947-951.

45. Habra MA, Ejaz S, Feng L, Das P, Deniz F, Grubbs EG, Phan A, Waguespack SG, Ayala-Ramirez M, Jimenez C et al. 2013 A retrospective cohort analysis of the efficacy of adjuvant radiotherapy after primary surgical resection in patients with adrenocortical carcinoma. The Journal of clinical endocrinology and metabolism 98 192-197.

46. Hahner S, KreissI MC, Fassnacht M, Haenscheid H, Knoedler P, Lang K, Buck AK, Reiners C, Allolio B \& Schirbel A 2011 [1311]lodometomidate for Targeted Radionuclide Therapy of Advanced Adrenocortical Carcinoma. Journal of Clinical Endocrinology \& Metabolism.

47. Hermsen IG, Gelderblom H, Kievit J, Romijn JA \& Haak HR 2008 Extremely long survival in six patients despite recurrent and metastatic adrenal carcinoma. European journal of endocrinology / European Federation of Endocrine Societies 158 911-919.

48. Hermsen IG, Groenen YE, Dercksen MW, Theuws J \& Haak HR 2010 Response to Radiation Therapy in Adrenocortical Carcinoma. Journal of endocrinological investigation.

49. Hermsen IG, Kerkhofs TM, Butter G, Kievit J, van Eijck CH, Nieveen van Dijkum EJ, Haak HR \& Dutch Adrenal Network 2012 Surgery in adrenocortical carcinoma: Importance of national cooperation and centralized surgery. Surgery 152 50-56.

50. Ho V, de Raaf A, van der Hoeven K \& Jansen-Landheer M 2012 IKNL berekent effect volumenormen op ziekenhuizen en patiënten. Medisch Contact $\mathbf{5 0}$

51. Ho J, Turkbey B, Edgerly M, Alimchandani M, Quezado M, Camphausen K, Fojo T \& Kaushal A 2013 Role of radiotherapy in adrenocortical carcinoma. Cancer journal (Sudbury, Mass.) 19 288-294.

52. Jurowich C, Fassnacht M, Kroiss M, Deutschbein T, Germer CT \& Reibetanz J 2013 Is there a role for laparoscopic adrenalectomy in patients with suspected adrenocortical carcinoma? A critical appraisal of the literature. Hormone and metabolic research = Hormon- und Stoffwechselforschung = Hormones et metabolisme 45 130-136.

53. Kebebew E, Reiff E, Duh QY, Clark OH \& McMillan A 2006 Extent of disease at presentation and outcome for adrenocortical carcinoma: have we made progress? World journal of surgery 30872 878.

54. Kerkhofs TM, Baudin E, Terzolo M, Allolio B, Chadarevian R, Mueller HH, Skogseid B, Leboulleux S, Mantero F, Haak HR et al. 2013a Comparison of two mitotane starting dose regimens in patients with advanced adrenocortical carcinoma. The Journal of clinical endocrinology and metabolism 98 4759-4767.

55. Kerkhofs TM, Derijks LJ, Ettaieb H, den Hartigh J, Neef K, Gelderblom H, Guchelaar HJ \& Haak HR 2015a Development of a pharmacokinetic model of mitotane: toward personalized dosing in adrenocortical carcinoma. Therapeutic drug monitoring 37 58-65.

56. Kerkhofs TM, Kerstens MN, Kema IP, Willems TP \& Haak HR 2015b Diagnostic Value of Urinary Steroid Profiling in the Evaluation of Adrenal Tumors. Hormones \& cancer.

57. Kerkhofs TM, Verhoeven RH, Bonjer HJ, van Dijkum EJ, Vriens MR, De Vries J, Van Eijck CH, Bonsing BA, Van de Poll-Franse LV, Haak HR et al. 2013b Surgery for adrenocortical carcinoma in The Netherlands: analysis of the national cancer registry data. European journal of endocrinology / European Federation of Endocrine Societies 169 83-89.

58. Kerkhofs TM, Verhoeven RH, Van der Zwan JM, Dieleman J, Kerstens MN, Links TP, Van de PollFranse LV \& Haak HR 2013c Adrenocortical carcinoma: a population-based study on incidence and survival in the Netherlands since 1993. European journal of cancer (Oxford, Eng/and: 1990) 49 2579-2586. 
59. Kroiss M, Quinkler M, Johanssen S, van Erp NP, Lankheet N, Pollinger A, Laubner K, Strasburger C), Hahner S, Muller HH et al. 2012 Sunitinib in refractory adrenocortical carcinoma: a phase II, single-arm, open-label trial. The Journal of clinical endocrinology and metabolism 97 3495-3503.

60. Kroiss M, Quinkler M, Lutz WK, Allolio B \& Fassnacht M 2011 Drug interactions with mitotane by induction of CYP3A4 metabolism in the clinical management of adrenocortical carcinoma. Clinical endocrinology 75 585-591.

61. Libe R, Borget I, Ronchi CL, Ardito A, Kroiss M, Bertherat J, Quinkler M, Bala M, Beuschlein F, Vezzosi D et al. 2015 Prognostic factors of overall survival of stage III or IV adrenocortical carcinomas (ACC): A multicenter ENS@T study. Annals of Oncology (in press).

62. Lombardi CP, Raffaelli M, De Crea C, Boniardi M, De Toma G, Marzano LA, Miccoli P, Minni F, Morino M, Pelizzo MR et al. 2012 Open versus endoscopic adrenalectomy in the treatment of localized (stage I/II) adrenocortical carcinoma: results of a multiinstitutional Italian survey. Surgery $1521158-1164$.

63. Lubitz JA, Freeman L \& Okun R 1973 Mitotane use in inoperable adrenal cortical carcinoma. JAMA : the journal of the American Medical Association 223 1109-1112.

64. Lughezzani G, Sun M, Perrotte P, Jeldres C, Alasker A, Isbarn H, Budaus L, Shariat SF, Guazzoni G, Montorsi F et al. 2010 The European Network for the Study of Adrenal Tumors staging system is prognostically superior to the international union against cancer-staging system: a North American validation. European journal of cancer (Oxford, England : 1990) 46 713-719.

65. McNicol AM, Struthers AL, Nolan CE, Hermans J \& Haak HR 1997 Proliferation in Adrenocortical Tumors: Correlation with Clinical Outcome and p53 Status. Endocrine pathology 8 29-36.

66. Miller BS, Ammori JB, Gauger PG, Broome JT, Hammer GD \& Doherty GM 2010 Laparoscopic Resection is Inappropriate in Patients with Known or Suspected Adrenocortical Carcinoma. World journal of surgery.

67. Miller BS, Gauger PG, Hammer GD \& Doherty GM 2012 Resection of adrenocortical carcinoma is less complete and local recurrence occurs sooner and more often after laparoscopic adrenalectomy than after open adrenalectomy. Surgery 152 1150-1157.

68. Mir MC, KlinkJC, Guillotreau J, Long JA, Miocinovic R, KaoukJH, Simmons MN, Klein E, Krishnamurthi V, Campbell SC et al. 2013 Comparative outcomes of laparoscopic and open adrenalectomy for adrenocortical carcinoma: single, high-volume center experience. Annals of surgical oncology 20 1456-1461.

69. Moolenaar AJ, van SH, van Seters AP \& Smeenk D 1981 Blood levels of o,p'-DDD following administration in various vehicles after a single dose and during long-term treatment. Cancer chemotherapy and pharmacology 7 51-54.

70. Morimoto R, Satoh F, Murakami O, Suzuki T, Abe T, Tanemoto M, Abe M, Uruno A, Ishidoya S, Arai $Y$ et al. 2008 Immunohistochemistry of a proliferation marker Ki67/MIB1 in adrenocortical carcinomas: Ki67/MIB1 labeling index is a predictor for recurrence of adrenocortical carcinomas. Endocrine journal 55 49-55.

71. Perna V, Taylor NF, Dworakowska D, Schulte KM, Aylwin S, Al-Hashimi F \& Diaz-Cano SJ 2014 Adrenocortical adenomas with regression and myelolipomatous changes: urinary steroid profiling supports a distinctive benign neoplasm. Clinical endocrinology 81 343-349.

72. Pinzani P, Scatena C, Salvianti F, Corsini E, Canu L, Poli G, Paglierani M, Piccini V, Pazzagli M, Nesi $\mathrm{G}$ et al. 2013 Detection of circulating tumor cells in patients with adrenocortical carcinoma: a monocentric preliminary study. The Journal of clinical endocrinology and metabolism 98 3731-3738.

73. Polat B, Fassnacht M, Pfreundner L, Guckenberger M, Bratengeier K, Johanssen S, Kenn W, Hahner S, Allolio B \& Flentje M 2009 Radiotherapy in adrenocortical carcinoma. Cancer 115 2816-2823. 
74. Porpiglia F, Fiori C, Daffara F, Zaggia B, Bollito E, Volante M, Berruti A \& Terzolo M 2010 Retrospective evaluation of the outcome of open versus laparoscopic adrenalectomy for stage I and II adrenocortical cancer. European urology 57 873-878.

75. Pucci E, Papathomas TG, Giordano TJ, Lu H, Duregon E, Volante M, Papotti M, Lloyd R, Tischler A \& Nose V 2014 An international Ki-67 reproducibility study in adrenocortical cancer: a multicenter interobserver variation analysis using virtual microscopy. Abstract on 13th scientific meeting of ENS@T, Nice, France.

76. Quinkler M, Hahner S, Wortmann S, Johanssen S, Adam P, Ritter C, Strasburger C, Allolio B \& Fassnacht M 2008 Treatment of advanced adrenocortical carcinoma with erlotinib plus gemcitabine. The Journal of clinical endocrinology and metabolism 93 2057-2062.

77. Reibetanz J, Jurowich C, Erdogan I, Nies C, Rayes N, Dralle H, Behrend M, Allolio B, Fassnacht M \& German ACC study group 2012 Impact of lymphadenectomy on the oncologic outcome of patients with adrenocortical carcinoma. Annals of Surgery 255 363-369.

78. Saade N, Sadler C \& Goldfarb M 2015 Impact of Regional Lymph Node Dissection on Disease Specific Survival in Adrenal Cortical Carcinoma. Hormone and metabolic research $=$ Hormon- und Stoffwechselforschung = Hormones et metabolisme.

79. Sabolch A, Feng M, Griffith K, Hammer G, Doherty G \& Ben-Josef E 2011 Adjuvant and definitive radiotherapy for adrenocortical carcinoma. International journal of radiation oncology, biology, physics 80 1477-1484.

80. Salama JK \& Milano MT 2014 Radical irradiation of extracranial oligometastases. Journal of clinical oncology : official journal of the American Society of Clinical Oncology 32 2902-2912.

81. Sasano H, Suzuki \& \& Moriya T 2006 Recent advances in histopathology and immunohistochemistry of adrenocortical carcinoma. Endocrine pathology 17 345-354.

82. Sbiera S, Kroiss M, Thamm T, Beyer M, Majidi F, Kuehner D, Wobser M, Becker JC, Adam P, Ronchi C et al. 2013 Survivin in adrenocortical tumors - pathophysiological implications and therapeutic potential. Hormone and metabolic research $=$ Hormon- und Stoffwechselforschung $=$ Hormones et metabolisme 45 137-146.

83. Schulick RD \& Brennan MF 1999 Long-term survival after complete resection and repeat resection in patients with adrenocortical carcinoma. Annals of Surgical Oncology: The Official Journal of the Society of Surgical Oncology 6 719-726.

84. Southren AL, Tochimoto S, Isurugi K, Gordon GG, Krikun E \& Stypulkowski W 1966 The effect of 2,2-bis (2-chlorophenyl-4-chlorophenyl)-1,1-dichloroethane (o, $p^{\prime}$-DDD) on the metabolism of infused cortisol-7-3H. Steroids 7 11-29.

85. Sperone P, Ferrero A, Daffara F, Priola A, Zaggia B, Volante M, Santini D, Vincenzi B, Badalamenti G, Intrivici C et al. 2010 Gemcitabine plus metronomic 5-fluorouracil or capecitabine as a second-/ third-line chemotherapy in advanced adrenocortical carcinoma: a multicenter phase II study. Endocrine-related cancer 17 445-453.

86. Sun YF, Yang XR, Zhou J, Qiu SJ, Fan J \& Xu Y 2011 Circulating tumor cells: advances in detection methods, biological issues, and clinical relevance. Journal of cancer research and clinical oncology $1371151-1173$.

87. Terzolo M, Angeli A, Fassnacht M, Daffara F, Tauchmanova L, Conton PA, Rossetto R, Buci L, Sperone P, Grossrubatscher E et al. 2007 Adjuvant mitotane treatment for adrenocortical carcinoma. The New England journal of medicine 356 2372-2380.

88. Terzolo M, Boccuzzi A, Bovio S, Cappia S, De Giuli P, Ali A, Paccotti P, Porpiglia F, Fontana D \& Angeli A 2001 Immunohistochemical assessment of Ki-67 in the differential diagnosis of adrenocortical tumors. Urology 57 176-182. 
89. Terzolo M, Pia A, Berruti A, Osella G, Ali A, Carbone V, Testa E, Dogliotti L \& Angeli A 2000 Low-dose monitored mitotane treatment achieves the therapeutic range with manageable side effects in patients with adrenocortical cancer. The Journal of clinical endocrinology and metabolism 852234 2238.

90. Toniato A 2013 Minimally invasive surgery for malignant adrenal tumors. The surgeon : journal of the Royal Colleges of Surgeons of Edinburgh and Ireland 11 253-257.

91. Topalian SL, Hodi FS, Brahmer JR, Gettinger SN, Smith DC, McDermott DF, Powderly JD, Carvajal RD, Sosman JA, Atkins MB et al. 2012 Safety, activity, and immune correlates of anti-PD-1 antibody in cancer. The New England journal of medicine 366 2443-2454.

92. Touitou Y, Bogdan A \& Luton JP 1978 Changes in corticosteroid synthesis of the human adrenal cortex in vitro, induced by treatment with o,p'-DDD for Cushing's syndrome: evidence for the sites of action of the drug. Journal of steroid biochemistry 9 1217-1224.

93. Tran TB, Liou D, Menon VG \& Nissen NN 2013 Surgical management of advanced adrenocortical carcinoma: a 21-year population-based analysis. The American Surgeon 79 1115-1118.

94. Urup T, Pawlak WZ, Petersen PM, Pappot H, Rorth M \& Daugaard G 2013 Treatment with docetaxel and cisplatin in advanced adrenocortical carcinoma, a phase II study. British journal of cancer 108 1994-1997.

95. van Erp NP, Guchelaar HJ, Ploeger BA, Romijn JA, Hartigh J \& Gelderblom H 2011 Mitotane has a strong and a durable inducing effect on CYP3A4 activity. European journal of endocrinology / European Federation of Endocrine Societies 164 621-626.

96. von Slooten H, van Seters AP, Smeenk D \& Moolenaar AJ 1982 O,p'-DDD (mitotane) levels in plasma and tissues during chemotherapy and at autopsy. Cancer chemotherapy and pharmacology 9 85-88.

97. Wood BJ, Abraham J, Hvizda JL, Alexander HR \& Fojo T 2003 Radiofrequency ablation of adrenal tumors and adrenocortical carcinoma metastases. Cancer 97 554-560.

98. Wortmann S, Quinkler M, Ritter C, Kroiss M, Johanssen S, Hahner S, Allolio B \& Fassnacht M 2010 Bevacizumab plus capecitabine as a salvage therapy in advanced adrenocortical carcinoma. European journal of endocrinology / European Federation of Endocrine Societies 162 349-356.

99. Zatelli MC, Gentilin E, Daffara F, Tagliati F, Reimondo G, Carandina G, Ambrosio MR, Terzolo M \& Degli Uberti EC 2010 Therapeutic concentrations of mitotane (o,p'-DDD) inhibit thyrotroph cell viability and TSH expression and secretion in a mouse cell line model. Endocrinology $1512453-$ 2461. 
5 


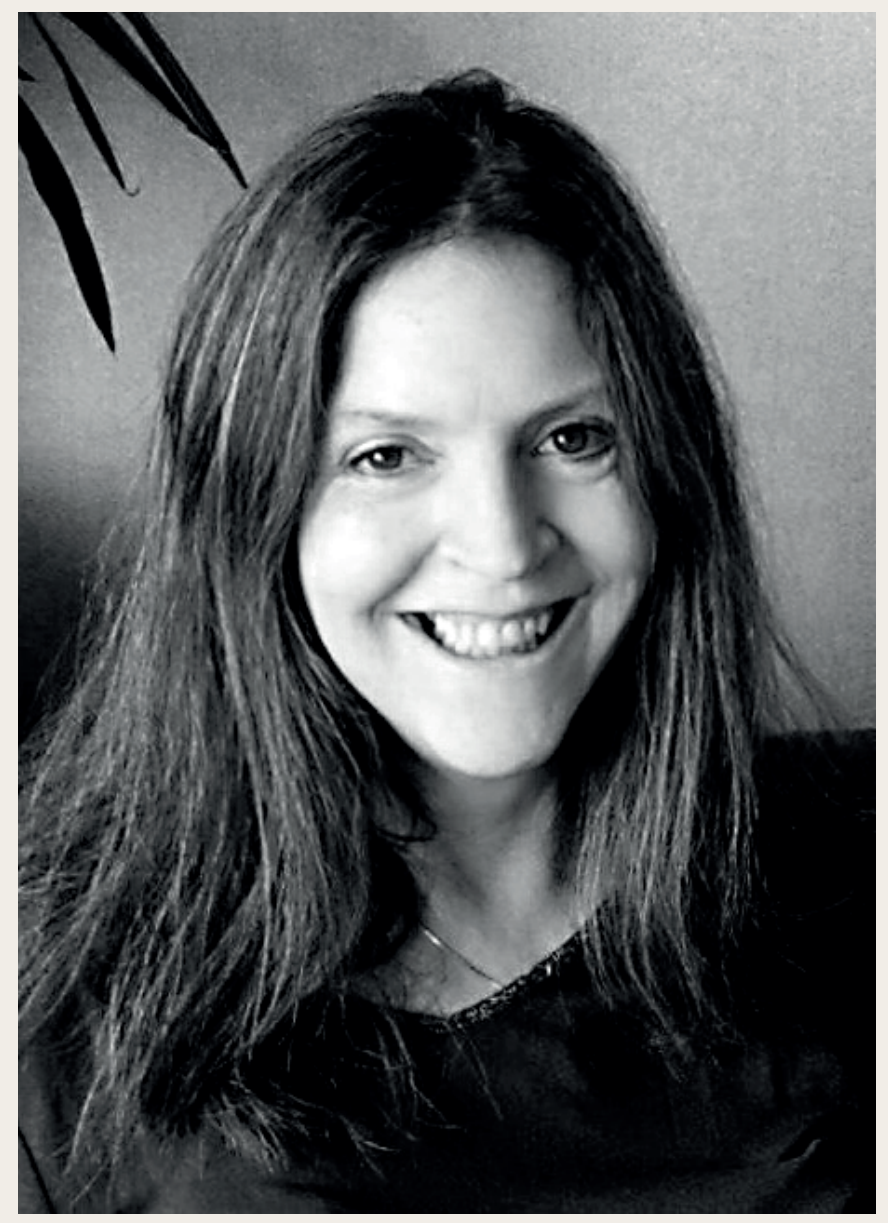




\section{Chapter 06}

\section{Past, Present and Future of Epigenetics in Adrenocortical Carcinoma}

Madeleine Ettaieb, Thomas Kerkhofs, Manon van Engeland and Harm Haak Cancers. 2020 May 13;12(5):E1218 


\section{Abstract}

DNA methylation profiling has been suggested a reliable technique to distinguish between benign and malignant adrenocortical tumors, a process which with current diagnostic methods remains challenging and lacks diagnostic accuracy of borderline tumors. Accurate distinction between benign and malignant adrenal tumors is of the essence, since ACC is a rare but aggressive endocrine disease with an annual incidence of about 2.0 cases per million people per year. The estimated five-year overall survival rate for ACC patients is $<50 \%$. However, available treatment regimens are limited, in which a radical surgical resection is the only curable option. Nevertheless, up to $85 \%$ of patients with radical resection show recurrence of the local disease often with concurrent metastases. Adrenolytic therapy with mitotane, administered alone or in combination with cytotoxic agents, is currently the primary (palliative) treatment for patients with advanced ACC and is increasingly used in adjuvant setting to prevent recurrence. Prognostic stratification is important in order to individualize adjuvant therapies. On April 1, 2020, there were 7404 publications on adrenocortical carcinoma ((c(((adrenocortical carcinoma) OR adrenocortical carcinoma [MeSH Terms]) OR adrenal cortex cancer[MeSH Terms]) OR adrenal cortical carcinoma [MeSH Terms]) OR adrenal cortex neoplasm [MeSH Terms]) OR adrenocortical cancer [MeSH Terms]), yet the underlying pathophysiology and characteristics of ACC is not fully understood. Knowledge on epigenetic alterations in the process of adrenal tumorigenesis is rapidly increasing and will add to a better understanding of the pathogenesis of ACC. DNA methylation profiling has been heralded as a promising method in the prognostication of ACC. This review summarizes recent findings on epigenetics of ACC and its role in diagnosis, prognosis and therapeutic strategies.

Keywords: adrenocortical carcinoma; epigenetics; DNA methylation 


\section{Introduction}

Adrenocortical tumors (ACTs) are frequently discovered as incidentaloma due to increased use of imaging in a variety of medical settings. The first computed tomography (CT) series published in the early 1980s showed a prevalence of adrenal incidentaloma between 0.7-1.3\% [1,2]. In a series published between 1982 to 1994 the mean prevalence of adrenal incidentaloma was $0.64 \%$ (ranging from $0.35-1.9 \%$ ) [3], whereas, in 1991 Herrera et al. showed that only $0.4 \%$ of all the CT scans showed serendipitously discovered adrenal masses [4]). Bovio et al. showed a prevalence of 4.4\% in their series of high resolution CT scans [5].

Most of these incidentalomas are benign adrenocortical adenomas (ACA), nonfunctional and clinically irrelevant. Their malignant counterpart, adrenocortical carcinoma (ACC), is a rare and aggressive type of cancer. Although estimates varied widely, the frequency of primary adrenal carcinoma in patients with adrenal incidentaloma ranges from 1.2-11\% [6]. It should be kept in mind that due to the nature of these studies, selection bias is very probable (the populations studied not reflecting a random sample of all patients with an adrenal incidentaloma) and most likely leads to an overestimation of the frequency of ACC. Differentiating between these two types of tumors can be challenging, considering that clinical, laboratory, radiological and even histological features may overlap. Although ACC occurs in children (only $0.2 \%$ of pediatric cancers [7], annual incidence of 0.2 to 0.3 cases per 1 million individuals [8]), most cases appear between ages 30 and 50 (0.02\% to $0.2 \%$ of adult cancers [9], annual incidence of 0.5-2.0 patient per million people per year [10]. An exception to these epidemiologic data is described in southern Brazil, where the annual incidence of adrenal cancer in children is unusually high, ranging from 3.4-4.2 per million children [11]. The distribution of tumors seems to follow a regional rather than familial pattern, therefore environmental factors have been considered, but so far none have been identified. 


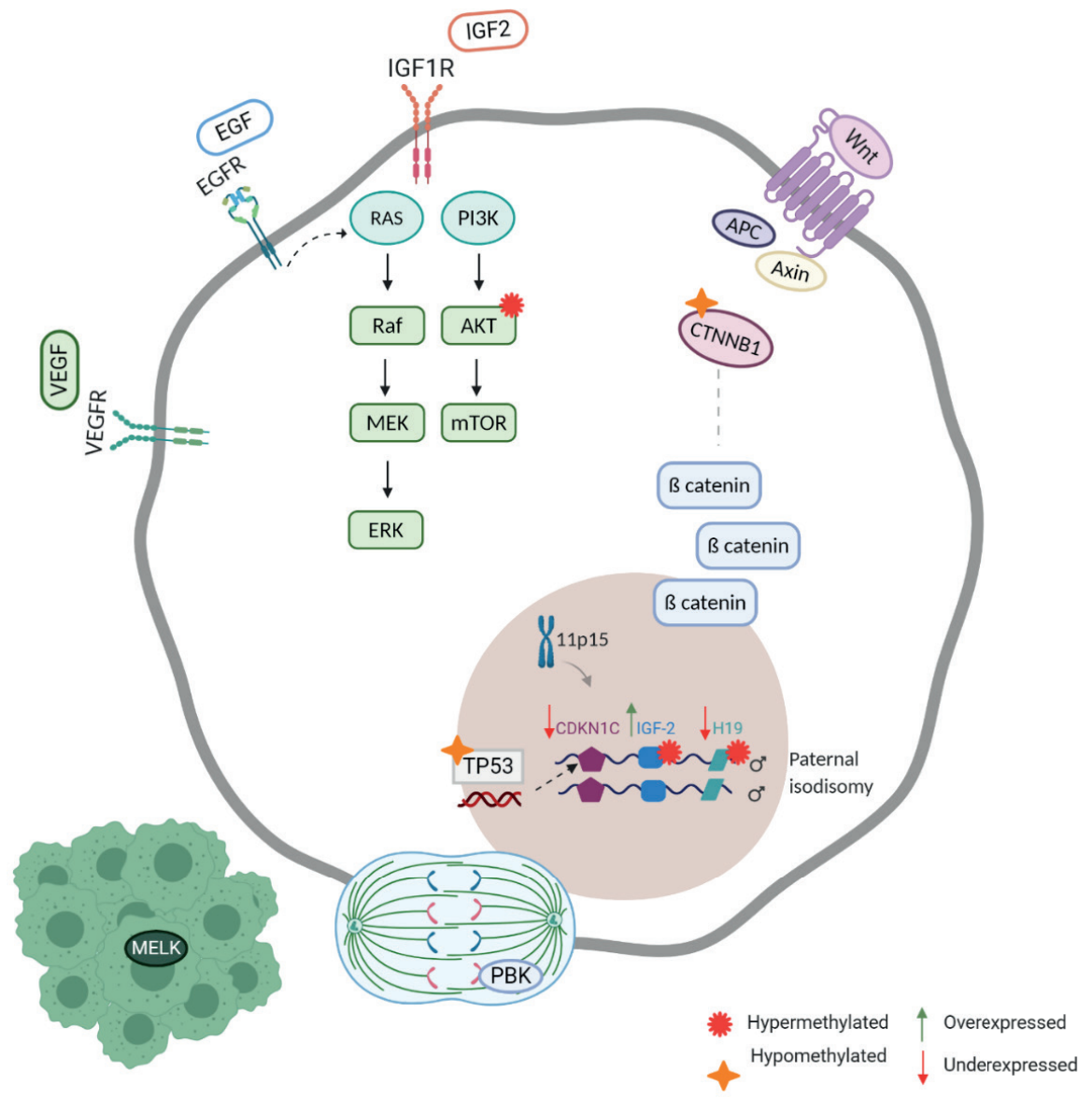

Figure 1. Major dysfunctional molecular pathways in adrenocortical carcinoma, resulting in abnormal survival, proliferation, apoptosis resistance, metastasis and angiogenesis [Created with BioRender.com].

\subsection{Molecular Alterations}

The understanding of adrenocortical tumorigenesis has been challenging since molecular studies on adrenocortical carcinoma have been based on a small number of samples. Until recently they have been directed mainly to candidate genes. It was with this approach that the first genetic studies on ACC started with the elucidation of rare genetic syndromes (e.g. Li Fraumeni syndrome, Beckwith Wiedemann syndrome) in which ACTs are a manifestation [12]. They have led to the discovery of major dysfunctional molecular pathways in adrenocortical tumors, such as the IGF pathway, the Wht pathway and TP53 (Figure 1) [12]. TP53 germline mutations have been described with a mutation prevalence of 3.9\% [13]. 
Gene expression analysis showed that IGF2, a fetal growth factor imprinted at chromosome 11p15 locus, is upregulated and overexpressed in ACC. Another gene at the 11p15 locus, H19 (a non-protein coding RNA) associated with the inhibition of IGF2 expression, is under expressed in ACC [14].

In ACC, the Wnt/B-catenin (CTNNB1) is frequently activated trough CTNNB1 mutations and even associated with a poor outcome [15]. In approximately 25\% of both benign and malignant sporadic adrenocortical neoplasms $\beta$-catenin gain-of-function mutations are evident [16]. Zheng et al (2016) found 41\% of ACC cases to have alterations of ZNRF3, CTNNB1, APC and MEN1 resulting in modification of the Wnt/ $\beta$ catenin pathway [17].

Tyrosine-kinase coupled receptors have been confirmed to be abnormally active in the IGF pathway, the epidermal growth factor (EGF), fibroblast growth factor (FGF) and vascular endothelial growth factor (VEGF) pathway. These pathways are associated with cell survival, proliferation, angiogenesis, apoptosis resistance and metastasis [12].

Recently, efforts have been made to overcome the problem of small number of ACC samples by developing new preclinical models (CU-ACC1 and CU-ACC2) to advance ACC research [18]. For a long time H295R, initially established in 1980 from a 48-yearold female patient diagnosed with ACC, was the only cell line available for research [19]. With these new preclinical models Kiseljak-Vassiliades et al. have attempted to find new therapies by focusing on identifying the cell cycle kinases in ACC and pinpointing defects in the DNA damage response pathway. They analyzed publicly available expression data sets and observed that maternal embryonic leucine zipper kinase (MELK) was one of the most upregulated kinases in adrenal cancer compared to normal tissue [20].

This research group also observed the mitotic PDZ-binding kinase (PBK; also known as T-lymphokine-activated killer cell-originated protein kinase (TOPK)), a master mitotic kinase known for its role in mitotic division and regulation, to be highly overexpressed in ACC tissues compared to normal adrenal samples [21].

\subsection{Prognostication}

Clinical behavior among ACCs is heterogeneous and stage dependent. The extent of the disease at the time of diagnosis is best assessed by the European Network for the Study of Adrenal Tumor (ENSAT) staging score (Table 1) [22]. 
Table 1. ENSAT score.

\begin{tabular}{ll}
\hline Stage & TNM \\
\hline I & T1,N0,M0 Tumor $\leq 5 \mathrm{~cm}$ \\
II & T2,NO,MO $>5 \mathrm{~cm}$ \\
IV & T1-2,N1,M0 or T3-4,NO-1,M0 \\
\hline
\end{tabular}

T, tumor. $\mathrm{N}$, lymph node. $\mathrm{M}$, metastasis. $\mathrm{T} 1$, tumor size $\leq 5$ centimeter $(\mathrm{cm}) ; \mathrm{T} 2$, tumor size $>5 \mathrm{~cm}$, T3, tumor infiltration in surrounding tissue; T4, tumor invasion in adjacent organs or venous tumor thrombus in vena cava or renal vein. N0, no positive lymph nodes; N1, positive lymph node(s). M0, no distant metastases; M1, presence of distant metastasis.

Prognosis in stage IV disease can vary from a few months to many years [23]. The immunohistochemical Ki67 index $<10 \%$ has been correlated with a relatively good prognosis where an index over 20\% correlates with a more grim prognosis [24]. Despite the high inter- and intra-observer variability [25], Ki67 is currently the most accepted and used prognostic parameter [26] as confirmed by five studies [2731]. It is recommended in the guidelines to use the Ki67 immunohistochemistry for every resection specimen of an adrenocortical tumor, and therapeutic strategies are suggested based on low-risk (Ki67 $\leq 10 \%$ ) or high-risk (Ki67 >10\%) stratification [32]. However, the ACC guideline does not provide directions for a standardized pre-analytical process or scoring approach for Ki67. There is a need for new reliable and reproductive diagnostic tests that can add to current classification scores or even outperform them and correlate better with biological behavior. It is currently not possible to predict whether a patient is cured after complete surgery commonly resulting in the prescription of adjuvant mitotane although some patients may not benefit from it. Over 20\% of patients with tumor stages I to III die within the first 3 years, opting for a more aggressive post-operative systemic treatment. Furthermore, long-term survival of patients with metastatic disease at diagnosis has been described [23]. It is relevant to understand which patient with stage IV disease at primary diagnosis should be offered a treatment strategy facilitating such a long-term survival, or for which patients therapy should be aimed at quality of life and comfort [22].

Genomic profiling approaches have proven to be able to differentiate between adenomas and carcinomas and also to differentiate between carcinomas with different clinical outcome $[33,34]$. De Reynies et al. specified a malignant molecular cluster C1A and C1B versus a benign molecular cluster C2. Tumors of the C1A group had a very poor outcome and were enriched in transcription and mitotic cell cycle genes, whereas the good prognosis malignant C1B group was enriched in cell metabolism, intracellular transport, apoptosis and cell differentiation genes [34]. 
Giordano et al. showed a cluster analysis of the ACCs which revealed two subtypes that reflected tumor proliferation, as measured by mitotic counts and cell cycle genes [33]. Such accurate distinction is essential since treatment is radically different between adenoma and carcinomas. We were unable to find a study that compared a genomic profile versus regular immunohistochemical classification to differentiate between adrenal adenoma and carcinoma, therefore it is not clear whether genomics is superior to pathology.

Giordano showed that pathologists accurately classify the large majority of ACTs using traditional clinicopathologic techniques, yet concluded that occasionally ACTS pose diagnostic challenges and would benefit from additional approaches and tools [35]. Lippert et al. found an improved prognostic stratification when implementing a modified Grading (G; ENSAT and Ki67), resection status (R), Age (A) and as tumor- or hormone-related symptoms (S) (mGRAS) system, by recognizing four ACC subgroups with a different clinical outcome by merging mGRAS and a molecular score (number of somatic mutations, alterations in the Wnt/b-catenin and p53/Rb pathways and promoter region methylation pattern) into a combined (COMBI) score. This molecular profile further improves the progression risk stratification identifying a group of patients with a favorable prognosis [36]. When superiority of COMBI with respect to mGRAS score was tested by discriminating patients with the best clinical outcome (at least 24 months free of disease progression).

COMBI score showed a better prognostic performance, proven by superior specificity (58.6\% vs. 31.0\%) and accuracy (83.3\% vs. 74.5\%). Also, when evaluating the diseasefree survival (DFS) in a selected group of patients with ACC who were successfully operated (RO). In this subgroup, only COMBI score was able to identify a category of patients with an extremely longer DFS [36]. However, molecular profiling is not part of the European Guideline on ACC [32]. In addition, genomic studies lead to better understanding of tumor biology and hopefully could yield new insights to develop new therapies where current therapeutic options are limited, and available (chemo) therapies of limited effectiveness [37].

Over the last decade the understanding of adrenocortical tumorigenesis has improved and recently studies started focusing on epigenetic changes associated with adrenocortical tumors. Global DNA hypomethylation is a thoroughly studied example of an epigenetic alteration which is a hallmark of both benign and malignant tumors with unique methylation patterns [38]. Epigenetic alterations occur frequently in cancer cells and have the ability to mimic the effects of the latter $[14,39,40]$. Such epigenetic alterations are becoming increasingly accessible to analyze for an individual patient, and would be an interesting layer of additional molecular information to existing clinicopathological methods. Additionally, Feinberg (2018) suggested that differences in tumor types are related to the tissue of origin and often to the 
spectrum of mutations associated with that organ, whereas properties of the tumor heterogeneity and therapeutic resistance are epigenetic and are shared among tumor types. Therefore, understanding epigenetic regulation in cancer in general could provide valuable information needed to improve therapeutic strategies [41].

\section{Genome Wide DNA Methylation}

Rechache et al. [42] were the first to report a genome-wide DNA methylation profiling study in adrenocortical tumors: 19 normal, 48 benign, eight primary malignant and 12 metastatic malignant ones. They found, using the Infinium HumanMethylation450 BeadChips (Illumina, San Diego, CA, USA), that methylation patterns were distinctly different between normal, benign, primary malignant and metastatic tissue samples. Differentially methylated sites were found in both coding and noncoding regions of DNA.

Interestingly, analysis of methylation patterns of benign adrenocortical tumor samples by functional status (cortisol secreting, aldosterone secreting, and nonfunctioning) showed different methylation patterns. Aldosterone-secreting tumor samples compared with nonfunctioning samples showed mostly hypomethylated CpG sites $(75,3)$. There were only a small amount of differentially hypermethylated CpG sites between cortisol-secreting tumor samples compared with aldosterone-secreting tumor samples. No significant differences in the methylation pattern between cortisol secreting tumors and nonfunctioning tumor samples were found [42].This raises the question whether it is the methylation analysis that is unable to show difference between cortisol secreting tumors and nonfunctioning tumor samples or it is the clinical definition of 'cortisol secreting' and 'nonfunctioning'.

In cancer, hypomethylation usually occurs at repeated DNA sequences whereas hypermethylation predominantly involves CpG Islands [43]. This was also observed in ACC. Primary and metastatic ACC samples were globally hypomethylated compared to normal and benign samples. Hypermethylation in primary and metastatic ACC samples was predominantly seen in islands $[42,44]$. DNA methylation of the H19 promoter has been shown to be involved in the abnormal expression of both $\mathrm{H} 19$ and IGF2 genes in the single gene study by Gao et al. (2002) [45]. Rechache et al. (2012) found 52 genes to be hypermethylated and downregulated in ACC (Table 2). Furthermore, of the differentially methylated genes in primary ACC, compared with benign tissue samples, several CpG sites were differentially methylated including those associated with KCTD12, KRREL, SYNGR1, and NTNG2 and those in chromosome 11 p15 imprinted region including IGF2 and H19. Other sites were also in the IGF2 pathway, including IGF1R that IGF2 binds to and AKT1, a downstream signaling molecule in the cell survival pathway of IGF1R. TP53 and CTNBB1 both had hypomethylated sites, RARRES2 and SC16A9 had several hypermethylated sites in ACC tissue samples. 
Table 2. Whole genome methylation studies on adrenocortical carcinoma.

\begin{tabular}{|c|c|c|c|c|c|}
\hline Study & Country & Year & $\mathrm{N}$ & Population & $\begin{array}{l}19 \mathrm{NA} ; 47 \text { Benign; } 8 \text { Primary } \\
\text { malignant; } 12 \text { Metastatic } \\
\text { malignant adrenals. }\end{array}$ \\
\hline \multirow[t]{7}{*}[42]{} & USA & 2012 & 87 & Method & $\begin{array}{l}\text { Infinium HumanMethylation } 450 \\
\text { BeadChips (Illumina, San Diego, CA, } \\
\text { USA) }\end{array}$ \\
\hline & & & & Results & $\begin{array}{l}\text { ACC show unique methylation } \\
\text { patterns in which gene methylation } \\
\text { status may be an important regulator } \\
\text { of gene expression. }\end{array}$ \\
\hline & & & & Hypomethylated & TP53, $\beta$ catenin (CTNNB1) \\
\hline & & & & Hypermethylated & 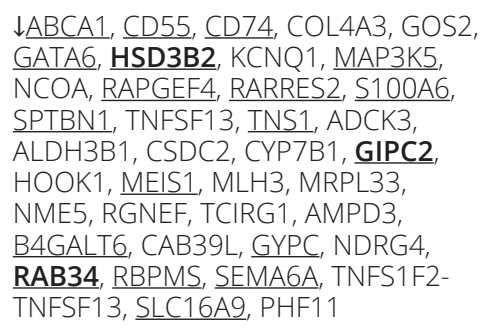 \\
\hline & & & & Diagnostic & $\begin{array}{l}\text { 'Determination of the methylation } \\
\text { difference in certain probe sites } \\
\text { in ACT may be a useful diagnostic } \\
\text { adjunct to histopathology for } \\
\text { localized primary ACC.' }\end{array}$ \\
\hline & & & & Prognostic & - \\
\hline & & & & Therapeutic & - \\
\hline \multirow[t]{8}{*}[46]{} & USA & 2012 & 48 & Population & $\begin{array}{l}6 \text { NA; } 27 \text { ACA (9 Nonfunctional,9 } \\
\text { Cortisol producing, } 9 \text { Aldosterone } \\
\text { producing); } 15 \text { ACC (9 Nonfunctional,6 } \\
\text { Cortisol producing) }\end{array}$ \\
\hline & & & & Method & $\begin{array}{l}\text { Infinium HumanMethylation27 } \\
\text { Beadchip (Illumina, San Diego, CA) }\end{array}$ \\
\hline & & & & Results & $\begin{array}{l}\text { CpG islands in the promoter regions are } \\
\text { significantly hypermethylated in ACC. }\end{array}$ \\
\hline & & & & Hypomethylated & \\
\hline & & & & Hypermethylated & $\begin{array}{l}\text { ZNF154, ALX4, } \downarrow \text { CDKN2A, GATA4, } \\
\text { SCGB3A1/HIN1, PYCARRD, HDAC10 } \\
\text { and DLEC1 }\end{array}$ \\
\hline & & & & Diagnostic & - \\
\hline & & & & Prognostic & - \\
\hline & & & & Therapeutic & $\begin{array}{l}\text { Treatment of ACC cell line H295R with } \\
5 \text {-aza-2'-deoxytide showed significant } \\
\text { restoration of gene expression of } \\
\text { CDKN2A, GATA4, DLEC1, HDAC10, } \\
\text { PYCARD and SCGB3A1/HIN1. }\end{array}$ \\
\hline
\end{tabular}




\begin{tabular}{|c|c|c|c|c|c|}
\hline Study & Country & Year & $\mathrm{N}$ & Population & $\begin{array}{l}19 \mathrm{NA} ; 47 \text { Benign; } 8 \text { Primary } \\
\text { malignant; } 12 \text { Metastatic } \\
\text { malignant adrenals. }\end{array}$ \\
\hline \multirow[t]{8}{*}{ [47] } & France & 2013 & 135 & Population & 84 ACA; 51 ACC \\
\hline & & & & Method & $\begin{array}{l}\text { Infinium HumanMethylation27 } \\
\text { Beadchip (Illumina, San Diego, CA) } \\
\text { MS-MLPA }\end{array}$ \\
\hline & & & & Results & $\begin{array}{l}\text { ACC samples can be categorized } \\
\text { according to CpG island methylator } \\
\text { phenotype. }\end{array}$ \\
\hline & & & & Hypomethylated & \\
\hline & & & & Hypermethylated & $\begin{array}{l}\downarrow \text { } 1 \text { 19, GSTM1, GSTP1, G0S2, GSTT1, } \\
\text { RAB34, GYPC, GIPC2, PLAGL1, LY6D, } \\
\text { PCOLCE, NDN, AMT, LGALS3BP, } \\
\text { APOC1, TM7SF2, PPAPDC3, PTPN7, } \\
\text { SCNN1A, HSD3B2, ACAA2, CTSZ, } \\
\text { PYGM, KRT8, NDRG2 }\end{array}$ \\
\hline & & & & Diagnostic & - \\
\hline & & & & Prognostic & $\begin{array}{l}\text { The global level of methylation in CpG } \\
\text { islands was associated with survival. } \\
\text { CIMP carcinomas were associated } \\
\text { with poorer prognosis. }\end{array}$ \\
\hline & & & & Therapeutic & - \\
\hline \multirow[t]{8}{*}[48]{} & France/ & 2014 & $81^{* \star}$ & Population & 51 ACC; 30 ACA \\
\hline & Europe & & & Method & $\begin{array}{l}\text { Infinium HumanMethylation27 } \\
\text { Beadchip (Illumina, San Diego, CA) }\end{array}$ \\
\hline & (ENSAT) & & & Results & $\begin{array}{l}\text { Confirmed CIMP in ACC. Tumor } \\
\text { clusters based on different genomic } \\
\text { approaches correlate. }\end{array}$ \\
\hline & & & & Hypomethylated & Nfs \\
\hline & & & & Hypermethylated & $\mathrm{Nfs}$ \\
\hline & & & & Diagnostic & - \\
\hline & & & & Prognostic & $\begin{array}{l}\text { Transcriptome clusters were strongly } \\
\text { correlated with DNA methylation } \\
\text { clusters. The C } 1 \text { A subgroup with poor } \\
\text { prognosis included almost all CIMP } \\
\text { and Mi3 tumors. C1B tumors with } \\
\text { good prognosis were generally non- } \\
\text { CIMP and belonged to the Mi1 or Mi2 } \\
\text { miRNA cluster. }\end{array}$ \\
\hline & & & & Therapeutic & - \\
\hline
\end{tabular}




\section{Study Country Year N Population 19 NA; 47 Benign; 8 Primary malignant; 12 Metastatic malignant adrenals.}

\begin{tabular}{llll}
\hline [49] USA 2015116 & $\begin{array}{l}\text { Population } \\
\text { Method }\end{array}$
\end{tabular}

Results

Hypomethylated

Hypermethylated

Diagnostic

Prognostic

Therapeutic
20 ACC; 75 Benign, 21NA

Infinium HumanMethylation 450 BeadChips (Illumina, San Diego, CA)

A cumulative comparison among gene methylation, copy number and miRNA profiling found that oncostatin M signaling, retinoic acid receptor activation (RXR) and PI3K/AKT and CDC42 signaling pathways were among the top pathways altered in ACC.

TIPARP, RAPGEF4, RAB34, PPTC7, PDZRN3, OBSL1, NCEH1, MTMR6, METTL7A, LONRF2, LIMCH1, KLF9, KIAA1024, JAK1, ITGAV, ITGA2,

HSD3B2, HLA-DPB1, DDC2, FOSL2, FGF12, FAMI198B, CYP1B1, CLU, CD59, CD55, C1QB, B4GALT6, IL13RA2, CDK1, ZMIZ1, TNS1, TBC1D4, SPTBN1, SLC16A9, SKAP2, SEMA6A, S100A6, RBPMS, RARRES2, RAB8B, PTPRG, PPP1R14A, NCOA7, MEIS1, MAP3K5, KCTD12, IL6ST, HTR2B, HOXA5, GIPC2, GATA6, GOS2, FSTL1, FMNL2, DDAH1, CD9, CD74, CD14, C3, C1RL, BNIP3L, AS3MT, APOC1, ABCA1, LPPR1, C9orf84

Treatment of the ACC cell line, H295R, with decitabine (a global methylation inhibitor) increased the gene expression of CYP1B1 dramatically. It was found that oncostatin M inhibits ACC cell proliferation. Oncostatin M could inhibit ACC cell growth 


\begin{tabular}{lll}
\hline Study Country Year N $\quad$ Population & $\begin{array}{l}19 \mathrm{NA} ; 47 \text { Benign; } 8 \text { Primary } \\
\text { malignant; } 12 \text { Metastatic } \\
\end{array}$ \\
& malignant adrenals.
\end{tabular}

[44] USA $201624 \quad$ Population

Method

Results

Hypomethylated

Hypermethylated

Diagnostic

Prognostic

Therapeutic

[17] USA $201679 * \star *$ Population

Method
Results
Hypomethylated
Hypermethylated

Diagnostic

Prognostic

Therapeutic
18 ACC (17 adrenal carcinomas, 1 liver metastasis); 6 NA

Infinium HumanMethylation 450 BeadChips (Illumina, San Diego, CA)

It was demonstrated that ACC are globally hypomethylated compared to normal adrenal tissue. Hypomethylation was most frequent in 'open seas' and hypermethylation mostly in CpG islands. Epigenetic modulation of genes involved in TP53 stability and function, WNT signaling, and tumor suppressor genes were found.

TMEM132D, ADCY2

i.a. EPHX3, MEIS, CCDC8, TBX3, PAX8, DUSP7, DYRK2, RBM5, SETD7, NDRG1, UBE2D1$$
-
$$

91 adrenal tumors: 84 usual type, 4 oncocytic, 2 sarcomatoid and 1 myxoid variant.

Infinium HumanMethylation 450 BeadChips (Illumina, San Diego, CA)

Identified three Coc subtypes

Nfs

\section{CDKN2A; NfS}

A methylation signature consisting of 68 probes robustly classified their cohort into three ACC survival groups with 92,4\% accuracy.

Coc analysis showed that molecular data can determine outcome with high significance.

\footnotetext{
Underlined results show overlap between two studies/Genes in bold were found to be hypermethylated in multiple studies. $\downarrow$ Under expressed genes. Adrenocortical Adenoma (ACA); Adrenocortical Carcinoma (ACC); Adrenocortical Tumors (ACT); CpG island promoter methylation (CIMP); Clusters of Cluster (Coc); European Network for the study of Adrenal Tumors (ENSAT); Normal adrenal (NA); Not further specified (nfs) ** [48] studied a total of 130 ACCS: 53 ACCs in their discovery cohort and 77 ACCs in their validation cohort. Only 51 samples from the discovery cohort were analyzed for DNA methylation profiling. *** [17] only analyzed 79 samples for DNA methylation profiling.
} 
Genetic studies have found IGF2 overexpression and CDKN1C and H19 downregulation in $90 \%$ of ACC cases $[33,50]$. In pediatric ACC, IGF1R overexpression is associated with a worse prognosis. Genetic mutations of the $\beta$-catenin gene are common in preferentially non-functioning adenomas and in ACC $[15,51]$.

Fonseca et al. [46] also analyzed the genome-wide methylation pattern of normal, benign and malignant adrenocortical tumors (Table 2). When comparing benign versus malignant ACT's, they found that $C p G$ islands were identified as significantly hypermethylated in ACC.

Primarily, genes involved in regulation of apoptosis, transcriptional, and cell cycle control showed significant and frequent hypermethylation. Only six genes known to be involved in the pathogenesis of other malignancies were further analyzed at mRNA level.

Expression of the significantly hypermethylated genes CDKN2A, GATA4, DLEC1, HDAC10, PYCARD, and SCB3A1/HIN1 was reduced in both ACAs and ACCS compared to normal adrenal tissue. When treating the H295R ACC cell line in vitro with a demethylating agent (5-aza-2'-deoxycytidine), expression of all hypermethylated genes increased.

This was observed earlier in the study by Gao et al. in which H19, hypermethylated expression increased after treating the H295R ACC cell line with a demethylating agent [45]. These results suggest that epigenetic alterations could be reversible and a potential therapeutic target [49].

\section{Candidate Gene Approach (Table 3)}

Since IGF2 is the most frequently overexpressed gene in ACC it is not surprising that single gene studies have focused on IGF2. Both Nielsen and Creemers studied differentially methylated regions patterns of IGF2. Three differentially methylated regions (DMRS) are involved in the regulation of IGF2 expression. DMRO, DMR2 and the imprinting control region (ICR). IGF2 DMRO and DMR2 are located between exons 2 and 3 and exons 8 and 9 respectively. 
Table 3. Single gene methylation studies on adrenocortical carcinoma.

\begin{tabular}{|c|c|c|c|c|c|}
\hline Study & Country & Year & $\mathrm{N}$ & Population & $\begin{array}{l}16 \text { NA; } 10 \text { ACC ( } 2 \text { Virilizing, } \\
2 \text { Nonfunctional, } 6 \text { Cushing's); } \\
16 \text { ACA ( } 2 \text { Virilizing, } 5 \text { Cushing's, } \\
5 \text { Conn's) }\end{array}$ \\
\hline \multirow[t]{8}{*}{ [45] } & Finland & 2002 & 46 & Gene & H19 \\
\hline & & & & Method & Bisulfite-PCR \\
\hline & & & & Results & $\begin{array}{l}\text { CpG sites in the H19 promoter } \\
\text { are hypermethylated in ACC. IGFII } \\
\text { is over expressed (methylation of } \\
\text { IGFII not analyzed) }\end{array}$ \\
\hline & & & & Hypermethylated & $\mathrm{H} 19$ \\
\hline & & & & Diagnostic & - \\
\hline & & & & Prognostic & - \\
\hline & & & & Therapeutic & $\begin{array}{l}\text { ACC cell line } \mathrm{NClH} 295 \mathrm{R} \text { was treated } \\
\text { with Azad, a demethylating agent. } \\
\text { It induced an increase in the H19 } \\
\text { RNA content. }\end{array}$ \\
\hline & & & & Population & $7 \mathrm{ACC} ; 8 \mathrm{ACA} ; 6 \mathrm{NA}$ \\
\hline \multirow[t]{8}{*}{ [52] } & USA & 2013 & 21 & Gene & RASSF1 \\
\hline & & & & Method & Epitect methyl II PCR \\
\hline & & & & Results & $\begin{array}{l}\text { There is a potential } \\
\text { oncosuppressor role for RASFF1 in } \\
\text { adrenocortical carcinogenesis. }\end{array}$ \\
\hline & & & & Hypermethylated & RASSF1 \\
\hline & & & & Diagnostic & - \\
\hline & & & & Prognostic & $\begin{array}{l}\text { All ACC showed reduced } \\
\text { expression of RASSf1A, irrespective } \\
\text { of their clinical characteristics or } \\
\text { malignant stages. }\end{array}$ \\
\hline & & & & Therapeutic & - \\
\hline & & & & Population & $\begin{array}{l}39 \text { ACA (16 Nonfunctional, } 16 \\
\text { Aldosterone producing, Cortisol } \\
\text { producing); } 3 \text { ACC; } 23 \mathrm{NA}\end{array}$ \\
\hline \multirow[t]{6}{*}{ [53] } & Japan & 2014 & 65 & Gene & Wif-1 \\
\hline & & & & Method & MSP, USP \& Bisulfite-PCR \\
\hline & & & & Results & $\begin{array}{l}57,1 \% \text { of the adrenal tumours } \\
\text { were found to be positive for } \\
\text { Wif- } 1 \text { methylation. No sub analysis } \\
\text { specific for ACC. }\end{array}$ \\
\hline & & & & Hypermethylated & Wif-1 \\
\hline & & & & Diagnostic & - \\
\hline & & & & Prognostic & - \\
\hline
\end{tabular}




\begin{tabular}{|c|c|c|c|c|c|}
\hline Study & Country & Year & $\mathrm{N}$ & Population & $\begin{array}{l}16 \text { NA; } 10 \text { ACC ( } 2 \text { Virilizing, } \\
2 \text { Nonfunctional, } 6 \text { Cushing's); } \\
16 \text { ACA ( } 2 \text { Virilizing, } 5 \text { Cushing's, } \\
5 \text { Conn's) }\end{array}$ \\
\hline & & & & Therapeutic & - \\
\hline & & & & Population & $3 \mathrm{NA} ; 19 \mathrm{ACC}$ \\
\hline \multirow[t]{8}{*}{ [54] } & Netherlands & 2014 & 22 & Gene & INHA \\
\hline & & & & Method & Bisulfite-PCR \\
\hline & & & & Results & $\begin{array}{l}\text { A subset of ACCs has an increased } \\
\text { methylation ratio of several CpGs in } \\
\text { the INHA promoter. }\end{array}$ \\
\hline & & & & Hypermethylated & INHA \\
\hline & & & & Diagnostic & \\
\hline & & & & Prognostic & $\begin{array}{l}\text { No association with van Slooten } \\
\text { index or ENSAT stage. }\end{array}$ \\
\hline & & & & Therapeutic & - \\
\hline & & & & Population & $\begin{array}{l}12 \text { Conn's; } 10 \text { Pheochromocytoma; } \\
20 \text { ACA, } 20 \text { ACC }\end{array}$ \\
\hline \multirow[t]{9}{*}{ [55] } & France & 2015 & 62 & Gene & IGF2 \\
\hline & & & & Method & Pyro-sequencing Bisulfite-PCR \\
\hline & & & & Results & $\begin{array}{l}\text { IGF2 overexpressed in } 85 \% \text { of } \\
\text { ACCs and } 100 \% \text { of PCC. Significant } \\
\text { decreased expression of H19 in } \\
\text { ACCs. }\end{array}$ \\
\hline & & & & & $\begin{array}{l}\text { 15/19 ACCs had somatic copy } \\
\text { number alterations at the IGF2/ } \\
\text { H19 locus, with } 6 / 15 \text { having an } \\
\text { extra copy of the allele. }\end{array}$ \\
\hline & & & & Hypomethylated & IGF2-DMR2 \\
\hline & & & & Hypermethylated & $\begin{array}{l}\text { H19-ICR (CTCF2, CTCF3, CTCF6); } 3 \\
\text { CPGS of DMRO }\end{array}$ \\
\hline & & & & Diagnostic & $\begin{array}{l}3 \text { CPGs of DRM0 correlated } \\
\text { positively with the Weiss score. }\end{array}$ \\
\hline & & & & ? & $\begin{array}{l}\text { Expression levels of IGF2 did not } \\
\text { correlate with clinical parameters } \\
\text { such as presence of metastases or } \\
\text { TNM stage. }\end{array}$ \\
\hline & & & & Prognostic & $\begin{array}{l}\text { The presence of more paternal } \\
\text { alleles than maternal alleles was } \\
\text { significantly associated with the } \\
\text { presence of metastases. }\end{array}$ \\
\hline
\end{tabular}




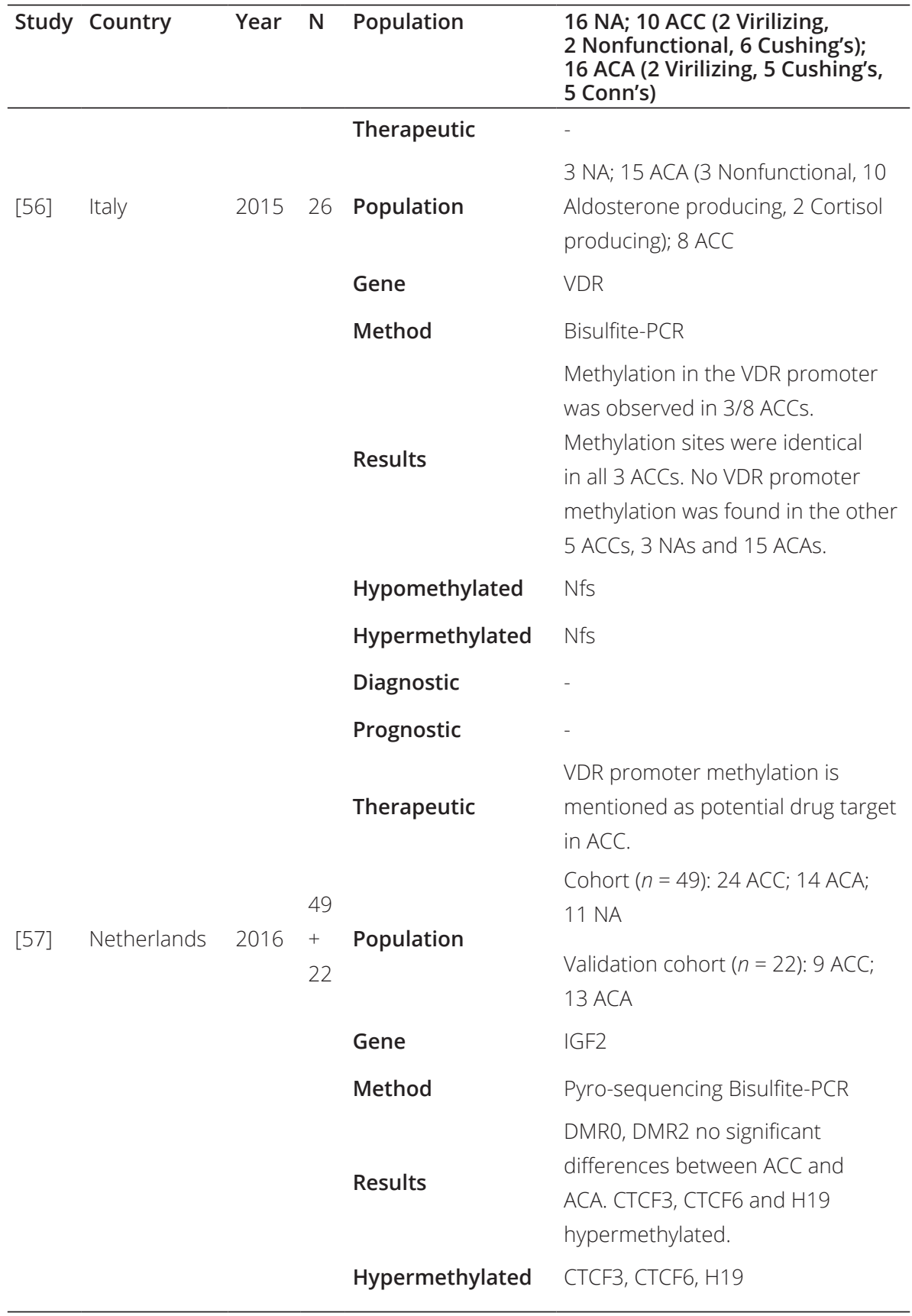




\begin{tabular}{cll}
\hline Study Country $\quad$ Year N Population & $\begin{array}{l}\text { 16 NA; } 10 \text { ACC (2 Virilizing, } \\
\text { 2 Nonfunctional, 6 Cushing's); } \\
\text { 16 ACA (2 Virilizing, 5 Cushing's, } \\
\text { 5 Conn's) }\end{array}$ \\
\hline Diagnostic & $\begin{array}{l}\text { IGF2 expression, DMR2, CTCF3 and } \\
\text { H19 showed a significant predictive } \\
\text { value for the diagnosis of ACC. }\end{array}$ \\
& - \\
& Treatment of three human ACC \\
& cell lines (H295R, HAC15 and \\
& SW13) with the demethylating drug \\
& AZA significantly decreased IGF2 \\
& expression and increased H19 \\
& expression. \\
&
\end{tabular}

Adrenocortical carcinoma (ACC); Differentially methylated regions (DMR); Imprinting Control Region (ICR); Methylation-specific PCR (MSP); Normal adrenals (NA) Pheochromocytoma (PCC); Not further specified (Nfs); Unmethylation-specific PCR (USP).

The H19 and IGF2 genes are separated from each other by the ICR. Nielsen found that $85 \%$ of the ACCs showed IGF2 overexpression and H19 down regulation, but did not found a correlation with clinical parameters such as the presence of metastases or TNM stage. The H19 DMR is located upstream of the H19 transcription start site. It harbors seven binding sites for the methylation-sensitive insulator CCCTC-binding factor (CTCF).

Methylation status of the ICR is of direct influence on CTCF binding. CTCF only binds unmethylated ICR on the maternal chromosome. Upon binding, CTCF prevents communication between the proximal H19 enhancer and the IGF2 promoter, therefore keeping IGF2 inactivated. CTCF cannot bind the paternal chromosome because the ICR is methylated. The enhancer is able to active IGF2 transcription from the paternal chromosome. CTCF serves as a position-dependent insulator element to block inappropriate enhancer signals and protect against forged gene activation [58]. Creemers et al. (2016) found a significant change in methylation in CTCF3 and CTCF6 between ACC and ACA, where methylation in ACCs was higher [57]. Also, H19 and IGF2 showed significant hypermethylation in ACC [57].

Recently an increased interest has been raised for the relation between vitamin $D$ and the adrenal gland [59]. Pilon et al. (2015) found methylation of the promoter of the vitamin D receptor and a reduced expression of the vitamin D receptor in ACC [56]. 
Earlier, inhibin alpha-subunit (INHA) was found to have a tumor suppressive role in adrenocortical tumorigenesis. In INHA knockout mice, 99\% developed steroidsecreting ACCs after gonadectomy [60,61]. Hofland et al. (2014) investigated the methylation and expression of Inhibin a-subunit (encoded by INHA) in adrenal tumors. They found a significant difference in methylation of the INHA promotor between normal adrenals and ACCS. However, the promoter methylation in the ACC samples was not associated with tumor characteristics or ENSAT stage.

\section{CIMP}

It was in a study for colorectal cancer that the CpG island promoter methylation, or CIMP, was first discovered [62]. The term CIMP is controversial [63] since there is actually no universal standard or consensus with respect to defining CIMP. It is used to describe the increased prevalence of CpG island promoter methylation. So far this phenomenon has been described in multiple types of cancer: bladder, breast, endometrial, gastric, glioblastoma, hepatocellular, lung, ovarian, pancreatic, prostate, and renal cell cancers as well as in leukemia, melanoma and neuroblastoma. In 2012 Barreau et al. [47] analyzed CIMP in adrenocortical cancer. Tumor material of 135 patients with adrenal tumors was collected and clinical outcome was registered. ACCs were globally more hypermethylated than ACAs at the CpG islands in the promoter regions.

ACCs were clustered in two groups with different methylation levels. The first group carcinomas was slightly hypermethylated compared with adenomas, the second group was hypermethylated compared with both the adenomas and the carcinomas from the first group. The second group was again subdivided according to methylation level: a CIMP-high subgroup and a CIMP-low subgroup. The level of methylation was associated with survival and CIMP carcinomas show a worse prognosis compared to non-CIMP tumors.

Gene expression levels were increasingly down-regulated when comparing non-CIMP, CIMP-low and CIMP high carcinomas. These data suggest that differential methylation in the CpG promoter regions could be of clinical importance since they provide a classification based on methylation as a marker for prognosis in patients with adrenal tumors.

Assié et al. (2014) [48] analyzed a cohort of 47 ACCS and an independent validation cohort of 77 ACCs, recruited from the European Network for the Study of Adrenal Tumors centers. Four DNA methylation-based tumor clusters were found. Two clusters corresponded to the CIMP-high and CIMP-low as described by Barreau et al. [47] associated with poor prognosis. Two other groups were categorized non-CIMP of which one showed widespread hypomethylation of CpG sites located outside CpG islands. 
miRNA expression was assayed and showed MIR483 to be overexpressed in ACC. MIR483 is located on the IGF2 locus, known to be involved in ACC. Based on miRNA expression levels Assié et al. (2014) identified three stable tumor clusters, Mi1-Mi3, with Mi1 having a significantly better overall survival rate then Mi2 and Mi3. Clusters were also established for mRNA expression in which two profiles were confirmed [34] to correlate strongly with survival, the aggressive C1A and indolent C1B. More importantly, a substantial overlap was found between the different omics of classifications. C1A (gene expression) with poor prognosis include almost all CIMP (DNA methylation) and Mi3 (miRNA expression) tumors. C1B tumors with a good prognosis were generally non-CIMP and belonged to the Mi1 or Mi2 mRNA clusters.

The Cancer Genome Atlas (TCGA), an unique landmark cancer genomics program, began in 2006 and since then molecularly characterized over 20,000 primary cancer and matched normal samples spanning 33 cancer types (PubMed searched on 1 April 2020: 8422 citations of TCGA \{search: "tcga"[Text Word]\}. In 2016 Zheng et al. [17] analyzed 91 histologically confirmed adrenal tumors and matched it with tissue from a global cohort including 84 usual type, four oncoytic, two sarcomatoid, and one myxoid variant. Their pan-genomic approach yielded four mRNA-expression groups, six microRNA-expression groups, three copy-number groups, three proteinexpression groups and three DNA-methylation groups. They integrated these ACC subsets through a cluster of clusters ( $\mathrm{CoC}$ ) analyses, resulting in three $\mathrm{CoC}$ subtypes, recognizing the fact that implementing four parallel profiling platforms poses a clinical challenge. The three methylation subtypes found (CIMP- low, intermediate and high) rendered discriminative representations of each CoC group, and classified the cohort into three ACC survival groups with $92.4 \%$ accuracy and were validated with an independent cohort.

Furthermore, it was shown that collectively the genes altered most frequently by somatic mutations, DNA copy-number alterations and epigenetic silencing were TP53 (21\%), ZNRF3 (19\%), CDKN2A (15\%), CTNNB1 (16\%), TERT (14\%) and PRKAR1A (11\%) [17].

\section{Histone Modification}

Histone modification is involved in the regulation of chromatin and gene expression. The best studied modifications are the acetylation and methylation of histones. Acetylation and deacetylation of histones is performed by histone modifying proteins such as histone acetyltransferases (HATs) and histone deacetylases (HDACS). At the transcriptional level, histone methylation is defined as the transfer of one, two, or three methyl groups from S-adenosyl-L-methionine to lysine or arginine residues of histone proteins by histone methyltransferases (HMTs). HMTs regulate DNA methylation through chromatin-dependent transcriptional repression or activation $[64,65]$. 
Drelon et al. performed a screen of histone methyltransferases, demethylases and associated factors in publicly available transcriptome data from ACC patients [66]. They observed the histone methyltransferase EZH2 to be overexpressed in ACC. High EZH2 expression levels, a result of deregulated P53/RB/E2f pathway, were associated increased cell proliferation/aggressive tumor behavior and poor prognosis in their study.

Zheng et al. reported that $22 \%$ of their analyzed samples had dysregulated mRNA expression levels of histone modification genes MLL, MLL2, and MLL4 and chromatin remodeling genes ATRX and DAXX [17]. Interestingly, seven percent of ACC cases have mutation in gene MEN1. MEN1 encodes the tumour suppressor, menin, which has been reported to interact with HMTS MLL, MLL2 [17,48].

\section{Epigenetics and ACC Treatment}

Genetic studies aimed at targeting biological pathways have not yet resulted in a significant breakthrough regarding therapeutic options. Inhibitors of both /G2/IGF1R and the mTOR pathways cause inhibition of cell proliferation of human ACC cell lines in vitro, and of growth of tumor xenografts in vivo $[67,68]$. A dual inhibitor of both /GF$1 R$ and $I R$, linsitinib (OSI-906), was studied for the first time in humans in an open-label phase I study of 79 patients with advanced solid tumors, of which 15 patients had ACC. Although efficacy was not the primary end point of the study, two patients with ACC had partial responses [69]. Therefore, linsitinib versus placebo was studied in a double-blind placebo-controlled phase III trial. Unfortunately, linsitinib failed to show a difference in median overall survival (OS) or progression free survival (PFS) [70].

Also, everolimus, an mTOR inhibitor, showed no clinically meaningful response in patients with stage IV ACC [71]. Other attempts targeting VEGF and EGFR have also met with modest success $[72,73]$. Studies suggest that DNA methylation, in addition to genetic modifications causes altered patterns of gene expression resulting in tumorigenesis and harvest potential therapeutic markers [12,74,75].

A role for temozolomide (TMZ), a cytotoxic and antiproliferative agent, has been proposed in the treatment for ACC, which is thought to act primarily by alkylation of specific sites on especially the $\mathrm{O}^{6}$ position of guanine, which mispairs with thymine during the next DNA replication cycle [76]. The methyl group in 06-methylguanine can be removed by the 06-methylguanine-DNA methyltransferase (MGMT) gene, which leads to and impaired efficacy of TMZ. Epigenetic marks regulating MGMT expression are used as a predictive marker for response to TMZ in glioblastoma patients, since epigenetic silencing of MGMT sensitizes glioblastoma cells to TMZ [77]. Creemers et al. showed that ACC cell lines appear to have a low MGMT promoter methylation and observed a trend toward a slightly higher MGMT methylation in the responsive primary ACC 
cultures [76]. Curiously, overexpression of PBK/TOPK promotes the chemotherapeutic resistance to TMZ in glioma [78]. Both low MGMT promoter methylations as well as PBK overexpression could contribute to the observed short-lived control rate and poor prognosis in a clinical study with TMZ in 28 ACC patients [79].

Epigenetic targeted drug reports are still limited to in vitro ACC cell line studies. The study by Gao et al. was mentioned earlier: they treated H295R with Azad (also known as 5-aza/decitabine), a demethylating agent (5-aza-2'-deoxycytidine), which led to a significant increase in the H19 RNA content [45]. Decitabine is a drug which is currently approved by the Food and Drug Administration (FDA) for the treatment of myelodysplastic syndromes. It reverses the DNA promoter methylation.

Suh et al. also tested decitabine NCl-H295R cells. They observed a significant decrease in ACC cell proliferation by 39\% to 47\% at 5 days after treatment compared with control specimens $(p<$.001) [74]. Interestingly, decitabine has been shown to potentiate the cytotoxic effects of current chemotherapies, such as doxorubicin, cisplatin and etoposide, in neuroblastoma, suggesting that a combination of 5-aza with standard therapies could lead to more effective treatment [80].

Vorinostat was one of the first drugs to be approved that influence post-translational modification of histone proteins. Demeure et al. tested vorinostat in an ACC cell line which resulted growth inhibition. No studies on HMT inhibition were found. EHZ2 being overexpressed in ACC, HMT inhibition could be a potential treatment strategy in ACC.

Further research is required to determine the role of epigenetic targeted drugs in the treatment of ACC and overcoming drug resistance, where in other types of cancer epigenetic therapies are an emerging option for overcoming drug resistance [81].

Pan-genomic studies will initially contribute to the process of matching clinical/ molecular profiles of patients with ACC with specific therapeutic programs and the understanding of therapeutic failures in the past. As Grisanti et al. noted, the COC1 cluster identified by Zheng et al. is characterized by a low grade of aneuploidy, better survival outcome, and high expression of the IGF2 pathway $[17,82]$. It could be considered that patients falling in this category would likely be more responsive to antielGF2/IGF-1R compounds. Whereas, patients in the COC3 group characterized by mutations involving the cell cycle and DNA damage repair machinery would probably better respond to chemotherapy. Eventually the focus will shift to the understanding and identification of cancer dependencies based on functional genomic data and selection of priority drug candidates/drug repositioning in ACC.

Actually, Zheng et al. found 51 potentially actionable alterations in 22 ACCs, considering existing clinical trials and FDA-approved drugs for cancers [17]. Recently two excellent reviews have been published on this issue $[75,83]$ which we encourage interested readers to consult. 


\section{Discussion}

In cancer in general, there has been growing evidence to suggest that DNA methylation in addition to direct genetic modification causes altered gene expression resulting in tumorigenesis. Epigenetic analysis in adrenocortical tumors so far has been a significant addition to the understanding of molecular events involved in adrenocortical carcinogenesis. DNA methylation- based tumor clusters show overlap with other omics classifications. Clustering on epigenetic level allows differentiation between benign and malignant tumors and could be a significant addition to current histological parameters. Moreover, it might serve as an addition to current ENSAT staging in order to estimate prognosis and tumor aggressiveness. Currently no biomarker is included in the European Society of Endocrinology Clinical Practice Guidelines on ACC, but it is stated in the guideline that patients' participation in registries and the collection of biological material as part of structured research programs aimed at defining biomarkers of diagnosis, prognosis and treatment response is encouraged [32].

An interesting observation in the studies discussed above is the comparison between genetic and transcriptome-based studies (Figure 2). IGF2 overexpression and structural abnormalities of 11 p15 are present in up to $90 \%$ of cases of human ACC. IGF2 expression is mediated by the insulin-like growth factor 1 receptor (IGF1R) which is also overexpressed in ACC. These genes have altered DNA methylation expression patterns in ACC. Zheng et al. found that $69 \%$ of tumours had at least one alteration of potential driver genes when combining somatic mutations, copy-number alterations, and epigenetic modification [17]. 


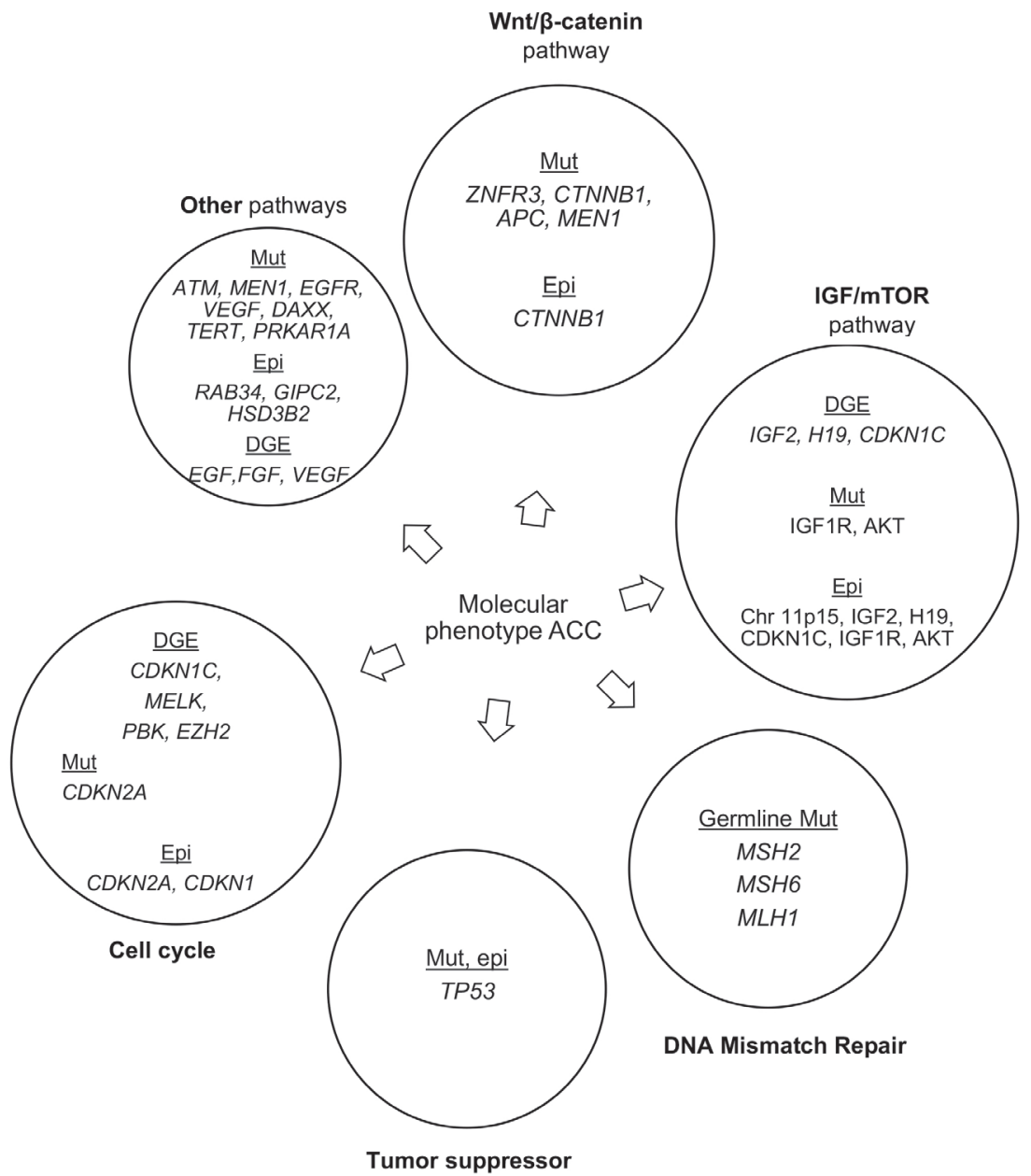

Figure 2. Frequently altered pathways in adrenocortical carcinoma as discussed in this review. Mut: mutations; DGE: differential gene expression; Epi: epigenetic modifications.

Further research is needed to understand the implications of epigenetic changes in adrenal tumorigenesis. Comprehensive data on well powered series are needed. For an orphan disease like ACC, united multinational consortia studies have the best chance of providing well powered data.

Results of methylation associated gene expression levels in the articles discussed show heterogeneity (Table 2). For example, the 52 genes identified by Rechache et al. do not completely overlap with the top genes identified by Barreau et al. This discrepancy may be caused by methodological differences. Rechache et al. [42] used 
the 450 BeadChip, which provides a more comprehensive coverage of the genome with 17 times more CpG sites than the 27BeadChip that was used in the other studies. Nevertheless, when using Infinium 450k microarrays, data still is restricted to the particular genomic locations of the probes used in the array, which might not even necessarily capture the most relevant methylation sites. Koch et al. already advocated that a better understanding and more detailed analysis of the clinical relevance of the genomic location of DNA methylation is required to increase the number biomarkers that can be successfully implemented in patient care [84].

Also, it should be decided which platform of genomic analysis will be used in daily practice. Because, effectively, what is probably needed is a multi-omic molecular panel with the best selected biomarker predictors. 


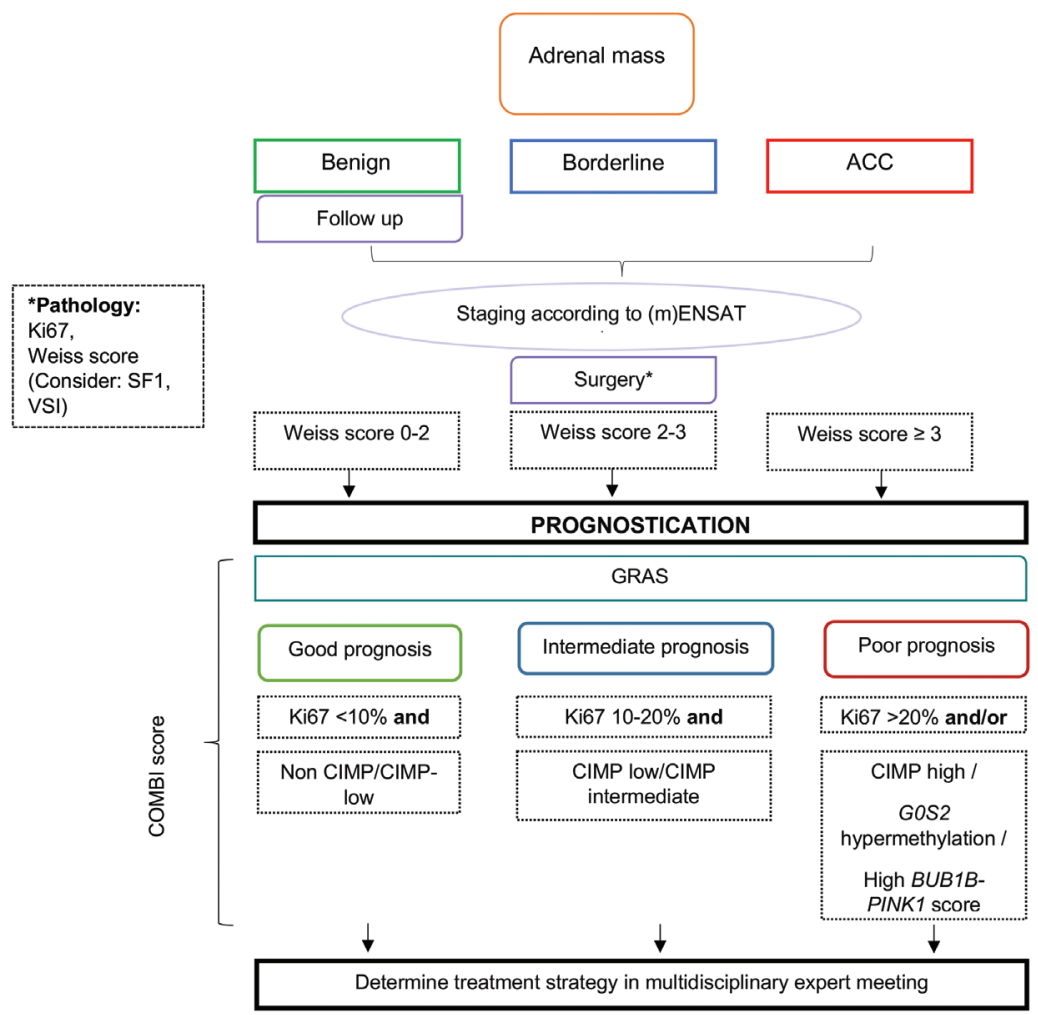

\begin{tabular}{|l|}
\hline RO: Active surveillance, consider \\
Mitotane (ADIUVO I) \\
Rx/R1: Mitotane + consider RT \\
$\mathrm{R}^{*}$ : \\
- Consider neo-adjuvant treatment \\
- Multi-omics guided clinical trial \\
\\
\hline
\end{tabular}

R0: Adjuvant Mitotane
Rx/R1: Mitotane + consider RT
$\mathrm{R}^{\star}$ :
- Consider neo-adjuvant treatment
- Multi-omics guided clinical trial

RO: Adjuvant Mitotane; consider chemotherapy (ADIUVO II):

Cisplatin + Mitotane (and, optional, Etoposide)

Rx/R1: Mitotane + RT, EDP

$\mathrm{R}^{*}$ :

- Consider debulking surgery

- Mitotane +/- local therapies

- Mitotane + EDP

- Multi-omics guided clinical trial

- Supportive care

Figure 3. Flowchart on the potential management of the adrenal mass with the implementation of genomic analysis. Adrenocortical Carcinoma (ACC); Etoposide doxorubicin cisplatin (EDP); (modified) European Network for the Study of Adrenal Tumors ((m)ENSAT); Grade, Resection status, Age, Symptoms (GRAS); Complete resection (R0); Unknown radicality (Rx); Microscopically irradical (R1); ACC not amendable to radical resection $\left(R^{*}\right)$; Radiotherapy (RT); Steroidogenic factor-1 (SF1); Van Slooten Index (VSI). 
DNA-methylation has proven to be replicable and able to provide accurate data on formalin-fixed or paraffin-embedded tumor samples $[85,86]$ but no data has been published comparing the available genomic cluster entities in their ability to correctly diagnose adrenal malignancy and to predict recurrence, progression free survival and overall survival. Currently have been opted: C1A/C1B cluster $[33,34,48]$ based on gene expressions, CIMP low/intermediate/high [17] cluster or CIMP low/high and non-CIMP [48] based on methylation profiles, CoC I-III as an integrated subset based on DNA copy number, DNA-methylation, mRNA-expression and miRNA-expression and Mi1-2 based on MiRNA expression. It is of importance to see how these clusters will perform when tested prospectively in a large cohort of adrenal tumors to validate these data and also establish the required cutoff values for the diagnosis of malignancy. Within these discriminating clusters, studies already are making an effort to identify markers representing these cluster sub-types. GOS2 hypermethylation was shown to be a hallmark of the CIMP-high cluster [87]. When validated GOS2 hypermethylation and the BUB1B-PINK1 score could be potential markers on a molecular panel for ACC $[34,87,88]$.

Next steps will include the prospective comparison of the pathologic classification (Ki67 and Weiss score) of adrenal tumors versus a genomic assay versus the combination of both in the process of accurately diagnosing adrenal tumors. Evidence is needed that molecular data can improve the current diagnostic tools and that it does not matter whether it is genetic or epigenetic data. Finally, the bold step needs to be made to test the predictive value of these classifications in clinical practice by choosing a treatment regimen (Figure 3) based on the ACC prognostic cluster. Creemers et al. already showed that including IGF2 methylation status to the pathology review could be supportive for the decision of adjuvant mitotane treatment [57]. Only after these steps, biomarkers could be officially implemented in the guidelines and acquire FDA approval.

Epigenetic changes may contribute to adrenocortical tumorigenesis by modulating size of the stem/progenitor population, altering phenotypic plasticity and enhancing sensitivity to subsequent mutations. ACC may develop in a multistep process. Therefore, it could be suggested that the level of DNA methylation is correlated with the risk of subsequent mutations, in which quantifying the influence of DNA methylation on gene expression remains difficult. Mutations in the Wnt/ $\beta$ catenin pathway have been shown to occur during progression $[16,89]$. Epigenetic and genetic mutations reflect alternate ways of inactivation during tumor progressions, i.e. a synergy between epigenetic and genetic alterations causing tumorigenesis, suggesting that combined inhibition of multiple affected pathways may hold the key to successful targeted therapy for ACC. 


\section{Conclusions}

Research on adrenocortical tumors has been dominated by gene expression profiling and by analysis of genetic disorders associated with the predisposition of these tumors. With epigenetic studies, we are entering a new and complex phase in the understanding of ACC tumorigenesis. Analyzing the relationship between alterations in different layers of gene regulation could yield interesting insights.

Finally, it will be challenging to not only use epigenetic analysis for diagnostic and prognostic purposes but also to keep investing in the development of new pharmacologic therapies and explore the potential of demethylating agents, because currently no significant therapeutic breakthrough is emerging. In the near future it will become interesting to see how the vast development of artificial intelligence, radiomics etc. will be of impact on diagnosis, prognosis and treatment of ACC. 


\section{References}

1. Abecassls, M.; McLoughlin, M.; Langer, B.; Kudlow, J. Serendipitous Adrenal Masses: Prevalence, Significance, and Management. The American journal of surgery 1985, 149, 783-788.

2. Glazer, H.S.; Weyman, P.J.; Sagel, S.S.; Levitt, R.G.; McClennan, B.L. Nonfunctioning Adrenal Masses: Incidental Discovery on Computed Tomography. AJR Am. J. Roentgenol. 1982, 139, 81-85.

3. Barzon, L.; Sonino, N.; Fallo, F.; Palu, G.; Boscaro, M. Prevalence and Natural History of Adrenal Incidentalomas. Eur. J. Endocrinol. 2003, 149, 273-285.

4. Herrera, M.F.; Grant, C.S.; van Heerden, J.A.; Sheedy, P.F.; Ilstrup, D.M. Incidentally Discovered Adrenal Tumors: An Institutional Perspective. Surgery 1991, 110, 1014-1021.

5. Bovio, S.; Cataldi, A.; Reimondo, G.; Sperone, P.; Novello, S.; Berruti, A.; Borasio, P.; Fava, C.; Dogliotti, L.; Scagliotti, G.V. et al. Prevalence of Adrenal Incidentaloma in a Contemporary Computerized Tomography Series. J. Endocrinol. Invest. 2006, 29, 298-302.

6. Fassnacht, M.; Arlt, W.; Bancos, I.; Dralle, H.; Newell-Price, J.; Sahdev, A.; Tabarin, A.; Terzolo, M.; Tsagarakis, S.; Dekkers, O.M. Management of Adrenal Incidentalomas: European Society of Endocrinology Clinical Practice Guideline in Collaboration with the European Network for the Study of Adrenal Tumors. Eur. J. Endocrinol. 2016, 175, G1-G34.

7. Ribeiro, R.C.; Figueiredo, B. Childhood Adrenocortical Tumours. Eur. J. Cancer 2004, 40, 1117-1126.

8. Bernstein, L.; Gurney, J.G. Carcinomas and Other Malignant Epithelial Neoplasms. Cancer and survival among children and adolescents: United States SEER program 1975, 1995, 139-147.

9. Else, T.; Kim, A.C.; Sabolch, A.; Raymond, V.M.; Kandathil, A.; Caoili, E.M.; Jolly, S.; Miller, B.S.; Giordano, T.J.; Hammer, G.D. Adrenocortical Carcinoma. Endocr. Rev. 2013, 35, 282-326.

10. Brennan, M.F. Adrenocortical Carcinoma. CA Cancer. J. Clin. 1987, 37, 348-365.

11. Rodriguez-Galindo, C.; Figueiredo, B.C.; Zambetti, G.P.; Ribeiro, R.C. Biology, Clinical Characteristics, and Management of Adrenocortical Tumors in Children. Pediatric blood \& cancer 2005, 45, 265-273.

12. Lerario, A.M.; Moraitis, A.; Hammer, G.D. Genetics and Epigenetics of Adrenocortical Tumors. Mol. Cell. Endocrinol. 2014, 386, 67-84.

13. Herrmann, L.J.M.; Heinze, B.; Fassnacht, M.; Willenberg, H.S.; Quinkler, M.; Reisch, N.; Zink, M.; Allolio, B.; Hahner, S. TP53 Germline Mutations in Adult Patients with Adrenocortical Carcinoma. Journal of Clinical Endocrinology \& Metabolism 2011.

14. Bielinska, M.; Parviainen, H.; Kiiveri, S.; Heikinheimo, M.; Wilson, D.B. Review Paper: Origin and Molecular Pathology of Adrenocortical Neoplasms. Vet. Pathol. 2009, 46, 194-210.

15. Gaujoux, S.; Grabar, S.; Fassnacht, M.; Ragazzon, B.; Launay, P.; Libe, R.; Chokri, I.; Audebourg, A.; Royer, B.; Sbiera, S. et al. Beta-Catenin Activation is Associated with Specific Clinical and Pathologic Characteristics and a Poor Outcome in Adrenocortical Carcinoma. Clin. Cancer Res. 2011, 17, 328-336.

16. Tissier, F.; Cavard, C.; Groussin, L.; Perlemoine, K.; Fumey, G.; Hagnere, A.M.; Rene-Corail, F.; Jullian, E.; Gicquel, C.; Bertagna, X. et al. Mutations of Beta-Catenin in Adrenocortical Tumors: Activation of the Wnt Signaling Pathway is a Frequent Event in both Benign and Malignant Adrenocortical Tumors. Cancer Res. 2005, 65, 7622-7627.

17. Zheng, S.; Cherniack, A.D.; Dewal, N.; Moffitt, R.A.; Danilova, L.; Murray, B.A.; Lerario, A.M.; Else, T.; Knijnenburg, T.A.; Ciriello, G. Comprehensive Pan-Genomic Characterization of Adrenocortical Carcinoma. Cancer cell 2016, 29, 723-736.

18. Kiseljak-Vassiliades, K.; Zhang, Y.; Bagby, S.M.; Kar, A.; Pozdeyev, N.; Xu, M.; Gowan, K.; Sharma, V.; Raeburn, C.D.; Albuja-Cruz, M. Development of New Preclinical Models to Advance Adrenocortical Carcinoma Research. Endocr. Relat. Cancer 2018, 25, 437-451. 
19. Wang, T.; Rainey, W.E. Human Adrenocortical Carcinoma Cell Lines. Mol. Cell. Endocrinol. 2012, 351, 58-65.

20. Kiseljak-Vassiliades, K.; Zhang, Y.; Kar, A.; Razzaghi, R.; Xu, M.; Gowan, K.; Raeburn, C.D.; AlbujaCruz, M.; Jones, K.L.; Somerset, H. Elucidating the Role of the Maternal Embryonic Leucine Zipper Kinase in Adrenocortical Carcinoma. Endocrinology 2018, 159, 2532-2544.

21. Kar, A.; Zhang, Y.; Yacob, B.W.; Saeed, J.; Tompkins, K.D.; Bagby, S.M.; Pitts, T.M.; Somerset, H.; Leong, S.; Wierman, M.E. Targeting PDZ-Binding Kinase is Anti-Tumorigenic in Novel Preclinical Models of ACC. Endocr. Relat. Cancer 2019, 26, 765-778.

22. Fassnacht, M.; Johanssen, S.; Quinkler, M.; Bucsky, P.; Willenberg, H.S.; Beuschlein, F.; Terzolo, M.; Mueller, H.H.; Hahner, S.; Allolio, B. Limited Prognostic Value of the 2004 International Union Against Cancer Staging Classification for Adrenocortical Carcinoma: Proposal for a Revised TNM Classification. Cancer 2009, 115, 243-250.

23. Hermsen, I.G.; Gelderblom, H.; Kievit, J.; Romijn, J.A.; Haak, H.R. Extremely Long Survival in Six Patients Despite Recurrent and Metastatic Adrenal Carcinoma. Eur. J. Endocrinol. 2008, 158, 911-919.

24. Berruti, A.; Fassnacht, M.; Baudin, E.; Hammer, G.; Haak, H.; Leboulleux, S.; Skogseid, B.; Allolio, B.; Terzolo, M. Adjuvant Therapy in Patients with Adrenocortical Carcinoma: A Position of an International Panel. J. Clin. Oncol. 2010, 28, e401-2; author reply e403.

25. Papathomas, T.G.; Pucci, E.; Giordano, T.J.; Lu, H.; Duregon, E.; Volante, M.; Papotti, M.; Lloyd, R.V.; Tischler, A.S.; van Nederveen, F.H. et al. An International Ki67 Reproducibility Study in Adrenal Cortical Carcinoma. Am. J. Surg. Pathol. 2016, 40, 569-576.

26. Fassnacht, M.; Kroiss, M.; Allolio, B. Update in Adrenocortical Carcinoma. J. Clin. Endocrinol. Metab. 2013, 98, 4551-4564.

27. Beuschlein, F.; Weigel, J.; Saeger, W.; Kroiss, M.; Wild, V.; Daffara, F.; Libe, R.; Ardito, A.; Al Ghuzlan, A.; Quinkler, M. et al. Major Prognostic Role of Ki67 in Localized Adrenocortical Carcinoma After Complete Resection. J. Clin. Endocrinol. Metab. 2015, 100, 841-849.

28. Duregon, E.; Cappellesso, R.; Maffeis, V.; Zaggia, B.; Ventura, L.; Berruti, A.; Terzolo, M.; Fassina, A.; Volante, M.; Papotti, M. Validation of the Prognostic Role of the "Helsinki Score" in 225 Cases of Adrenocortical Carcinoma. Hum. Pathol. 2016.

29. Jouinot, A.; Assie, G.; Libe, R.; Fassnacht, M.; Papathomas, T.; Barreau, O.; De La Villeon, B.; Faillot, S.; Hamzaoui, N.; Neou, M. DNA Methylation is an Independent Prognostic Marker of Survival in Adrenocortical Cancer. The Journal of Clinical Endocrinology \& Metabolism 2017, 102, 923-932.

30. Libe, R.; Borget, I.; Ronchi, C.L.; Zaggia, B.; Kroiss, M.; Kerkhofs, T.; Bertherat, J.; Volante, M.; Quinkler, M.; Chabre, O. et al. Prognostic Factors in Stage III-IV Adrenocortical Carcinomas (ACC): An European Network for the Study of Adrenal Tumor (ENSAT) Study. Ann. Oncol. 2015, 26, 2119-2125.

31. Ronchi, C.L.; Sbiera, S.; Leich, E.; Tissier, F.; Steinhauer, S.; Deutschbein, T.; Fassnacht, M.; Allolio, B. Low SGK1 Expression in Human Adrenocortical Tumors is Associated with ACTH-Independent Glucocorticoid Secretion and Poor Prognosis. The Journal of Clinical Endocrinology \& Metabolism 2012, 97, E2251-E2260.

32. Fassnacht, M.; Dekkers, O.; Else, T.; Baudin, E.; Berruti, A.; De Krijger, R.R.; Haak, H.; Mihai, R.; Assie, G.; Terzolo, M. European Society of Endocrinology Clinical Practice Guidelines on the Management of Adrenocortical Carcinoma in Adults, in Collaboration with the European Network for the Study of Adrenal Tumors. European journal of endocrinology 2018, 1.

33. Giordano, T.J.; Kuick, R.; Else, T.; Gauger, P.G.; Vinco, M.; Bauersfeld, J.; Sanders, D.; Thomas, D.G.; Doherty, G.; Hammer, G. Molecular Classification and Prognostication of Adrenocortical Tumors by Transcriptome Profiling. Clin. Cancer Res. 2009, 15, 668-676. 
34. De Reynies, A.; Assie, G.; Rickman, D.S.; Tissier, F.; Groussin, L.; Rene-Corail, F.; Dousset, B.; Bertagna, X.; Clauser, E.; Bertherat, J. Gene Expression Profiling Reveals a New Classification of Adrenocortical Tumors and Identifies Molecular Predictors of Malignancy and Survival. J. Clin. Oncol. 2009, 27, 1108-1115.

35. Giordano, T.J. Adrenocortical Tumors: An Integrated Clinical, Pathologic, and Molecular Approach at the University of Michigan. Arch. Pathol. Lab. Med. 2010, 134, 1440-1443.

36. Lippert, J.; Appenzeller, S.; Liang, R.; Sbiera, S.; Kircher, S.; Altieri, B.; Nanda, I.; Weigand, I.; Gehrig, A.; Steinhauer, S. Targeted Molecular Analysis in Adrenocortical Carcinomas: A Strategy Toward Improved Personalized Prognostication. The Journal of Clinical Endocrinology \& Metabolism 2018, 103, 4511-4523.

37. Fassnacht, M.; Libe, R.; Kroiss, M.; Allolio, B. Adrenocortical Carcinoma: A Clinician's Update. Nat. Rev. Endocrinol. 2011, 7, 323-335.

38. Vucic, E.A.; Brown, C.J.; Lam, W.L. Epigenetics of Cancer Progression. Pharmacogenomics 2008, 9, 215-234.

39. Feinberg, A.P. Phenotypic Plasticity and the Epigenetics of Human Disease. Nature 2007, 447, 433-440.

40. Feinberg, A.P.; Ohlsson, R.; Henikoff, S. The Epigenetic Progenitor Origin of Human Cancer. Nat. Rev. Genet. 2006, 7, 21-33.

41. Feinberg, A.P. The Key Role of Epigenetics in Human Disease Prevention and Mitigation. N. Engl. J. Med. 2018, 378, 1323-1334.

42. Rechache, N.S.; Wang, Y.; Stevenson, H.S.; Killian, J.K.; Edelman, D.C.; Merino, M.; Zhang, L.; Nilubol, N.; Stratakis, C.A.; Meltzer, P.S. et al. DNA Methylation Profiling Identifies Global Methylation Differences and Markers of Adrenocortical Tumors. J. Clin. Endocrinol. Metab. 2012, 97, E1004-13.

43. Irizarry, R.A.; Ladd-Acosta, C.; Wen, B.; Wu, Z.; Montano, C.; Onyango, P.; Cui, H.; Gabo, K.; Rongione, M.; Webster, M. et al. The Human Colon Cancer Methylome shows Similar Hypo- and Hypermethylation at Conserved Tissue-Specific CpG Island Shores. Nat. Genet. 2009, 41, 178-186.

44. Legendre, C.R.; Demeure, M.J.; Whitsett, T.G.; Gooden, G.C.; Bussey, K.J.; Jung, S.; Waibhav, T.; Kim, S.; Salhia, B. Pathway Implications of Aberrant Global Methylation in Adrenocortical Cancer. PLoS One 2016, 11, e0150629.

45. Gao, Z.H.; Suppola, S.; Liu, J.; Heikkila, P.; Janne, J.; Voutilainen, R. Association of H19 Promoter Methylation with the Expression of $\mathrm{H} 19$ and IGF-II Genes in Adrenocortical Tumors. J. Clin. Endocrinol. Metab. 2002, 87, 1170-1176.

46. Fonseca, A.L.; Kugelberg, J.; Starker, L.F.; Scholl, U.; Choi, M.; Hellman, P.; Akerstrom, G.; Westin, G.; Lifton, R.P.; Bjorklund, P. et al. Comprehensive DNA Methylation Analysis of Benign and Malignant Adrenocortical Tumors. Genes Chromosomes Cancer 2012, 51, 949-960.

47. Barreau, O.; Assie, G.; Wilmot-Roussel, H.; Ragazzon, B.; Baudry, C.; Perlemoine, K.; Rene-Corail, F.; Bertagna, X.; Dousset, B.; Hamzaoui, N. et al. Identification of a CpG Island Methylator Phenotype in Adrenocortical Carcinomas. J. Clin. Endocrinol. Metab. 2013, 98, E174-84.

48. Assie, G.; Letouze, E.; Fassnacht, M.; Jouinot, A.; Luscap, W.; Barreau, O.; Omeiri, H.; Rodriguez, S.; Perlemoine, K.; Rene-Corail, F. et al. Integrated Genomic Characterization of Adrenocortical Carcinoma. Nat. Genet. 2014, 46, 607-612.

49. Gara, S.K.; Wang, Y.; Patel, D.; Liu-Chittenden, Y.; Jain, M.; Boufraqech, M.; Zhang, L.; Meltzer, P.S.; Kebebew, E. Integrated Genome-Wide Analysis of Genomic Changes and Gene Regulation in Human Adrenocortical Tissue Samples. Nucleic Acids Res. 2015, 43, 9327-9339.

50. Giordano, T.J.; Thomas, D.G.; Kuick, R.; Lizyness, M.; Misek, D.E.; Smith, A.L.; Sanders, D.; Aljundi, R.T.; Gauger, P.G.; Thompson, N.W. et al. Distinct Transcriptional Profiles of Adrenocortical Tumors Uncovered by DNA Microarray Analysis. Am. J. Pathol. 2003, 162, 521-531. 
51. Hermsen, I.G.; Haak, H.R.; de Krijger, R.R.; Kerkhofs, T.M.; Feelders, R.A.; de Herder, W.W.; Wilmink, H.; Smit, J.W.; Gelderblom, H.; de Miranda, N.F. et al. Mutational Analyses of Epidermal Growth Factor Receptor and Downstream Pathways in Adrenocortical Carcinoma. Eur. J. Endocrinol. 2013, 169, 51-58.

52. Korah, R.; Healy, J.M.; Kunstman, J.W.; Fonseca, A.L.; Ameri, A.H.; Prasad, M.L.; Carling, T. Epigenetic Silencing of RASSF1A Deregulates Cytoskeleton and Promotes Malignant Behavior of Adrenocortical Carcinoma. Molecular cancer 2013, 12, 87.

53. Mitsui, Y.; Yasumoto, H.; Nagami, T.; Hiraki, M.; Arichi, N.; Ishikawa, N.; Araki, A.; Maruyama, R.; Tanaka, Y.; Dahiya, R. et al. Extracellular Activation of Wnt Signaling through Epigenetic Dysregulation of Wnt Inhibitory Factor-1 (Wif-1) is Associated with Pathogenesis of Adrenocortical Tumor. Oncotarget 2014, 5, 2198-2207.

54. Hofland, J.; Steenbergen, J.; Voorsluijs, J.M.; Verbiest, M.M.; de Krijger, R.R.; Hofland, L.J.; de Herder, W.W.; Uitterlinden, A.G.; Feelders, R.A.; de Jong, F.H. Inhibin Alpha-Subunit (INHA) Expression in Adrenocortical Cancer is Linked to Genetic and Epigenetic INHA Promoter Variation. PLoS One 2014, 9, e104944.

55. Nielsen, H.M.; How-Kit, A.; Guerin, C.; Castinetti, F.; Vollan, H.K.; De Micco, C.; Daunay, A.; Taieb, D.; Van Loo, P.; Besse, C. et al. Copy Number Variations Alter Methylation and Parallel IGF2 Overexpression in Adrenal Tumors. Endocr. Relat. Cancer 2015, 22, 953-967.

56. Pilon, C.; Rebellato, A.; Urbanet, R.; Guzzardo, V.; Cappellesso, R.; Sasano, H.; Fassina, A.; Fallo, F. Methylation Status of Vitamin D Receptor Gene Promoter in Benign and Malignant Adrenal Tumors. International journal of endocrinology 2015, 2015.

57. Creemers, S.; Van Koetsveld, P.; van Kemenade, F.; Papathomas, T.; Franssen, G.; Dogan, F.; Eekhoff, E.; van der Valk, P.; de Herder, W.; Janssen, J. Methylation of IGF2 Regulatory Regions to Diagnose Adrenocortical Carcinomas. Endocr. Relat. Cancer 2016, 23, 727-737.

58. Kim, S.; Yu, N.; Kaang, B. CTCF as a Multifunctional Protein in Genome Regulation and Gene Expression. Exp. Mol. Med. 2015, 47, e166-e166.

59. Muscogiuri, G.; Mitri, J.; Mathieu, C.; Badenhoop, K.; Tamer, G.; Orio, F.; Mezza, T.; Vieth, R.; Colao, A.; Pittas, A. Vitamin D as a Potential Contributor in Endocrine Health and Disease. Eur. J. Endocrinol. 2014, 171, R101-R110.

60. Matzuk, M.M.; Finegold, M.J.; Su, J.; Hsueh, A.; Bradley, A. Alpha-Inhibin is a Tumour-Suppressor Gene with Gonadal Specificity in Mice. Nature 1992, 360, 313.

61. Matzuk, M.M.; Finegold, M.J.; Mather, J.P.; Krummen, L.; Lu, H.; Bradley, A. Development of Cancer Cachexia-Like Syndrome and Adrenal Tumors in Inhibin-Deficient Mice. Proc. Natl. Acad. Sci. U. S. A. 1994, 91, 8817-8821.

62. Toyota, M.; Ahuja, N.; Ohe-Toyota, M.; Herman, J.G.; Baylin, S.B.; Issa, J.P. CpG Island Methylator Phenotype in Colorectal Cancer. Proc. Natl. Acad. Sci. U. S. A. 1999, 96, 8681-8686.

63. Hughes, L.A.; Melotte, V.; de Schrijver, J.; de Maat, M.; Smit, V.T.; Bovee, J.V.; French, P.J.; van den Brandt, P.A.; Schouten, L.J.; de Meyer, T. et al. The CpG Island Methylator Phenotype: What's in a Name? Cancer Res. 2013, 73, 5858-5868.

64. Greer, E.L.; Shi, Y. Histone Methylation: A Dynamic Mark in Health, Disease and Inheritance. Nature Reviews Genetics 2012, 13, 343-357.

65. Rodríguez-Rodero, S.; Delgado-Álvarez, E.; Fernández, A.F.; Fernández-Morera, J.L.; MenéndezTorre, E.; Fraga, M.F. Epigenetic Alterations in Endocrine-Related Cancer. Endocr. Relat. Cancer 2014, 21, R319-R330.

66. Drelon, C.; Berthon, A.; Mathieu, M.; Ragazzon, B.; Kuick, R.; Tabbal, H.; Septier, A.; Rodriguez, S.; Batisse-Lignier, M.; Sahut-Barnola, I. EZH2 is Overexpressed in Adrenocortical Carcinoma and is Associated with Disease Progression. Hum. Mol. Genet. 2016, 25, 2789-2800. 
67. Mariniello, B.; Rosato, A.; Zuccolotto, G.; Rubin, B.; Cicala, M.V.; Finco, I.; lacobone, M.; Frigo, A.C.; Fassina, A.; Pezzani, R. Combination of Sorafenib and Everolimus Impacts Therapeutically on Adrenocortical Tumor Models. Endocrine Related Cancer 2012, 19, 527.

68. Barlaskar, F.M.; Spalding, A.C.; Heaton, J.H.; Kuick, R.; Kim, A.C.; Thomas, D.G.; Giordano, T.J.; BenJosef, E.; Hammer, G.D. Preclinical Targeting of the Type I Insulin-Like Growth Factor Receptor in Adrenocortical Carcinoma. J. Clin. Endocrinol. Metab. 2009, 94, 204-212.

69. Jones, R.L.; Kim, E.S.; Nava-Parada, P.; Alam, S.; Johnson, F.M.; Stephens, A.W.; Simantov, R.; Poondru, S.; Gedrich, R.; Lippman, S.M. et al. Phase I Study of Intermittent Oral Dosing of the Insulin-Like Growth Factor-1 and Insulin Receptors Inhibitor OSI-906 in Patients with Advanced Solid Tumors. Clin. Cancer Res. 2015, 21, 693-700.

70. Fassnacht, M.; Berruti, A.; Baudin, E.; Demeure, M.J.; Gilbert, J.; Haak, H.; Kroiss, M.; Quinn, D.I.; Hesseltine, E.; Ronchi, C.L. et al. Linsitinib (OSI-906) Versus Placebo for Patients with Locally Advanced Or Metastatic Adrenocortical Carcinoma: A Double-Blind, Randomised, Phase 3 Study. Lancet Oncol. 2015, 16, 426-435.

71. Fraenkel, M.; Gueorguiev, M.; Barak, D.; Salmon, A.; Grossman, A.B.; Gross, D.J. Everolimus Therapy for Progressive Adrenocortical Cancer. Endocrine 2013, 44, 187-192.

72. Quinkler, M.; Hahner, S.; Wortmann, S.; Johanssen, S.; Adam, P.; Ritter, C.; Strasburger, C.; Allolio, B.; Fassnacht, M. Treatment of Advanced Adrenocortical Carcinoma with Erlotinib Plus Gemcitabine. J. Clin. Endocrinol. Metab. 2008, 93, 2057-2062.

73. Kroiss, M.; Quinkler, M.; Johanssen, S.; van Erp, N.P.; Lankheet, N.; Pollinger, A.; Laubner, K.; Strasburger, C.J.; Hahner, S.; Muller, H.H. et al. Sunitinib in Refractory Adrenocortical Carcinoma: A Phase II, Single-Arm, Open-Label Trial. J. Clin. Endocrinol. Metab. 2012, 97, 3495-3503.

74. Suh, I.; Weng, J.; Fernandez-Ranvier, G.; Shen, W.T.; Duh, Q.; Clark, O.H.; Kebebew, E. Antineoplastic Effects of Decitabine, an Inhibitor of DNA Promoter Methylation, in Adrenocortical Carcinoma Cells. Archives of Surgery 2010, 145, 226-232.

75. Mohan, D.R.; Lerario, A.M.; Hammer, G.D. Therapeutic Targets for Adrenocortical Carcinoma in the Genomics Era. Journal of the Endocrine Society 2018, 2, 1259-1274.

76. Creemers, S.; Van Koetsveld, P.; Van Den Dungen, E.; Korpershoek, E.; van Kemenade, F.; Franssen, G.; de Herder, W.; Feelders, R.; Hofland, L. Inhibition of Human Adrenocortical Cancer Cell Growth by Temozolomide in Vitro and the Role of the MGMT Gene. The Journal of Clinical Endocrinology \& Metabolism 2016, 101, 4574-4584.

77. Hegi, M.E.; Diserens, A.; Gorlia, T.; Hamou, M.; De Tribolet, N.; Weller, M.; Kros, J.M.; Hainfellner, J.A.; Mason, W.; Mariani, L. MGMT Gene Silencing and Benefit from Temozolomide in Glioblastoma. N. Engl. J. Med. 2005, 352, 997-1003.

78. Quan, C.; Xiao, J.; Duan, Q.; Yuan, P.; Xue, P.; Lu, H.; Yan, M.; Guo, D.; Xu, S.; Zhang, X. et al. T-Lymphokine-Activated Killer Cell-Originated Protein Kinase (TOPK) as a Prognostic Factor and a Potential Therapeutic Target in Glioma. Oncotarget 2017, 9, 7782-7795.

79. Cosentini, D.; Badalamenti, G.; Grisanti, S.; Basile, V.; Rapa, I.; Cerri, S.; Spallanzani, A.; Perotti, P.; Musso, E.; Laganà, M. Activity and Safety of Temozolomide in Advanced Adrenocortical Carcinoma Patients. European Journal of Endocrinology 2019, 1.

80. Charlet, J.; Schnekenburger, M.; Brown, K.W.; Diederich, M. DNA Demethylation Increases Sensitivity of Neuroblastoma Cells to Chemotherapeutic Drugs. Biochem. Pharmacol. 2012, 83, 858-865.

81. Strauss, J.; Figg, W.D. Using Epigenetic Therapy to Overcome Chemotherapy Resistance. Anticancer Res. 2016, 36, 1-4.

82. Grisanti, S.; Cosentini, D.; Laganà, M.; Abate, A.; Rossini, E.; Sigala, S.; Berruti, A. Are we Failing in 
Treatment of AdrenoCortical Carcinoma? Lights and Shadows of Molecular Signatures. Current Opinion in Endocrine and Metabolic Research 2019.

83. Crona, J.; Beuschlein, F. Adrenocortical Carcinoma - Towards Genomics Guided Clinical Care. Nature Reviews Endocrinology 2019, 15, 548-560.

84. Koch, A.; Joosten, S.C.; Feng, Z.; de Ruijter, T.C.; Draht, M.X.; Melotte, V.; Smits, K.M.; Veeck, J.; Herman, J.G.; Van Neste, L. Analysis of DNA Methylation in Cancer: Location Revisited. Nature Reviews Clinical Oncology 2018, 15, 459-466.

85. De Ruijter, T.C.; De Hoon, J.P.; Slaats, J.; De Vries, B.; Janssen, M.J.; Van Wezel, T.; Aarts, M.J.; Van Engeland, M.; Tjan-Heijnen, V.C.; Van Neste, L. Formalin-Fixed, Paraffin-Embedded (FFPE) Tissue Epigenomics using Infinium HumanMethylation450 BeadChip Assays. Laboratory investigation 2015, 95, 833-842.

86. Thirlwell, C.; Eymard, M.; Feber, A.; Teschendorff, A.; Pearce, K.; Lechner, M.; Widschwendter, M.; Beck, S. Genome-Wide DNA Methylation Analysis of Archival Formalin-Fixed Paraffin-Embedded Tissue using the Illumina Infinium HumanMethylation27 BeadChip. Methods 2010, 52, 248-254.

87. Mohan, D.R.; Lerario, A.M.; Else, T.; Mukherjee, B.; Almeida, M.Q.; Vinco, M.; Rege, J.; Mariani, B.M.; Zerbini, M.C.N.; Mendonca, B.B. Targeted Assessment of GOS2 Methylation Identifies a Rapidly Recurrent, Routinely Fatal Molecular Subtype of Adrenocortical Carcinoma. Clinical Cancer Research 2019, 25, 3276-3288.

88. Fragoso, M.C.B.; Almeida, M.Q.; Mazzuco, T.L.; Mariani, B.M.; Brito, L.P.; Gonçalves, T.C.; Alencar, G.A.; Lima, L.d.O.; Faria, A.M.; Bourdeau, I. Combined Expression of BUB1B, DLGAP5, and PINK1 as Predictors of Poor Outcome in Adrenocortical Tumors: Validation in a Brazilian Cohort of Adult and Pediatric Patients. European journal of endocrinology 2012, 166, 61.

89. Tissier, F. Pathological Pattern of Adrenal Cortical Carcinoma. 2010. 
Part III

Elaboration 


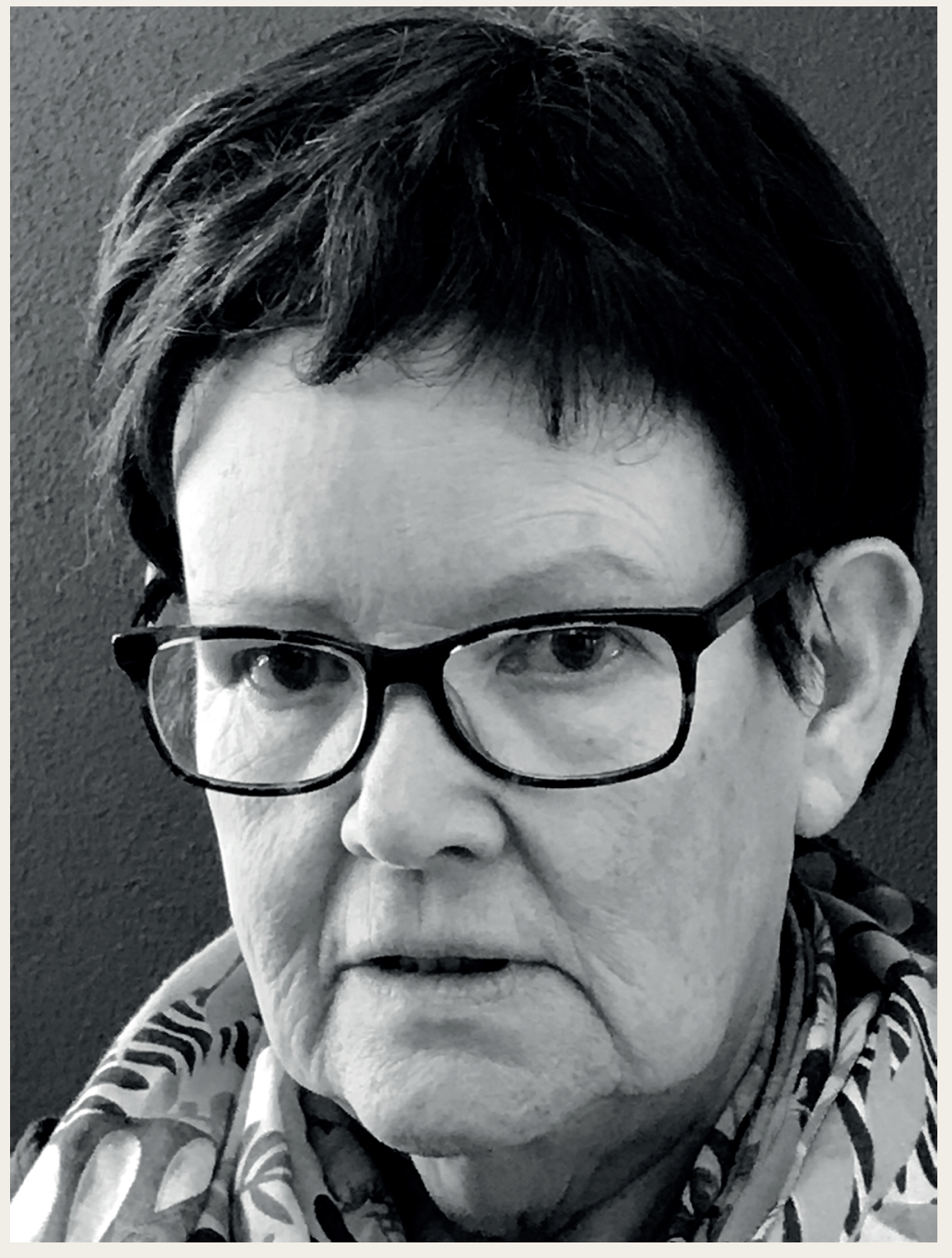




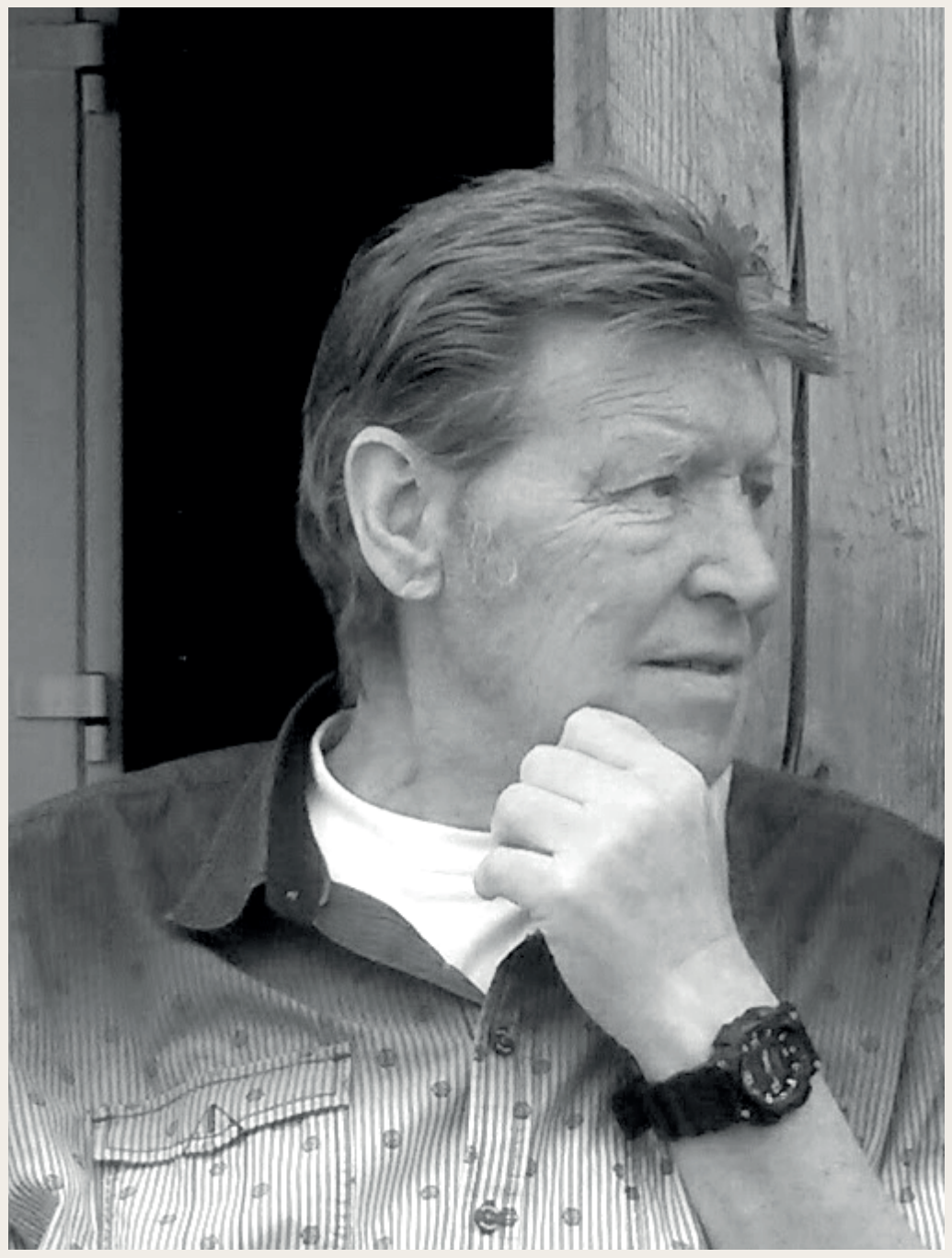


Chapter 07

General discussion and future perspectives 


\section{General Discussion}

This thesis focuses on prediction. The prediction of the pathologic behavior and clinical outcome of ACC, the treatment strategy of mitotane as cornerstone in the management of ACC and the future of treating ACC. In this chapter the main findings of this thesis are discussed in the light of current literature. Furthermore, strategies and topics for future research are explored (Table Future directions in ACC).

\section{Predicting Clinical Course in Adrenocortical Carcinoma}

Adrenocortical carcinoma prognosis is grim and varies widely. It ranges from tumors curable by complete surgery to those that are resectable but with high recurrence rate, or unresectable, fast growing and with an aggressive potential for metastatic spread. Reliable prognostication in ACC is critical to patients and treating physicians for making decisions regarding (adjuvant) treatment, type, and frequency of followup, as well as for providing patients with helpful information about short-term and long-term outcomes and to make a shared decision concerning treatment modalities.

Current prognostic factors include tumor stage, best assessed by the European Network for the Study of Adrenal Tumors (ENSAT) and tumor proliferation index measured by Ki67 immunostaining. Other commonly reported prognostic factors include cortisol secretion and age.

In chapter 2 it is shown that synchronous metastases of ACC are associated with a worse prognosis compared to metachronous metastases of ACC and patients with late synchronous metastases have a better prognosis than those with metastases at initial diagnosis. Furthermore, number of affected organs and number of metastases are associated with poor prognosis. Interestingly, it is shown that adrenalectomy had a strong positive influence on survival, even in the presence of metastases. This result should be interpreted cautiously, because it could be hypothesized that patients with stage IV amendable to surgery are in a condition that allows an operative intervention and therefore have better overall survival compared to other patients with advanced disease not amendable for surgery. However, positive results on surgical management in advanced disease has been observed in other studies (1-4). Current guideline suggest against the routine use of adrenal surgery in case of widespread metastatic disease. Still, the guideline states that in selected cases (e.g. patients with severe hormone excess) debulking surgery might be an option, taking anti-hormonal drugs into account, reasoning that surgery might be appropriate if $>80 \%$ of the tumor burden can be removed safely (5).

In chapter $\mathbf{3}$ a prediction model is presented capable of predicting ACC-specific mortality which included age, modified European Network for the Study of Adrenal Tumors (mENSAT) stage, and radical resection. 
Over the years many attempts have been made to identify clinical parameters and pathological index/scores, for the prediction of clinical outcome in ACC, but accurate prognostication remains a challenge. It seems that for stage I-II risk of recurrence after resection is the most important question whereas for stage III and IV survival is more relevant.

ENSAT stage unmistakably holds up in many studies as predictor of survival (see appendix 3 supplementary data to Fassnacht et al. 2018@, European Society of Endocrinology Clinical Practice Guidelines on the Management of Adrenocortical Carcinoma in Adults) (5-7). In chapter 2 we found that spread to lymph nodes affects survival to the same extent as hematogenous metastasis. Other studies have found similar negative effects of nodal metastasis on survival $(8,9)$. Libé et al. refined the ENSAT stage and suggested mENSAT. In this new classification, the N1 status shifted from ENSAT stage III to the mENSAT stage IV category, with Stage III defined as T3-4NOMO and stage IVa, IVb, IVc according to the number of tumor-involved organs (including the primary tumor and the ' $\mathrm{N}$ ' as 'organ'): two, three or more than three, respectively(10). Increasing evidence suggested beneficial prognostic impact of a prophylactic loco regional lymphadenectomy during primary tumor resection, where it has been found to improve tumor staging and survival outcome in patients with localized ACC $(11,12)$. It was even added to the European guidelines on the management of ACC (2018): routine loco-regional lymphadenectomy should be performed with adrenalectomy for highly suspected or proven ACC, including (as a minimum) the periadrenal and renal hilum nodes. Furthermore, it is advised that all suspicious or enlarged lymph nodes identified on preoperative imaging or intraoperatively should be resected $(5,9,11,13)$. The importance of surgery in the management of ACC is also illustrated by its impact on oligometastatic disease. In chapter 2 we noticed a positive effect of a metastasectomy in patients with limited spread of disease to the lungs, on survival. Other studies have found similar results $(2,14,15)$, endorsing prospective studies on the role of oligometastasectomy in ACC in well-defined study and control groups.

In chapter 3 it is shown that the mENSAT performed slightly better than ENSAT in a clinical prediction model. Other modifications of the ENSAT have been proposed. Asare et al. suggested adding age to the ENSAT staging for better prediction of overall survival (OS) in stage I-II ACC (16). Miller et al. suggested the Ann Arbor modification of the ENSAT staging system incorporating tumor grade (17). Still none of these staging systems has been implemented in the guideline. Insufficient prospective validation maybe the reason. Also, some studies did not find age/tumor grade to be of impact on prognosis. Studies that included multivariate models did not find age as a predictor of recurrence $(6,18,19)$ or cancer-specific mortality $(20)$. Age could be associated with an increase in all-cause mortality due to an increase in treatment-related mortality or in non-specific mortality. It just could be a confounding factor of frailty and 
comorbidities and subsequent treatment decisions. But it should be acknowledged that recurrence is a different outcome measure than overall survival. Presumably, to predict recurrence and overall survival in ACC accurately, separate prognostic models are needed. Implementing multiple models could be an inconvenience and confusing when used in clinical practice.

The problem with tumor grade, defined as Ki67, as prognostic factor is high interand intra-observer variability (21) and limited reproducibility. The heterogeneity in the preanalytical process (fixation) and immunostaining is not completely standardized and might affect Ki67 measurement $(22,23)$. It has been suggested that digital microscopy-enabled methods could provide critical aid in reducing variation, increasing reproducibility. In 2014 an open source automated detection quantitative ranking of hotspots was published to support histopathologists in selecting the 'hottest' hotspot areas, but this has not been implemented in daily practice $(21,24)$. Even when standardized, interpretation of Ki67 staining and scoring may be subjected to predicaments: Does the sample analyzed represent the whole tumor? How many cells or high-power fields have to be included for evaluation? Do we need to score Ki67 into hot spot areas, or average score across the section? And do we have to score multiple samples from one tumor? And if so, do we take the average of all the samples or the highest score measured? These difficulties may explain why in chapter 3 we are missing so many Ki67 data. This calls for a more objective and reproducible prognostic marker, in which there is a potential role for genomics (discussed later). Also, as we plead for in chapter 5, a standardized pathology record would guarantee a complete report, containing itemized Weiss-score and standardized assessment of Ki67-index. On the other hand, it could be argued that the best prediction model is one that is easy to use in clinical practice and therefore consist of 'simple' prognosticators as we showed in chapter 3.

The impact of cortisol secretion of the tumor remains uncertain in ACC. It appears that it mostly negatively impacts patients undergoing surgery $(19,25)$, whereas it showed no prognostic value in other series involving localized (6) or metastatic ACC patients (26). It could be suggested that hypercortisolism, as implied for age, is associated with a general increase in mortality. Hypercortisolism is associated with an increased risk of thromboembolism, and it suppresses the immune system increasing the risk of infection and post-operative complications.

In addition, it should be noted that there is statistical difference in showing an association of a variable in relation to outcome using a regression analysis or creating a predictive formula or nomogram that can calculate a predefined outcome for an individual patient. Most important, a general concern for studies on prognosis is the number of variables included in models, relative to the number of events, because when outbalanced this may have the potential to lead to false-positive results. 


\section{Improvement of current strategies and developing new treatments}

Although prognostication allows for a more personalized treatment strategy, it has not generated new therapeutic options. Fortunately, the survival rate of ACC has slightly improved over the last years. Does this mean we are treating patients better? The reasons are not totally clear, studies have shown that a higher percentage of patients are diagnosed in earlier stages (27) which may be explained by improved imaging techniques.

Also, the improvement of surgical techniques with a complete tumor removal even for advanced disease stages, or the widespread use of adjuvant therapy with mitotane may explain, at least in part, this positive evolution. Possibly, the repeat use of local therapy in selected patients might have led to prolonged stabilization and control of the disease $(28,29)$. Moreover, it could be hypothesized that the appeal of treating ACC patients in dedicated centers might have positively contributed to the referral, and the improvement of, multidisciplinary expert team meetings, consequently leading to better individualized (multidisciplinary) treatment strategies and hence better outcome.

In chapter 5 future ACC diagnostics, prognostics and treatment concepts are discussed. It is disappointing that since this publication, despite effort, no new therapies have been established for ACC. Understanding why we are failing in treatment in ACC is key. Drug resistance is a very important part of this challenge. ACC has a medium-to-low sensitivity to alkylating drugs (cisplatin, streptozotocin), drugs interfering with DNA-topoisomerase (etoposide, doxorubicin, irinotecan) or drugs interfering with DNA bases (gemcitabine, capecitabine) (30). Also attempted targeted therapy, aimed at three most altered pathways in ACC (IGF2, Wnt/beta catenin and TP53) failed to show significant improvement of survival. Overexpression of a molecular pathway as a result of one single genetic alteration has been proven insufficient for making a good therapeutic target for ACC (e.g. linsitinib trial aimed at the IGF1R (31), everolimus aimed at mTOR (32)) or gefitinib and erlotinib aimed at the EGFR pathway $(33,34)$. Tumor heterogeneity between patients, but also heterogeneity among different metastatic sites within the same patient, pose a problem with this single pathway approach. Unfortunately, the identification of targets linked to the multiple driver molecular alterations are lacking.

In the meantime, mitotane remains the main therapeutic backbone of ACC and actually still is the only Food and Drug Administration (FDA)/European Medicines Agency (EMA)-approved drug. It is used treating ACC in either adjuvant or metastatic setting. Therefore, improving mitotane treatment is a logical consequence. Our current clinical knowledge on mitotane is mostly based on retrospective studies. The exact mechanism of action of mitotane is not yet fully understood and remains an object of investigation as well as how it influences other therapeutic agents when given concomitantly. 
Mitotane is a complicated drug. Its effectiveness is correlated with a therapeutic level of 14 mg/l (35-37). Unfortunately most patients require a long treatment period to reach those levels because of unfavorable pharmacokinetics of mitotane: low oral bioavailability of 30-40\% (38), high volume of distribution due to its high lipophilicity, and its elimination half-life varying between 18-159 days (39-41). Almost all patients experience side effects. In the study by Daffara et al. 76\% of patients even experienced multiple side effects.

Earlier it was shown by our group that weight, body surface area (BSA) and lean body weight (LBA) were significantly correlated with volume of distribution in the central compartment (V1) in a pharmacokinetic model for mitotane (42). However, it was acknowledged that a residual variance in the population pharmacokinetic (PK) parameters was not explained by clinical parameters and it was hypothesized that genetic factors are involved in mitotane pharmacokinetics.

Earlier, two studies suggested possible roles for CYP2B6 and CYP2C9 (43). One study demonstrated that the genotype of CYP2B6*6 (rs3745274) was significantly correlated with mitotane plasma concentrations at 3 and 6 months after initiation of treatment (44). However, after nine months, treatment difference was no more significant. Also, they only investigated CYP2B6 and ABCB1. The other study showed that one patient with high mitotane concentration was a CYP2C9 intermediate metabolizer (43).

In chapter 4 we showed that lean body weight (LBW), genotypes of CYP2C19*2 (rs4244285), SLCO1B3 699A>G (rs7311358), and SLCO1B1 571T>C (rs4149057) were identified to affect mitotane clearance (CL/F) significantly, which decreased the coefficient of variation (CV\%) of random inter-individual variability of CL/F from $67.0 \%$ to $43.0 \%$. Fat amount was identified to affect the central distribution volume significantly. A major limitation of this study is that the effect of co-medication was not included in the analysis because of missing data. Mitotane is a known strong inducer of CYP3A4 (45). Van Erp et al. found an increased metabolism of midazolam, when given concomitantly with mitotane, indicative of CYP3A4 upregulation (45). In addition, it has been demonstrated that mitotane induced liver microsomal enzymes and, more specifically, CYP3A4 through binding to the pregnane X receptor (PXR) in liver cells $(46,47)$. Considering that CYP3A4 is one of the most important drug-metabolizing microsomal monooxygenases, which is considered to be implicated in the metabolism of about $50 \%$ of the drugs on the market, many drugs potentially are affected by mitotane-related CYP3A4 induction(48). It could also be hypothesized that this (partially) explains the therapeutic failure of tested targeted pharmaceuticals in ACC, e.g. everolimus (32), an mTOR inhibitor, imatinib (49), a tyrosine kinase inhibitor and gefitinib (34), an inhibitor of epidermal growth factor (EGR). All these drugs are substrates of CYP3A4, and hence, insufficient drug exposures may have been reached in these clinical trials, since patients were treated with mitotane in the recent past, and therefore with measurable levels in blood because of the long elimination half-life, or even concomitantly. 
In the case of PK modelling it is more interesting to know whether co-medication influences mitotane. Statins are known to promote the effect of mitotane. In a retrospective study by Hescot et al. 26 patients with ACC the combination of mitotane and statins (Rosuvastatin not metabolized by CYP3A4 but, presumably, CYP2C9 and CYP2C19) was shown to be significantly associated with a better tumor control according to Response Evaluation Criteria in Solid Tumors criteria (RECIST) (50). Boulate et al recently showed in $\mathrm{NCl}-\mathrm{H} 295 \mathrm{R}$ human ACC cells that rosuvastatin potentiated the effects of mitotane by reducing cell viability, inducing apoptosis, increasing intracellular free cholesterol levels, and by decreasing the expression of 3-hydroxy-3-methylglutaryl-CoA reductase (HMGCR) and ATP binding cassette subfamily a member 1 (ABCA1), genes involved in cholesterol metabolism, and inhibiting steroidogenesis(51). As mentioned earlier, CYP2C9 has been correlated with mitotane levels (43) and we show in chapter 4 a role for CYP2C19 in the clearance of mitotane. Both enzymes are involved in the metabolism of rosuvastatin, perhaps contributing to their synergy.

Noteworthy, mitotane induces dyslipidemia with increased LDL, HDL and triglycerides concentrations $(52,53)$. Remarkably, this dyslipidemia reduces mitotane efficacy in vitro as suggested by the higher anti-proliferative and pro-apoptotic effects of mitotane when NCl-H295R cells are cultured in a lipoprotein-free medium (50). Also, hyperlipidemia leads to an overestimation of plasma mitotane levels in patients (54). Further research should focus on the understanding of how mitotane is associated with lipid changing on the one hand and the mechanism through which statins may potentiate the effects of mitotane on the other hand.

In contrast to what we already know about drug synergism and effect of mitotane on other medication, little is known about medication that reduce mitotane efficacy. It has been suggested that spironolactone, commonly used in the management of the hypokalemia of Cushing's syndrome, blocks the action of mitotane and should be avoided if possible (55).

Mitotane is advised to be taken with food, preferably a fatty meal, where this could enhance absorption (39). When taking this into account it could be hypothesized that also the acidity of gastric fluid might affect mitotane absorption. However, the role of proton pump inhibitors (PPI) and their influence on mitotane absorption has not been investigated. While for many other medications, e.g. ketoconazole, it is known that absorption is rendered when given in combination with a PPI (56).

For some drugs synergism with mitotane have been proposed. Kroiss et al. showed a synergism of bortezomib and carfilzomib, proteasome inhibitors who indirectly activate endoplasmic reticulum (ER) stress, with mitotane in the $\mathrm{NCl}-\mathrm{H} 295$ cell culture model for ACC (57). Such enhancement of the effect of mitotane could be clinically very relevant, where only half of patients treated with mitotane reach therapeutic levels (58). 
It can be concluded that therapeutic drug monitoring (TDM) is very important not only for mitotane but also for certain concomitant given drugs. In our experience, the major problem with TDM is the delay in test results for mitotane. This is caused by two factors: firstly, the concentration of mitotane in blood can only be determined in one medical center in the Netherlands, and it generally takes one week from submitting a blood sample to receiving the result. The alternative, Lysosafe ${ }^{\circledR}$ (Lysosafe is a is a free of charge mitotane plasma level testing service in France offered by HRA Pharma - the pharmaceutical producing mitotane $\left\{\right.$ Lysodren $\left.{ }^{\circledR}\right\}$ - to all hospitals in Europe using Lysdoren ${ }^{\circledR}$ in the treatment of ACC), is not quicker. Secondly, in our treatment protocol the first blood sample is taken two to four weeks after starting mitotane treatment in the building up phase. So, when the result is in, we might find that the patient may have received too little or too much mitotane for at least three weeks. A good example is the case report of Di Paolo et al. they showed that thanks to TDM their patient did not have Central Nervous System (CNS) or gastrointestinal toxicities, but this took a long time of dose adjusting (six months) (59).

The Population PK (PopPK) model proposed in chapter 4 could fill this hiatus, where it could allow for the predicting of mitotane levels, considering previous individual plasma level measurements as well.

Predicting mitotane levels in advance allows for an increase in dosage when the predicted level is not at a therapeutic level of $14 \mathrm{mg} / \mathrm{l}$ within expected time frame, or to lower the dosage when it is expected that toxic levels above $20 \mathrm{mg} / \mathrm{l}$ will be reached. Off course in the latter situation, side effects experienced by the patient should be weighed, because when levels of 20 or above are well tolerated by the patient, continuing the dosage can be considered. Further, it could allow for a personalized dosage regimen to build up to a therapeutic level and inform about the dosage needed to maintain a therapeutic range. Currently there is no consensus regarding the optimal dosage strategy for mitotane which depends on a physician's expertise and is therefore prone to error $(58,60)$. A median of three to five months is reported in studies $(36,61)$ to reach therapeutic levels. This can be an unacceptable long time for patients with advanced disease or at high risk of recurrence. Besides supportive medication to temper experienced side effects it could be hypothesized that a predictive PK model could motivate the patient to continue therapy knowing they will reach a therapeutic level with the advised dosage regimen. In the study by Mauclère-Denost et al. was transiently discontinued $50 \%$ of patients, due to toxicity (60) and also Kerkhofs et al. report about the discontinuation of mitotane treatment (58). Discontinuation should be minimized by regular counseling about the management of adverse effects, careful adjustment of hormone replacement therapies, and tailoring of mitotane dosage. The latter could be based on the PK model in which the measured levels can be entered, and, of course considering the experienced side effects. For this prospective validation of our presented PK model is warranted. 


\section{Molecular profiling/biomarkers}

In the last decades, multiple molecular alterations have been found to promote ACC carcinogenesis. Understanding ACC tumorigenesis is of importance where it might yield the identification of new markers that improve diagnostic accuracy, predict treatment response or even provide novel therapeutic targets.

In chapter 6 an overview of new insights in the evolving field of epigenetic studies on ACC is provided. Epigenetics involves genetic modification without altering the DNA sequence. Most epigenetic changes concern DNA methylation, attaching methyl groups to segments of DNA, and histone modification. Hypermethylation is a common phenomenon seen in cancer. It has been found that there is significantly more hypermethylation at CpG sites in ACC. Different studies were able to find such CpG clusters to correlate with ACC survival, so called CIMP-high (poor prognosis) and CIMP-low clusters (62-64). Also, studies have identified that expression levels of, on mRNA levels of mitotic regulator, BUB1 Mitotic Checkpoint Serine/Threonine Kinase B (BUB1B) and, mitochondrial kinase PTEN Induced Putative Kinase 1 (PINK1), help in identifying different subgroups of ACC with different survival rates $(65,66)$.

The term CIMP, often referring to the CpG island promoter methylation, is controversial since there is actually no universal standard or consensus with respect to defining CIMP. It is used to describe the increased prevalence of CpG island promoter methylation. CpG islands are genomic regions larger than 500 base pairs (bp). More than 55\% of these nucleotides are composed of CpG dinucleotides (67).

As Koch et al. already advocated at the beginning of 2018, international effort needs to be made to make molecular profiling of clinical relevance (68). They reported that in January 2018 there were more than 14,000 scientific publications describing DNA methylation- based biomarkers and their clinical associations in cancer, but only 14 of these biomarkers had been translated into a commercially available clinical test. They recommend that the location of a potential DNA methylation biomarker and the number of CpGs to cover should be critically evaluated. Most genetic studies on ACC clearly describe the type of (Infinium) assay (see chapter $\mathbf{6}$ ). There is heterogeneity in the microarrays used and therefore in the results. Also, the microarray restricts data to the particular genomic locations of the probes used in the array, which might not necessarily capture the most relevant methylation sites. Microarrays that cover over 850,000 CpG methylation sites (850K) have shown to also find CpG clusters not only in islands but also $\mathrm{CpG}$ shores (sequences 2 kilobytes (kb) upstream and downstream from CpG island), in CpG shelves (sequences $2 \mathrm{~kb}$ upstream and downstream from shore regions) and outside these regions ('open sea').

What is missing in most studies on ACC is an accurate and thoroughly report on the exact genomic location of the biomarker, which is depending on the microarray and 
cut-off values used.

However, it seems that we are on the verge of the development of a biomarker for prognosis in ACC. Despite the methodologic differences the CIMP phenotype has been shown in three different studies.

The development of a biomarker that can clearly distinguishes ACA and ACC (potentially BUB1-PINK1 (69)), predicts recurrence or treatment response in ACC appears more challenging. Sterol-O-acyl- transferase 1 (SOAT1) has been suggested a potential biomarker for treatment response of mitotane (70).

Most important, multi-omics studies are establishing insight into the heterogeneous and complex molecular biology of ACC and identifying drug targets (see table below). Unfortunately, for many of these targets no treatment is available yet (see table 3 Crona \& Beuschlein 2019C, Adrenocortical carcinoma - towards genomics guided clinical care, Nature Endocrinology (71)). Increasingly, it is stated that ACC research should be focused on biomarker driven clinical trials. This means international effort should be made to implement biomarkers in the guideline and, as a result, daily practice.

\begin{tabular}{|c|c|c|}
\hline $\begin{array}{l}\text { Drug target (mutation } \\
\text { frequency in ACC) }\end{array}$ & Therapy & Studies \\
\hline $\begin{array}{l}\operatorname{NF1}(17 \%)(71,72) \\
\operatorname{ATM}(3-10 \%)(71,72)\end{array}$ & $\begin{array}{l}\text { Everolimus, } \\
\text { Temsirolimus }\end{array}$ & $\begin{array}{l}\text { Fraenkel et al. } 2013 \text { - Everolimus: } \\
\text { clinically no meaningful response } \\
\text { was observed in four patients with } \\
\text { advanced } \text { AC }^{\star}(32) \text {. }\end{array}$ \\
\hline $\begin{array}{l}\operatorname{CDKN} 2 A / B(11-15 \%)(63,64) \\
\operatorname{CDK} 4(2-7 \%)(63,72) \\
\operatorname{ATM}(10 \%)(71,72)\end{array}$ & $\begin{array}{l}\text { CDK 4/6 inhibitors: } \\
\text { Abemaciclib } \\
\text { Palbociclib } \\
\text { Ribociclib }\end{array}$ & $\begin{array}{l}\text { Fiorentini et al. } 2018 \text { - Palbociclib: } \\
\text { significantly affected cell viability in } \\
\text { NCI-H295R cell line for ACC (73). }\end{array}$ \\
\hline PTCH1 (3\%)(71,72) & Vismodegib & $\begin{array}{l}\text { LoRusso et al. } 2011 \text { - Phase I } \\
\text { trial Vismodegib: included } 2 \text { ACC } \\
\text { patients. Progressive disease/ } \\
\text { missing, no details specified (74). }\end{array}$ \\
\hline $\begin{array}{l}\text { * Patients used mitotane, } \\
\text { potentially diminishing } \\
\text { therapeutic effect of Everolimus. }\end{array}$ & & \\
\hline
\end{tabular}

\section{Future perspectives}

Adrenocortical carcinoma is an orphan disease. Collaborative efforts, like the European Network for the Study of Adrenal Tumors (ENSAT), and the more recent established A5: American-Australian-Asian Adrenal Alliance, are making important contributions in the research field of ACC. Such strong international collaborations and large consortia are 
necessary to improve diagnostic and therapeutic strategies for patients with ACC.

The Dutch Adrenal Network (DAN) should make an effort to initiate a national biobank. The prognostic model presented in this thesis had a disadvantage because of lacking Ki67 data as a result of pathology records missing or Ki67 not evaluated and therefore not mentioned in the pathology report. An attempt to retrieve these tumor samples for revision proved to be very difficult with material being unavailable. A centralized biobank could overcome this problem, making the storage and accessibility of patient samples easier for research purposes. Additionally, a standardized pathology report for ACC could ensure a thorough report including Weiss and Ki67.A potential improvement would be a national collaboration between the Netherlands Cancer Registry, which is managed by the Netherlands Comprehensive Cancer Organization (IKNL), and the DAN.

Prognostication of ACC has improved with the introduction of the ENSAT-staging and the proliferation marker, Ki67. Recently there have been relevant publications that ought to further improve prognostication. This is necessary, because predicting recurrence still is problematic, as is the heterogeneous prognosis within the different ENSAT stages. It appears that both selected clinical factors (e.g. Resection, Age, Symptoms) and molecular biomarkers (COMBI score) have the potential to improve prognostication. Prospective trials, with large patient cohorts are needed to further investigate the discriminative value of these prognostic factors.

New therapeutic strategies in ACC should be stratified according to patients' molecular subtype by using thorough validated biomarkers that permit prospective assessment. It must be stressed that individual therapeutic approaches are unlikely to be efficacious in all ACC subtypes. An interesting approach has been suggested by Crona \& Beuschlein. They argue that a, so called, umbrella clinical trial design. This design, in which a distinct disease is selected for study and patients are stratified on the basis of molecular data towards different treatment baskets, would allow researchers to study the feasibility and efficacy of the entire concept of geneticsguided therapy rather than evaluating different agents separately, each with very low numbers of patients (71).

Furthermore, it could be thoroughly considered whether new agents should be tested concomitant with mitotane. Because of the long elimination half-life of mitotane, after termination, an interaction could be expected for a long period.

For many years it has been accepted that one type of medication is given to various types of patients. With the upcoming field of pharmacogenetics, we are entering a new era of personalized medicine.

For mitotane it could allow for a personalized dosage strategy in order to attain a therapeutic level within three months preferably. We showed that CYP2C19*2, 
SLCO1B3 699A>G and SLCO1B1 571T>C influence pharmacokinetics of mitotane. Further validation is needed to confirm these findings in an independent cohort.

Considering that mitotane remains important in the therapy of ACC, effort should be made to better understand its mechanism of action. Markers that predict therapeutic efficacy would help deciding on adjuvant therapy. Investment should be made to improve mitotane treatment form. Its current form as a tablet of 500 milligram is not very patient friendly, considering taking up to 24 tablets a day is necessary, and uptake is reliant on a patients' diet. Intravenous treatment with mitotane in a Lipomul suspension is described in only one study in 1961 (38) and failed to be superior to oral treatment. Lipid-carriers or special tablet coatings could enhance the uptake and pharmacokinetics of mitotane.

Finally, and maybe most important, we need to gain insight into the health-related quality of life (HRQoL) impact of adrenocortical carcinoma (ACC). For patients with a rare disease, limited treatment options with high treatment burden and limited survival HRQOL research is of utmost importance. Shared decision making, as opposed to clinicians making decisions, requires that a healthcare professional should know how the course of the disease and the treatments of ACC impact a patients HRQoL. When discussing diagnosis and treatment options, respecting ones wishes, treatment strategy should be aimed at improving quality of life and enable well-being where possible. This again emphasizes that ACC care should be managed by a multidisciplinary expert team.

Those medical experts, together in teams, may have improved ACC survival over the last couple of years, and it's their collaboration that brings hope for the future. 


\begin{tabular}{|c|c|c|}
\hline Diagnostics & Prognostics & Therapy \\
\hline \multicolumn{3}{|c|}{ Implementation necessary } \\
\hline $\begin{array}{l}\text { - Implementation of a } \\
\text { standardized (pre)analytic } \\
\text { protocol for Ki67 in ACC. } \\
\sim \text { Line number } 7 \sim \\
\text { - Implementation of a }\end{array}$ & $\begin{array}{l}\text { - Implementation of a multi- } \\
\text { omic molecular panel with } \\
\text { the best selected biomarker } \\
\text { predictors. Line number } \\
\text { 280 }\end{array}$ & $\begin{array}{l}\text { - Implementation of a combine } \\
\text { pharmacokinetic and genetic } \\
\text { model for personalized } \\
\text { dosage of mitotane. Line } \\
\text { number 156, Chapter 4 }\end{array}$ \\
\hline \multicolumn{3}{|c|}{ Validation required } \\
\hline & $\begin{array}{l}\text { - Validate mENSAT in a } \\
\text { prospective cohort. Line } \\
\text { number 57 } \\
\text { - Validate Grade, Resection } \\
\text { status, Age, Symptoms (GRAS) } \\
\text { criteria in a prospective } \\
\text { cohort. Chapter 3 }\end{array}$ & $\begin{array}{l}\text { Mitotane } \\
\text { - Prospective } \\
\text { of the single nucleotide } \\
\text { polymorphisms of the } \\
\text { CYP enzymes identified in } \\
\text { chapter 4 Line number } 241 \text {, } \\
\text { chapter 4 }\end{array}$ \\
\hline & $\begin{array}{l}\text { - Comprehensive international } \\
\text { prospective validation } \\
\text { of multi-omic predictive } \\
\text { markers. C Chapter } 6 \sim\end{array}$ & $\begin{array}{l}\text { - Prospective validation of } \\
\text { the two compartment } \\
\text { pharmacokinetic model } \\
\text { of mitotane presented in } \\
\text { chapter } 4 \sim \text { Line number } \\
\text { 241 }\end{array}$ \\
\hline \multicolumn{3}{|c|}{ Need for future studies } \\
\hline $\begin{array}{cr}\text { - Prospective } & \text { comparison } \\
\text { of current } & \text { pathologic } \\
\text { classification } & \text { (Ki67 }\end{array}$ & $\begin{array}{l}\text { - Consider a predictive model } \\
\text { combining clinical, pathologic } \\
\text { predictors and biomarkers. }\end{array}$ & $\begin{array}{l}\text { Prospective study on the role } \\
\text { of adrenalectomy in stage IV } \\
\text { patients. Line number 19 }\end{array}$ \\
\hline $\begin{array}{l}\text { and Weiss score) of } \\
\text { adrenal tumors versus a } \\
\text { genomic assay versus the } \\
\text { combination of both in } \\
\text { the process of accurately } \\
\text { diagnosing adrenal } \\
\text { tumors. Line number 86 }\end{array}$ & 〜Chapter 6 & $\begin{array}{l}\text { Prospective study on the role } \\
\text { of a metastasectomy in well- } \\
\text { defined study and control } \\
\text { groups of ACC patients. Line } \\
\text { number 55 }\end{array}$ \\
\hline \multicolumn{3}{|c|}{ Necessity for future initiatives } \\
\hline $\begin{array}{l}\text { - Centralized biobank in the } \\
\text { Netherlands for adrenal } \\
\text { tumors. Line number } \\
\text { 314 }\end{array}$ & & \\
\hline
\end{tabular}




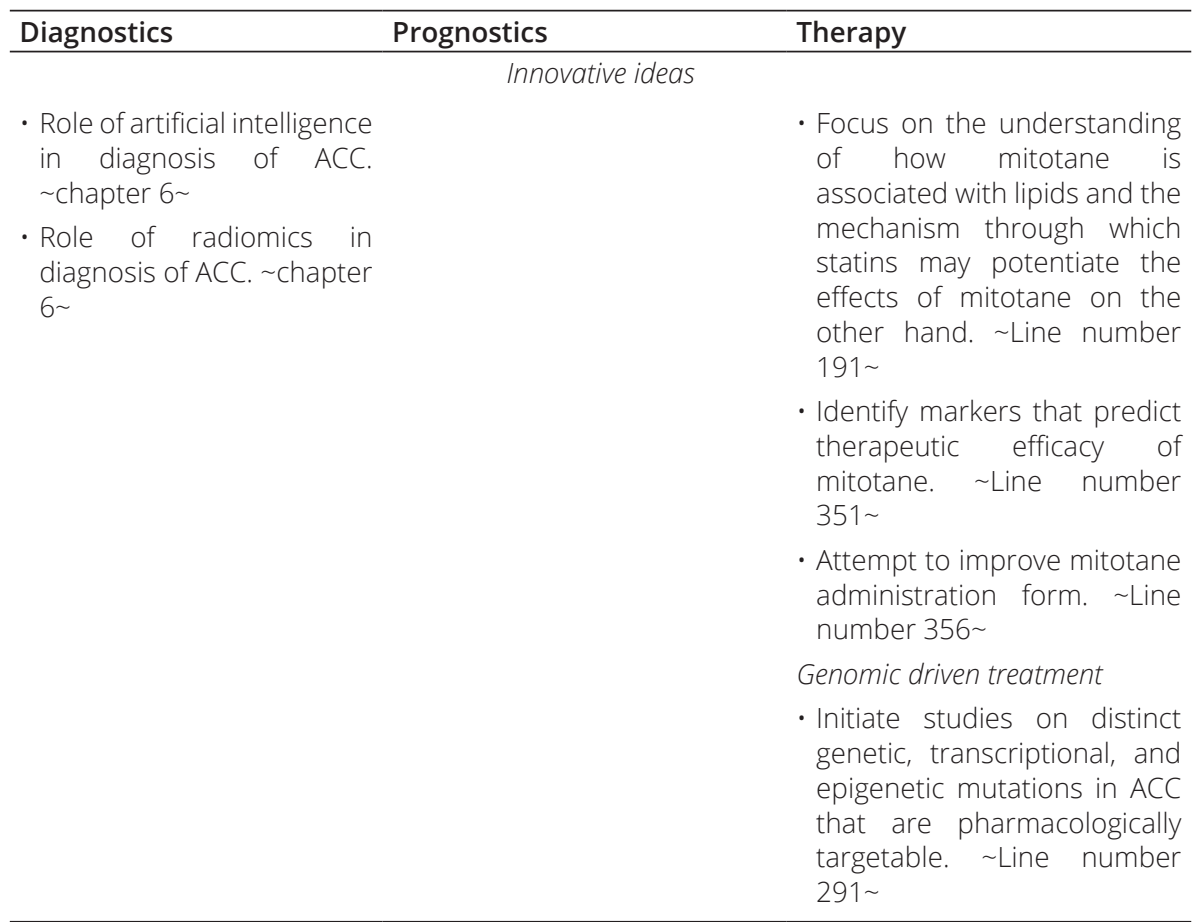

Quality of life Line number 360 - 


\section{References}

1. Datrice NM, Langan RC, Ripley RT, Kemp CD, Steinberg SM, Wood BJ, et al. Operative management for recurrent and metastatic adrenocortical carcinoma. J Surg Oncol 2011:n/a-n/a.

2. op den Winkel J, Pfannschmidt J, Muley T, Grunewald C, Dienemann H, Fassnacht M, et al. Metastatic adrenocortical carcinoma: results of 56 pulmonary metastasectomies in 24 patients. Ann Thorac Surg 2011 Dec;92(6):1965-1970.

3. Prendergast KM, Smith PM, Tran TB, Postlewait LM, Maithel SK, Prescott JD, et al. Features of synchronous versus metachronous metastasectomy in adrenal cortical carcinoma: Analysis from the US adrenocortical carcinoma database. Surgery 2019.

4. Livhits M, Li N, Yeh MW, Harari A. Surgery is associated with improved survival for adrenocortical cancer, even in metastatic disease. Surgery 2014 Dec;156(6):1531-40; discussion 1540-1.

5. Fassnacht M, Dekkers O, Else T, Baudin E, Berruti A, De Krijger RR, et al. European Society of Endocrinology Clinical Practice Guidelines on the management of adrenocortical carcinoma in adults, in collaboration with the European Network for the Study of Adrenal Tumors. European journal of endocrinology 2018;1(aop).

6. Beuschlein F, Weigel J, Saeger W, Kroiss M, Wild V, Daffara F, et al. Major prognostic role of Ki67 in localized adrenocortical carcinoma after complete resection. J Clin Endocrinol Metab 2015 Mar;100(3):841-849.

7. Fassnacht $M$, Johanssen S, Quinkler M, Bucsky P, Willenberg HS, Beuschlein F, et al. Limited prognostic value of the 2004 International Union Against Cancer staging classification for adrenocortical carcinoma: proposal for a Revised TNM Classification. Cancer 2009 01/15;115(0008-543; 0008-543; 2):243-250.

8. Bilimoria KY, Shen WT, Elaraj D, Bentrem DJ, Winchester DJ, Kebebew E, et al. Adrenocortical carcinoma in the United States. Cancer 2008;113(11):3130-3136.

9. Panjwani S, Moore MD, Gray KD, Finnerty BM, Beninato T, Brunaud L, et al. The impact of nodal dissection on staging in adrenocortical carcinoma. Annals of surgical oncology 2017;24(12):3617-3623.

10. Libe R, Borget I, Ronchi CL, Zaggia B, Kroiss M, Kerkhofs T, et al. Prognostic factors in stage III-IV adrenocortical carcinomas (ACC): an European Network for the Study of Adrenal Tumor (ENSAT) study. Ann Oncol 2015 Oct;26(10):2119-2125.

11. Reibetanz J, Jurowich C, Erdogan I, Nies C, Rayes N, Dralle H, et al. Impact of lymphadenectomy on the oncologic outcome of patients with adrenocortical carcinoma. Ann Surg 2012 Feb;255(2):363-369.

12. Gerry JM, Tran TB, Postlewait LM, Maithel SK, Prescott JD, Wang TS, et al. Lymphadenectomy for adrenocortical carcinoma: is there a therapeutic benefit? Annals of surgical oncology 2016;23(5):708-713.

13. Reibetanz J, Rinn B, Kunz AS, Flemming S, Ronchi CL, Kroiss M, et al. Patterns of Lymph Node Recurrence in Adrenocortical Carcinoma: Possible Implications for Primary Surgical Treatment. Annals of surgical oncology 2019;26(2):531-538.

14. Kemp CD, Ripley RT, Mathur A, Steinberg SM, Nguyen DM, Fojo T, et al. Pulmonary resection for metastatic adrenocortical carcinoma: the National Cancer Institute experience. Ann Thorac Surg 2011 Oct;92(4):1195-1200.

15. Kwauk S, Burt M. Pulmonary metastases from adrenal cortical carcinoma: results of resection. J Surg Oncol 1993;53(4):243-246.

16. Asare EA, Wang TS, Winchester DP, Mallin K, Kebebew E, Sturgeon C. A novel staging system for adrenocortical carcinoma better predicts survival in patients with stage I/II disease. Surgery 2014;156(6):1378-1386. 
17. Miller BS, Gauger PG, Hammer GD, Giordano TJ, Doherty GM. Proposal for modification of the ENSAT staging system for adrenocortical carcinoma using tumor grade. Langenbeck's archives of surgery 2010;395(7):955-961

18. Else T, Williams AR, Sabolch A, Jolly S, Miller BS, Hammer GD. Adjuvant therapies and patient and tumor characteristics associated with survival of adult patients with adrenocortical carcinoma. J Clin Endocrinol Metab 2014 Feb;99(2):455-461.

19. Berruti A, Fassnacht M, Haak H, Else T, Baudin E, Sperone P, et al. Prognostic role of overt hypercortisolism in completely operated patients with adrenocortical cancer. Eur Urol 2014 Apr;65(4):832-838

20. Lughezzani G, Sun M, Perrotte P, Jeldres C, Alasker A, Isbarn H, et al. The European Network for the Study of Adrenal Tumors staging system is prognostically superior to the international union against cancer-staging system: a North American validation. Eur J Cancer 2010 Mar;46(4):713-719.

21. Papathomas TG, Pucci E, Giordano TJ, Lu H, Duregon E, Volante M, et al. An International Ki67 Reproducibility Study in Adrenal Cortical Carcinoma. Am J Surg Pathol 2016 Apr;40(4):569-576.

22. Jouinot A, Bertherat J. Management of endocrine disease: adrenocortical carcinoma: differentiating the good from the poor prognosis tumors. European journal of endocrinology 2018;178(5):R215-R230.

23. Dowsett M, Nielsen TO, A'Hern R, Bartlett J, Coombes RC, Cuzick J, et al. Assessment of Ki67 in breast cancer: recommendations from the International Ki67 in Breast Cancer working group. J Natl Cancer Inst 2011;103(22):1656-1664.

24. Lu H, Papathomas TG, van Zessen D, Palli I, de Krijger RR, van der Spek, Peter J, et al. Automated Selection of Hotspots (ASH): enhanced automated segmentation and adaptive step finding for Ki67 hotspot detection in adrenal cortical cancer. Diagnostic pathology 2014;9(1):216.

25. Margonis GA, Kim Y, Tran TB, Postlewait LM, Maithel SK, Wang TS, et al. Outcomes after resection of cortisol-secreting adrenocortical carcinoma. The American Journal of Surgery 2016;211(6):1106-1113.

26. Assie G, Antoni G, Tissier F, Caillou B, Abiven G, Gicquel C, et al. Prognostic parameters of metastatic adrenocortical carcinoma. J Clin Endocrinol Metab 2007 01;92(0021-972; 1):148-154.

27. Icard P, Goudet P, Charpenay C, Andreassian B, Carnaille B, Chapuis Y, et al. Adrenocortical carcinomas: surgical trends and results of a 253-patient series from the French Association of Endocrine Surgeons study group. World J Surg 2001 07;25(0364-2313; 7):891-897.

28. Tran TB, Postlewait LM, Maithel SK, Prescott JD, Wang TS, Glenn J, et al. Actual 10-year survivors following resection of adrenocortical carcinoma. J Surg Oncol 2016;114(8):971-976.

29. Hermsen IG, Gelderblom H, Kievit J, Romijn JA, Haak HR. Extremely long survival in six patients despite recurrent and metastatic adrenal carcinoma. Eur J Endocrinol 2008 06;158(1479-683; 6):911-919.

30. Grisanti S, Cosentini D, Laganà M, Abate A, Rossini E, Sigala S, et al. Are we failing in treatment of AdrenoCortical Carcinoma? Lights and shadows of molecular signatures. Current Opinion in Endocrine and Metabolic Research 2019.

31. Fassnacht M, Berruti A, Baudin E, Demeure MJ, Gilbert J, Haak H, et al. Linsitinib (OSI-906) versus placebo for patients with locally advanced or metastatic adrenocortical carcinoma: a doubleblind, randomised, phase 3 study. Lancet Oncol 2015 Apr;16(4):426-435.

32. Fraenkel M, Gueorguiev M, Barak D, Salmon A, Grossman AB, Gross DJ. Everolimus therapy for progressive adrenocortical cancer. Endocrine 2013 Aug;44(1):187-192.

33. Quinkler M, Hahner S, Wortmann S, Johanssen S, Adam P, Ritter C, et al. Treatment of advanced adrenocortical carcinoma with erlotinib plus gemcitabine. J Clin Endocrinol Metab 2008 Jun;93(6):2057-2062. 
34. Samnotra V, Vassilopoulou-Sellin R, Fojo AT, Oh WK, LaRocca RV, Ernstoff MS, et al. A phase II trial of gefitinib monotherapy in patients with unresectable adrenocortical carcinoma (ACC). 2007;25(18S).

35. Haak HR, Hermans J, van de Velde CJ, Lentjes EG, Goslings BM, Fleuren GJ, et al. Optimal treatment of adrenocortical carcinoma with mitotane: results in a consecutive series of 96 patients. $\mathrm{Br}$ J Cancer 1994 05;69(0007-0920; 5):947-951.

36. Baudin E, Pellegriti G, Bonnay M, Penfornis A, Laplanche A, Vassal G, et al. Impact of monitoring plasma 1,1-dichlorodiphenildichloroethane (o,p'DDD) levels on the treatment of patients with adrenocortical carcinoma. Cancer 2001 09/15;92(0008-543; 6):1385-1392.

37. van Slooten $H$, Moolenaar AJ, van Seters AP, Smeenk D. The treatment of adrenocortical carcinoma with o,p'-DDD: prognostic implications of serum level monitoring. Eur J Cancer Clin Oncol 1984 01;20(0277-5379; 1):47-53.

38. Moy RH. Studies of the pharmacology of o,p'DDD in man. J Lab Clin Med 1961 08;58(0022-2143; 0022-2143):296-304.

39. Moolenaar AJ, van SH, van Seters AP, Smeenk D. Blood levels of o,p'-DDD following administration in various vehicles after a single dose and during long-term treatment. Cancer Chemother Pharmacol 1981;7(0344-5704; 1):51-54.

40. von Slooten H, van Seters AP, Smeenk D, Moolenaar AJ. O,p'-DDD (mitotane) levels in plasma and tissues during chemotherapy and at autopsy. Cancer Chemother Pharmacol 1982;9(2):85-88

41. Hermansson V, Cantillana T, Hovander L, Bergman A, Ljungvall K, Magnusson U, et al. Pharmacokinetics of the adrenocorticolytic compounds 3-methylsulphonyl-DDE and o,p'-DDD (mitotane) in Minipigs. Cancer Chemother Pharmacol 2008 Feb;61(2):267-274.

42. Kerkhofs TM, Derijks LJ, Ettaieb H, den Hartigh J, Neef K, Gelderblom H, et al. Development of a pharmacokinetic model of mitotane: toward personalized dosing in adrenocortical carcinoma. Ther Drug Monit 2015 Feb;37(1):58-65.

43. Mornar A, Sertic M, Turk N, Nigovic B, Korsic M. Simultaneous analysis of mitotane and its main metabolites in human blood and urine samples by SPE-HPLC technique. Biomed Chromatogr 2012 Nov;26(11):1308-1314.

44. D'Avolio A, De Francia S, Basile V, Cusato J, De Martino F, Pirro E, et al. Influence of the CYP2B6 polymorphism on the pharmacokinetics of mitotane. Pharmacogenet Genomics 2013 Mar 20.

45. van Erp NP, Guchelaar HJ, Ploeger BA, Romijn JA, Hartigh J, Gelderblom H. Mitotane has a strong and a durable inducing effect on CYP3A4 activity. Eur J Endocrinol 2011 Apr;164(4):621-626.

46. Medina-Diaz IM, Arteaga-Illan G, de Leon MB, Cisneros B, Sierra-Santoyo A, Vega L, et al. Pregnane $X$ receptor-dependent induction of the CYP3A4 gene by o,p'-1,1,1,-trichloro-2,2-bis (p-chlorophenyl)ethane. Drug Metab Dispos 2007 Jan;35(1):95-102.

47. Medina-Diaz IM, Elizondo G. Transcriptional induction of CYP3A4 by o, p'-DDT in HepG2 cells. Toxicol Lett 2005;157(1):41-47.

48. Kroiss M, Quinkler M, Lutz WK, Allolio B, Fassnacht M. Drug interactions with mitotane by induction of CYP3A4 metabolism in the clinical management of adrenocortical carcinoma. Clin Endocrinol (Oxf) 2011 Nov; 75(5):585-591.

49. Gross DJ, Munter G, Bitan M, Siegal T, Gabizon A, Weitzen R, et al. The role of imatinib mesylate (Glivec) for treatment of patients with malignant endocrine tumors positive for c-kit or PDGF-R. Endocr Relat Cancer 2006 Jun; 13(2):535-540.

50. Hescot S, Seck A, Guerin M, Cockenpot F, Huby T, Broutin S, et al. Lipoprotein-free mitotane exerts high cytotoxic activity in adrenocortical carcinoma. The Journal of Clinical Endocrinology \& Metabolism 2015;100(8):2890-2898. 
51. Boulate G, Amazit L, Naman A, Seck A, Paci A, Lombes A, et al. Potentiation of mitotane action by rosuvastatin: New insights for adrenocortical carcinoma management. Int J Oncol 2019;54(6):2149-2156.

52. Shawa H, Deniz F, Bazerbashi H, Hernandez M, Vassilopoulou-Sellin R, Jimenez C, et al. Mitotaneinduced hyperlipidemia: a retrospective cohort study. Int J Endocrinol 2013;2013:624962.

53. Severe hypertriglyceridemia in relation to toxic levels of mitotane in a patient with stage IV adrenocortical carcinoma (ACC). 19th European Congress of Endocrinology: BioScientifica; 2017.

54. Paci A, Hescot S, Seck A, Jublanc C, Mercier L, Vezzosi D, et al. Dyslipidemia causes overestimation of plasma mitotane measurements. Endocrinology, diabetes \& metabolism case reports 2016;2016(1).

55. Wortsman J, Soler NG. Mitotane. Spironolactone antagonism in Cushing's syndrome. JAMA 1977 Dec 5;238(23):2527.

56. Blume $H$, Donath F, Warnke A, Schug BS. Pharmacokinetic drug interaction profiles of proton pump inhibitors. Drug safety 2006;29(9):769-784.

57. Kroiss M, Sbiera S, Kendl S, Kurlbaum M, Fassnacht M. Drug synergism of proteasome inhibitors and mitotane by complementary activation of ER stress in adrenocortical carcinoma cells. Hormones and Cancer 2016;7(5-6):345-355.

58. Kerkhofs TM, Baudin E, Terzolo M, Allolio B, Chadarevian R, Mueller HH, et al. Comparison of two mitotane starting dose regimens in patients with advanced adrenocortical carcinoma. J Clin Endocrinol Metab 2013 Dec;98(12):4759-4767.

59. Di Paolo A, Ciofi L, Bacca A, Bernini G. A case report of a TDM-guided optimization of mitotane for a safe and effective long-term treatment. Journal of Chemotherapy 2019;31(2):105-108.

60. Mauclere-Denost S, Leboulleux S, Borget I, Paci A, Young J, Al Ghuzlan A, et al. High-dose mitotane strategy in adrenocortical carcinoma: prospective analysis of plasma mitotane measurement during the first 3 months of follow-up. Eur J Endocrinol 2012 Feb;166(2):261-268.

61. Terzolo M, Pia A, Berruti A, Osella G, Ali A, Carbone V, et al. Low-dose monitored mitotane treatment achieves the therapeutic range with manageable side effects in patients with adrenocortical cancer. J Clin Endocrinol Metab 2000 06;85(0021-972; 6):2234-2238.

62. Barreau O, Assie G, Wilmot-Roussel H, Ragazzon B, Baudry C, Perlemoine K, et al. Identification of a CpG island methylator phenotype in adrenocortical carcinomas. J Clin Endocrinol Metab 2013 Jan;98(1):E174-84.

63. Assie G, Letouze E, Fassnacht M, Jouinot A, Luscap W, Barreau O, et al. Integrated genomic characterization of adrenocortical carcinoma. Nat Genet 2014 Jun;46(6):607-612.

64. Zheng S, Cherniack AD, Dewal N, Moffitt RA, Danilova L, Murray BA, et al. Comprehensive pangenomic characterization of adrenocortical carcinoma. Cancer cell 2016;29(5):723-736.

65. Mohan DR, Lerario AM, Else T, Mukherjee B, Almeida MQ, Vinco M, et al. Targeted assessment of G0S2 methylation identifies a rapidly recurrent, routinely fatal molecular subtype of adrenocortical carcinoma. Clinical Cancer Research 2019;25(11):3276-3288.

66. Fragoso MCB, Almeida MQ, Mazzuco TL, Mariani BM, Brito LP, Gonçalves TC, et al. Combined expression of BUB1B, DLGAP5, and PINK1 as predictors of poor outcome in adrenocortical tumors: validation in a Brazilian cohort of adult and pediatric patients. European journal of endocrinology 2012;166(1):61.

67. Takai D, Jones PA. Comprehensive analysis of CpG islands in human chromosomes 21 and 22. Proc Natl Acad Sci U S A 2002 Mar 19;99(6):3740-3745.

68. Koch A, Joosten SC, Feng Z, de Ruijter TC, Draht MX, Melotte V, et al. Analysis of DNA methylation in cancer: location revisited. Nature Reviews Clinical Oncology 2018;15(7):459-466. 
69. De Reynies A, Assie G, Rickman DS, Tissier F, Groussin L, Rene-Corail F, et al. Gene expression profiling reveals a new classification of adrenocortical tumors and identifies molecular predictors of malignancy and survival. J Clin Oncol 2009 03/01;27(1527-7755; 7):1108-1115.

70. Sbiera S, Leich E, Liebisch G, Sbiera I, Schirbel A, Wiemer L, et al. Mitotane inhibits sterol-Oacyl transferase 1 triggering lipid-mediated endoplasmic reticulum stress and apoptosis in adrenocortical carcinoma cells. Endocrinology 2015;156(11):3895-3908.

71. Crona J, Beuschlein F. Adrenocortical carcinoma - Towards genomics guided clinical care. Nature Reviews Endocrinology 2019;15(9):548-560.

72. Ross JS, Wang K, Rand JV, Gay L, Presta MJ, Sheehan CE, et al. Next-generation sequencing of adrenocortical carcinoma reveals new routes to targeted therapies. J Clin Pathol 2014 Nov;67(11):968-973.

73. Fiorentini C, Fragni M, Tiberio GA, Galli D, Roca E, Salvi V, et al. Palbociclib inhibits proliferation of human adrenocortical tumor cells. Endocrine 2018;59(1):213-217.

74. LoRusso PM, Rudin CM, Reddy JC, Tibes R, Weiss GJ, Borad MJ, et al. Phase I trial of hedgehog pathway inhibitor vismodegib (GDC-0449) in patients with refractory, locally advanced or metastatic solid tumors. Clin Cancer Res 2011 Apr 15;17(8):2502-2511. 


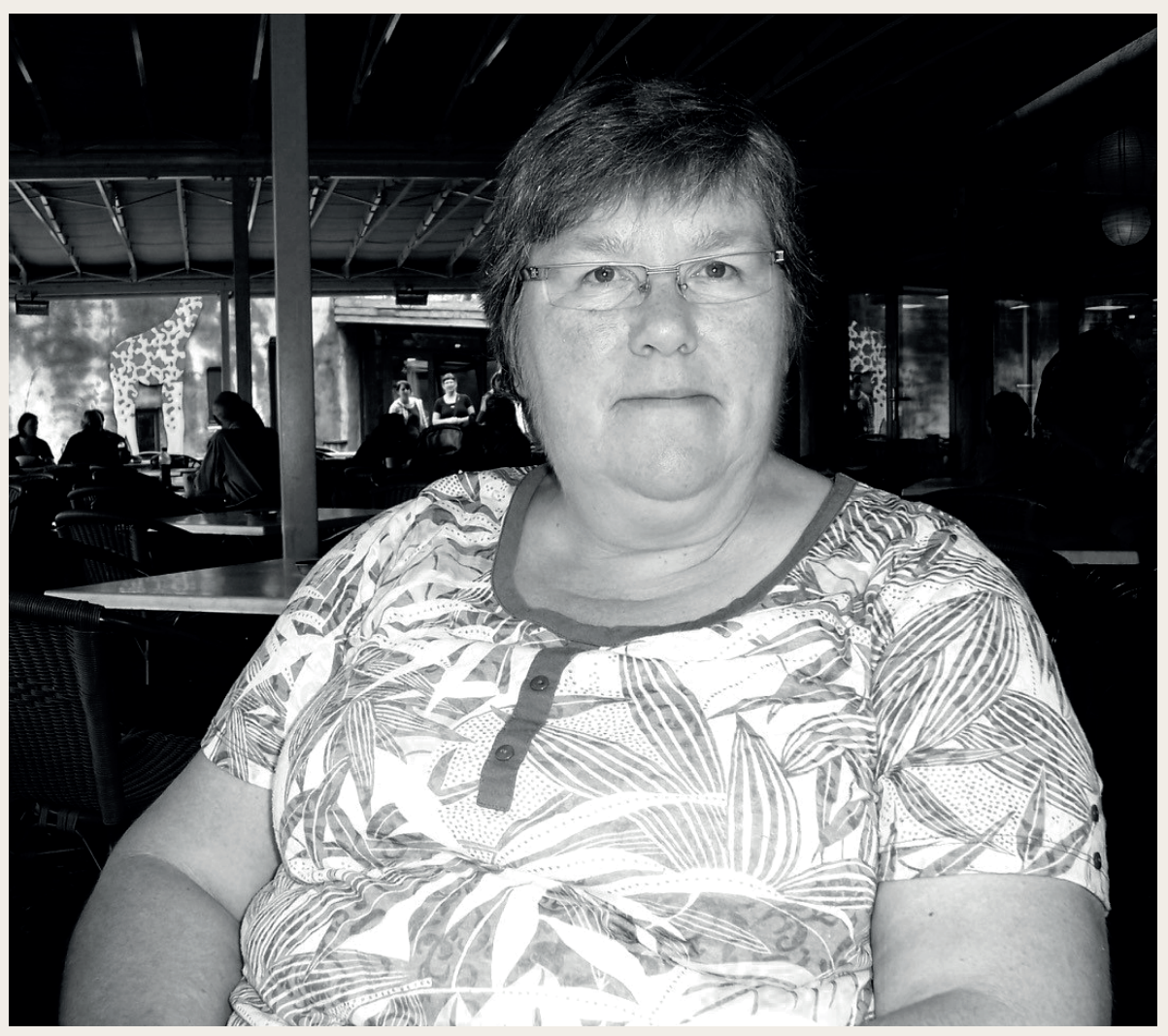


Chapter 08

Summary 


\section{Summary}

The present thesis was designed to investigate adrenocortical carcinoma (ACC) from a clinical perspective with focus on survival.

ACC is a rare but aggressive malignancy. Unfortunately, most patients present with advanced disease at presentation. Chapter 2 presents a retrospective populationbased study with the focus on the time and extent of metastasis. Patients who presented with ACC between January 1, 2004 to October 31, 2013 in one of the nine centers of the Dutch Adrenal Network (DAN) were included. The total number of ACC patients was 160. One hundred seventeen patients (73\%) were diagnosed with metastases during the follow-up period of this study: eighty-four patients had synchronous metastases and thirty-three developed metachronous metastases. Overall survival (OS) rate was calculated from the date of diagnosis of metastasis until death from any cause.

Median OS after diagnosis of first metastases for patients with synchronous or metachronous metastases was 12 months and 29 months, respectively, ( $p=0.046)$.

Forty patients (34\%) had one to three metastases. The other 77 patients (66\%) had four or more metastases. Overall survival for these two groups was significantly different with $50 \%$ and $12 \%$ respectively $(p<0.001)$.

Furthermore, the number of affected organs was of influence on median survival. Seventy-five patients had only one affected organ at presentation, in 42 patients two or more organs were involved: OS percentages were $33 \%$ and $10 \%$ respectively $(\mathrm{p}<0.001)$.

ACC prognosis is heterogeneous. European Network for the Study of Adrenal Tumors (ENSAT) stage is the most used prognosticator, advised in the guidelines. In chapter 3 a prediction model for ACC-specific mortality is developed. One hundred fifty-nine patients from the DAN database were selected for this prospective cohort study.

Internal validation of the model was performed using standard bootstrapping techniques.

The multivariable modeling yielded a prediction model which included age, modified European Network for the Study of Adrenal Tumors (mENSAT) stage, and radical resection. The c-statistic was 0.77 (95\% Confidence Interval: 0.72, 0.81), indicating good predictive performance of the model.

Internal validation proofed the model not be over fitted. Applicability of the model is illustrated by calculating the two-year probability of ACC-specific mortality of a 64 year old patient with an mENSAT stage of $4 a$, and no radical resection of the tumor: $1-0.56 \exp (L P) . L P=0.02$ * $(64-54.5)+0.63 *(4-3.2)-0.44$ * $(0-0.8)=1.046$; hence, the probability $=1-0.56 \exp (1.046)=0.81$, being $81 \%$. 
Mitotane is the adrenolytic drug recommended for treatment of primary and recurrent adrenocortical carcinoma. Mitotane exerts an anti-neoplastic effect on ACC tissue and inhibits cortisol synthesis. Ultimately, mitotane efficacy is limited by its high lipophilicity, poor pharmacokinetic properties, and dose-limiting toxicities. A plasma level between $14-20 \mathrm{mg} / \mathrm{I}$ is considered as therapeutic range. Reaching and maintaining these therapeutic levels is complicated and takes months. In chapter 4 a population pharmacokinetic (PK) and pharmacogenetic analysis was performed for mitotane in patients with ACC utilizing the retrospectively collected PK data on 48 patients. The effect of genes encoding drug absorption, distribution, metabolism, and elimination (ADME), patients' demographic information, and clinical characteristics on mitotane PK were investigated as covariates.

A two-compartment model with first-order absorption and elimination best described the PK data of mitotane. Lean body weight (LBW), genotypes of CYP2C19*2 (rs4244285), SLCO1B3 699A>G (rs7311358), and SLCO1B1 571T>C (rs4149057) were identified to affect mitotane clearance (CL/F) significantly, which decreased the coefficient of variation (CV \%) of random inter-individual variability of CL/F from $67.0 \%$ to $43.0 \%$. Fat amount (i.e. body weight - LBW) was identified to affect the central distribution volume significantly. The developed model is beneficial to optimize mitotane treatment schedules and to guide the initial dose selection for patients.

Chapter 5 contains a review with perspectives on the future of ACC treatment. When optimizing the diagnostic and therapeutic process in ACC care, minimum volume requirements as instituted for other cancers are hardly feasible due to the low annual incidence of 0.5 -2.0 patient per million people per year. However, we advocate the establishment of an international 'standard of care' that defines mandatory elements in clinical management of ACC patients. Uniformity can be achieved by defining in detail the entire process a patient undergoes from the first diagnostic test until follow-up visits. A summary of key points that should be present in an ACC-specialized center are presented. This comprises for example timing of diagnostic evaluations, standardized formats of pathology and surgery reports, guidelines for (supportive) therapy, etcetera. In addition, participation in international networks and clinical trials should be encouraged.

The understanding of adrenocortical tumorigenesis has improved over the years and the last decade studies have been focusing on epigenetic changes associated with adrenocortical tumors. In chapter 6 a descriptive review provides an overview on the evolving field of epigenetic studies on adrenal tumors. The level of methylation in adrenal carcinoma has been found to be associated with survival. The level of CpG island promoter methylation, or CIMP, has been shown in various studies to provide a classification based on methylation as a marker for prognosis in patients with ACC. Actually, we are on the verge of the development of a biomarker for prognosis in 
ACC. GOS2 hypermethylation was shown to be a hallmark of the CIMP-high cluster, associated with poor outcome. The establishment of a reproducible biomarker, will have implications for the diagnostic and prognostic process of ACC in the near future. 


\section{Nederlandse samenvatting}

Dit proefschrift richt zich op onderzoek van kanker van de buitenste laag, het schors, van de bijnier: het bijnierschorscarcinoom. De bijnieren zijn hormoon producerende organen die bij de nieren liggen. Bijnierschorscarcinoom is een zeldzame vorm van kanker met een slechte prognose.

In Nederland is er een samenwerkingsverband van medisch specialisten, het Bijnier Netwerk Nederland (BNN), dat zich inspant om patiënten met bijnierkanker een zo goed mogelijke behandeling te geven. Dit is een samenwerking van alle Universitair Academisch Medische Centra en het Máxima Medisch Centrum.

Tot 30 procent van de patiënten met bijnierschorskanker, presenteren zich met reeds uitgezaaide (niet meer te genezen) ziekte, stadium IV kanker. In hoofdstuk 2 wordt een studie beschreven waar wordt gekeken of het moment van ontwikkelen van uitzaaiingen (metastasen), van invloed is op de overleving. Patiënten van 18 jaar of ouder, die zich tussen 1 januari 2004 en 31 oktober 2013 presenteerden in een van de centra van het BNN, werden geïncludeerd in de studie. Patiënten die tot 6 maanden na primaire diagnose metastasen kregen de definitie synchroon gemetastaseerd. Patiënten die pas metastasen kregen vanaf 6 maanden na de primaire diagnose kregen de definitie metachroon gediagnostiseerd. Er werden 160 patiënten in het onderzoek geïncludeerd. Honderd-zeventien patiënten werden geconfronteerd met metastasen. Vierentachtig patiënten hadden synchroon gemetastaseerd bijnierschorskanker en 33 metachroon. Patiënten met metachroon gemetastaseerde ziekte hadden een betere overleving dan patiënten met synchroon gemetastaseerde ziekte.

Tevens werd er gekeken naar de invloed van het aantal metastasen en aantal organen waarin uitzaaiingen aanwezig waren, op de overleving. Er werd geobserveerd dat patiënten met drie of meer metastasen en twee of meer organen met metastasen een slechtere prognose hadden.

De overlevingsduur voor bijnierschorskanker patiënten is globaal genomen somber. Een stadiëring systeem wordt gebruikt om op een generaliseerbare manier de grootte en de graad van verspreiding van de kanker te beschrijven. Bij bijnierschorskanker is dit het Europese netwerk voor studies naar bijniertumoren (European Network for the Study of Adrenal Tumors; ENSAT) systeem. Aan de hand van dit systeem kan een voorspelling worden gedaan over de kans op overleven.

Als er wordt gekeken naar de overleving van bijnierschorskanker patiënten binnen de verschillende ENSAT stadia is er heterogeniteit en er is dan ook behoefte aan een methode, dan wel stadiering, waarmee een meer nauwkeurige inschatting over de prognose kan worden gemaakt. In hoofdstuk $\mathbf{3}$ wordt een voorspellend model beschreven waarmee het mogelijk is de kans op overlijden aan (de gevolgen van) 
bijnierschorskanker voor een individuele patiënt te berekenen. Het model bestaat uit een aantal voorspellende factoren (predictoren).

In 2015 is door een onderzoeksgroep in Frankrijk een suggestie voor een aanpassing op de ENSAT stadiering gepubliceerd: mENSAT. Patiënten met betrokkenheid van lymfeklieren worden in dit stadiëring systeem als stadium IV, in plaats van stadium III, beschouwd. Tevens zijn de stadium IV patiënten verdeeld in sub stadia a-c afhankelijk van het aantal organen met uitzaaiingen (2, 3 of $>3$ ) waarbij lymfeklieren als orgaan worden gerekend en ook de primaire tumor als aangetast orgaan wordt meegerekend.

Het in hoofdstuk 3 gepresenteerde model is in staat om met goede accuraatheid de kans op mortaliteit te berekenen voor een individuele patiënt aan de hand van leeftijd ( $\geq 18$ jaar), mENSAT stadium en in hoeverre de kanker radicaal is verwijderd door de chirurg.

De resultaten uit hoofdstuk 2 en hoofdstuk $\mathbf{3}$ van dit proefschrift bemoedigen de implementatie van mENSAT. Het aantal aangedane organen speelt een rol in de prognose. Dit zien we ook terug in mENSAT. Een grootschalig prospectief onderzoek wat ENSAT en mENSAT tegen elkaar uitzet in hun vermogen prognose van de patiënt te voorspellen, kan extra bewijs leveren mENSAT op te nemen in de richtlijn.

Tevens wordt gesuggereerd dat patiënten met lymfeklier en orgaan metastasen wellicht extra aandacht verdienen van de behandelaren, waar er heterogeniteit is in overleving. Dit vereist maatwerk in de behandeling van een patiënt met uitzaaiingen. Aanvullend onderzoek is nodig om patiënten met metastasen doch een relatief gunstige prognose beter te kunnen detecteren zodat de primaire behandeling zich daar op aanpast en dat bijvoorbeeld de chirurg wordt gevraagd zo veel mogelijk afwijkingen te verwijderen.

De enige curatieve optie bij niet-uitgezaaid bijnierschorscarcinoom is chirurgische resectie van de primaire tumor. Echter, zelfs nadat de tumor volledig is weggesneden is er een kans op terugkeren van de kanker. Medicamenteuze opties zijn beperkt. Hierin speelt het medicijn mitotaan een hoofdrol. Dit medicijn kent echter ernstige bijwerkingen en wordt de therapie door patiënten als invaliderend ervaren.

Mitotaan vernietigt vooral bijnierschorsweefsel en wordt al langer gebruikt bij patiënten met gemetastaseerde ziekte. Onderzoek heeft aangetoond dat behandeling met mitotaan na complete resectie van de tumor de ziektevrije overleving verlengt. Over het exacte werkingsmechanisme van mitotaan is onvoldoende bekend. Wel zijn er sterke aanwijzingen dat het therapeutisch effect afhankelijk is van de plasmaconcentratie in het bloed: een plasmaspiegel van 14 milligram per liter wordt algemeen aangehouden als ondergrens voor werkzaamheid. Plasmaspiegels boven de $20 \mathrm{mg} / \mathrm{L}$ zijn geassocieerd met toxiciteit die belastend kan zijn voor de patiënt. Dit smalle therapeutisch venster maakt nauwkeurige dosering en monitoring 
noodzakelijk. Echter, de optimale opbouw- en onderhoudsdosering blijkt onder patiënten sterk te verschillen, wat het doseren van mitotaan danig bemoeilijkt. In de praktijk heeft dit tot gevolg dat er bij bepaalde patiënten sprake is van onder-dosering, wat ongunstig is omdat er dan geen effectieve behandeling van de tumor plaatsvindt, of juist overdosering waarbij er neurotoxische of gastro-intestinale bijwerkingen kunnen optreden.

In hoofdstuk 4 is er specifiek gekeken naar DNA van genen betrokken bij de absorptie, distributie, activering en excretie van geneesmiddelen van patiënten met bijnierschorskanker die behandeld zijn met mitotaan. Aan de hand van de patiënt eigenschappen en hun DNA, is een model ontwikkeld waarmee de optimale mitotaan dosering kan worden berekend om tot een therapeutische spiegel te komen. De klaring van mitotaan wordt significant beïnvloedt door de vetvrije massa en de genen (erfelijk materiaal) met het volgende genotype: CYP2C19*2 (rs4244285), SLCO1B3 699A>G (rs7311358) en SLC01B1 571T>C (rs4149057).

In Hoofdstuk 5 van dit proefschrift wordt een oproep gedaan om de zorg voor bijnierschorskankerpatiënten te verbeteren. Het hoofdstuk biedt een overzicht van de diagnostiek, voorspellende factoren en behandeling van bijnierschorskanker. Er wordt een suggestie gedaan voor een uniforme benadering die moet waarborgen dat de zorg volgens een bepaalde standaard wordt geleverd. Belangrijke onderdelen van deze uniforme zorg is onder andere een standaard richtlijn voor de frequentie van de controles, een standaard format voor het operatieverslag en pathologieverslag. Daarnaast is het ontzettend belangrijk om nationaal en internationaal te blijven samenwerken om met een groter aantal patiënten participatie onderzoek te kunnen doen.

Er is dringend behoefte aan aanvullende behandelingen om de overleving van bijnierschorskanker te verbeteren.

Om dit te kunnen bewerkstelligen is met name begrip van de pathofysiologie van de ziekte van belang. Waarom en hoe ontstaat het bijnierschorscarcinoom?

Kanker kunnen we slechts gedeeltelijk begrijpen als we alleen in het DNA naar de genen kijken. Bij het epigenetisch onderzoek kijken we naar niet coderende genen die onder druk van omgevingsfactoren mee bepalen of een coderend gen 'aan' of 'uit' wordt gezet en of een gen dus wel of niet wordt afgelezen. Op je DNA zitten moleculaire 'dimmers' die regelen of genen 'aan' of 'uit' kunnen staan. Een voorbeeld van moleculaire 'dimmers' warover we al veel weten, zijn de zogeheten methylgroepen. Zijn er veel methylmoleculen aan genen gekoppeld, dan zijn deze genen uitgeschakeld; zijn er weinig methylmoleculen, dan kunnen de genen actief worden. Zo een cluster van 'dimmers' kom je veel tegen rond een speciaal gebied in het DNA, het zogenaamde promotor gebied. De promotor is de startknop voor het lezen van je DNA. 
Indien we zulke gebieden op de genen bij bijnierkanker die overmatig 'aan' of 'uit' staan kunnen identificeren dan verschaft dit niet alleen meer inzicht in het ontstaan van de ziekte maar het kan ook leiden tot de ontwikkeling van nieuwe therapieën. Hoofdstuk 6 is een review artikel bestaand uit een groot literatuuronderzoek. Het geeft een samenvatting van de stand van zaken op het gebied van epigenetica van bijnierschorskanker. De verwachting is dat methyl patronen op korte termijn een belangrijke rol gaan spelen in de voorspelling van overleving van bijnierschorskanker waar deze patronen een associatie tonen met mate van agressiviteit van de kanker.

Hoofdstuk 7 bevat een algemene discussie waarin de bevindingen uit dit proefschrift worden bediscussieerd in een breder perspectief en waarin suggesties voor toekomstig onderzoek worden gedaan. 
8 


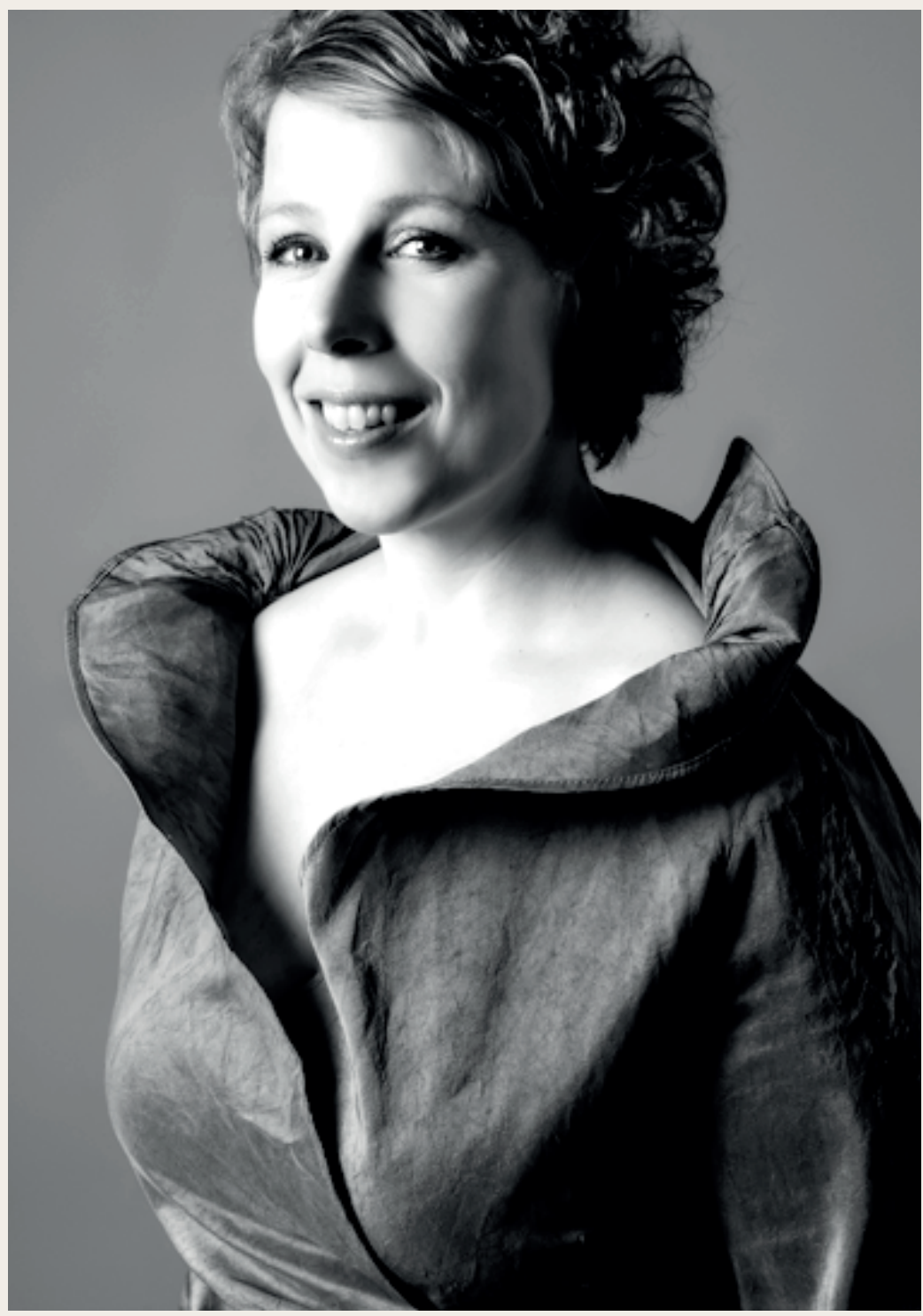


Chapter 09

Impact paragraph 
Adrenocortical carcinoma (ACC) is a rare type of cancer. This thesis focuses on the identification of markers that could improve the accuracy of diagnosing, treating, and estimating survival of ACC.

\section{Survival}

In chapter 2 of this thesis it is concluded that patients who develop metastases within six months after initial diagnosis of ACC have a more poor overall survival (OS) than patients with metachronous metastasis. Also, patients with a high disease burden at diagnosis, four or more metastases or two or more affected organs, have a more grim overall survival than patients with limited disease. Furthermore, it is suggested that surgery might have a positive impact on OS for patient with synchronous metastases. In chapter 3 a prediction model for ACC specific mortality is proposed. The model is based on age, modified European Network for the Study of Adrenal Tumors (mENSAT) stage, and radical resection.

ACC has a heterogeneous prognosis. Studies have been published describing aggressive stage III ACC, biologically behaving like stage IV, but also stage IV patients with exceptional long survival. This emphasizes that the biological heterogeneous behavior of ACC is not fully captioned with ENSAT stage. Therefore, there is a need for better prognostication in ACC to inform the individual patient on survival and assist in deciding on treatment strategy. The impact of chapter $\mathbf{2}$ and $\mathbf{3}$ is the addition of information on ACC prognosis, to better capture the biologically heterogeneous behavior of ACC, not fully endorsed by the ENSAT stage. We hope that this new information will support the implementation of mENSAT in the ACC guideline. Better staging may encourage earlier, for example, surgical intervention through defining of (severe) prognosis, and may support further research into surgery in advanced ACC.

The information on prognostication is relevant for both ACC patients as well as their caregivers. Our research has been published and has been presented at national and international congresses.

If, in the future, our study outcomes will be implemented in daily practice it would be relevant to inform patients, for example using online platforms (international Facebook patient groups, accsupport.org.uk etc.).

\section{Treatment}

Mitotane is a keystone in the treatment of ACC. It is used both in adjuvant setting as well as in patients with metastatic disease. Mitotane has a small therapeutic window of 14-20 milligram per liter. Survival benefit has been proven in patients with blood 
levels higher than $14 \mathrm{mg} / \mathrm{L}$ whereas blood levels higher than $20 \mathrm{mg} / \mathrm{L}$ are associated with increased toxicity. Toxicity is primarily gastro-intestinal and neurological and even leads to temporary or final discontinuation of mitotane therapy in some cases.

There is a large inter-patient variability in reaching and maintaining therapeutic concentrations, without a clear relationship between the mitotane dose and the serum concentration. The build-up of the therapeutic plasma concentration is in general slow. In most patients this level is reached after three months of treatment with a daily dose of about 6.0 gram (12 tablets of 500 milligrams). This means a delay of the optimal therapeutic effect. This complicates timing of therapy evaluation and consequently, the decision to add cytotoxic chemotherapy to the treatment.

Dosing regimens are based on clinical experience and adjusted according to plasma concentration and tolerability. The inability to predict mitotane levels leads to relative under dosing and a prolonged build-up phase in some patients, while others unexpectedly demonstrate high plasma levels early in therapy, causing increased toxicity. Earlier it was shown that there are only weak correlations between weight, age, gender, height and the pharmacokinetics of mitotane. The main goal in chapter 4 was identifying pharmacogenetic differences among patients treated with mitotane by analyzing genes related involved in drug absorption, distribution, metabolism and elimination (ADME). In chapter 4 it is shown that pharmacokinetics of mitotane can partially be explained by pharmacogenetics: a two compartment pharmacologic model is presented best describing the population pharmacokinetic (PK) data of mitotane. Lean body weight (LBW), genotypes of CYP2C19*2 (rs4244285), SLCO1B3 699A $>\mathrm{G}$ (rs7311358), and SLCO1B1 571T>C (rs4149057) were identified to affect mitotane clearance (CL/F) significantly.

The developed model is beneficial to optimize mitotane treatment schedules and to guide the initial dose selection for patients. Therefore it is relevant for ACC patients starting with mitotane therapy.

Currently only half of the patients treated with mitotane reach therapeutic levels and up to $50 \%$ of patients discontinue treatment because of toxicity. Our pharmacokinetic model could assist optimization of mitotane dosage to guide the build up to a therapeutic level, but prevent overshooting to toxic levels.

The model needs to be externally validated: it needs to be tested with a different set of patients to see if the results of our study can be generalized to and across other patients in different contexts.

If validated we would encourage the use of an online resource to make our model available worldwide for experts who treat ACC patients. The shiny app could for example be implemented in the ENSAT website. 


\section{Orphan disease}

Adrenocortical carcinoma is an orphan disease. There are multiple definitions of orphan disease: it has been used to describe diseases that are neglected by doctors, or used to designate diseases that affect only small numbers of individuals and as disease that has not been adopted by the pharmaceutical industry because it provides little financial incentive for the private sector to make and market new medications to treat or prevent it. It defines a large group of diseases which are characterized by a low prevalence in the population. They frequently are associated with problems in diagnosis and treatment.

In chapter 5 foreseen changes in diagnostic and prognostic instruments are discussed and suggestions are made for clinical management and possibilities for future treatment of ACC.

It has been proven repeatedly that the establishment of (inter)national collaborative networks of expert centers has a favorable effect on survival of ACC patients. Because of ACC being an orphan disease, we suggest that quality criteria other than volume criteria might be of greater importance: it seems more important that centers adhere to treatment as suggested in up to date guidelines, which in turn seems best feasible in a specialized centers with dedicated physicians. Furthermore, these international collaborative networks, such as ENSAT, encourage clinical trials. By working together sufficient patient numbers can be provided in order to achieve adequate reductions in uncertainties about e.g. treatment effects and run those clinical trials to test new treatment possibilities that are so badly needed. The impact of chapter $\mathbf{5}$ is that it goes against current ideology of treating cancer patients in high-volume facilities. It endorses the treatment of ACC with mitotane by experts, even if this means treatment in low volume centers. It encourages initiatives as the European Network for the Study of Adrenal Tumors (ENSAT) and the A5: American-Australian-Asian Adrenal Alliance. It is a plea for collaboration even in the current competitive scientific culture where the credo seems to be 'to publish or perish'.

Many of the suggestions made in chapter 5 (additional prognostic factors are needed; a uniform methodology for assessing the Ki-67 index in ACC needs to be established; centers should adhere to current state-of-art treatment concepts which seems best feasible in a specialized centers with dedicated physicians, adequate supportive treatment during mitotane therapy cannot be stressed enough; international collaboration is necessary to facilitate fundamental research and clinical trials to test new treatment possibilities) are included in the European guideline published in 2018, for example the recommendation that the pathology report should at least contain Weiss score (including the exact mitotic count), exact Ki67 index, resection status and pathological tumor stage (indicating invasion or not of the capsule and/or surrounding tissue and organs) and nodal status. 
Also, chapter 5 has been cited for at least 14 times by other scientific authors.

Epigenetics

Adrenocortical carcinoma occurs both as an inherited form of cancer as well as a sporadic neoplasm, being prominent in the Brazilian population.

Inherited ACC, associated with familiar syndromes, as well as the increased incidence in the Brazilian population is associated with a mutation in TP53, a gene that codes for a protein that regulates the cell cycle and hence functions as a tumor suppression. In sporadic ACC a TP53 mutation is often lacking.

Overall, the understanding of the pathophysiology, especially genetic cause, of ACC is limited. Mutations in insulin-like growth factor 2 (IGF2), $\beta$-catenin (CTNNB1 or ZNRF3), and TP53 have been associated with ACC. So, if the usual mutation analysis doesn't explain the familial cause, how can we solve the conundrum of how ACC develops? The answer might be epigenetics.

Epigenetic alterations occur in cancer cells as commonly as genetic mutations and have the ability to mimic the effects of the latter. Therefore, epigenetics are currently an increasing interest in the subject of cancer research. Epigenetics refers to the non-sequence-based modifications of DNA or its associated factors (e.g., histones) that are maintained during cell division. Most epigenetic changes concern DNA methylation, attaching methyl groups to segments of DNA, and histone modification. Hypermethylation is a common phenomenon seen in cancer. Studies suggests that epigenetic alterations precede tumor formation and increase the probability of cancer when genetic changes arise instead of being the consequence of tumor progression. Understanding the methylation process in adrenal tumours could expand our knowledge of tumorigenesis and improve current therapy strategies providing potential drug targets.

Chapter 6 summarizes recent findings on epigenetics of ACC and its role in diagnosis, prognosis and therapeutic strategies.

Different studies are presented who have been able to show clustering on epigenetic level, allowing differentiation based on these specific epigenetic findings not only between benign and malignant adrenals tumors, but also correlate with ACC aggressiveness. Therefore, epigenetics is suggested a significant addition to current histological parameters. Moreover, it might serve as an addition to current ENSAT staging in order to estimate prognosis and tumor aggressiveness. In chapter 6 suggestions are made for follow up research, stimulating the implementation of a reproducible epigenetic (cluster) biomarker in the guideline. Also, a new flowchart on the potential management of the adrenal mass with the implementation of genomic analysis is presented. 
The addition of a well reproducible biomarker would be of significance, where current diagnostic and prognostic markers occasionally are unable to differentiate (borderline) malignant from benign and insufficiently correlate with biological behavior of ACC.

Chapter 6 could form the base of which in the future a study protocol could be proposed to prospectively compare the pathologic classification (Ki67 and Weiss score) of adrenal tumors versus a genomic assay versus the combination of both (PA vs genomics vs PA + genomics) in the process of accurately diagnosing adrenal tumors.

\section{Personalization}

The impact of this thesis in general, is that it attempts to individualize diagnosis, prognostication and treatment of ACC. Health professionals are often uncomfortable with prognostic uncertainty but the process of addressing "what to expect" for an individual's disease course is essential for meaningful decision-making and end-of-life planning. Additional information on prognostication as provided in this thesis could support such conversation. The same goes for tailoring of treatment. A professional should explore: does the patient strive for quantity of life or quality of life? In the process of shared decision making, patients are required to make trade-offs between the two. Some patients are willing to endure toxicities associated with treatment in order to increase their length of life, while others value quality more and are reluctant to spend their remaining years in a compromised state. This involves weighing the risks and benefits of treatment and managing the patients' expectations and concerns. In order to do so international effort needs to be made to investigate ACC patients experience with the diagnostic process and treatment of ACC and study patient reported outcomes, which are currently scarce for ACC. Furthermore, international collaborative research should continue to invest in the optimization of current- and development of new ACC treatment. 
9

. 


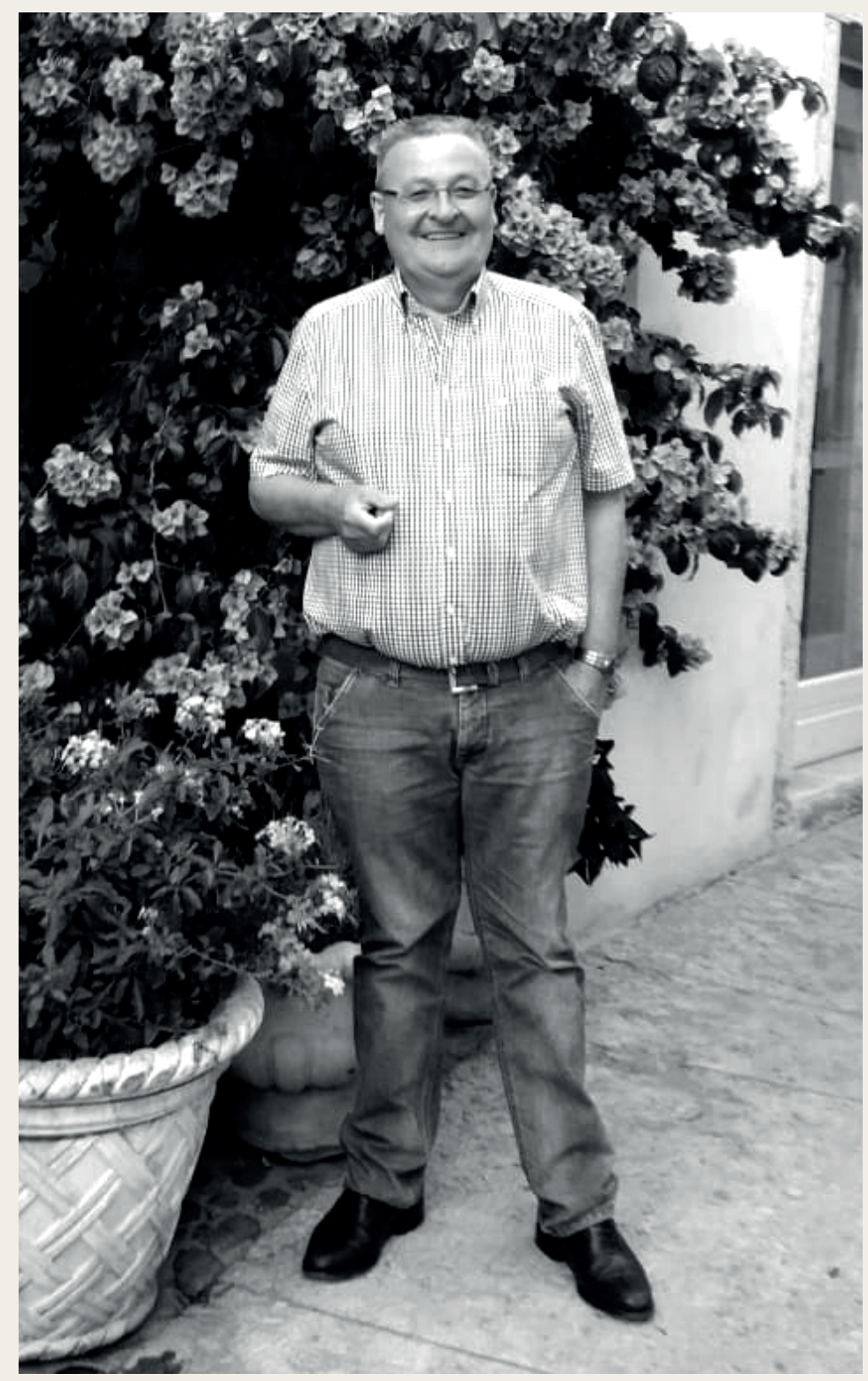


Appendix

Curriculum vitae

List of publications

Acknowledgments 


\section{Curriculum vitae}

Hester Ettaieb was born in 1988 in Naarden, the Netherlands.

After completing high school in 2006, she studied Biomedical Sciences at the University of Amsterdam. To complete this undergraduate degree, Hester undertook an internship at the NKI (Nederlands Kanker Instituut, Netherlands Cancer Institute) where she was introduced to several molecular-biological techniques used to study cancer. She obtained her Bachelor of Science in 2009 and then left Amsterdam for Maastricht where she attended the selective four-year Master's degree programme called AKO (Arts-Klinisch Onderzoeker, Doctor-Clinical Researcher) from which she graduated in 2013. In that same year she started her PhD research studying adrenocortical carcinoma under supervision of professor Harm Haak.

During her PhD she worked for over four years at the outpatient care treating patients with adrenal tumours, as ANIOS (resident not in training) on call at the Internal Medicine department and part-time as the only doctor on shift during the evenings and nights at the Maxima Medical Center Eindhoven for emergency care.

In 2018 she worked as ANIOS at the different oncology departments of the Antoni van Leeuwenhoek hospital (NKI) and in 2019 started working at Tergooi hospital at the department of Internal Medicine.

Currently she is attending residency training in Internal Medicine at the Amsterdam University Medical Center and Tergooi hospital. 


\section{List Of Publications}

Crona J, Baudin E, Terzolo M, Chrisoulidou A, Angelousi AG, Ronchi CL, Lamas C, Nieveen van Dijkum E, Ceccato F, Borson-Chazot F, Reimondo G, Tiberi GA, Ettaieb H, Kiriakopoulos A, Canu L, Kastelan D, Osher E, Yiannakopoulou E, Arnaldi G, Assie G, Paiva I, Bourdeau I, Newell-Price J, Nowak KM, Romero MT, De Martino MC, Bugalho MJ, Sherlock M, Vantyghem MC, Dennedy MC, Loli P, Rodien P, Feelders RA, de Krijger RR, Van Slycke S, Aylwin S, Morelli V, Vroonen L, Shafigullina Z, Bancos I, Trofimiuk-Muldner M, Quinkler M, Luconi M, Kroiss M, Naruse M, Igaz P, Mihai R, Della Casa S, Berruti A, Fassnacht M \& Beuschlein F 2020 ENSAT Registry-Based Randomized Clinical Trials for Adrenocortical Carcinoma. European journal of endocrinology 2020.

Bancos, I., Taylor, A.E., Chortis, V., Sitch, A.J., Jenkinson, C., Davidge-Pitts, C.J., Lang, K., Tsagarakis, S., Macech, M., Riester, A. et al. Urine Steroid Metabolomics for the Differential Diagnosis of Adrenal Incidentalomas in the EURINE-ACT Study: A Prospective Test Validation Study. Lancet Diabetes Endocrinol. 2020, 8, 773-781.

Creemers, S.G., Feelders, R.A., Valdes, N., Ronchi, C.L., Volante, M., van Hemel, B.M., Luconi, M., Ettaieb, M.H.T., Mannelli, M., Chiara, M.D. et al. The IGF2 Methylation Score for Adrenocortical Cancer: An ENSAT Validation Study. Endocr. Relat. Cancer 2020, 27, 541-550.

Ettaieb, M., Kerkhofs, T., van Engeland, M., Haak, H. Past, Present and Future of Epigenetics in Adrenocortical Carcinoma. Cancers 2020, 12, 1218.

Ettaieb, M.H.T., van Kuijk, S.M.J., de Wit-Pastoors, A., Feelders, R.A., Corssmit, E.P.M., Eekhoff, E.M.W., van der Valk, P., Timmers, H.J.L.M., Kerstens, M.N., Klumpen, H.J. et al. Development and Internal Validation of a Multivariable Prediction Model for Adrenocortical-CarcinomaSpecific Mortality. Cancers (Basel) 2020, 12, 10.3390/cancers12092720.

Yin, A., Ettaieb, M.H.T., Swen, J.J., van Deun, L., Kerkhofs, T.M.A., van der Straaten, R.J.H.M., Corssmit, E.P.M., Gelderblom, H., Kerstens, M.N., Feelders, R.A. et al. Population Pharmacokinetic and Pharmacogenetic Analysis of Mitotane in Patients with Adrenocortical Carcinoma: Towards Individualized Dosing. Clin. Pharmacokinet. 2020.

Berruti, A., Libe, R., Lagana, M., Ettaieb, H., Sukkari, M.A., Bertherat, J., Feelders, R.A., Grisanti, S., Cartry, J., Mazziotti, G. et al. Morbidity and Mortality of Bone Metastases in Advanced Adrenocortical Carcinoma: A Multicenter Retrospective Study. Eur. J. Endocrinol. 2019, 180, 311-320.

Sbiera, S., Sbiera, I., Ruggiero, C., Doghman-Bouguerra, M., Korpershoek, E., de Krijger, R.R., Ettaieb, H., Haak, H., Volante, M., Papotti, M. et al. Assessment of VAV2 Expression Refines Prognostic Prediction in Adrenocortical Carcinoma. J. Clin. Endocrinol. Metab. 2017, 102, 3491-3498. 
Ettaieb, M.H., Duker, J.C., Feelders, R.A., Corssmit, E.P., Menke-van der Houven van Oordt,C.W., Timmers, H.J., Kerstens, M.N., Wilmink, J.W., Zelissen, P.M., Havekes, B. et al. Synchronous Vs. Metachronous Metastases in Adrenocortical Carcinoma: An Analysis of the Dutch Adrenal Network. Horm. Cancer. 2016, 7, 336-344.

Kerkhofs, T.M., Derijks, L.J., Ettaieb, H., den Hartigh, J., Neef, K., Gelderblom, H., Guchelaar, H.J., Haak, H.R. Development of a Pharmacokinetic Model of Mitotane: Toward Personalized Dosing in Adrenocortical Carcinoma. Ther. Drug Monit. 2015, 37, 58-65.

Kerkhofs, T.M., Ettaieb, M.H., Hermsen, I.G., Haak, H.R. Developing Treatment for Adrenocortical Carcinoma. Endocr. Relat. Cancer 2015, 22, R325-38.

Kerkhofs, T.M., Derijks, L.J., Ettaieb, M.H., Eekhoff, E.M., Neef, C., Gelderblom, H., den Hartigh, J., Guchelaar, H.J., Haak, H.R. Short-Term Variation in Plasma Mitotane Levels Confirms the Importance of Trough Level Monitoring. Eur. J. Endocrinol. 2014, 171, 677 683.

Kerkhofs, T.M., Ettaieb, M.H., Verhoeven, R.H., Kaspers, G.J., Tissing, W.J., Loeffen, J., Van den Heuvel-Eibrink, M.M., De Krijger, R.R., Haak, H.R. Adrenocortical Carcinoma in Children: First Population-Based Clinicopathological Study with Long-Term Follow-Up. Oncol. Rep. 2014, 32, 2836-2844. 


\section{Dankwoord}

Er zijn veel momenten geweest dat ik mijn promotie aan de wilgen wilde hangen.

De enige reden om toch door te blijven zetten en te accepteren dat het niet is geworden zoals ik vooraf had beoogd, zijn de patiënten geweest. Enkelen schitteren op de kaft van mijn proefschrift als eerbetoon aan alle patiënten met bijnierschorskanker.

Tevens is het proefschrift een nagedachtenis aan Erna, Ad, Jose, Robby, Rob, Louisa, Masja en alle bijnierschorskanker patiënten die helaas aan de gevolgen hiervan zijn overleden. Het doet mij verdriet dat ik dit proefschrift niet meer met hen kan delen.

Naast de patiënten zijn er nog velen zonder wiens directe of indirecte hulp dit proefschrift niet tot stand had kunnen komen en aan wie ik daarom dank verschuldigd ben.

Allereerst prof. dr. Haak. Harm, dank voor deze unieke kans om op deze wijze te promoveren. Ik heb er heel veel van geleerd.

Dr. Eekhoff. Beste Marelise, hartelijk dank dat jij mijn copromotor wilde zijn. Graag dank ik je ook voor de samenwerking tijdens diverse onderdelen van dit promotie-traject.

Dan de leden van de beoordelingscommissie, prof. dr. Tjan-Heijnen, prof. dr. Bouvy, prof. dr. de Bruïne, dr. Dahele en prof. dr. de Herder. Graag bedank ik u voor de tijd en moeite die $\mathrm{u}$ in het grondig lezen en beoordelen van mijn proefschrift hebt gestoken.

Verder wil ik alle medeauteurs bedanken die in variërende frequentie en intensiteit hebben meegewerkt aan de artikelen waaruit dit proefschrift is opgebouwd.

I would like to especially thank Anyue Yin: I really enjoyed working with you on the pharmacokinetics paper and I am very grateful that you were available to discuss this work with me during several evenings.

Tevens wil ik graag Sander van Kuijk in het bijzonder bedanken voor het delen van zijn kennis over predictiemodellen en voor zijn geduld in de totstandkoming van hoofdstuk 3 van dit proefschrift.

Graag bedank ik alle specialisten van het Bijnier Netwerk Nederland voor de prettige samenwerking tijdens de afgelopen jaren.

Alle semi-artsen die tijdens hun wetenschapsstages hebben bijgedragen aan de database en artikelen: Annelies Pastoors, Janneke Duker en Liselotte van Deun, inmiddels alle drie praktiserend huisarts!

De secretaresses interne geneeskunde/oncologie en in het bijzonder Claudia Janssen en Suzanne van Mook bedank ik voor de fijne samenwerking en jullie hulp bij alle logistiek van de bijnierpatiënten. 
Anita Groos, als analiste op het laboratorium betrokken bij de bijnierstudies. Veel dank voor jouw zorgvuldigheid en accuraatheid bij het beheren van de opslag van het patiëntmateriaal.

Mijn collegae arts-onderzoekers Dorien Lobbezoo, Roosmarijn Lemmers, Steffie Brouns, Anne Maas, Tonneke Beijers, Thomas Kerkhofs en Sunny Singh, die veel positiviteit hebben gebracht tijdens mijn tijd als arts-onderzoeker en escalatie-arts in het Maxima Medisch Centrum. In het bijzonder wil ik Joyce Wachelder en Hao Ran Cheng bedanken, uit ons samenwerken is een vriendschap gegroeid: Yesterday is History, Today is a Gift, Tomorrow is Mystery.

Rebecca Steenaard wil ik bedanken voor de vlekkeloze overname van het bijnieronderzoek en de patiëntenzorg.

Het Escalatieteam wil ik bedanken voor de belangstelling en de interessante en fijne humoristische gesprekken tijdens de diensten in Eindhoven.

Mijn collegae (specialisten en arts-assistenten) van de vakgroep interne geneeskunde/ maag-darmleverziekten van het Maxima Medisch Centrum voor de fijne samenwerking en de geslaagde niet werk gerelateerde activiteiten.

Ook mijn collegae van het Antoni van Leeuwenhoek ziekenhuis wil ik bedanken voor een heel mooie tijd: in het bijzonder bedank ik Matthias Karger. Wat heb ik ontzettend veel van jou geleerd. Niet alleen wat betreft de geneeskunde maar ook over filosofie.

Mijn collegae in het Tergooi ziekenhuis, door wie ik met plezier naar mijn werk ga. In het bijzonder veel dank aan Marjolein Rentinck, een geweldige opleidster interne geneeskunde. Dank je wel voor jouw vertrouwen en steun.

\section{Vrienden}

Lieve vrienden, dank voor jullie steun, goede raad en inspiratie.

Ze zeggen: gedeelde smart is halve smart. Nicole Verheuvel, samen zijn wij the underdogs'. Wat heerlijk om altijd bij jou te mogen ventileren onder het genot van een high wine, tijdens een massage of een Disneyfilm. Ik hoop deze therapie nog lang te mogen voortzetten. Jij begrijpt mij.

Alex van der Wateren, we leerden elkaar kennen tijdens de studie biomedische wetenschappen. Hoewel op afstand, ben je mijn beste vriendin. Je bent me ontzettend dierbaar. Je hebt me de afgelopen jaren enorm gesteund met je lieve woorden, voice messages, appjes, e-mails en cadeaux (staat er opeens een bos bloemen op mijn werk, etc.). Tevens heb je me vaak geholpen met de Engelse taal en grammatica. Je bent een heel mooi mens. Ik blijf stiekem hopen dat je weer terugkomt naar Nederland. 


\section{Mijn familie}

Ineke Vonk, dank dat je altijd interesse bleef tonen en probeerde te begrijpen wat ik nou toch allemaal precies aan het doen was.

Eveline de Groot, jij ook bedankt voor jouw interesse en het kunnen delen van frustraties (jij met de PABO-opleiding, ik met mijn promotie).

Piet de Groot and Ann May thank you for all the lovely dinners and glasses of red wine. Ann, your (vegan) food has been comforting during tough PhD times. Thank you for proofreading the discussion of my thesis. Piet het beloofde mooie schilderij aan de muur is altijd een goede motivatie geweest om dit proefschrift te voltooien.

Jonah en familie Knetemann, dank jullie wel voor de interesse in mijn bezigheden en alle gezellige gelegenheden tijdens de afgelopen jaren.

Charlotte, mijn mooie lieve zus en vriendin. Je hebt mij o.a. leren lezen, zwemmen en auto rijden. Ik kon altijd alles afkijken van mijn slimme, hardwerkende zus. Wellicht dat dit ook de reden is dat deze promotie zo lang heeft geduurd. Ik kon het niet bij jou afkijken.

Eva, wat ben je toch een heerlijk kind. Ik geniet ervan jouw tante te zijn. Alors on danse!

Tonny en Ben, mamma en pappa. Mijn dank aan jullie is niet in enkele woorden op papier te zetten. Onvoorwaardelijke liefde en steun is wat jullie mij altijd hebben gegeven. Jullie hebben altijd in mij geloofd en mij in alles gefaciliteerd. Jullie hebben mij geleerd hard te werken en mijn eigen geluk te creëren. "Als je jezelf niet kietelt, lach je nooit", zei oma Teunisje Hendrika Moos. Zonder jullie inzet en investeringen had ik niet zo'n mooi leven gehad. Dit proefschrift draag ik daarom aan jullie op. Mille mercis.

Lieve Gabe, woorden schieten me tekort. Na vier jaar lang (trein)reizen tussen Amsterdam en Maastricht, bezoeken aan al mijn coschap locaties, heb je zelfs je huis in Amsterdam verkocht om te gaan samenwonen in Brabant. Dit alles deed je zodat ik kon promoveren en mijn dromen kon najagen. Je hebt jezelf vaak weggecijferd zonder hierover te klagen. Ik weet niet hoe ik je ooit kan bedanken. Ik had dit never nooit zonder jou gekund. Tijdens mijn promotie heb ik vol bewondering je zien afstuderen tot meester in de rechten naast je full time baan. Wat ben ik trots op je. 'Teamwork makes the dream work.' Op naar nog veel mooie avonturen 


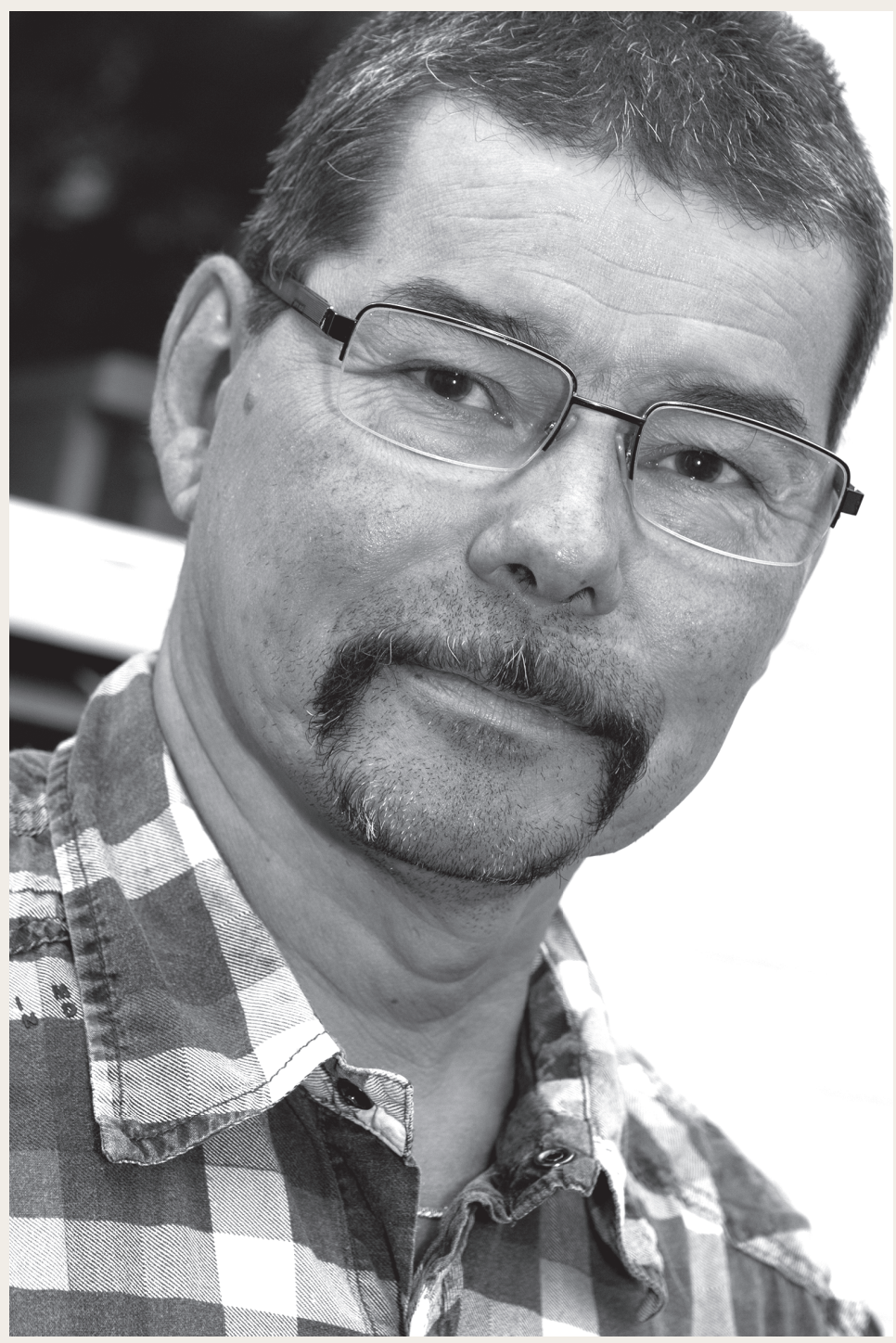


A 


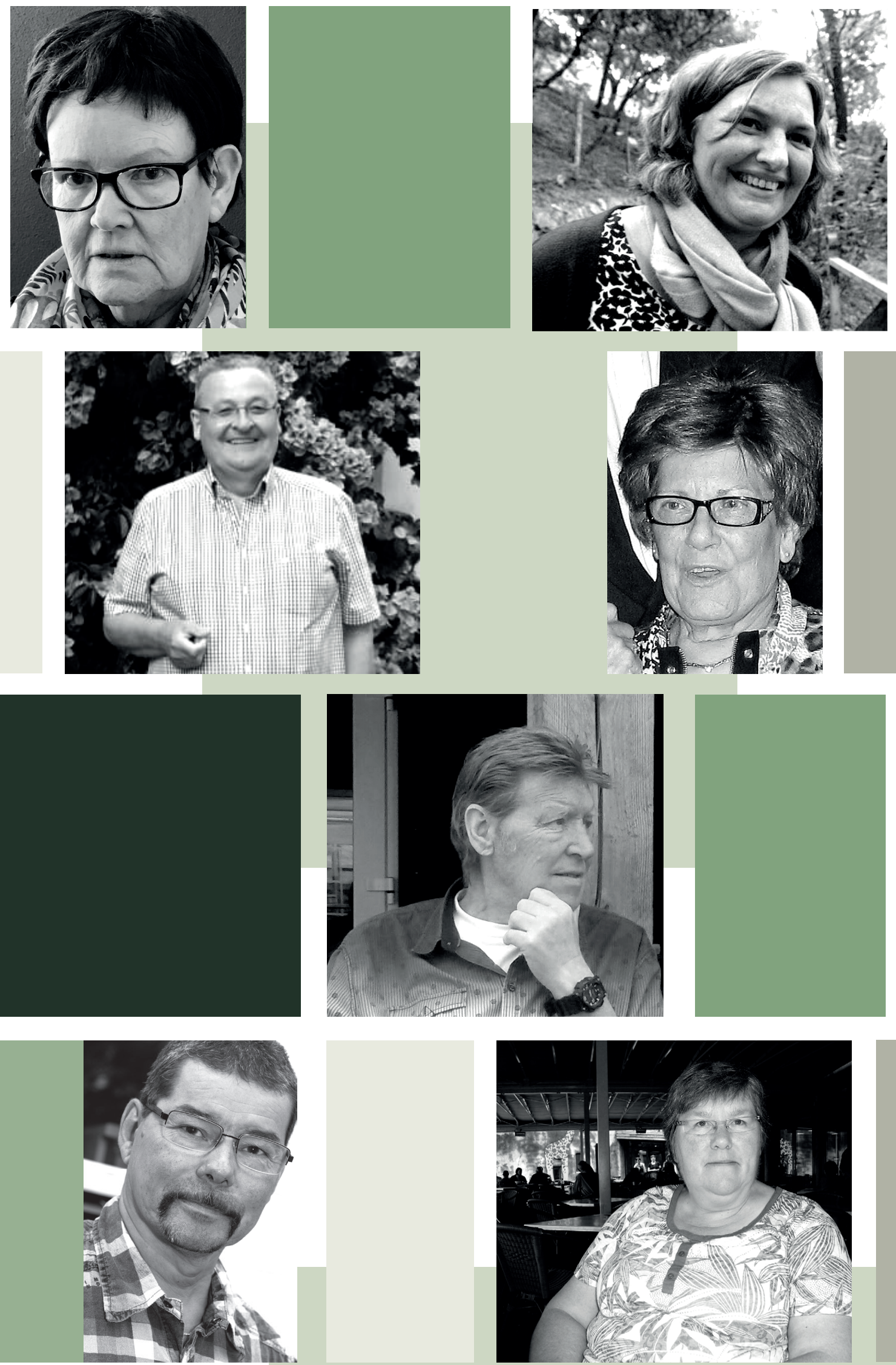